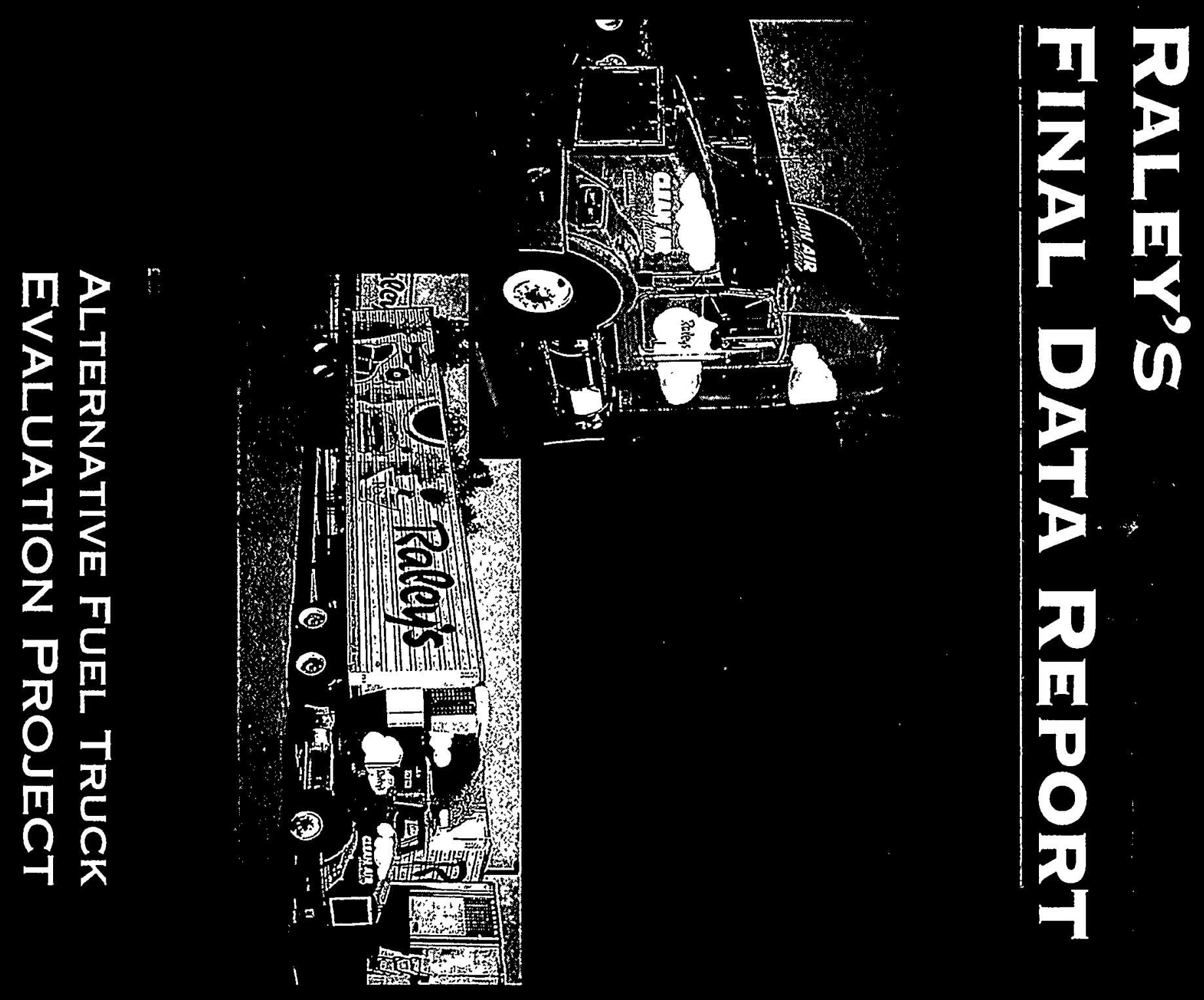




\title{
Raley's LNG Truck Site Final Data Report
}

DOE/NREL Alternative Fuel Truck Evaluation Project

\author{
For \\ National Renewable Energy Laboratory \\ 1617 Cole Boulevard \\ Golden, Colorado 80401
}

July 1999

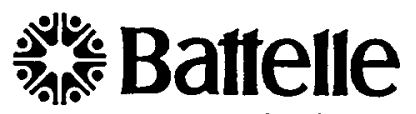

... Putting Technology To Work

505 King Avenue

Columbus, Ohio 43201-2693 


\section{DISCLAIMER}

This report is a work prepared for the United States Government by Battelle. In no event shall either the United State Government or Battelle have any responsibility or liability for any consequences of any use, misuse, inability to use, or reliance upon the information contained herein, nor does it either warrant or otherwise represent in any way the accuracy, adequacy, efficacy, or applicability of the contents hereof. 


\section{DISCLAIMER}

This report was prepared as an account of work sponsored by an agency of the United States Government. Neither the United States Government nor any agency thereof, nor any of their employees, make any warranty, express or implied, or assumes any legal liability or responsibility for the accuracy, completeness, or usefulness of any information, apparatus, product, or process disclosed, or represents that its use would not infringe privately owned rights. Reference herein to any specific commercial product, process, or service by trade name, trademark, manufacturer, or otherwise does not necessarily constitute or imply its endorsement, recommendation, or favoring by the United States Government or any agency thereof. The views and opinions of authors expressed herein do not necessarily state or reflect those of the United States Government or any agency thereof. 


\section{DISCLAIMER}

Portions of this document may be illegible in electronic image products. Images are produced from the best available original document. 


\section{Table of Contents}

Page

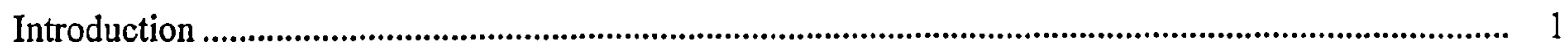

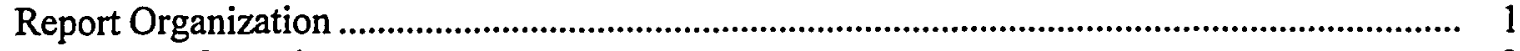

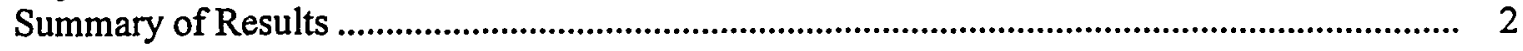

Overview of Evaluation Site and Data Presentation ...................................................................... 4

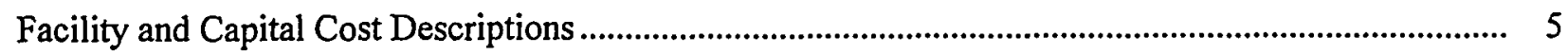

Vehicle System and Expected Operation Descriptions .............................................................. 9

Vehicle System Descriptions.................................................................................................. 9

Duty Cycle Descriptions ..................................................................................................... 10

Operational Results: Fuel Consumption, Engine Oil Consumption, and Maintenance Costs.................. 14

Data Collection Methods........................................................................................................... 14

Summary of Operational Results...................................................................................... 15

Fuel Consumption, Fuel Economy, and Cost ................................................................... 15

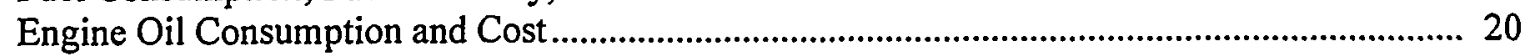

Maintenance, Maintenance Costs, and Warranty ....................................................................... 21

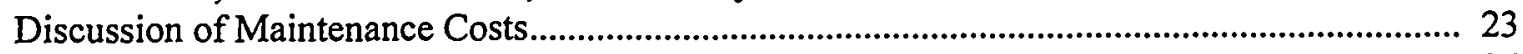

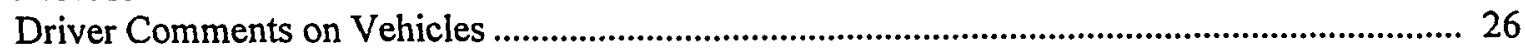

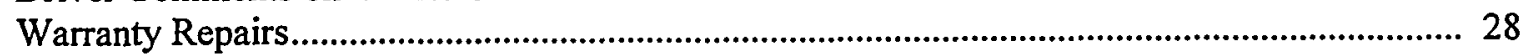

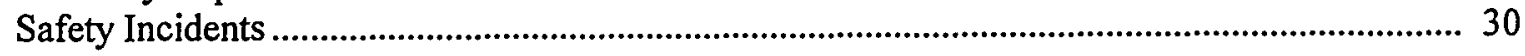

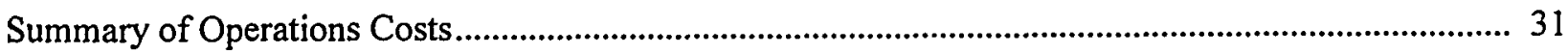

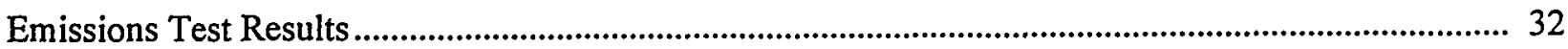

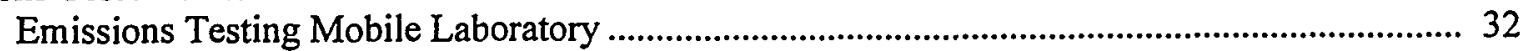

Emissions Test Results at Raley's ..................................................................................... 32

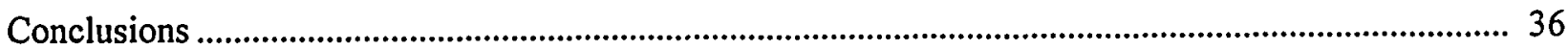

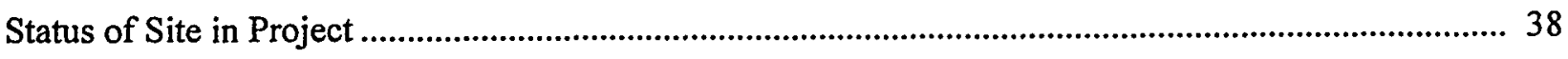

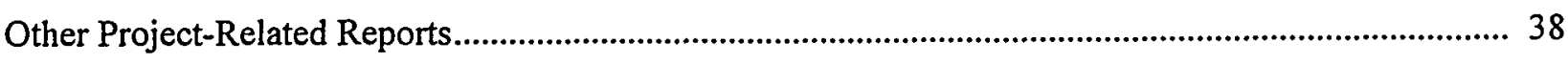

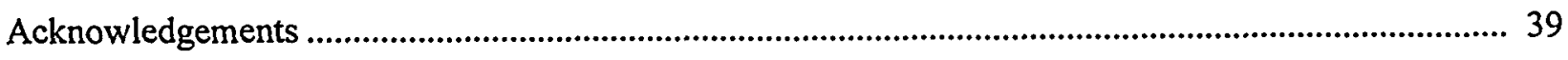

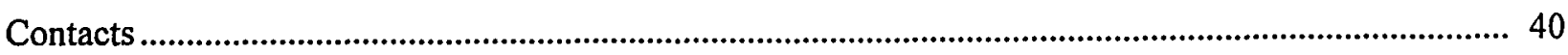




\section{List of Tables}

Table 1. Vehicle System Descriptions - Raley's.

Table 2. Truck Usage Per Trip and Per Day Before Clean Point (May 97 - December 97)................. 11

Table 3. Truck Usage Per Trip and Per Day After Clean Point (January 98 - December 98) ................ 11

Table 4. Average Monthly Mileage..................................................................................................... 12

Table 5. Fuel Consumption and Economy Before Clean Point (April 97 - December 97) .................. 17

Table 6. Fuel Consumption and Economy After Clean Point (January 98 - December 98).................. 17

Table 7. Monthly Average Fuel Costs....................................................................................................... 19

Table 8. Fuel Usage Cost Analysis..................................................................................................... 20

Table 9. Maintenance Costs............................................................................................................. 22

Table 10. Maintenance Costs by Vehicle System ................................................................................ 24

Table 11. Number of Driver Complaints by Category ……………………………………………….... 27

Table 12. Cummins Warranty Engine Items Replaced by Truck ............................................................ 29

Table 13. Summary of Operations Costs .............................................................................................. 31

Table 14. Summary of Emissions Testing Results ............................................................................. 33

Table 15. Heavy Engine Emissions Certification Standards and

Certification Data for Raley's Trucks (grams per brake-horsepower hour)..................... 35

\section{List of Figures}

Figure 1. LNG Refueling at the Quick Response System (QRS) Temporary Station.......................... 5

Figure 2. Raley's Distribution Center ..................................................................................................... 6

Figure 3. Raley's Permanent LNG Refueling Station............................................................................... 7

Figure 4. LNG Trucks Parked Outside at the Distribution Center ............................................................ 7

Figure 5. Maintenance Facility For All Raley's Trucks at Ozark Trucking........................................... 7

Figure 6. LNG Yard Tractor at Work................................................................................................. 8

Figure 7. Average Monthly Mileage Per Truck ...................................................................................... 12

Figure 8. LNG Truck at Raley's Being Fueled .......................................................................................... 16

Figure 9. Number of Driver Complaints for Low Power and

Rough Operation by Month for LNG Trucks................................................................... 28

Figure 10. Number of Cummins Warranty Claims by Month for LNG Trucks...................................... 29

Figure 11. WVU Five-Peak Truck Cycle ................................................................................................ 33

Figure 12. WVU Dynamometer Laboratory at Raley's ............................................................................ 34

\section{List of Appendices}

Appendix A. Map of Raley's Locations and Listing of Locations

Appendix B. Vehicle Descriptions

Appendix C. Detail Trip Summary, Average Speed Summary, and Average Monthly Mileage Summary

Appendix D. Summary Statistics

Appendix E. Monthly Fuel and Engine Oil Consumption by Truck and Fuel Economy Analysis

Appendix F. LNG and Diesel Fuel Costs

Appendix G. Monthly Maintenance Costs by Truck and Driver Complaint Summary

Appendix H. Emissions Testing Results 


\section{Final Data Report \\ Raley's LNG Truck Site}

\section{Introduction}

Raley's is a 120-store grocery chain with headquarters in Sacramento, California, that has been operating eight heavy-duty LNG trucks (Kenworth T800 trucks with Cummins L10-300G engines) and two LNG yard tractors (Ottawa trucks with Cummins B5.9G engines) since April 1997. This report describes the results of data collection and evaluation of the eight heavy-duty LNG trucks compared to similar heavyduty diesel trucks operating at Raley's. The data collection and evaluation are a part of the U.S. Department of Energy (DOE)/National Renewable Energy Laboratory (NREL) Alternative Fuel Truck Evaluation Project. Several trucking companies are planned to participate in the evaluation project. To date, four truck sites have been added to the project, including:

- Raley's (Sacramento, California)

- Pima Gro Systems, Inc./Orange County Sanitation District (Fountain Valley, California)

- Waste Management (Washington, Pennsylvania)

- United Parcel Service (Hartford, Connecticut)

The sites in this project have been selected by NREL and Battelle (NREL's subcontractor for this project) according to the alternative fuel technology, type of trucks and engines, availability of diesel comparison ("control") vehicles, and interest from the trucking company in participation. The intent for this project is to select sites operating the newest alternative fuel technology available for medium- and heavy-duty trucks.

Comparative data are being collected on operations, maintenance, fueling, performance, and emissions characteristics of each alternative fuel fleet and comparable diesel control fleet operating at the same site. The data collection has been designed to cause as little disruption for the host sites as possible. In most cases, staff at the participating site send copies (electronic and/or paper) of data already part of normal business operations. All fleets participating in the project have access to all data being collected from their site and other data available from the project. Summaries of the data collected, evaluations, and analyses of the data are distributed to designated staff at the host site for review and input.

\section{Report Organization}

This report includes a technical review of data collected from the Raley's LNG truck site. Raley's was chosen because of their dedication to integrating the LNG trucks into their daily operations. This report is 
intended to serve as a summary of data collection, analysis, and evaluation. The report is organized into six chapters:

- Overview of Evaluation Site and Data Presentation

- Facility and Capital Cost Descriptions

- Vehicle System and Expected Operation Descriptions

- Operational Results: Fuel Consumption, Engine Oil Consumption, and Maintenance Costs

- Summary of Operating Costs

- Emissions Test Results.

\section{Summary of Results}

The major conclusions from the evaluation of the Raley's LNG truck site include the following:

- The LNG trucks have been used as planned. The LNG trucks have been used for local Sacramento area pickup and delivery at the request of the Sacramento Metropolitan Air Quality Management District in return for funding to help establish LNG operations.

- Drivers perceive the LNG trucks to be slightly under-powered for Raley's operation.

- The emissions results from the Raley's project show large decreases in NOx and PM for the LNG trucks. The NOx reduction for the eight LNG trucks using the West Virginia University (WVU) testing results would have a nearly 7 tons per year reduction compared to diesel trucks running in place of the LNG trucks.

- Energy equivalent fuel economies between in-use fuel data and emissions testing fuel economies are essentially consistent. The energy equivalent fuel economy comparison between LNG and diesel trucks at Raley's is about 38 percent lower for the LNG trucks. This has caused some problems with range for the LNG trucks. This lower fuel economy has been aggravated by having fuel gauges that are not reliable.

- Mileage per vehicle per calendar month and year was significantly lower for the LNG trucks compared to the diesel control trucks. This lower mileage for the LNG trucks was caused by how the trucks have been used (routes closer to the Distribution Center in the Sacramento area) as well as problems with lower range than the diesel trucks and engine problems experienced early in the data collection period. The lower vehicle mileage for the LNG trucks has caused the cost per mile for maintenance to be biased higher than expected because the preventive maintenance actions are performed on a quarterly basis, not a mileage basis. To better understand how the LNG trucks have performed, the maintenance cost analysis has been made on a cost per truck basis for the same length of data period to account for this bias. Maintenance costs per mile are also provided. 
- Operations and LNG fuel costs during the evaluation were affected by a change from a temporary to a permanent LNG fueling and storage station at Raley's. There were several major delays in starting and completing the construction of the permanent LNG station. The permanent LNG station allows Raley's to purchase a full tanker truckload of LNG, thus reducing the cost by $\$ 0.10$ per LNG gallon.

- Availability of LNG in the local Sacramento, California area could reduce LNG fuel cost significantly by reducing transportation costs (currently coming from Wyoming or Arizona).

- Raley's staff indicated that their operation would benefit from a larger LNG storage tank at the permanent fueling station (currently 13,000 gallons). The tank has reserve for vapor and liquid that cannot be used, which reduces the useable volume to about 11,500 gallons. This leaves a margin for error of 1,500 gallons when ordering fuel. Fuel shipments must be scheduled carefully, or the station with either run out of fuel or will not be able to accommodate the full incoming shipment, which results in lost fuel that Raley's must pay for.

- There were some problems with the LNG engines, such as with the wastegate, ignition modules, and sensor calibration. The LNG engine manufacturer, Cummins, was supportive and responsive to these problems. Operations were fairly trouble-free at the end of the evaluation.

- Raley's intends to expand their LNG truck operations; however, there are few choices of heavyduty natural gas truck technologies.

- As part of this project, Raley's purchased two LNG yard tractors. These vehicles have been extremely successful for Raley's. The drivers have noted that, compared to the older diesel yard tractors, the LNG yard tractors offer reduced noise and vibration as well as a lack of diesel fumes. 


\section{Overview of Evaluation Site and Data Presentation}

Raley's is a 120-store grocery chain centered in Sacramento, California, with operations in the Sacramento and San Jose, California, areas as well as Reno, Nevada (a listing of store locations and maps are shown in Appendix A). At the time of this evaluation, Raley's had a 64-truck fleet with eight model year 1997, Class 8 Kenworth T800 tractors using Cummins L10-300G engines and operating on LNG fuel. The diesel control vehicles used for this study were three model year 1996, Class 8 Kenworth T400 tractors using Cummins M11-330 engines. The LNG trucks were used to haul perishable goods from the Raley's Distribution Center in Sacramento to local and nearby Raley's, Bel Air, and Nob Hill grocery locations. The diesel control trucks are used to service Raley's, Bel Air, and Nob Hill grocery locations South and West of Sacramento on routes over terrain similar to those of routes covered by the LNG trucks. The LNG trucks started operation on April 17, 1997, and the diesel vehicles started operation in late 1995 and early 1996. Raley's also has two Ottawa LNG yard tractors using Cummins B5.9G engines.

The data analysis and evaluation results for vehicle and fuel usage are shown for two time periods: before January 1, 1998 (April 1997 through December 1997) and after January 1, 1998 (January 1998 through December 1998). January 1, 1998, is considered the "clean point" for the evaluation, because by that time, early problems with the natural gas engines had been resolved through engine modifications (e.g., component and engine map modifications). Most of the analyses in this report are presented as either "before clean point" or "after clean point."

The focus of this report will be on the results of operations after the clean point with a few comparisons of changes between the two data periods. Maintenance data has been compared between similar vehicle/ engine life (based on mileage and calendar time from start of operation of each truck). Maintenance data from the LNG trucks start after the data clean point. Data for the diesel control trucks match the same vehicle life as the LNG trucks. 


\section{Facility and Capital Cost Descriptions}

Prior to November 1998, refueling for the LNG trucks was provided by a 5,000-gallon Quick Response System (QRS) temporary station from MVE, Inc. (Figure 1). The temporary station was located at the Raley's Distribution Center, shown in Figure 2. A permanent 13,000-gallon LNG refueling station (Figure 3) was constructed at the Distribution Center and is estimated to cost about $\$ 350,000$. With the completion of the permanent refueling station, Raley's was able to receive a complete tanker truckload of LNG fuel (10,000 gallons) instead of a partial load (5,000 gallons). The full loads of LNG fuel cost $\$ 0.10$ per gallon less (reduced from $\$ 0.575$ to $\$ 0.475$ per LNG gallon) then the partial loads. The permanent station significantly reduced fuel costs.

At the Distribution Center, the trucks are stored outside (Figure 4). At the maintenance facility (Figure 5), the LNG trucks have the fuel system shut off before entering for maintenance. The LNG trucks are stored outside if the truck is left at the maintenance facility for more than a work shift. No changes were made to the maintenance facility and therefore there was no increased cost for the operation and servicing of LNG trucks.

Raley's leased all of the trucks taking part in the evaluation (both diesel and LNG) from Ozark Trucking. The original purchase prices were $\$ 72,000$ for the diesel trucks and $\$ 107,000$ for the LNG trucks (a difference of $\$ 35,000$ ). One of the LNG yard tractors is shown in Figure 6.

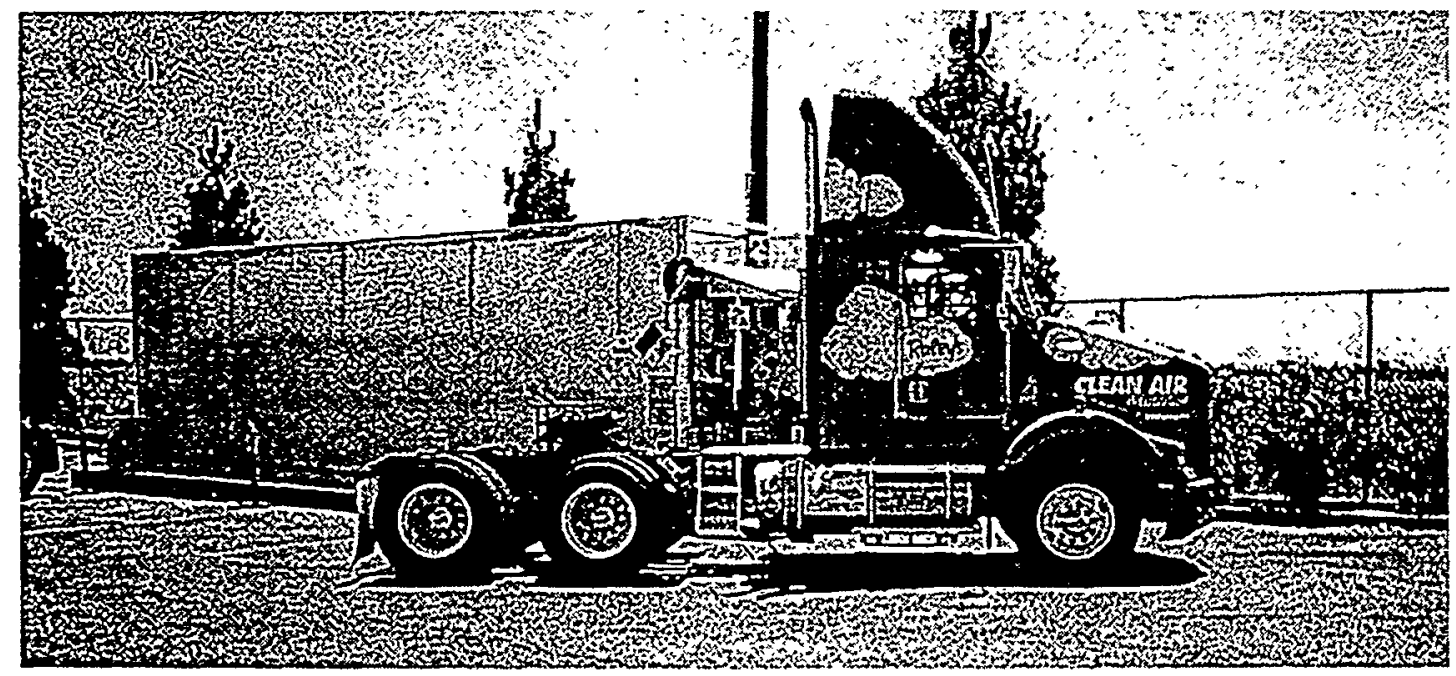

Figure 1. LNG Refueling at the Quick Response System (QRS) Temporary Station 

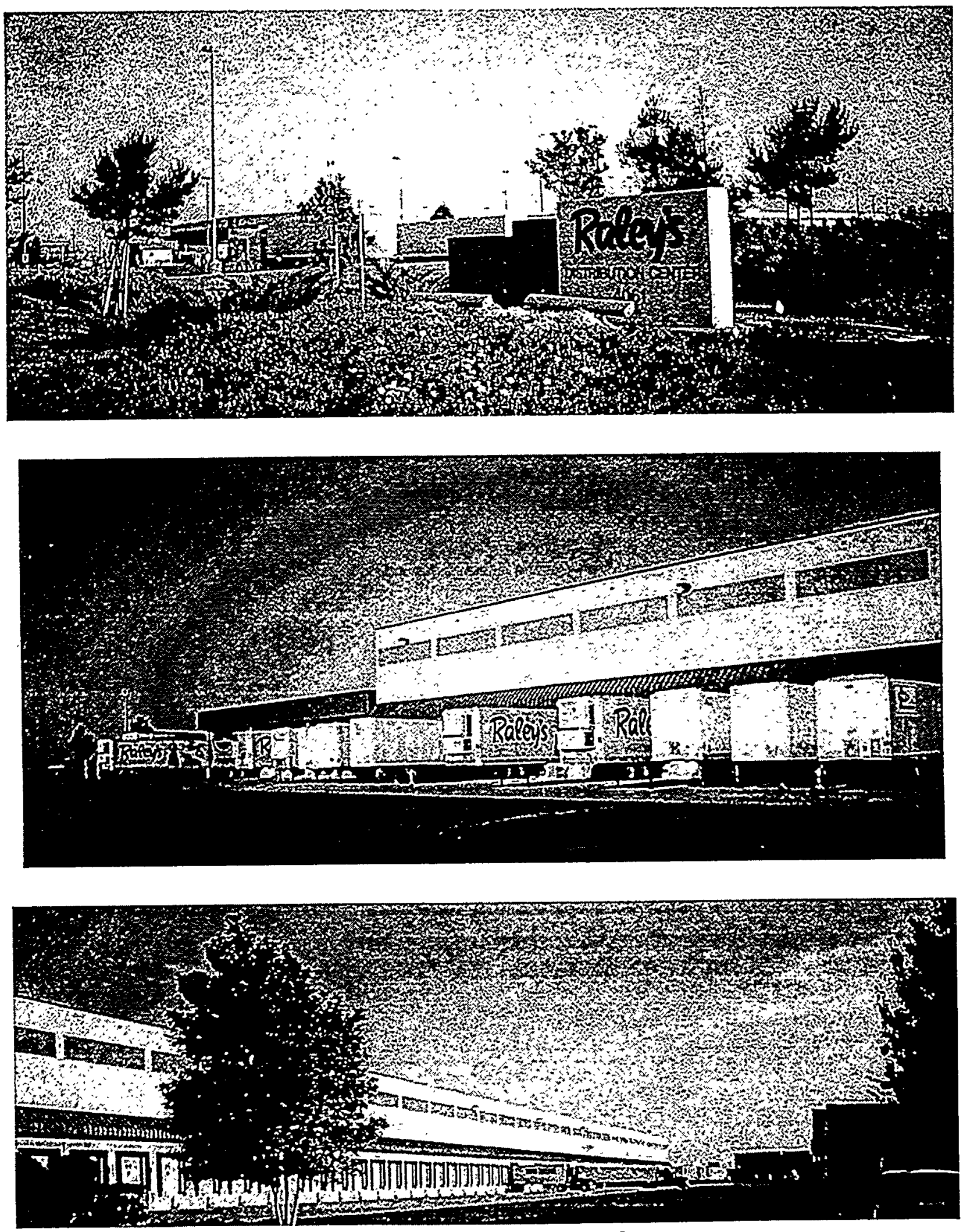

Figure 2. Raley's Distribution Center 


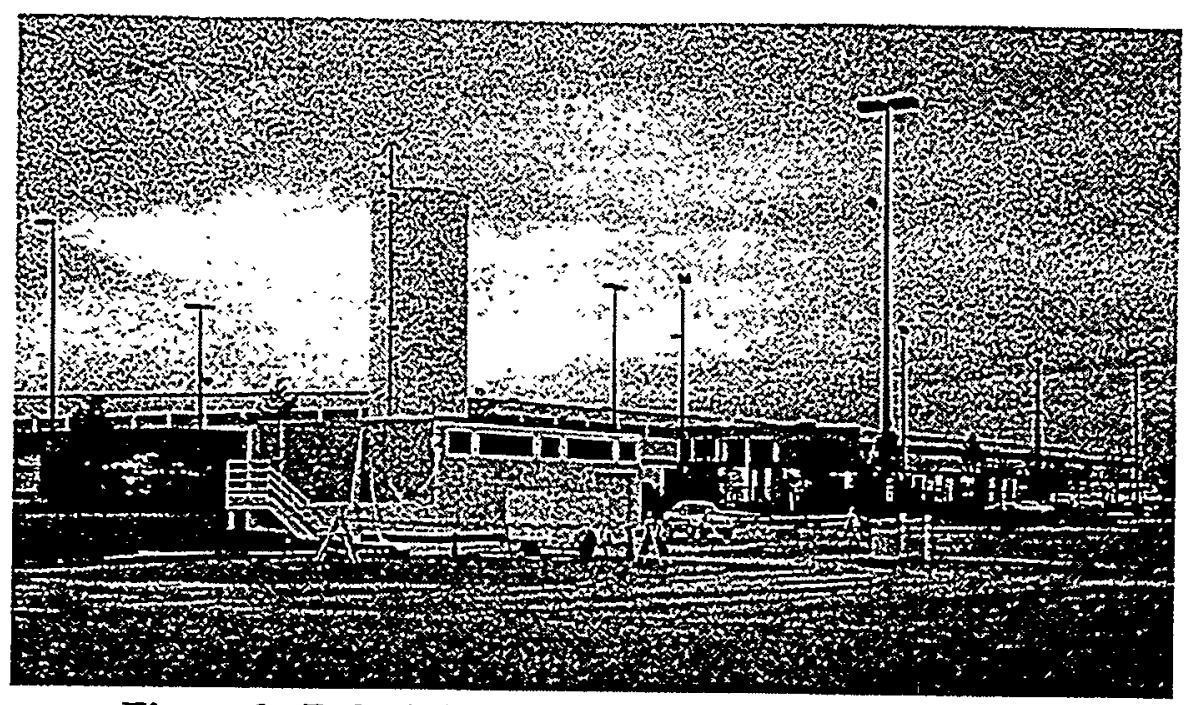

Figure 3. Raley's Permanent LNG Refueling Station

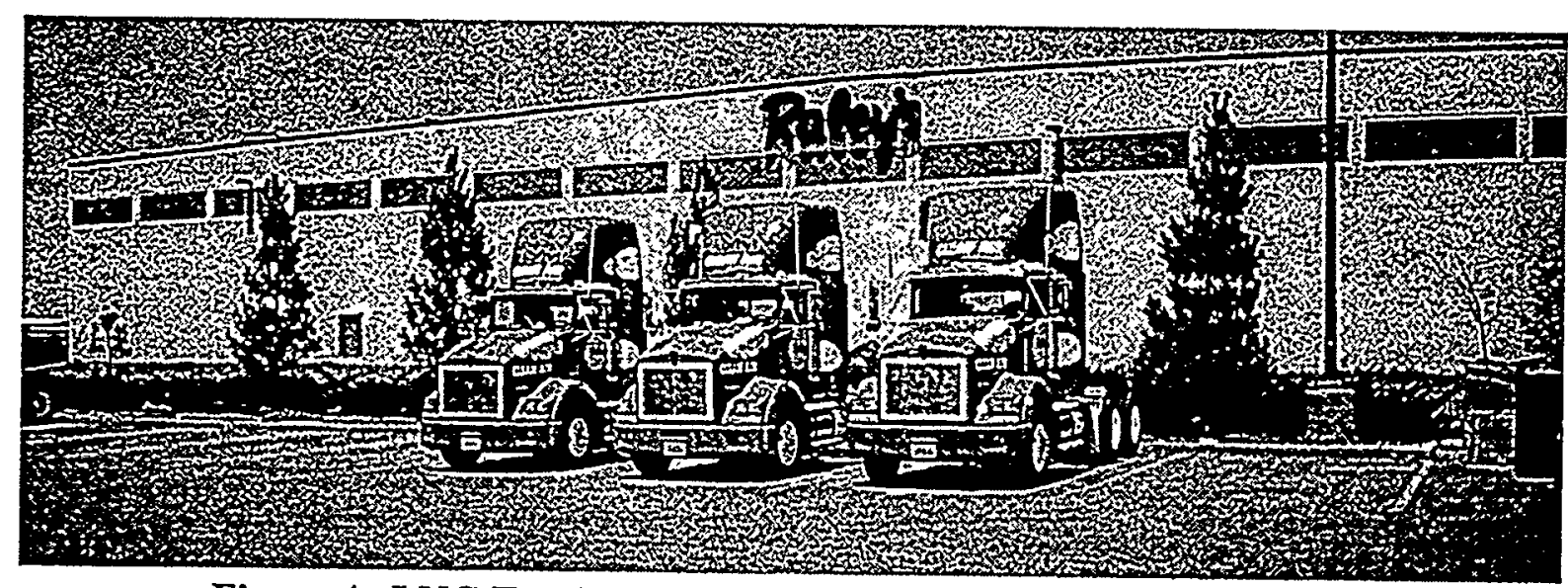

Figure 4. LNG Trucks Parked Outside at the Distribution Center

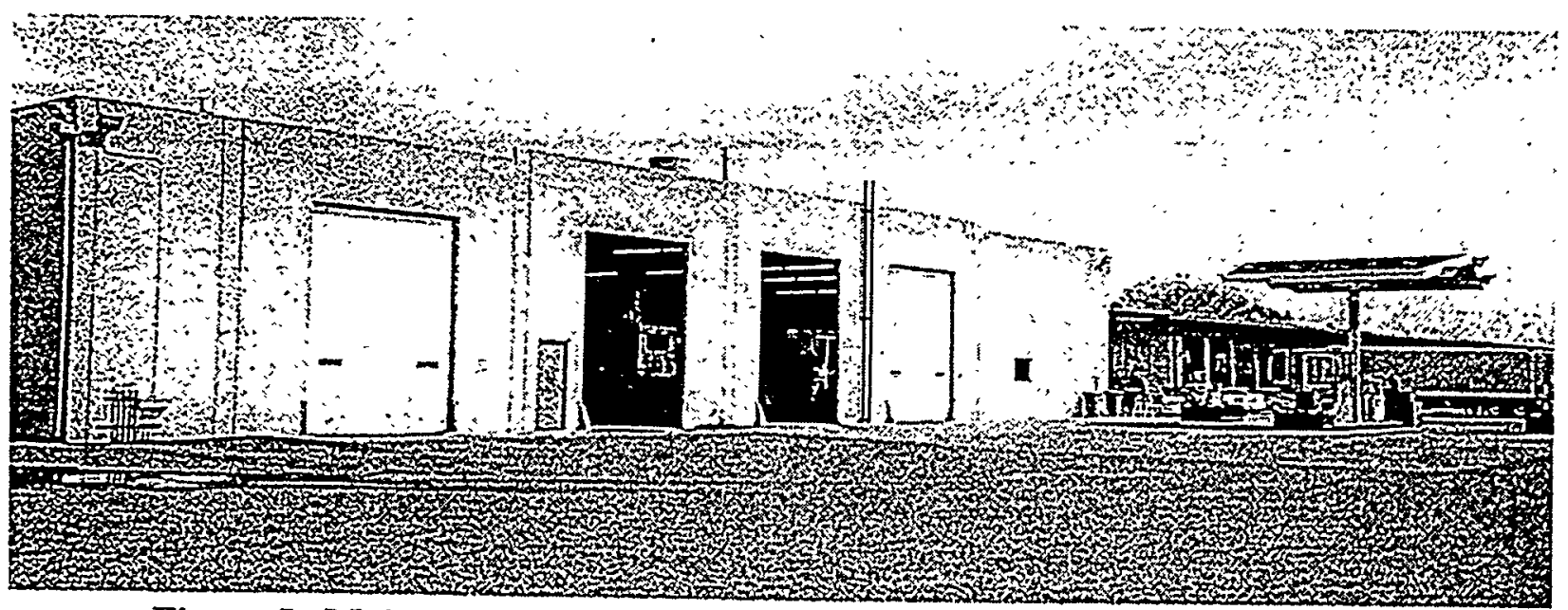

Figure 5. Maintenance Facility For All Raley's Trucks at Ozark Trucking 


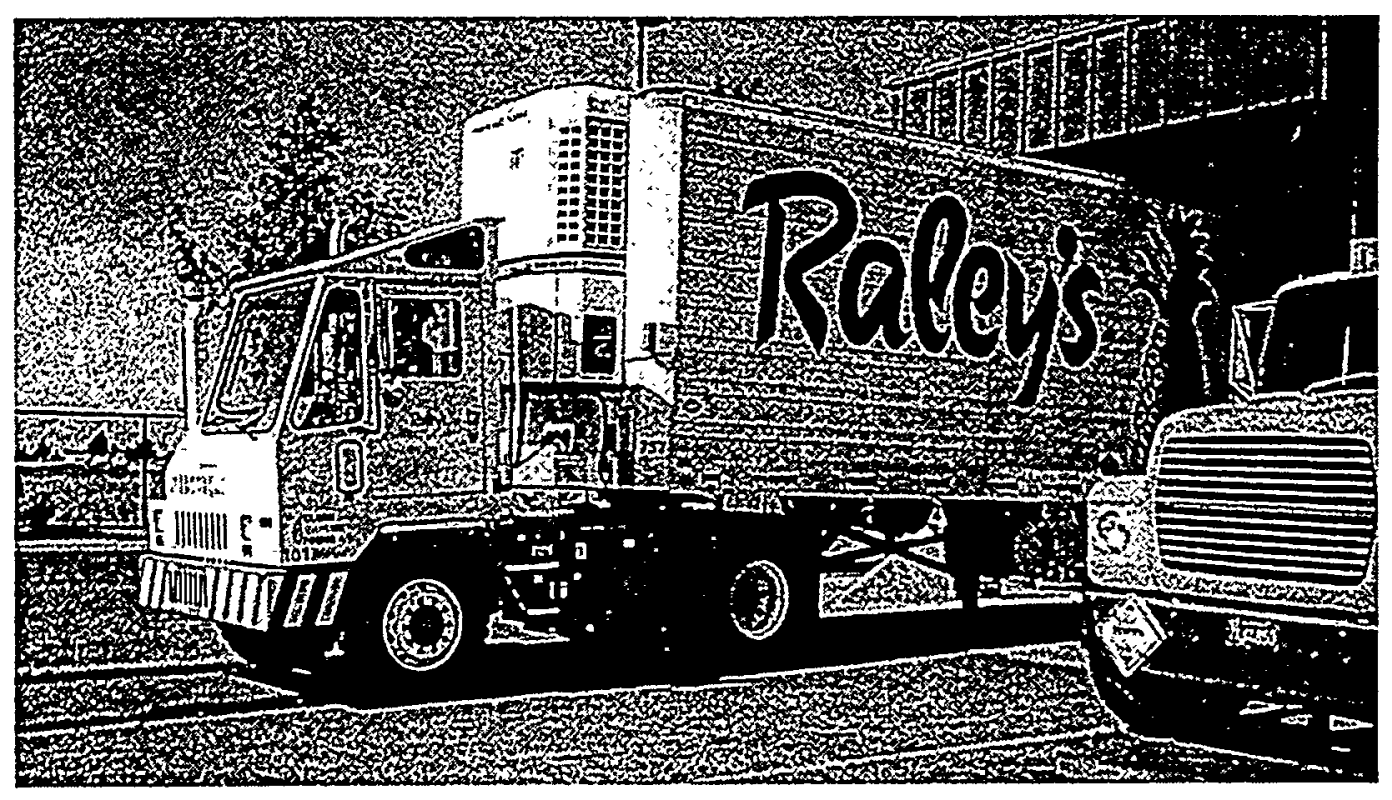

Figure 6. LNG Yard Tractor at Work 


\section{Vehicle System and Expected Operation Descriptions}

Descriptions and specifications of major systems for each vehicle in the evaluation were written at the beginning of the data collection, and were revised when any major systems were altered. The specifications describe the main systems in the vehicle powertrain as well as accessory equipment. This information documents the similarity of the LNG and diesel trucks, and also documents specific equipment that may affect the performance of the vehicles in regard to fuel economy and overall reliability.

A description of the expected vehicle operations was developed to give analysts an understanding of the amount of work the study trucks were expected to accomplish in a given time frame. The description includes the number of stores the trucks usually service in a trip or on each day, the load that the truck sees (full or partial load on delivery and/or on return), and the expected number of hours and miles in service.

\section{Vehicle System Descriptions}

The Raley's LNG and diesel truck systems are described in summary in Table 1 and in full in Appendix B. The chassis used for the trucks was the T400 for the diesel control vehicles and the T800 for the LNG vehicles. The differences between the T400 and T800 models were described as minor by a Kenworth engineer. A comparison of similar operations of these two models was considered acceptable by the Kenworth engineer. The differences between the T400 and T800 are basically that the T400 is 9 inches shorter in length (from front bumper to back of the cab) than the T800, and the T800 is slightly more aerodynamic because of its curved windshield. The standard (at Kenworth) engine and transmission for both models are the Cummins M11-330 engine and the Fuller RTX 13710C transmission. The base model price for the T400 is approximately $\$ 1,800$ cheaper than the T800. The T400 diesel control trucks at Raley's are a year older than the T800 LNG vehicles. Back maintenance data were collected for the $\mathrm{T} 400 \mathrm{~s}$, so that the maintenance cost comparisons would match trucks of similar age.

The Cummins L10-300G (LNG) and M11-330 (diesel) engines were considered to be comparable. The L10 has been phased out by Cummins for diesel truck operations. Also, Mr. Don Welliver (Cummins Chief Engineer - L10/M1 1 Natural Gas Engine Development) supported the comparison of the M11 and Cummins L10-280/300G engines for emissions testing. Mr. Welliver commented that the two engines were slightly different in configuration; however, the comparison made sense because Cummins does not have a natural gas version of the M11 engine. 
Table 1. Vehicle System Descriptions - Raley's

\begin{tabular}{|l|l|l|}
\hline \multicolumn{1}{|c|}{ Description } & \multicolumn{1}{c|}{ Diesel Control Trucks } & \multicolumn{1}{c|}{ LNG Trucks } \\
\hline Chassis Manufacturer/Model & Kenworth T400, Class 8 & Kenworth T800, Class 8 \\
\hline Chassis Model Year & 1996 & 1997 \\
\hline Engine Manufacturer/Model & Cummins M11-330 & Cummins L10-300G \\
\hline $\begin{array}{l}\text { Engine Ratings } \\
\text { Max. Horsepower } \\
\text { Max. Torque }\end{array}$ & $\begin{array}{l}330 \mathrm{hp} @ 1600 \mathrm{rpm} \\
1250 \mathrm{lb}-\mathrm{ft} @ 1200 \mathrm{rpm}\end{array}$ & $\begin{array}{l}300 \mathrm{hp} \mathrm{@} \mathrm{2100} \mathrm{rpm} \\
900 \mathrm{lb}-\mathrm{ft} \text { @ 1300 rpm }\end{array}$ \\
\hline Fuel System Storage Capacity & 114 gallons & $\begin{array}{l}174 \mathrm{LNG} \text { gallons total (104 diesel } \\
\text { energy equivalent gallons) - 2 LNG } \\
\text { saddle tanks from MVE, Inc. }\end{array}$ \\
\hline $\begin{array}{l}\text { Transmission } \\
\text { Manufacturer/Model }\end{array}$ & Fuller RTL12610B,10 speed & Fuller RT11710B, 10 speed \\
\hline Catalytic Converter Used (Y/N) & No & No \\
\hline $\begin{array}{l}\text { Vehicle Cost in Comparison to } \\
\text { Diesel }\end{array}$ & - & $+\$ 35,000$ \\
\hline
\end{tabular}

\section{Duty Cycle Descriptions}

The LNG trucks at Raley's are used for routes in the Sacramento area and nearby suburbs. This has been done at the request of the Sacramento Metropolitan Air Quality Management District to maximize the emissions benefits within the Sacramento Air Basin. The diesel control trucks used in this study traveled on similar terrain, but went farther (outside of the Sacramento area) to make deliveries and pick ups. This decision to operate the LNG trucks only in the Sacramento area has caused the LNG trucks to have significantly lower mileage per calendar month than the diesel control trucks. This difference in mileage for the two fleets is addressed in more detail later.

Each LNG truck at Raley's is commonly used up to six days a week and two shifts per day. The trucks depart the Distribution Center loaded and return nearly empty (returning with empty pallets and spoiled/damaged goods) unless the truck stopped to backhaul goods to the Distribution Center. The trucks are used most heavily during the week or two before holidays such as Christmas, Thanksgiving, or Memorial Day.

Raley's data have been analyzed on a per-trip and per-day basis (shown in Tables 2 and 3 ) as well as overall vehicle usage (mileage) per month (shown in Table 4 and Figure 7). Table 2 shows the trip information before the clean point in the data (before January 1998) and Table 3 shows the results after the clean point in the data (after January 1, 1998). Appendix $C$ shows a more detailed per-trip and perday summary for each vehicle on a monthly basis. The per-trip and per-day data does not indicate reliability. The data shown in the tables are for days that the trucks have been used and does not include any calculation or indication of downtime. A trip is defined as the route for each truck leaving the Distribution Center, making deliveries (or picking up), and returning to the Distribution Center. The 
backhauls are calculated as the total number of backhaul operations divided by the number of trips taken by the fleet. A backhaul calculation of 0.2 indicates that 2 out of 10 trips has a backhaul within the trip.

Table 2. Truck Usage Per Trip and Per Day

Before Clean Point (May 97 - December 97)

\begin{tabular}{|c|c|c|c|c|c|c|c|c|}
\hline \multirow{2}{*}{ Vehicle } & \multicolumn{4}{|c|}{ Average Per Trip } & \multicolumn{4}{c|}{ Average Per Day } \\
\cline { 2 - 9 } & Miles & Hours & Stores & Back Haul & Miles & Hours & Stores & Back Haul \\
\hline 1501 & 50.5 & 3.6 & 2.1 & 0.1 & 146.4 & 10.4 & 6.0 & 0.2 \\
\hline 1502 & 59.2 & 3.9 & 2.3 & 0.2 & 171.5 & 11.3 & 6.5 & 0.5 \\
\hline 1503 & 67.2 & 4.2 & 2.4 & 0.1 & 189.9 & 11.9 & 6.7 & 0.3 \\
\hline 1504 & 61.9 & 3.9 & 2.1 & 0.1 & 189.6 & 12.0 & 6.6 & 0.3 \\
\hline 1505 & 47.9 & 3.7 & 2.3 & 0.1 & 136.2 & 10.7 & 6.5 & 0.4 \\
\hline 1506 & 59.8 & 4.0 & 2.4 & 0.2 & 178.1 & 11.9 & 7.0 & 0.7 \\
\hline 1507 & 102.6 & 5.2 & 2.8 & 0.3 & 242.7 & 12.4 & 6.6 & 0.6 \\
\hline 1508 & 68.6 & 4.1 & 2.1 & 0.1 & 210.9 & 12.6 & 6.6 & 0.4 \\
\hline LNG & $\mathbf{6 3 . 8}$ & 4.1 & 2.3 & $\mathbf{0 . 2}$ & 183.5 & 11.7 & $\mathbf{6 . 6}$ & $\mathbf{0 . 4}$ \\
\hline 1586 & 188.5 & 8.0 & 3.5 & 1.0 & 233.5 & 9.9 & 4.3 & 1.2 \\
\hline 1592 & 226.1 & 9.9 & 4.3 & 1.6 & 232.9 & 10.2 & 4.4 & 1.6 \\
\hline 1593 & 212.6 & 9.0 & 3.9 & 1.3 & 250.3 & 10.6 & 4.6 & 1.5 \\
\hline Diesel & 208.2 & 8.9 & 3.9 & $\mathbf{1 . 3}$ & 238.8 & 10.2 & 4.4 & 1.5 \\
\hline
\end{tabular}

Table 3. Truck Usage Per Trip and Per Day

After Clean Point (January 98 - December 98)

\begin{tabular}{|c|c|c|c|c|c|c|c|c|}
\hline \multirow{2}{*}{ Vehicle } & \multicolumn{4}{|c|}{ Average Per Trip } & \multicolumn{4}{c|}{ Average Per Day } \\
\cline { 2 - 9 } & Miles & Hours & Stores & Back Haul & Miles & Hours & Stores & Back Haul \\
\hline 1501 & 57.2 & 3.9 & 2.3 & 0.1 & 157.5 & 10.6 & 6.3 & 0.2 \\
\hline 1502 & 67.9 & 4.1 & 2.3 & 0.1 & 185.3 & 11.2 & 6.3 & 0.3 \\
\hline 1503 & 71.3 & 4.3 & 2.3 & 0.1 & 210.2 & 12.6 & 6.9 & 0.3 \\
\hline 1504 & 77.1 & 4.6 & 2.5 & 0.2 & 233.4 & 13.8 & 7.6 & 0.5 \\
\hline 1505 & 76.3 & 4.5 & 2.4 & 0.1 & 192.3 & 11.2 & 5.9 & 0.3 \\
\hline 1506 & 62.0 & 4.1 & 2.4 & 0.1 & 177.4 & 11.8 & 6.8 & 0.3 \\
\hline 1507 & 70.4 & 4.3 & 2.4 & 0.1 & 202.3 & 12.3 & 6.8 & 0.3 \\
\hline 1508 & 78.7 & 4.4 & 2.2 & 0.1 & 198.5 & 11.0 & 5.6 & 0.3 \\
\hline LNG & 69.9 & 4.3 & 2.4 & $\mathbf{0 . 1}$ & 194.8 & 11.9 & 6.6 & $\mathbf{0 . 3}$ \\
\hline 1586 & 220.2 & 8.2 & 3.1 & 0.3 & 287.9 & 10.7 & 4.1 & 0.4 \\
\hline 1592 & 185.4 & 8.5 & 3.7 & 1.2 & 215.4 & 9.9 & 4.3 & 1.4 \\
\hline 1593 & 230.1 & 9.2 & 3.9 & 0.8 & 265.5 & 10.6 & 4.4 & 0.9 \\
\hline Diesel & $\mathbf{2 1 1 . 8}$ & $\mathbf{8 . 6}$ & $\mathbf{3 . 5}$ & $\mathbf{0 . 8}$ & $\mathbf{2 5 5 . 8}$ & $\mathbf{1 0 . 4}$ & $\mathbf{4 . 3}$ & $\mathbf{0 . 9}$ \\
\hline
\end{tabular}


Table 4. Average Monthly Mileage

\begin{tabular}{|c|c|c|c|c|c|c|}
\hline \multirow{2}{*}{ Vehicle } & \multicolumn{3}{|c|}{ Before Clean Point (May 97-Dec 97) } & \multicolumn{2}{c|}{ After Clean Point (Jan 98-Dec 98) } \\
\cline { 2 - 7 } & Mileage & Months & Monthly Avg & Mileage & Months & Monthly Avg \\
\hline 1501 & 31,948 & 8 & 3,994 & 46,050 & 12 & 3,838 \\
\hline 1502 & 30,922 & 8 & 3,865 & 55,833 & 12 & 4,653 \\
\hline 1503 & 36,054 & 8 & 4,507 & 50,842 & 12 & 4,237 \\
\hline 1504 & 40,716 & 8 & 5,090 & 67,580 & 12 & 5,632 \\
\hline 1505 & 24,408 & 8 & 3,051 & 52,334 & 12 & 4,361 \\
\hline 1506 & 34,848 & 8 & 4,356 & 52,460 & 12 & 4,372 \\
\hline 1507 & 37,905 & 8 & 4,738 & 55,855 & 12 & 4,655 \\
\hline 1508 & 40,954 & 8 & 5,119 & 50,014 & 12 & 4,168 \\
\hline LNG & $\mathbf{2 7 7 , 7 5 5}$ & $\mathbf{6 4}$ & $\mathbf{4 , 3 4 0}$ & $\mathbf{4 3 0 , 9 6 8}$ & 96 & 4,489 \\
\hline 1586 & 37,115 & 6 & 6,186 & 83,488 & 12 & 6,957 \\
\hline 1592 & 35,420 & 6 & 5,903 & 64,889 & 12 & 5,407 \\
\hline 1593 & 36,424 & 6 & 6,071 & 74,186 & 12 & 6,182 \\
\hline Diesel & 108,959 & $\mathbf{1 8}$ & $\mathbf{6 , 0 5 3}$ & $\mathbf{2 2 2 , 5 6 3}$ & $\mathbf{3 6}$ & $\mathbf{6 , 1 8 2}$ \\
\hline
\end{tabular}

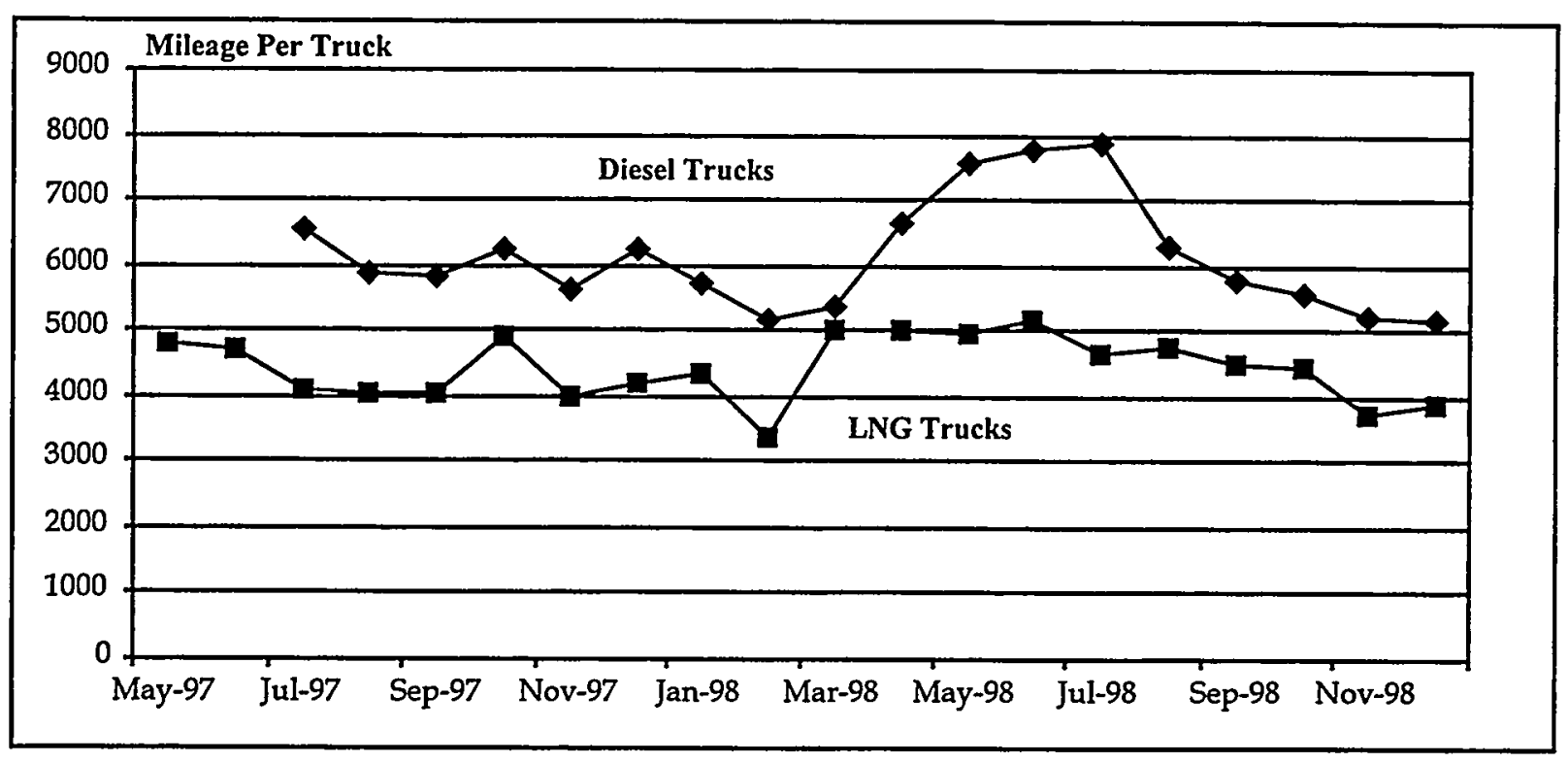

Figure 7. Average Monthly Mileage Per Truck

The statistics in Table 3 show that each LNG truck is used on three separate trips in a day. The LNG trucks operate nearly 12 hours per day, deliveries are made to (or from) 6 to 7 stores, mileage per day averages 195 miles per truck, and a backhaul is made on every third truck on any given day of operation. The diesel trucks since the clean point were used mostly on one route in a given day with another very short route, in other words, there is little difference between the per-trip mileage and the per-day mileage. 
The diesel trucks averaged 10 hours, 4 to 5 stores, and one backhaul daily per truck. The average mileage per day for the diesel trucks was 256 miles per truck.

For comparing before and after the clean point in the data, the LNG trucks have increased in mileage per day by 6 percent and the hours of operation have increased slightly. The diesel control trucks have had a 7 percent increase in mileage per day, the hours of operation have increased slightly, but the backhauls have decreased by one-third. Both the LNG and diesel trucks have increased usage, most likely from the increase in number of stores in the Raley's operation with the acquisition of Nob Hill in mid-1998.

At the start of operation of the LNG trucks (May-July 1997), a study of average vehicle speed during vehicle operation (excluding time at the loading docks) was completed. Raley's trucks have an onboard computer monitoring system from Cadec. This monitoring system tracks vehicle operation, idle time, and the stores that are serviced by the truck. The average vehicle speeds were $45 \mathrm{mph}$ for the diesel control trucks and $39 \mathrm{mph}$ for the LNG trucks. During November 1998, average speeds were measured again. The diesel control trucks showed an average speed of $40 \mathrm{mph}$, and the LNG trucks had an average speed of $37 \mathrm{mph}$. Both groups of trucks had a slightly lower, but similar average speed in November 1998 as compared to results from 1997 . The average speed of the diesel trucks is slightly higher than the LNG trucks. This difference in speed is most likely a result of LNG trucks servicing more stores per day as indicated in the trip data (6.6 average number of stores per day for each LNG truck as compared to 4.2 stores per day for each diesel truck).

Table 4 shows average monthly mileage for each truck in the study for before and after the clean point in the data. Average mileage for both study fleets has increased slightly ( 2 to 3 percent) since the clean point as compared to before the clean point. The LNG study fleet has consistently been about 27 percent lower for average monthly mileage per truck. Figure 7 shows the average monthly mileage per truck for the LNG and diesel study fleets for the data collection period. This shows that the diesel trucks have consistently averaged higher mileage. Also, between March and August 1998, the LNG trucks have consistently averaged 5,000 miles and the diesel trucks have had the average mileage increase significantly to nearly 8,000 miles per month. This change in the diesel truck usage as well as the slight increase in the LNG truck usage is directly related to the addition of the Nob Hill stores into the operation from the Raley's Distribution Center. The average mileage for the diesel trucks had come back down to about 5,000 miles per truck by the end of the data collection period. This occurred due to more trucks being added to the fleet to better accommodate the Nob Hill stores.

The LNG and diesel trucks saw significantly different usage during the evaluation, based on the trip, average speed data, and monthly mileage. These differences impact fuel economy and wear and tear on the vehicles. The in-use fuel economy results have been compared to the emissions testing fuel economy results to help resolve the differences in duty cycle of the in-use data. The maintenance data analysis has been used to investigate the difference in the mileage base by analyzing cost per mile and cost per truck for the same length of data period. 


\section{Operational Results: \\ Fuel Consumption, Engine Oil Consumption, and Maintenance Costs}

\section{Data Collection Methods}

Data were gathered from Raley's and Ozark Trucking's fuel and maintenance tracking systems on a daily basis and then transferred to Battelle on a monthly basis. Diesel fuel data were collected electronically from the on-site fueling station, and LNG fuel data were collected on $\log$ forms at the on-site fueling station. Diesel and LNG fuel data were transferred to Battelle with the daily dispatching log (truck assignments for each day). Engine oil additions were tracked in the fuel tracking systems. Fuel cost data were provided for diesel fuel electronically with the diesel log forms, and LNG costs were provided in the form of the actual invoices from the LNG fuel provider. Maintenance data were provided by Ozark Trucking on a quarterly basis after each of the study trucks was serviced for scheduled preventive maintenance. Copies of the actual maintenance work order forms and supporting paperwork were sent to Battelle.

Data from Raley's and Ozark Trucking were processed and analyzed soon after receipt at Battelle. Any potentially missing or incorrect data were investigated through the analysis and then in discussions with Raley's and/or Ozark Trucking. On a regular basis during the data collection period, updated analyses were provided to Raley's and Ozark Trucking for review and comment.

Vehicle operational data were collected at each fuel fill, at each change in fuel price, at each addition of engine oil, and at each oil change. The following values were recorded for analysis:

- Fuel fill - amount of fuel, odometer reading, date

- Fuel price change - type of fuel, price, date

- Addition of engine oil - amount of oil, odometer reading, date

- Oil change (usually within scope of preventive maintenance) - amount of oil, odometer reading, date.

Maintenance data were collected at each repair action, such as preventive maintenance, unscheduled maintenance, road calls, and warranty repair actions. The following values were recorded for analysis:

- Date of repair, labor hours, number of days out of service, odometer reading, parts replaced, parts cost, and descriptions of problem reported and actual repair performed.

Maintenance cost data were used (along with fuel and engine oil consumption costs) to estimate operating costs and as a measure of reliability and durability. Technical information on repairs covered by the vehicle warranties was collected primarily for indications of reliability and durability. Warranty repair costs (i.e., those costs reimbursed by the manufacturer), however, were considered to have been included 
in the purchase price of the vehicle and were not included in the cost analysis. Labor costs for warranty repairs were included, if Ozark Trucking's mechanic did the work and those hours were not reimbursed under the warranty agreement.

Safety incidents affecting the vehicles or occurring at Raley's fueling station or in the maintenance facilities were planned to be tracked, including a description of the nature of the incident or accident and the vehicles or facilities involved. Also, records were planned to be made of any changes in procedures or hardware required to help prevent future safety incidents. However, no safety incidents were reported during the data collection period.

\section{Summary of Operational Results}

The following sections summarize the operational results of the evaluation. Detailed data analyses are presented in the following appendices:

- Appendix D - Statistics of all operational costs for the LNG and diesel control trucks

- Appendix E-Fuel and engine oil consumption and fuel economy by truck and month

- Appendix F - LNG and diesel fuel costs

- Appendix G - Monthly maintenance costs by truck.

\section{Fuel Consumption, Fuel Economy, and Cost}

LNG Fuel Measurement and Losses to Atmosphere - Measurement of LNG fuel has been an issue since the beginning of LNG truck operations at Raley's. The temporary fueling station, or QRS, was filled by a partial tanker truckload of LNG. When the tanker truck unloads the LNG, pressure at the station must be reduced so that the fuel will flow from the tanker truck to the LNG station. This depressurization required a significant amount of natural gas to be vented to the atmosphere.

Raley's paid for all of the fuel offloaded from the tanker truck, including the vented fuel from the station. The fuel lost from the refueling station was not measured. This venting did not affect the measurement of LNG consumed by Raley's trucks participating in the evaluation. However, the cost that Raley's paid for the LNG fuel in reality was slightly higher than the fuel cost as documented, because the natural gas lost to atmosphere was not available for use by the fleet. This loss from the QRS was probably on the order of $\$ 0.01$ per gallon of LNG.

LNG fuel was also lost to atmosphere during truck refueling. To speed up the fueling process of the LNG-powered trucks, the vent on each truck's fuel tank was held open during the fueling process (vent filling). The vent released natural gas from the fuel tank to the atmosphere. This lost vent gas reduced the fuel economy of the truck slightly. More accurate fuel economy results based solely on the LNG engine fuel consumption were not determined in this evaluation. The need for vent filling was removed 
by the use of the permanent LNG fueling station. The amount of the LNG fuel vented to atmosphere during fueling was probably less than one percent of the fuel loaded on-board the truck.

Near the end of the data collection, the permanent LNG station was completed and started operation. The permanent LNG fueling station controlled most of the vent losses during station resupply and truck refueling. The new station was able to keep the LNG fuel colder, and the station operated at lower pressure than the QRS, which reduced venting from the fuel station. The new station was designed to pressure fill the LNG trucks. The pressure to fill the trucks was produced using warmed natural gas from a vaporizer added to a smaller tank with LNG at the station. The pressurized LNG in the smaller tank is then used to fill the LNG trucks at a higher pressure, and therefore faster, than the temporary station without the need to vent fill the trucks. An LNG truck being fueled is shown in Figure 8.

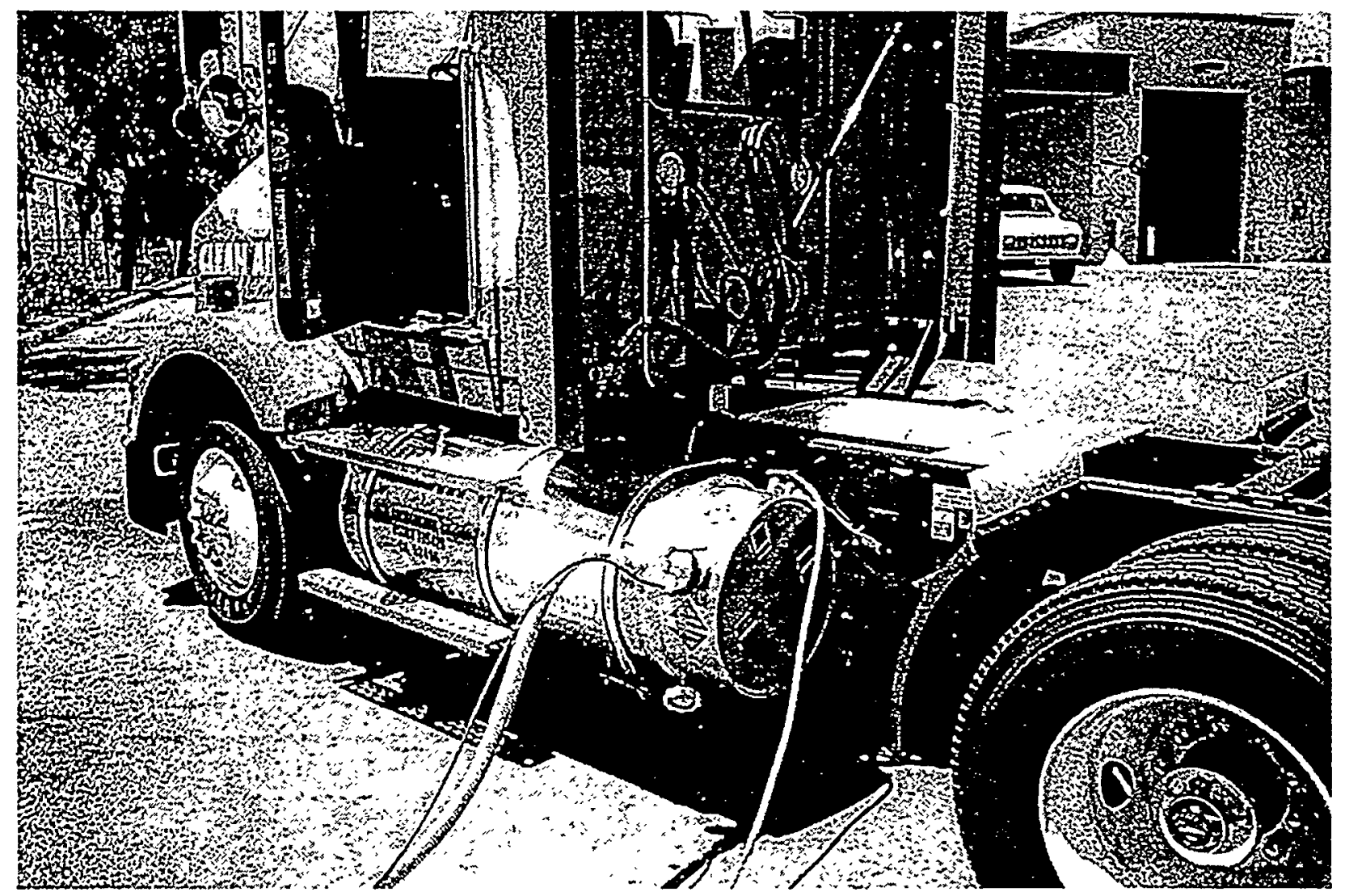

Figure 8. LNG Truck at Raley's Being Fueled

Fuel Consumption and Fuel Economy - Raley's LNG trucks (8 LNG tractors and 2 yard tractors) used 500 to 700 gallons of LNG per work day ( 6 days a week), and each LNG truck used 77 gallons of LNG on average per day of operation. On average, the 3 diesel control trucks used 36 gallons of diesel per day of operation. 
Tables 5 and 6 show fuel consumption and economy for both fleets before the data clean point (April 97 December 97) and after the data clean point (January 98 - December 98). Diesel energy equivalent gallons are calculated based on a standard LNG gallon and divided by 1.67 (the conversion factor for pure methane). The fuel supplier, Jack B. Kelley, for Raley's confirmed that the LNG used during the evaluation was essentially pure methane.

Table 5. Fuel Consumption and Economy

Before Clean Point (April 97 - December 97)

\begin{tabular}{|c|c|c|c|c|c|}
\hline Vehicle & Mileage & LNG Gal. & $\begin{array}{c}\text { Miles/LNG } \\
\text { Gal. }\end{array}$ & $\begin{array}{c}\text { Diesel Energy } \\
\text { Equivalent Gal.* }\end{array}$ & MPEG** $^{*}$ \\
\hline 1501 & 33,241 & 12,401 & 2.68 & 7,426 & 4.48 \\
\hline 1502 & 31,888 & 12,516 & 2.55 & 7,494 & 4.25 \\
\hline 1503 & 35,917 & 13,962 & 2.57 & 8,361 & 4.30 \\
\hline 1504 & 40,085 & 14,437 & 2.78 & 8,645 & 4.64 \\
\hline 1505 & 25,197 & 10,161 & 2.48 & 6,085 & 4.14 \\
\hline 1506 & 34,342 & 13,271 & 2.59 & 7,946 & 4.32 \\
\hline 1507 & 37,150 & 13,814 & 2.69 & 8,272 & 4.49 \\
\hline 1508 & 40,717 & 15,117 & 2.69 & 9,052 & 4.50 \\
\hline LNG & $\mathbf{2 7 8 , 5 3 7}$ & 105,679 & 2.64 & $\mathbf{6 3 , 2 8 1}$ & $\mathbf{4 . 4 0}$ \\
\hline 1586 & 39,463 & - & - & 5,580 & 7.07 \\
\hline 1592 & 38,756 & - & - & 5,419 & 7.15 \\
\hline 1593 & 38,317 & - & - & 5,601 & 6.84 \\
\hline Diesel & $\mathbf{1 1 6 , 5 3 6}$ & - & - & $\mathbf{1 6 , 6 0 0}$ & $\mathbf{7 . 0 2}$ \\
\hline
\end{tabular}

Note: the mileage and LNG gallons columns show the amount used in the calculations, not the total amount used in-service.

* Diesel energy equivalent gallons are calculated by LNG Gal / 1.67

** MPEG - miles per equivalent gallon

Table 6. Fuel Consumption and Economy

After Clean Point (January 98 - December 98)

\begin{tabular}{|c|c|c|c|c|c|}
\hline Vehicle & Mileage & LNG Gal. & $\begin{array}{c}\text { Miles/LNG } \\
\text { Gal. }\end{array}$ & $\begin{array}{c}\text { Diesel Energy } \\
\text { Equivalent Gal.* }\end{array}$ & MPEG** $^{*}$ \\
\hline 1501 & 43,616 & 16,695 & 2.61 & 9,997 & 4.36 \\
\hline 1502 & 51,552 & 19,283 & 2.67 & 11,547 & 4.46 \\
\hline 1503 & 47,359 & 18,812 & 2.52 & 11,265 & 4.20 \\
\hline 1504 & 60,416 & 23,009 & 2.63 & 13,778 & 4.39 \\
\hline 1505 & 48,066 & 18,907 & 2.54 & 11,322 & 4.25 \\
\hline 1506 & 48,446 & 19,152 & 2.53 & 11,468 & 4.22 \\
\hline 1507 & 51,982 & 20,284 & 2.56 & 12,146 & 4.28 \\
\hline 1508 & 44,680 & 16,944 & 2.64 & 10,146 & 4.40 \\
\hline LNG & 396,118 & 153,084 & 2.59 & 91,667 & 4.32 \\
\hline 1586 & 75,173 & - & - & 10,703 & 7.02 \\
\hline 1592 & 58,554 & - & - & 8,384 & 6.98 \\
\hline 1593 & 67,234 & - & - & 9,544 & 7.04 \\
\hline Diesel & 200,961 & - & - & 28,630 & 7.02 \\
\hline
\end{tabular}

Note: the mileage and LNG gallons columns show the amount used in the calculations, not the total amount used in-service.

* Diesel energy equivalent gallons are calculated by LNG Gal / 1.67

** MPEG - miles per equivalent galion 
The fuel consumption was higher for the LNG trucks than for the diesel trucks. Before the clean point, the average energy equivalent fuel economy was 37 percent lower for the LNG trucks than for the diesel trucks. After the clean point, the difference in the fuel economies changed slightly to 38 percent lower.

The energy equivalent fuel economy of the LNG trucks was not expected to be 37 to 38 percent lower than the diesel trucks. In other heavy-duty applications of the L10 natural gas engine (such as transit buses), the energy equivalent fuel economy difference was 20 to 25 percent lower compared to similar diesel engines. Fuel economy measurements made as part of the emissions testing on a dynamometer (described in detail elsewhere) confirm the difference in fuel economy seen in field operations. Emissions testing was performed on the Raley's study vehicles twice during the data collection period. The two-part emissions test showed the LNG trucks averaging about 33 percent lower energy equivalent fuel economy than the diesel trucks ( 30 percent lower than diesel in the first test, and 35 percent lower in the second test).

Energy equivalent fuel economy for the LNG trucks, as compared with the diesel trucks, was better in the emissions tests than in the field. Several factors may explain this difference. In general, since the emissions testing is a controlled test with a specific duty cycle, this will be somewhat to significantly different in comparison to the in-use duty cycle of the trucks both in acceleration profiles and average speed. For the in-use fuel economy, the measurement of LNG in the fuel tanks and losses from vent filling as discussed earlier in this section, may also have affected the fuel consumption results.

Overall, the fuel economies from the emissions testing and in-use data in regards to difference between the LNG and diesel control trucks are similar.

Fuel Costs - With taxes, the average LNG cost was much higher than the average diesel cost. Raley's LNG was delivered to Sacramento by Jack B. Kelley from supplies in Wyoming and Arizona. Transportation costs (based on distance to be trucked) for LNG (or any commodity being trucked) are a significant portion of the overall cost. Also, during the early part of the evaluation, Raley's purchased LNG in quantities less than a full truckload, because of the limited capacity of the QRS (5,000 gallons). Transportation costs for a partial truckload of LNG were the same as those for a full truckload $(10,000$ gallons), so the cost per gallon of LNG delivered to the QRS was much higher than the cost per gallon using the permanent fueling station. The permanent LNG station (13,000-gallon total capacity) could receive a full truckload of LNG. For fuel delivery to the QRS (partial truckload), the LNG cost was $\$ 0.575$ per gallon plus tax by contract after April 1998; with the permanent station, the cost per gallon was $\$ 0.475$ plus tax. The LNG fuel cost could be reduced even further if fuel were available from a source closer to Raley's because the transportation costs would be reduced significantly.

During the data collection period, diesel costs started high, as high as $\$ 1.29$ per gallon including tax, and dropped to very low levels, as low as $\$ 0.94$ per gallon including tax. Table 7 shows monthly average fuel costs paid for LNG (with and without tax) and diesel (with tax). Federal tax rates for LNG changed during the data collection period. As shown in Table 7, the LNG diesel equivalent cost per gallon with 
tax included the Federal tax for LNG ( $\$ 0.183$ per LNG gallon) in effect through the end of 9/97 and then the new Federal tax for LNG ( $\$ 0.119$ per LNG gallon) beginning in 10/97. Although the diesel costs have been low during most of the data collection period, as of March 31, 1999, the diesel price at Raley's was $\$ 1.38$ per gallon with taxes.

Table 7. Monthly Average Fuel Costs

\begin{tabular}{|c|c|c|c|}
\hline Month & $\begin{array}{c}\text { Avg. LNG Cost } \\
\text { per Gal. (\$) }\end{array}$ & $\begin{array}{c}\text { Avg. LNG Diesel } \\
\text { Equivalent Cost per } \\
\text { Gal. With Tax (\$) }\end{array}$ & $\begin{array}{c}\text { Avg. Diesel Cost per } \\
\text { Gal. With Tax (\$) }\end{array}$ \\
\hline $4 / 97$ & 0.583 & 1.38 & \\
\hline $5 / 97$ & 0.550 & 1.32 & \\
\hline $6 / 97$ & 0.558 & 1.34 & 1.05 \\
\hline $7 / 97$ & 0.550 & 1.32 & 1.20 \\
\hline $8 / 97$ & 0.550 & 1.32 & 1.19 \\
\hline $9 / 97$ & 0.550 & 1.32 & 1.18 \\
\hline $10 / 97$ & 0.550 & 1.22 & 1.23 \\
\hline $11 / 97$ & 0.550 & 1.22 & 1.11 \\
\hline $12 / 97$ & 0.550 & 1.22 & 1.09 \\
\hline $1 / 98$ & 0.550 & 1.22 & 1.03 \\
\hline $2 / 98$ & 0.550 & 1.22 & 0.99 \\
\hline $3 / 98$ & 0.550 & 1.22 & 1.06 \\
\hline $4 / 98$ & 0.562 & 1.24 & 1.03 \\
\hline $5 / 98$ & 0.575 & 1.26 & 0.99 \\
\hline $6 / 98$ & 0.575 & 1.26 & 0.98 \\
\hline $7 / 98$ & 0.595 & 1.29 & 0.99 \\
\hline $8 / 98$ & 0.575 & 1.26 & 1.02 \\
\hline $9 / 98$ & 0.575 & 1.26 & 1.00 \\
\hline $10 / 98$ & 0.575 & 1.26 & 0.99 \\
\hline $11 / 98$ & 0.575 & 1.26 & 0.89 \\
\hline $12 / 98$ & 0.534 & 1.19 & 1.17 \\
\hline $\begin{array}{c}\text { Average - Before } \\
\text { Clean Point }\end{array}$ & 0.554 & 1.29 & 1.01 \\
\hline $\begin{array}{c}\text { Average - After } \\
\text { Clean Point }\end{array}$ & 0.566 & 1.24 & \\
\hline $\begin{array}{c}\text { Cost of LNG at } \\
\text { Lower Rate for } \\
\text { New Station }\end{array}$ & 0.475 & 1.09 & \\
\hline
\end{tabular}

Note: Average LNG cost per gallon reflects any charges from the trucking company for driver or special unloading.

Taxes prior to $10 / 1 / 97$ were $\$ 0.183$ (federal) $+\$ 0.06(\mathrm{CA})=\$ 0.243$ per LNG gal.

Taxes from $10 / 1 / 97$ on were $\$ 0.119$ (federal) $+\$ 0.06(C A)=\$ 0.179$ per LNG gal.

LNG gallons are converted to diesel energy equivalent gallons by multiplying by 1.67 .

Fuel usage cost per mile is derived from the volume of fuel used, the cost of that fuel, and the number of miles that the trucks have traveled using the fuel. In other words, fuel usage cost is a function of the cost of the fuel and the vehicle's fuel economy. As Table 8 shows, the fuel usage cost per mile was much higher for the LNG trucks. As discussed earlier in this section, several factors may have contributed to . 
this higher fuel usage cost for the LNG trucks. First, the fuel economy was 38 percent lower for the LNG fleet on an energy equivalent basis. Second, the fuel cost per gallon on an energy equivalent basis was 10 percent higher for LNG before the clean point ( $\$ 1.29$ per energy equivalent gallon for LNG and $\$ 1.17$ per gallon for diesel) and 23 percent higher after the clean point ( $\$ 1.24$ per energy equivalent gallon for LNG and $\$ 1.01$ per gallon for diesel). Diesel fuel cost was very low in the last 12 months of the data collection period.

\section{Table 8. Fuel Usage Cost Analysis}

\begin{tabular}{|l|c|c|r|r|r|}
\hline \multicolumn{1}{|c|}{ Data Period } & Fleet & Mileage & $\begin{array}{c}\text { Diesel } \\
\text { (Equiv.) Used }\end{array}$ & $\begin{array}{c}\text { Diesel (Equiv.) } \\
\text { Cost/Gal. (\$) }\end{array}$ & $\begin{array}{c}\text { Fuel Usage Cost } \\
\text { per Mile (\$) }\end{array}$ \\
\hline Before Clean Point & LNG & 278,536 & 63,281 & 1.29 & 0.293 \\
(4/97-12/97) & Diesel & 116,536 & 16,599 & 1.17 & 0.167 \\
\hline After Clean Point & LNG & 396,118 & 91,667 & 1.24 & 0.287 \\
(1/98-12/98) & Diesel & 200,961 & 28,630 & 1.01 & 0.144 \\
\hline After Clean Point & LNG & 396,118 & 91,667 & 1.09 & 0.252 \\
Using Current Prices & Diesel & 200,961 & 28,630 & $1.38^{*}$ & 0.197 \\
\hline After Clean Point & LNG & 396,118 & 91,667 & $0.88^{* *}$ & 0.204 \\
Using Potential Prices & Diesel & 200,961 & 28,630 & 1.50 & 0.214 \\
\hline
\end{tabular}

** Local LNG price has been suggested as low as $\$ 0.35$ per LNG gallon

Note: Mileage shown is not all mileage during the data periods, but the portion used for this analysis.

Equation for Fuel Usage Cost per Miles is Diesel (Equiv.) Used * Diesel (Equiv.) Cost/Gal. / Mileage

For data before the clean point, the fuel usage cost per mile was 75 percent higher for LNG. After the clean point, the fuel usage cost per mile was 99 percent higher for LNG. Fuel usage costs for the LNG trucks were much higher after the clean point because of the low diesel fuel cost, which was temporary. Using 3/31/99 prices of fuel and the fuel economy data for after the clean point ( $\$ 1.38$ per diesel gallon and $\$ 1.09$ per diesel equivalent gallon for LNG), the fuel usage costs were $\$ 0.252$ per mile for the LNG trucks and $\$ 0.197$ per mile for the diesel trucks (28 percent higher for LNG).

Another factor affecting fuel cost is local fuel availability. Using small-scale liquefaction for the LNG, prices have been discussed to be as low as $\$ 0.35$ per LNG gallon delivered to Raley's. Also, diesel fuel prices in California have historically been volatile and may go as high as $\$ 1.50$ per gallon in the near future. Using these two potential fuel prices, the fuel usage cost per mile would be 5 percent lower for the LNG trucks.

\section{Engine Oil Consumption and Cost}

Engine oil consumption is the amount of engine oil consumed by the engine, measured by recording the volume of engine oil added between oil changes. For most engines, a certain level of engine oil consumption is expected. Greater than expected engine oil consumption is a precursor to engine 
problems. Engine oil consumption for the LNG trucks at Raley's was recorded; however, engine oil consumption for the diesel trucks was not tracked on an individual truck basis. As indicated in Appendix $E$, the LNG trucks consumed on average 1.14 quarts of engine oil consumed per 1,000 miles before the clean point and 1.02 quarts of engine oil per 1,000 miles after the clean point. Based on discussions with Cummins and Raley's/Ozark Trucking concerning past experience with other heavy-duty engines, engine oil consumption around 1 quart per 1,000 miles is as good as or better than can be expected.

Oil for the LNG engines costs more than twice as much as oil for the diesel engines: $\$ 1.67$ per quart for the LNG engines and $\$ 0.81$ per quart for the diesel engines. The higher cost for the LNG engine oil is due to the low demand required by a small population of heavy-duty natural gas engines and the special (low ash) oil specification. Operating cost comparisons could not be made between LNG and diesel oil usage. Engine oil consumption costs tend to be very small compared to the fuel and maintenance costs; however, the extra cost of the special engine oil is a factor to be considered in the operation of heavy-duty alternative fuel trucks.

\section{Maintenance, Maintenance Costs, and Warranty}

Maintenance and warranty data were collected from Ozark Trucking quarterly for this study, through Ozark Trucking's normal preventive maintenance action (PMA) cycle. The PMAs are numbered sequentially in this analysis starting with the first PMA (truck would be three months old) for each vehicle. All unscheduled maintenance actions were tracked on forms for each vehicle for parts used and labor hours spent on each vehicle. That information was then combined with the PMA forms.

After the vehicles were through a PMA cycle, Ozark Trucking sent Battelle copies of all paperwork (including invoices from Cummins on warranty claims) for each of the study vehicles since the last PMA. Maintenance data analysis included the following:

- General maintenance costs by vehicle and total

- Maintenance costs broken down by vehicle system

- Driver complaints

- Warranty data broken down by month and by issue.

Maintenance Cost Data Summary - Total maintenance costs by truck are shown in Table 9. Because the diesel trucks were 1 year older than the LNG trucks at the time of the evaluation, maintenance data on the diesel trucks were collected back to the beginning of operation (when the trucks were new). Data back to the start of operation for the LNG trucks also were collected. In order to measure differences in maintenance actions and costs between the diesel and LNG fleets, a similar portion of the vehicle lives was chosen for the maintenance analyses.

The PMA milestones have not occurred in the same calendar time frame for the two study fleets (diesel late 95 through mid-97; LNG - Spring 97 through the end of 98). The labor hour cost for maintenance . 
has been held fixed at $\$ 50$ per hour, and the parts costs have been set using a listing of the costs as of $3 / 31 / 99$, so the maintenance cost analysis was performed using constant dollars. PMA 1 has been removed from the data analysis because the first PMA information includes vehicle preparation for operation (painting, putting vehicle numbers on, and putting chrome parts on) and other maintenance actions such as operation start up problems that are not consistent between vehicles.

Table 9 presents data for PMA 2 through 3 and for PMA 4 through 7. PMA 2 through 3 represents approximately 6 months of operation, and PMA 4 through 7 represents approximately 12 months of operation. Maintenance data were also collected between the PMA milestones, including all unscheduled maintenance actions. Warranty actions not paid for by the trucking company were not included in the cost analysis, but are addressed separately later in this section.

Table 9. Maintenance Costs

\begin{tabular}{|c|c|c|c|c|c|c|c|c|}
\hline \multirow[b]{2}{*}{ Vehicle } & \multicolumn{4}{|c|}{ PMA 2 Through 3} & \multicolumn{4}{|c|}{ PMA 4 Through 7} \\
\hline & Mileage & Parts (\$) & $\begin{array}{l}\text { Labor } \\
\text { Hrs. }\end{array}$ & $\begin{array}{l}\text { Cost (\$) } \\
\text { per Mile }\end{array}$ & Mileage & Parts (\$) & $\begin{array}{l}\text { Labor } \\
\text { Hrs. }\end{array}$ & $\begin{array}{l}\text { Cost (\$) } \\
\text { per Mile }\end{array}$ \\
\hline 1501 & 24,941 & 415.72 & 26.1 & 0.069 & 46,032 & $3,100.71$ & 37.8 & 0.108 \\
\hline 1502 & 20,568 & 382.25 & 24.8 & 0.079 & 56,275 & $2,275.24$ & 34.5 & 0.071 \\
\hline 1503 & 26,920 & 759.49 & 30.8 & 0.085 & 53,665 & $3,678.21$ & 48.0 & 0.113 \\
\hline 1504 & 27,417 & $1,499.87$ & 18.9 & 0.089 & 65,853 & $2,718.03$ & 40.7 & 0.072 \\
\hline 1505 & 18,394 & $1,276.18$ & 21.8 & 0.129 & 52,663 & $3,361.31$ & 39.6 & 0.101 \\
\hline 1506 & 23,715 & 571.26 & 18.3 & 0.063 & 54,206 & $4,404.40$ & 40.1 & 0.118 \\
\hline 1507 & 25,991 & 301.61 & 24.1 & 0.058 & 58,519 & $3,242.06$ & 47.2 & 0.096 \\
\hline 1508 & 30,690 & 569.48 & 18.4 & 0.049 & 50,978 & $2,601.40$ & 43.8 & 0.094 \\
\hline$\overline{\text { LNG }}$ & 198,636 & $5,775.86$ & 183.2 & 0.075 & 438,191 & $25,381.36$ & 331.7 & 0.096 \\
\hline $\begin{array}{l}\text { Avg. per } \\
\text { Truck }\end{array}$ & 24,830 & 721.98 & 22.9 & - & 54,774 & $3,172.67$ & 41.5 & - \\
\hline 1586 & 38,901 & 284.49 & 18.0 & 0.030 & 72,980 & $1,487.00$ & 32.1 & 0.042 \\
\hline 1592 & 32,167 & 455.37 & 18.9 & 0.044 & 67,552 & 928.11 & 32.3 & 0.038 \\
\hline 1593 & 39,133 & 243.63 & 18.6 & 0.030 & 82,312 & $3,048.96$ & 39.1 & 0.061 \\
\hline Diesel & 110,201 & 983.49 & 55.5 & 0.034 & 222,844 & $5,464.09$ & 103.5 & 0.048 \\
\hline $\begin{array}{c}\text { Avg. per } \\
\text { Truck }\end{array}$ & 36,734 & 327.83 & 18.5 & - & 74,281 & $1,821.36$ & 34.5 & - \\
\hline $\begin{array}{c}\text { Percent } \\
\text { Difference }\end{array}$ & -32 & +120 & +24 & - & -26 & +74 & +20 & - \\
\hline
\end{tabular}

Note: LNG and diesel data are for the same life of the vehicles - preventive maintenance actions (PMA) 2 through 3 and PMA 4 through 7. These periods are 6-months and 12-months long respectively and represent the second through seventh time the vehicles were brought in for PMA service. This also includes all unscheduled maintenance during each PMA period except for warranty actions.

Labor rate used for calculations was $\$ 50$ per hour. Cost per mile calculation is (Part Cost + (Labor Hrs * 50)) / Mileage. Percent difference is calculated by (LNG number/Diesel number - 1 ) * 100

The major indicator for this maintenance data analysis is cost. Higher cost for a given vehicle system in one fleet is an indicator that potentially there is a reliability and/or maintainability issue. The significant difference in usage between diesel and LNG vehicles in the field affects maintenance cost data and . 
understanding reliability of the LNG trucks because the costs per mile are biased higher. The LNG and diesel trucks operated on Raley's routes and met Raley's trucking needs. The study trucks have been used on similar terrain; however, the diesel trucks saw significantly higher mileage because of their longer routes (and range). This analysis has been based on the same time frame in the life of the trucks and on similar calendar duration. Cost per mile is also shown for comparison.

For PMA 2 through 3, the LNG trucks had 32 percent lower mileage per truck for the 6-month data period. For PMA 4 through 7, the mileage difference was smaller but still significant at 26 percent lower per truck for the LNG vehicles. The decrease in the difference in mileage between the diesel and LNG trucks (6 percent) over time shows an increase in Raley's confidence in operating the LNG trucks after working through start-up problems with the trucks and learning the actual range of the trucks.

For PMA 2 through 3, the maintenance costs for parts per truck were much higher for the LNG trucks (120 percent) and the labor hours were higher (24 percent). The higher parts costs for the LNG trucks were expected because of the higher cost for the engine oil, fuel filter, and spark plugs. The higher labor hours per truck were caused by time spent troubleshooting and repairing fuel leaks, running out of fuel, and repairing rough-running engines.

For PMA 4 through 7, the maintenance costs for parts per truck continued to be higher for the LNG trucks (74 percent), but not to the level seen in PMA 2 through 3. This reflects a drop in parts usage and costs. The labor hours were higher for the LNG trucks for PMA 4 through 7 (20 percent). The higher labor and parts cost per truck were caused by the fuel system (leak repair, leak detection system repair, and higher cost of fuel filters), non-lighting electrical (spark plugs and wires), and engine (rough running and oil changes).

Table 10 shows maintenance costs by vehicle system. The vehicle systems shown in Table 10 include the following:

- Engine/fuel-related systems - exhaust, fuel, engine, electrical minus lighting, air intake, and cooling

- PMA inspections

- Cab, body, and accessories systems - cab and sheet metal, accessories such as phones and fifth wheel, and body

- Frame, steering, and suspension systems

- Axle, wheel, and drive shaft systems

- Tires

- Lighting.

\section{Discussion of Maintenance Costs}

The discussion presented here is based on the cost per truck results in the categories shown in Table 10. 
Table 10. Maintenance Costs by Vehicle System

\begin{tabular}{|c|c|c|c|c|}
\hline \multirow{2}{*}{ Maintenance System Costs } & \multicolumn{2}{|c|}{ PMA 2 Through 3 (6 Months) } & \multicolumn{2}{|c|}{ PMA 4 Through 7 (12 Months) } \\
\hline & LNG & Diesel & LNG & Diesel \\
\hline Mileage Base Used & 198,636 & 110,201 & 438,191 & $\overline{222,844}$ \\
\hline \multicolumn{5}{|c|}{ Total Engine/Fuel-Related Systems (VMRS Codes $30,31,32,33,41,42,43,44,45$ ) } \\
\hline Parts Cost (\$) & $3,561.21$ & 456.90 & $13,103.31$ & $1,328.30$ \\
\hline Labor Hours & 65.8 & 9.1 & 67.7 & 3.4 \\
\hline Total Cost (\$) & $6,851.21$ & 911.90 & $16,488.31$ & $1,498.30$ \\
\hline Total Cost (\$) per Truck & 856.40 & 303.97 & $2,061.04$ & 499.43 \\
\hline Total Cost (\$) per Mile & 0.0345 & 0.0083 & 0.0376 & 0.0067 \\
\hline \multicolumn{5}{|c|}{ Exhaust System Repairs (VMRS Code 43) } \\
\hline Parts Cost (\$) & 16.58 & 25.50 & 0.00 & 25.50 \\
\hline Labor Hours & 1.3 & 0.5 & $\overline{0.8}$ & 0.5 \\
\hline Total Cost (\$) & 81.58 & 50.50 & 40.00 & 50.50 \\
\hline Total Cost (\$) per Truck & 10.20 & 16.83 & 5.00 & 16.83 \\
\hline Total Cost (\$) per Mile & 0.0004 & 0.0005 & 0.0001 & 0.0002 \\
\hline \multicolumn{5}{|c|}{ Fuel System Repairs (VMRS Code 44) } \\
\hline Parts Cost (\$) & $1,059.58$ & 39.90 & $1,905.58$ & 446.53 \\
\hline Labor Hours & 31.7 & 0.8 & 43.2 & 1.3 \\
\hline Total Cost (\$) & $2,644.58$ & 79.90 & $4,065.58$ & 511.53 \\
\hline Total Cost (\$) per Truck & 330.57 & 26.63 & 508.20 & 170.51 \\
\hline Total Cost (\$) per Mile & 0.0133 & 0.0007 & 0.0093 & 0.0023 \\
\hline \multicolumn{5}{|c|}{ Engine System Repairs (VMRS Code 45) } \\
\hline Parts Cost (\$) & $1,847.05$ & 391.50 & $3,760.66$ & 802.50 \\
\hline Labor Hours & 24.8 & 7.3 & 8.4 & 0.6 \\
\hline Total Cost (\$) & $3,087.05$ & 756.50 & $4,180.66$ & 832.50 \\
\hline Total Cost (\$) per Truck & 385.88 & 252.17 & 522.58 & 277.50 \\
\hline Total Cost (\$) per Mile & 0.0155 & 0.0069 & 0.0095 & 0.0037 \\
\hline \multicolumn{5}{|c|}{ Electrical System Repairs (VMRS Codes 30-General Electrical, 31-Charging, 32-Cranking, 33-Ignition) } \\
\hline Parts Cost (\$) & 610.19 & 0.00 & $7,381 . \overline{45}$ & 44.50 \\
\hline Labor Hours & 7.2 & 0.5 & 13.7 & 0.5 \\
\hline Total Cost (\$) & 970.19 & 25.00 & $8,066.45$ & 69.50 \\
\hline Total Cost (\$) per Truck & 121.27 & 8.33 & $1,008.31$ & 23.17 \\
\hline Total Cost (\$) per Mile & 0.0049 & 0.0002 & 0.0184 & 0.0003 \\
\hline \multicolumn{5}{|c|}{ Air Intake System Repairs (VMRS Code 41) } \\
\hline Parts Cost (\$) & 0.00 & 0.00 & 0.00 & 0.00 \\
\hline Labor Hours & 0.0 & 0.0 & 0.0 & 0.0 \\
\hline Total Cost (\$) & 0.00 & 0.00 & 0.00 & 0.00 \\
\hline Total Cost (\$) per Truck & 0.00 & 0.00 & 0.00 & 0.00 \\
\hline Total Cost (\$) per Mile & 0.0000 & 0.0000 & 0.0000 & 0.0000 \\
\hline \multicolumn{5}{|c|}{ Cooling System Repairs (VMRS Code 42) } \\
\hline Parts Cost (\$) & 27.81 & 0.00 & 55.62 & 9.27 \\
\hline Labor Hours & 0.8 & 0.0 & 1.6 & 0.5 \\
\hline Total Cost (\$) & 67.81 & 0.00 & 135.62 & 34.27 \\
\hline Total Cost (\$) per Truck & 8.48 & 0.00 & 16.95 & 11.42 \\
\hline Total Cost (\$) per Mile & 0.0003 & 0.0000 & 0.0003 & 0.0002 \\
\hline
\end{tabular}


Table 10. Maintenance Costs by Vehicle System (continued)

\begin{tabular}{|c|c|c|c|c|}
\hline \multirow{2}{*}{ Maintenance System Costs } & \multicolumn{2}{|c|}{ PMA 2 Through 3 (6 Months) } & \multicolumn{2}{|c|}{ PMA 4 Through 7 (12 Months) } \\
\hline & LNG & Diesel & LNG & Diesel \\
\hline Mileage & 198,636 & 110,201 & 438,191 & 222,844 \\
\hline \multicolumn{5}{|c|}{ PMA Inspections - no parts replacements (101) } \\
\hline Parts Cost (\$) & 0.00 & 0.00 & 0.00 & 0.00 \\
\hline Labor Hours & 73.5 & 30.0 & 145.6 & 60.0 \\
\hline Total Cost (\$) & $3,675.00$ & $1,500.00$ & $7,280.00$ & $3,000.00$ \\
\hline Total Cost (\$) per Truck & 459.38 & 500.00 & 910.00 & $1,000.00$ \\
\hline Total Cost (\$) per Mile & 0.0185 & 0.0136 & 0.0166 & 0.0135 \\
\hline \multicolumn{5}{|c|}{$\begin{array}{l}\text { Cab, Body, and Accessories Systems Repairs (VMRS Codes 02-Cab and Sheet Metal, 50-Accessories, } \\
\text { 71-Body) }\end{array}$} \\
\hline Parts Cost (\$) & $1,450 . \overline{79}$ & 209.76 & $2,211.38$ & 694.31 \\
\hline Labor Hours & 24.4 & 7.0 & 51.0 & 18.5 \\
\hline Total Cost (\$) & $2,670.79$ & 559.76 & $4,761.38$ & $1,619.31$ \\
\hline Total Cost (\$) per Truck & 333.85 & 186.59 & 595.17 & 539.77 \\
\hline Total Cost (\$) per Mile & 0.0134 & 0.0051 & 0.0109 & 0.0073 \\
\hline \multicolumn{5}{|c|}{ Frame, Steering, and Suspension System Repairs (VMRS Codes 14-Frame, 15-Steering, 16-Suspension) } \\
\hline Parts Cost (\$) & 80.00 & 30.00 & 317.34 & 105.32 \\
\hline Labor Hours & 0.0 & 1.3 & 3.7 & 2.3 \\
\hline Total Cost (\$) & 80.00 & 95.00 & 502.34 & 220.32 \\
\hline Total Cost (\$) per Truck & 10.00 & 31.67 & 62.79 & $73 . \overline{44}$ \\
\hline Total Cost (\$) per Mile & 0.0004 & 0.0009 & 0.0011 & 0.0010 \\
\hline \multicolumn{5}{|c|}{$\begin{array}{l}\text { Axle, Wheel, and Drive Shaft System Repairs (VMRS Codes 11-Front Axle, 18-Wheel, 22-Rear Axle, } \\
\text { 24-Drive Shaft) }\end{array}$} \\
\hline Parts Cost (\$) & 0.60 & 0.00 & 20.14 & 190.00 \\
\hline Labor Hours & 0.8 & 0.0 & 0.0 & 0.0 \\
\hline Total Cost $(\$)$ & 40.60 & 0.00 & 20.14 & 190.00 \\
\hline Total Cost (\$) per Truck & 5.08 & 0.00 & 2.52 & 63.33 \\
\hline Total Cost (\$) per Mile & 0.0002 & 0.0000 & 0.0000 & 0.0009 \\
\hline \multicolumn{5}{|c|}{ Tire System Repairs (VMRS Code 17) } \\
\hline Parts Cost (\$) & 480.00 & 215.00 & $9,108.50$ & $2,635.00$ \\
\hline Labor Hours & 6.3 & 2.4 & 21.4 & 9.3 \\
\hline Total Cost (\$) & 795.00 & 335.00 & $10,178.50$ & $3,100.00$ \\
\hline Total Cost (\$) per Truck & 99.38 & 111.67 & $1,272.31$ & $1,033.33$ \\
\hline Total Cost (\$) per Mile & 0.0040 & 0.0030 & 0.0232 & 0.0139 \\
\hline \multicolumn{5}{|c|}{ Lighting System Repairs (VMRS Code 34) } \\
\hline Parts Cost (\$) & 133.98 & 44.00 & 356.76 & 146.01 \\
\hline Labor Hours & 8.8 & 4.1 & 21.8 & 5.8 \\
\hline Total Cost (\$) & 573.98 & 249.00 & $1,446.76$ & 436.01 \\
\hline Total Cost (\$) per Truck & 71.75 & 83.00 & 180.85 & 145.34 \\
\hline Total Cost (\$) per Mile & 0.0029 & 0.0023 & 0.0033 & 0.0020 \\
\hline
\end{tabular}

VMRS - vehicle maintenance reporting system codes from American Trucking Associations.

Engine/Fuel-Related Systems - Parts and labor hours per truck were consistently much higher for the LNG trucks. The low number of labor hours for the diesel trucks showed that labor for these systems for the diesel trucks was covered almost exclusively by the PMA inspection hours. For exhaust repairs, the diesel control cost per truck was higher. For the fuel system, the LNG cost per truck was much higher 
than the diesel trucks. These higher costs for the LNG trucks were made up of LNG tank problems and leaks, fuel gauge complaints, fuel leak sensors, throttle pedal issues, fuel filter cost (\$25 for LNG vs. \$7 for diesel), and cases when LNG trucks ran out of fuel, including towing costs. The engine system repairs were nearly double for the LNG trucks. These extra costs were due to the higher cost of the engine oil ( $\$ 1.67$ per quart for LNG vs. $\$ 0.81$ per quart for diesel) for the LNG trucks and the labor for troubleshooting low power and rough running issues for the LNG trucks. The electrical system repair costs were also much higher for the LNG trucks. For PMA 4 through 7, the higher costs were based on 15 sets of six spark plugs (all for PMA) being replaced for the LNG trucks at a cost of $\$ 164$ per set of spark plugs. There also were four sets of spark plug wires (6 per set) replaced as part of PMAs for $\$ 709$ per set. There were no costs for air intake repairs for either study fleet. Cooling system repair costs were low and about the same for both fleets.

PMA Inspections - This category consists of labor for PMA inspections of the vehicles. On a per-truck -basis, the costs were essentially the same for the PMA 2 through 3 and PMA 4 through 7 data periods. This indicates that neither set of trucks was getting more or less PMA inspection time.

Cab, Body, and Accessories Systems - The LNG and diesel trucks had about the same cost per truck.

Frame, Steering, and Suspension Systems - The diesel trucks were higher in per-truck costs. Each fleet had one leveling valve replaced and a few labor hours.

Axle, Wheel, and Driveshaft Systems - The diesel trucks also had higher per-truck costs for these repairs, because two of the diesel trucks had front end alignments done at $\$ 95$ each.

Tires - Costs for both fleets were high, but close to the same on a per-truck basis. The LNG trucks had a slightly higher cost per truck for tires, possibly due to more non-highway driving based on the higher number of stores per day.

Lighting System - Costs were approximately the same for both fleets on a per-truck basis.

\section{Driver Comments on Vehicles}

As part of the data collection at Raley's, driver complaints were collected for the LNG and diesel trucks. Raley's policy is to collect driver complaints on a regular basis to track and report maintenance that needs to be performed by Ozark Trucking, because the trucks are leased. A summary of the complaints is shown in Table 11. The driver complaints are shown for before and after the data clean point (January 1 , 1998) for the LNG trucks. The diesel trucks have six months of complaints summarized.

The major complaints of the LNG trucks were problems with engine low power, running rough; body damage; lights out; fuel gauges; and $\mathrm{CB} / \mathrm{radio}$ /phone/antenna problems. The problems with the engine low power, running rough had the highest number of complaints, 51 percent before the clean point and 24 
percent after. Many of these complaints stemmed from the differences between operating an LNG truck and the diesel trucks. The LNG trucks were equipped with an L10-300G engine versus the diesels, which were equipped with M11-330s. The M11 had higher available torque (1250 ft-lb for the M11 vs. $900 \mathrm{ft}$ $\mathrm{lb}$ for the natural gas L10). Combined with the lower horsepower, this difference in torque was noticeable to the drivers.

Table 11. Number of Driver Complaints by Category

\begin{tabular}{|c|c|c|c|c|c|c|}
\hline \multirow[b]{2}{*}{ Complaint Category } & \multicolumn{2}{|c|}{ LNG (4/97-12/97) } & \multicolumn{2}{|c|}{ LNG (1/98-12/98) } & \multicolumn{2}{|c|}{ Diesel $(7 / 98-12 / 98)$} \\
\hline & $\begin{array}{l}\text { Number of } \\
\text { Complaints }\end{array}$ & $\begin{array}{l}\text { Percent of } \\
\text { Total (\%) }\end{array}$ & $\begin{array}{l}\text { Number of } \\
\text { Complaints }\end{array}$ & $\begin{array}{l}\text { Percent of } \\
\text { Total (\%) }\end{array}$ & $\begin{array}{l}\text { Number of } \\
\text { Complaints }\end{array}$ & $\begin{array}{l}\text { Percent of } \\
\text { Total (\%) }\end{array}$ \\
\hline Body Damage & 28 & 7 & 67 & 17 & 8 & 21 \\
\hline Lights & 42 & 10 & 40 & 10 & 10 & 26 \\
\hline Engine Low Power, Rough & 216 & 51 & 94 & 24 & 0 & 0 \\
\hline Low Fuel Pressure Light & 5 & 1 & 34 & 9 & 0 & 0 \\
\hline Fuel Leak & 25 & 6 & 15 & 4 & 0 & 0 \\
\hline Fuel Gauge & 32 & $\overline{8}$ & 29 & 7 & 0 & 0 \\
\hline Air/Suspension & 7 & 2 & 16 & 4 & 3 & 8 \\
\hline $\mathrm{AC} /$ Heat & 9 & 2 & 13 & 3 & 1 & 3 \\
\hline CB/Radio/Phone/Antenna & 23 & 5 & 27 & 7 & 3 & 8 \\
\hline Fifth Wheel & 6 & 1 & 2 & 1 & 0 & $\frac{0}{0}$ \\
\hline Cruise Control & 4 & 1 & 7 & 2 & 6 & 16 \\
\hline Wheels & 3 & 1 & 1 & 0 & 0 & 0 \\
\hline Clutch & 2 & 1 & 1 & 0 & 1 & 3 \\
\hline CADEC & 2 & 1 & 9 & 2 & 0 & $\overline{0}$ \\
\hline Starter/Alternator/Battery & 10 & 2 & 12 & 3 & 1 & 3 \\
\hline Tire & 1 & 0 & 16 & 4 & 3 & 8 \\
\hline Other & 5 & 1 & 10 & 3 & 2 & 4 \\
\hline Total & 420 & 100 & 393 & 100 & 38 & $\overline{100}$ \\
\hline Complaints per Truck & 53 & - & 49 & $\overline{-}$ & 13 & $\overline{-}$ \\
\hline
\end{tabular}

Also, early in the deployment of the LNG trucks, several part quality and hardiness issues arose. Under warranty, Cummins changed wastegate valves, several sensors, spark plugs and wires, and ignition modules on all of the trucks over the period of time that these issues were being investigated. As of January 1998, Cummins believed that these start up issues were resolved. As Figure 9 shows, the trend in complaints from drivers of the LNG trucks was consistent with the history of engine issues and their resolution in late December 1997. In Figure 9, each peak is in response to new drivers starting to use the LNG trucks. Within a month or two of a change in drivers, the number of complaints on the engine low power issue went up, peaked, and went back down. The number of complaints in 1998 for the engine low power issue went down dramatically. 


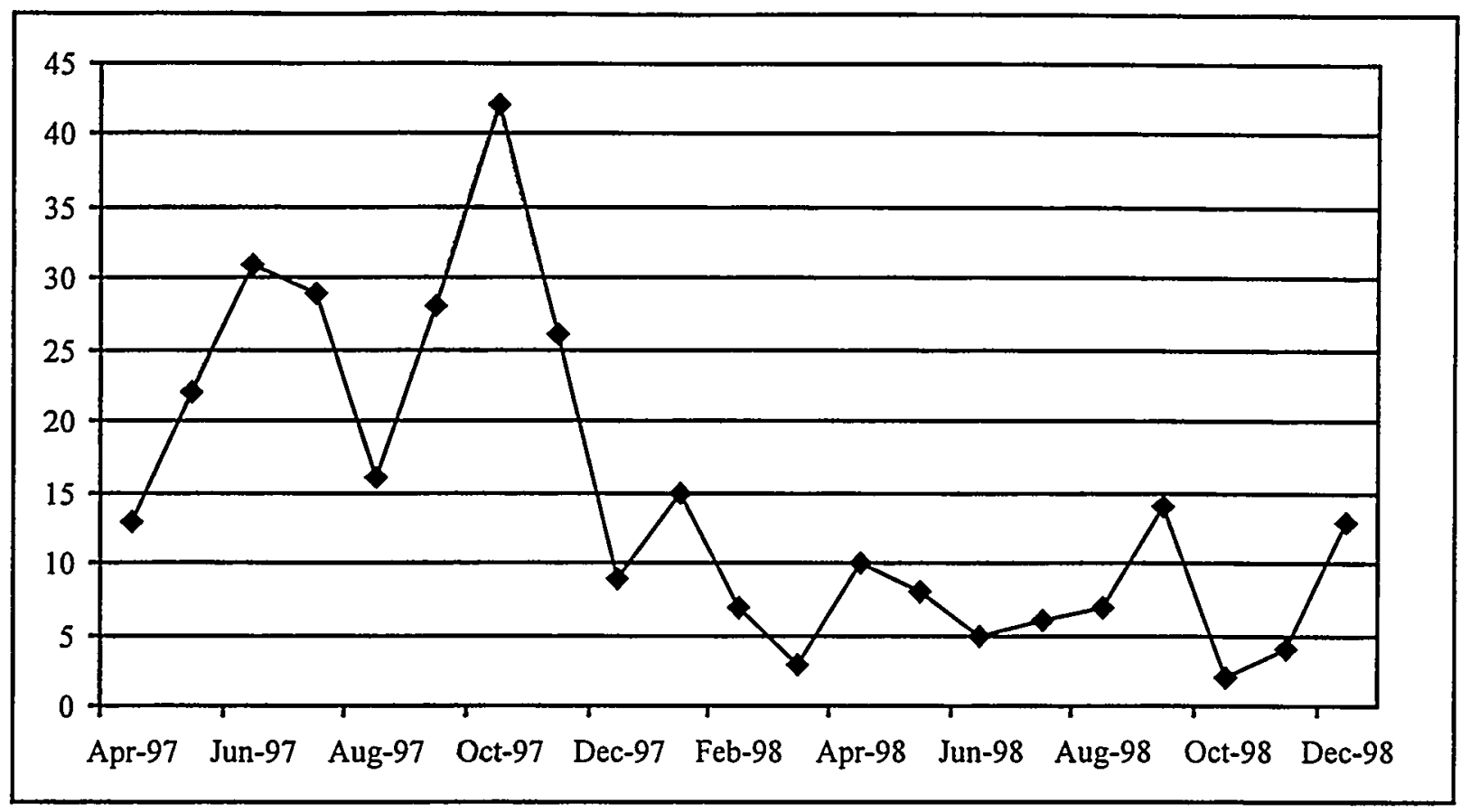

Figure 9. Number of Driver Complaints for Low Power and Rough Operation by Month for LNG Trucks

The complaints for the diesel trucks were mostly for lights, body damage, cruise control, tires, suspension, and $\mathrm{CB} / \mathrm{radio}$ /phone/antenna. These complaints are very similar to the LNG trucks except for the low power complaints.

\section{Warranty Repairs}

In regards to warranty repair actions, Table 12 shows the warranty items replaced by truck since the start of operation of the LNG trucks. As mentioned above, Cummins had problems with the wastegate, which has been modified for operation at Raley's. These engines were developed for the transit bus market and have operated successfully for several years. The ignition modules on the trucks had problems in their manufacture. These problems led to numerous spark plug, wire, and ignition coil replacements during the evaluation. Each of the LNG trucks had the HOS sensor replaced several times. Figure 10 shows the number of warranty claims from Cummins during the operation of the LNG trucks at Raley's. The curve shows a large number of claims in the early months of operation; however, as suggested by Cummins, most of the claims and problems had been resolved by January 1998. No warranty information was collected for the diesel trucks. 
Table 12. Cummins Warranty Engine Items Replaced by Truck

\begin{tabular}{|c|c|c|c|c|c|c|c|c|c|}
\hline Warranty Item Replaced & 1501 & 1502 & 1503 & 1504 & 1505 & 1506 & 1507 & 1508 & Total \\
\hline Sensor - HOS & 3 & 3 & 2 & 5 & 3 & 2 & 5 & 3 & 26 \\
\hline Sensor - PRS & 0 & 1 & 0 & 1 & 1 & $\overline{1}$ & 0 & 0 & 4 \\
\hline Sensor - Mass Flow & 0 & 0 & $\overline{0}$ & 1 & $\overline{1}$ & 0 & 1 & 0 & 3 \\
\hline Wastegate & 3 & 3 & 3 & 2 & 3 & 2 & 2 & 2 & 20 \\
\hline Spark Plugs & 18 & 12 & 12 & 19 & 14 & 6 & 23 & 12 & 116 \\
\hline Spark Plug Wires & 3 & 8 & 0 & 1 & 0 & 3 & 1 & 1 & 17 \\
\hline Ignition Module & 3 & 1 & 1 & 2 & 1 & 1 & 1 & 3 & 13 \\
\hline Ignition Coil & 0 & 0 & 0 & 2 & 0 & 0 & 2 & 2 & $\frac{7}{6}$ \\
\hline Governor & 1 & 1 & 2 & $\overline{0}$ & 0 & 0 & 0 & 0 & 4 \\
\hline Valve-Fuel Pressure & 0 & 1 & 2 & 2 & 0 & 0 & 3 & 0 & 8 \\
\hline Sensor - Fuel Pressure & 0 & 0 & 0 & 0 & 0 & 0 & 1 & 0 & 1 \\
\hline Valve - Fuel & 0 & 0 & 0 & 0 & 3 & 0 & 2 & 0 & 5 \\
\hline Actuator - Wastegate & 1 & 1 & 0 & 0 & 0 & 0 & 0 & 0 & 2 \\
\hline Turbocharger & 0 & 0 & 1 & 0 & 0 & 0 & 0 & 0 & 1 \\
\hline Throttle Pedal & 1 & 0 & 0 & 1 & 0 & 0 & 0 & 0 & 2 \\
\hline Air Compressor & 0 & 0 & 0 & 0 & 0 & 0 & 1 & 0 & 1 \\
\hline Total Warranty Cost (\$) & 11,903 & 11,538 & 12,561 & 13,981 & 8,104 & 6,951 & 13,829 & 6,577 & $\overline{85,444}$ \\
\hline
\end{tabular}

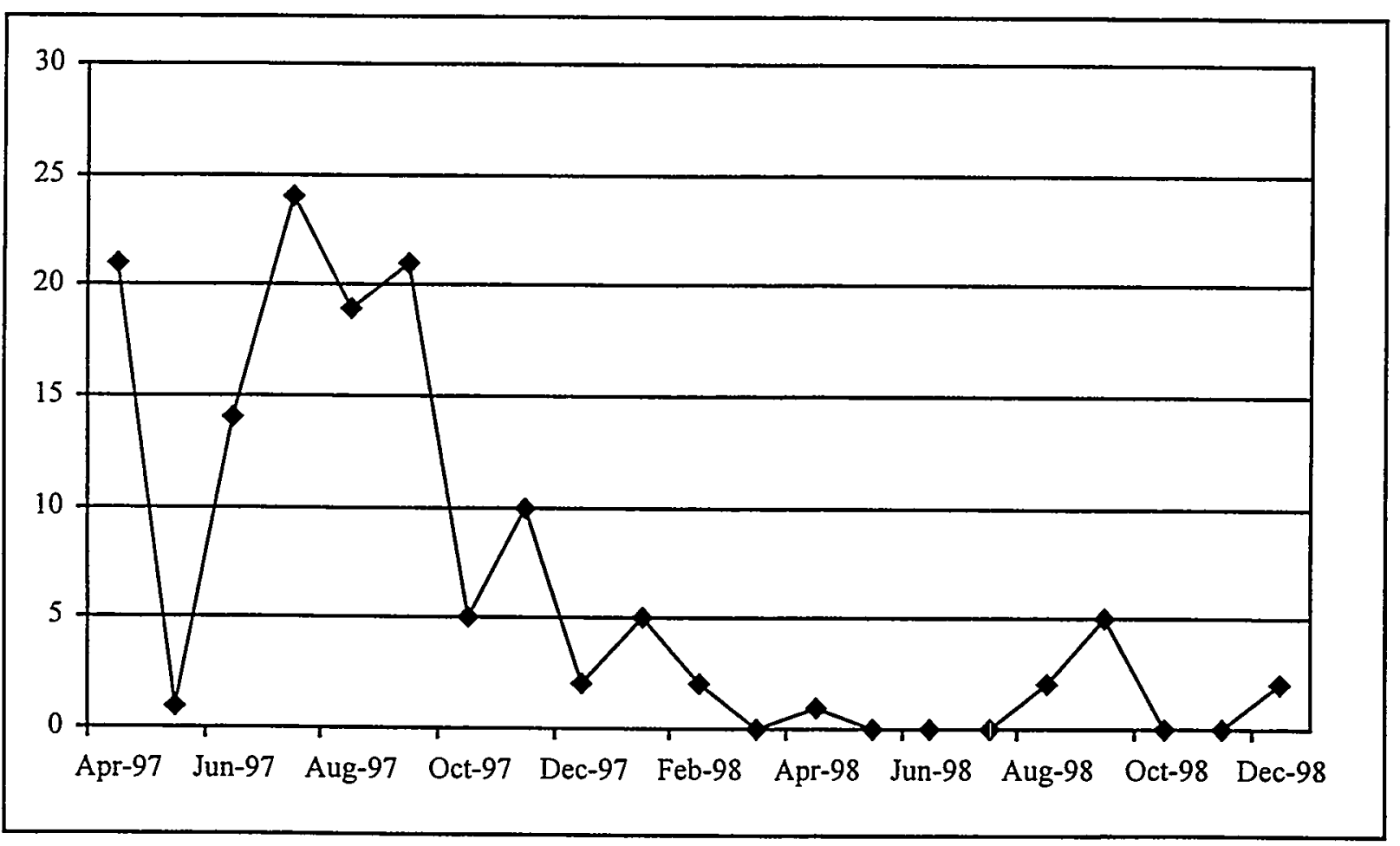

Figure 10. Number of Cummins Warranty Claims by Month for LNG Trucks 
As shown in Figures 9 and 10, the driver complaints and warranty repairs for the LNG trucks had a peak in September 1998. This was caused by problems with trucks 1501, 1503, and 1506. All three trucks were running badly (low power and back firing) and had the wastegate valve replaced along with other repair actions such as resetting calibration of the HOS sensor and ECM.

\section{Safety Incidents}

There were no safety incidents reported at Raley's or Ozark Trucking during the data collection. A few minor body damage incidents were reported, but none involved the LNG systems. 


\section{Summary of Operations Costs}

Table 13 provides a summary of the operations costs for the LNG and diesel trucks at Raley's without the driver labor cost. The fuel costs used are the average for the after clean point data period for diesel and the new LNG cost for the permanent LNG station at Raley's ( $\$ 1.01$ per gallon diesel and $\$ 1.09$ per diesel energy equivalent gallon for LNG). The maintenance costs do not include warranty repairs paid for by the manufacturers, and the labor cost is assumed to be $\$ 50$ per hour.

\section{Table 13. Summary of Operations Costs}

\begin{tabular}{|c|c|c|c|}
\hline Truck & $\begin{array}{c}\text { Fuel Usage Cost/Mile } \\
(\$)\end{array}$ & $\begin{array}{c}\text { Maintenance Cost/Mile } \\
(\$)\end{array}$ & $\begin{array}{c}\text { Total Cost/Mile } \\
(\$)\end{array}$ \\
\hline 1501 & 0.250 & 0.108 & 0.358 \\
\hline 1502 & 0.244 & 0.071 & 0.315 \\
\hline 1503 & 0.259 & 0.113 & 0.372 \\
\hline 1504 & 0.249 & 0.072 & 0.321 \\
\hline 1505 & 0.257 & 0.101 & 0.358 \\
\hline 1506 & 0.258 & 0.118 & 0.376 \\
\hline 1507 & 0.255 & 0.096 & 0.351 \\
\hline 1508 & 0.248 & 0.094 & 0.342 \\
\hline LNG & $\mathbf{0 . 2 5 2}$ & $\mathbf{0 . 0 9 6}$ & $\mathbf{0 . 3 4 8}$ \\
\hline 1586 & 0.144 & 0.042 & 0.186 \\
\hline 1592 & 0.145 & 0.038 & 0.183 \\
\hline 1593 & 0.143 & 0.061 & 0.204 \\
\hline Diesel & 0.144 & $\mathbf{0 . 0 4 8}$ & $\mathbf{0 . 1 9 2}$ \\
\hline
\end{tabular}




\section{Emissions Test Results}

One purpose in using alternative fuel vehicles is to reduce harmful emissions associated with traditional motor fuels such as gasoline and diesel fuel. As part of the evaluation of LNG and diesel fleets at Raley's, tests were conducted to compare the emissions of both types of trucks quantitatively.

The Department of Mechanical and Aerospace Engineering at West Virginia University (WVU) tested the emissions of the Raley's LNG and diesel control trucks soon after the LNG trucks went into service (July and August 1997) and then again in early 1998 (February and March 1998). Appendix H shows the detailed data reports from WVU for both emissions testing visits to Raley's.

\section{Emissions Testing Mobile Laboratory}

Under sponsorship of the U.S. Department of Energy (DOE), WVU designed and constructed a transportable chassis dynamometer to test for emissions levels from heavy-duty vehicles. The purpose of the transportable chassis dynamometer is to allow for a large number of "real-world" emissions tests to be performed on heavy-duty vehicles around the country. The first transportable unit was built in 1991, and WVU has been traveling to transit agencies and heavy-duty vehicle sites around the country testing buses and trucks since early 1992 . WVU built a second unit in 1994 and began testing vehicles with the second unit in 1995.

Emissions data are collected by WVU personnel using the mobile chassis dynamometer laboratory at least once for each participating site in this project. The WVU transportable laboratory can measure particulate matter $(\mathrm{PM})$, carbon monoxide $(\mathrm{CO})$, carbon dioxide $\left(\mathrm{CO}_{2}\right)$, nitrogen oxides $\left(\mathrm{NO}_{\mathrm{x}}\right)$, total hydrocarbons (HC), methane $\left(\mathrm{CH}_{4}\right)$, methanol $\left(\mathrm{CH}_{3} \mathrm{OH}\right)$, formaldehyde $(\mathrm{HCHO})$, and other emissions components. WVU uses a five peak, five-mile simulated route shown in Figure 11 to test each truck. The route is defined as a fixed distance route (five miles). The time the truck takes to finish the route may change depending on the truck's ability to accelerate. The length of the constant speed (cruise) portions of the route is adjusted to add or subtract time at constant speed based on the time taken for the truck to accelerate. All results from emissions testing are provided to the participating site as soon as the information is available. WVU strives to reduce any disruptions to normal operations at the host site during emissions testing.

\section{Emissions Test Results at Raley's}

Figure 12 shows the WVU laboratory set up at Raley's during the second round of testing. As shown in

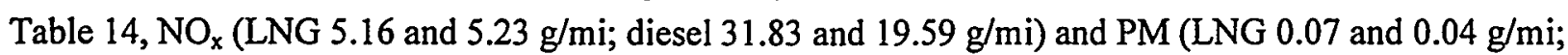
diesel 0.84 and $1.22 \mathrm{~g} / \mathrm{mi}$ ) are much lower for the LNG trucks. When comparing non-methane hydrocarbons (NMHC) for the LNG trucks and HC for the diesel control trucks (NMHC for the diesel trucks is essentially the same as HC results, very little methane), the results show the LNG trucks have 


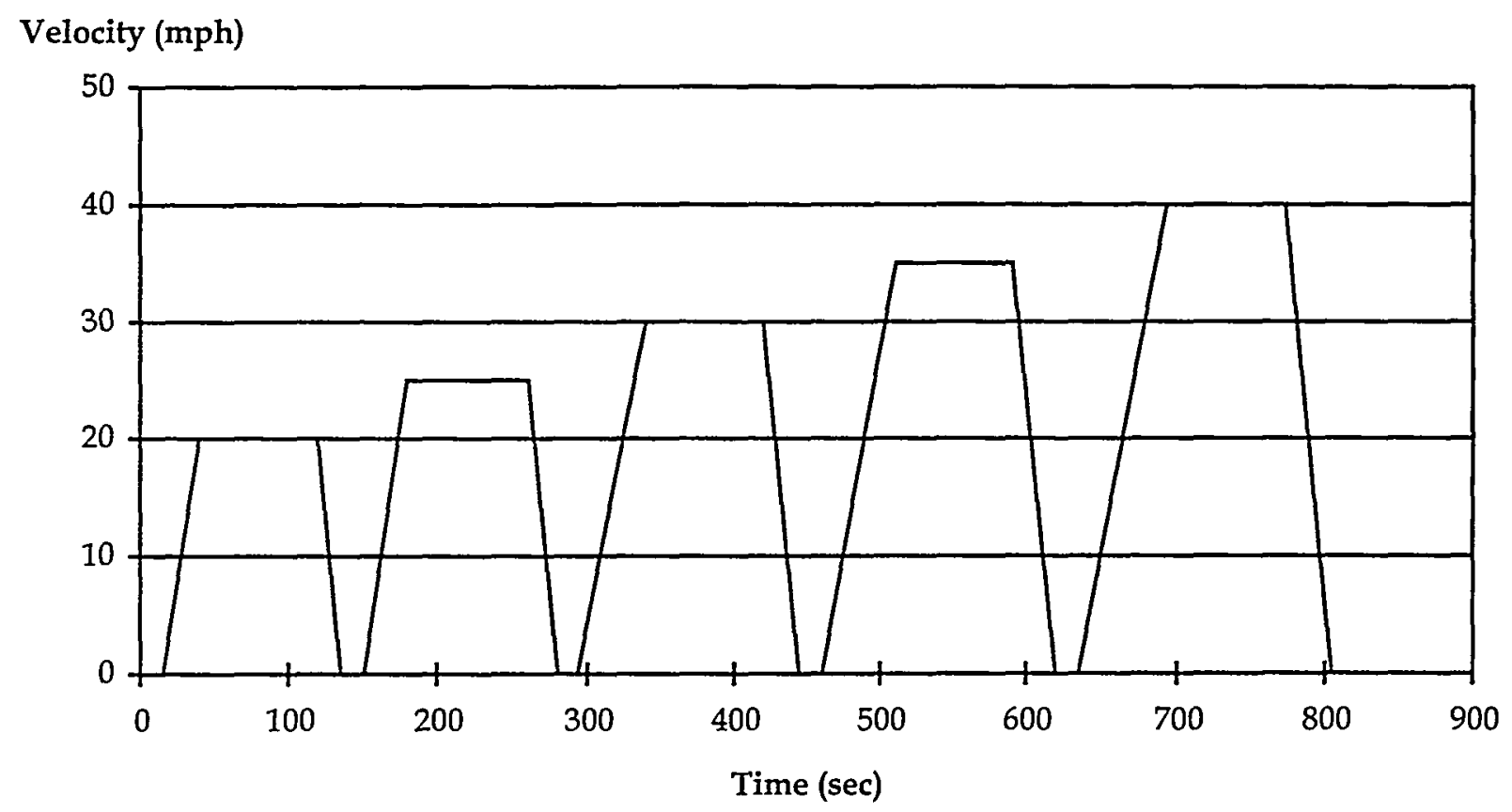

Figure 11. WVU Five-Peak Truck Cycle

Table 14. Summary of Emissions Testing Results

\begin{tabular}{|c|c|c|c|c|c|c|c|c|c|}
\hline $\begin{array}{l}\text { Test } \\
\text { Date }\end{array}$ & Fleet & Cycle & $\begin{array}{c}\mathrm{CO} \\
(\mathrm{g} / \mathrm{mi})\end{array}$ & $\begin{array}{c}\mathrm{NO}_{\mathrm{x}} \\
(\mathrm{g} / \mathrm{mi})\end{array}$ & $\underset{(\mathrm{g} / \mathrm{mi})}{\mathrm{HC}}$ & $\begin{array}{c}\text { NMHC } \\
(\mathrm{g} / \mathrm{mi})\end{array}$ & $\begin{array}{c}\mathbf{P M} \\
(\mathrm{g} / \mathrm{mi})\end{array}$ & $\begin{array}{c}\mathrm{CO}_{2} \\
(\mathrm{~g} / \mathrm{mi})\end{array}$ & MPEG \\
\hline \multirow{2}{*}{$7 / 97-8 / 97$} & LNG & 5-Mile Route & 7.12 & 5.16 & 17.51 & 0.60 & 0.07 & 1657 & 4.39 \\
\hline & Diesel & 5-Mile Route & 1.88 & 31.83 & 1.29 & 4he & 0.84 & 1620 & 6.26 \\
\hline \multirow{2}{*}{$2 / 98-3 / 98$} & LNG & 5-Mile Route & 7.92 & 5.25 & 21.37 & 0.62 & 0.04 & 1821 & 4.00 \\
\hline & Diesel & 5-Mile Route & 2.26 & 19.59 & 1.70 & 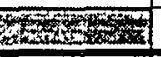 & 1.22 & 1658 & 6.11 \\
\hline $7 / 97-8 / 97$ & LNG/Diesel & 5-Mile Route & $279 \%$ & $-84 \%$ & (1) & $-53 \%$ & $-92 \%$ & $2 \%$ & $-30 \%$ \\
\hline $2 / 98-3 / 98$ & LNG/Diesel & 5-Mile Route & $250 \%$ & $-73 \%$ & 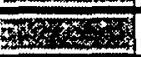 & $-64 \%$ & $-97 \%$ & $10 \%$ & $-35 \%$ \\
\hline
\end{tabular}

LNG/Diesel data are calculated by the following equation $-($ LNG/Diesel -1$) * 100$

MPEG - miles per energy equivalent diesel gallon 



Figure 12. WVU Dynamometer Laboratory at Raley's

significantly lower results (LNG 0.60 and $0.62 \mathrm{~g} / \mathrm{mi} \mathrm{NMHC;} \mathrm{diesel} 1.29$ and $1.70 \mathrm{~g} / \mathrm{mi} \mathrm{HC}$ ). The LNG trucks have significantly higher $\mathrm{CO}$ results and modestly higher $\mathrm{CO}_{2}$ results. The miles per diesel energy equivalent gallon (MPEG) shown in the table is essentially the same in respect to the difference between the diesel and LNG energy equivalent results as compared to the in-use fuel economy data.

Note that some differences in the LNG truck results have occurred between the two testing visits. This is most likely related to the changes to the engine configuration and some of the components that were 
upgraded as part of the warranty repairs. For the two diesel testing results, the $\mathrm{NO}_{\mathrm{x}}$ results are significantly lower for the second testing visit. This lower $\mathrm{NO}_{\mathrm{x}}$ result is most likely an issue of engine map (calibration) and which gears were used during the drive cycle. The continuous $\mathrm{NO}_{\mathrm{x}}$ data suggests that an off-cycle injection timing strategy was at play for the diesel vehicles.

For reference, Table 15 shows the emissions certification standards for the model years and actual results for the LNG and diesel engines used in this study. The newer Cummins L10 natural gas engine emissions certification testing results are also shown. The numbers in Table 15 do not relate directly to the emissions results from the WVU testing. The results in Table 15 have been compiled from engine certification testing and the results from the WVU testing were compiled from chassis dynamometer testing.

\section{Table 15. Heavy Engine Emissions Certification Standards and Certification Data for Raley's Trucks (grams per brake-horsepower hour)}

\begin{tabular}{|l|c|c|c|c|c|}
\hline \multicolumn{1}{|c|}{ Standard/Certification } & NMHC (HC) & CO & NOx & PM & EPA Engine Family Name \\
\hline 1994-1997 EPA/CARB Truck & $1.2(1.3)$ & 15.5 & 5.0 & 0.10 & \\
\hline 1998 EPA/CARB Truck & $1.2(1.3)$ & 15.5 & 4.0 & 0.10 & \\
\hline Truck L10-300G - 1997 & & & & & \\
With Catalyst & 0.5 & 5.3 & 2.4 & 0.03 & VCE611FBCABW \\
Without Catalyst & 1.1 & 11.2 & 3.5 & 0.06 & VCE611EBCARW \\
\hline Truck L10-300G - 1998 & 0.1 & 4.6 & 1.6 & 0.02 & WCEXH0611LAB \\
With Catalyst & 0.8 & 10.6 & 2.5 & 0.07 & WCEXH0611LAC \\
Without Catalyst & $(0.2)$ & 1.4 & 4.2 & 0.07 & TCE661EJDASW \\
\hline Truck M11-330 - 1996 &
\end{tabular}

Raley's LNG engines are not equipped with a catalyst, but have the same engine calibration as the 1998 certification without catalyst

Raley's diesel control trucks are model year 1996. 


\section{Conclusions}

The major conclusions from the evaluation of the Raley's LNG truck site include the following:

- The LNG trucks have been used as planned. The LNG trucks have been used for local Sacramento area pickup and delivery at the request of the Sacramento Metropolitan Air Quality Management District in return for funding to help establish LNG operations.

- Drivers perceive the LNG trucks to be slightly under-powered for Raley's operation.

- The emissions results from the Raley's project show large decreases in NOx and PM for the LNG trucks. The NOx reduction for the eight LNG trucks using the WVU testing results would have a nearly 7 tons per year reduction compared to diesel trucks running in place of the LNG trucks.

- Energy equivalent fuel economies between in-use fuel data and emissions testing fuel economies are essentially consistent. The energy equivalent fuel economy comparison between LNG and diesel trucks at Raley's is about 38 percent lower for the LNG trucks. This has caused some problems with range for the LNG trucks. This lower fuel economy has been aggravated by having fuel gauges that are not reliable.

- Mileage per vehicle per calendar month and year was significantly lower for the LNG trucks compared to the diesel control trucks. This lower mileage for the LNG trucks was caused by how the trucks have been used (routes closer to the Distribution Center in the Sacramento area) as well as problems with lower range than the diesel trucks and engine problems experienced early in the data collection period. The lower vehicle mileage for the LNG trucks has caused the cost per mile for maintenance to be biased higher than expected because the preventive maintenance actions are performed on a quarterly basis, not a mileage basis. To better understand how the LNG trucks have performed, the maintenance cost analysis has been made on a cost per truck basis for the same length of data period to account for this bias. Maintenance costs per mile are also provided.

- Operations and LNG fuel costs during the evaluation were affected by a change from a temporary to a permanent LNG fueling and storage station at Raley's. There were several major delays in starting and completing the construction of the permanent LNG station. The permanent LNG station allows Raley's to purchase a full tanker truckload of LNG, thus reducing the cost by $\$ 0.10$ per LNG gallon.

- Availability of LNG in the local Sacramento, California area could reduce LNG fuel cost significantly by reducing transportation costs (currently coming from Wyoming or Arizona). 
- Raley's staff indicated that their operation would benefit from a larger LNG storage tank at the permanent fueling station (currently 13,000 gallons). The tank has reserve for vapor and liquid that cannot be used, which reduces the useable volume to about 11,500 gallons. This leaves a margin for error of 1,500 gallons when ordering fuel. Fuel shipments must be scheduled carefully, or the station with either run out of fuel or will not be able to accommodate the full incoming shipment, which results in lost fuel that Raley's must pay for.

- There were some problems with the LNG engines, such as with the wastegate, ignition modules, and sensor calibration. The LNG engine manufacturer, Cummins, was supportive and responsive to these problems. Operations were fairly trouble-free at the end of the evaluation.

- Raley's intends to expand their LNG truck operations; however, there are few choices of heavyduty natural gas truck technologies.

- As part of this project, Raley's purchased two LNG yard tractors. These vehicles have been extremely successful for Raley's. The drivers have noted that, compared to the older diesel yard tractors, the LNG yard tractors offer reduced noise and vibration as well as a lack of diesel fumes. 


\section{Status of Site in Project}

Data collection and evaluation for this project were completed with data from December 1998. As of the end of December 1998, the new LNG station was online. Raley's planned to return the QRS refueling station to Cummins West. Raley's had planned to allow public access to their LNG station to help other fleets get started using LNG and to help offset the fuel station cost. However, public access to the new LNG station was waiting for approval from Weights and Measures as of the writing of this report. There was a discrepancy in how to measure LNG fuel accurately for dispensing fuel for sale to other fleets.

Raley's was looking at options to expand their LNG fleet. The Cummins L10-300G engine was discontinued for new LNG purchases because of low sales volume and because the L10 was discontinued for diesel as well. Raley's is currently in discussions with Cummins West to possibly be involved with a research project to test one or two prototype $400 \mathrm{hp}$ engines. Other potential technology options to expand the fleet in the future may be Caterpillar Dual-Fuel (C10 or C12), Mack (E7), Detroit Diesel (Series 60), and Westport high-pressure direct injection technology.

\section{Other Project-Related Reports}

Battelle, Raley's LNG Truck Fleet, Start-Up Experience, National Renewable Energy Laboratory, Golden, CO, 1997, NREL/BR-540-23402.

Battelle, Dual-Fuel Truck Fleet, Start-Up Experience, National Renewable Energy Laboratory, Golden, CO, 1998, NREL/BR-540-25118.

Chandler, K., Norton, P., Clark, N., 1999, Interim Results from Alternative Fuel Truck Evaluation Project, 1999, SAE International, Warrendale, PA, SAE Pub. \#1999-01-1505.

Chandler, K., Norton, P., Clark, N., Alternative Fuel Truck Evaluation Project-Design and Preliminary Results, 1998, 981392, SAE International, Warrendale, PA.

Clark, N., Lyons, D., Rapp, B., Gautam, M., Wang, W., Norton, P., White, C., Chandler, K., Emissions from Trucks and Buses Powered by Cummins L-10 Natural Gas Engines, 1998, 981393, SAE International, Warrendale, PA.

Project Guidelines, Alternative Fuel Truck Evaluation Project, 1998, Battelle, Columbus, Ohio. 


\section{Acknowledgements}

This project would not be possible without the help and cooperation of the staff at the host sites. The authors gratefully acknowledge the Raley's office staff at the Distribution Center for their support in providing data on a timely basis. Also, the authors acknowledge Ed Gamache at Ozark Trucking and Stan Sasaki at Raley's for providing input to the data collection, analyses, and reports. 


\section{Contacts}

\section{Stan Sasaki}

Manager of Transportation

Raley's Distribution Center

4061 Gateway Park Boulevard

Sacramento, California 95834

Phone: (916) 928-7747

Fax: (916) 928-0761

Kevin Chandler

Project Manager

Battelle

505 King Avenue

Columbus, Ohio 43201

Phone: (614) 424-5127

Fax: (614) 424-5069

e-mail: chandlek@battelle.org

\section{Paul Norton}

Senior Project Engineer

National Renewable Energy Laboratory

1617 Cole Boulevard

Golden, Colorado 80401

Phone: (303) 275-4424

Fax: (303) 275-4415

e-mail: Paul_Norton@nrel.gov

\section{Ed Gamache}

Fleet Maintenance Manager

Ozark Trucking Inc.

900 E Street

West Sacramento, California 95605

Phone: (916) 372-1472

Fax: (916) 371-2119

\section{Vinod Duggal}

Director

Advanced Engineering, Alternate Fuels

Cummins Engine Company, Inc.

1900 McKinley Avenue

Columbus, Indiana 47201

Phone: (812) 377-7338

Fax: (812) 377-2029 
Appendix A

Map of Raley's Locations and Listing of Locations 


\section{All Raley's Store Locations}

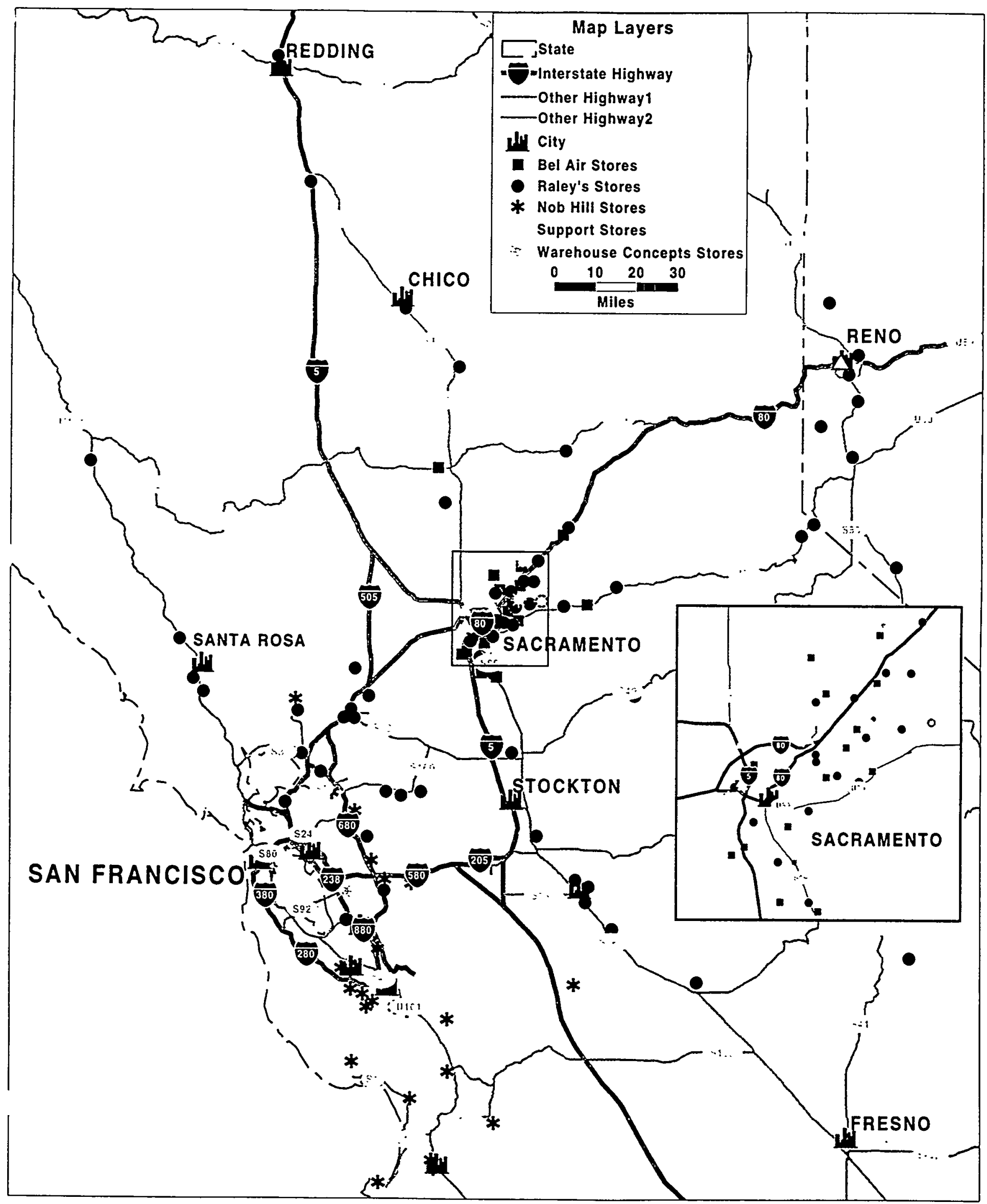




\section{Nob Hill Stores}

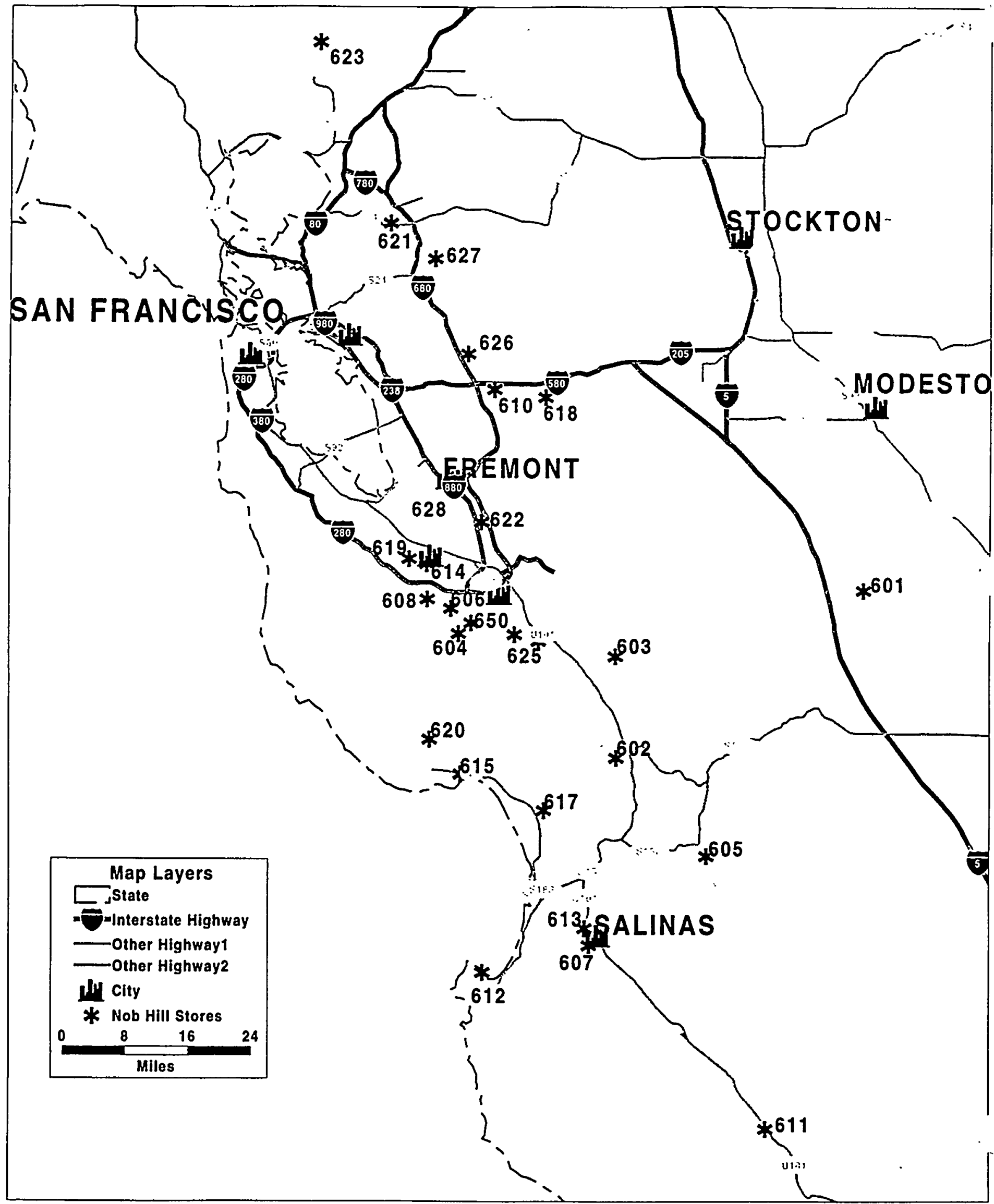




\section{Bel Air Stores}

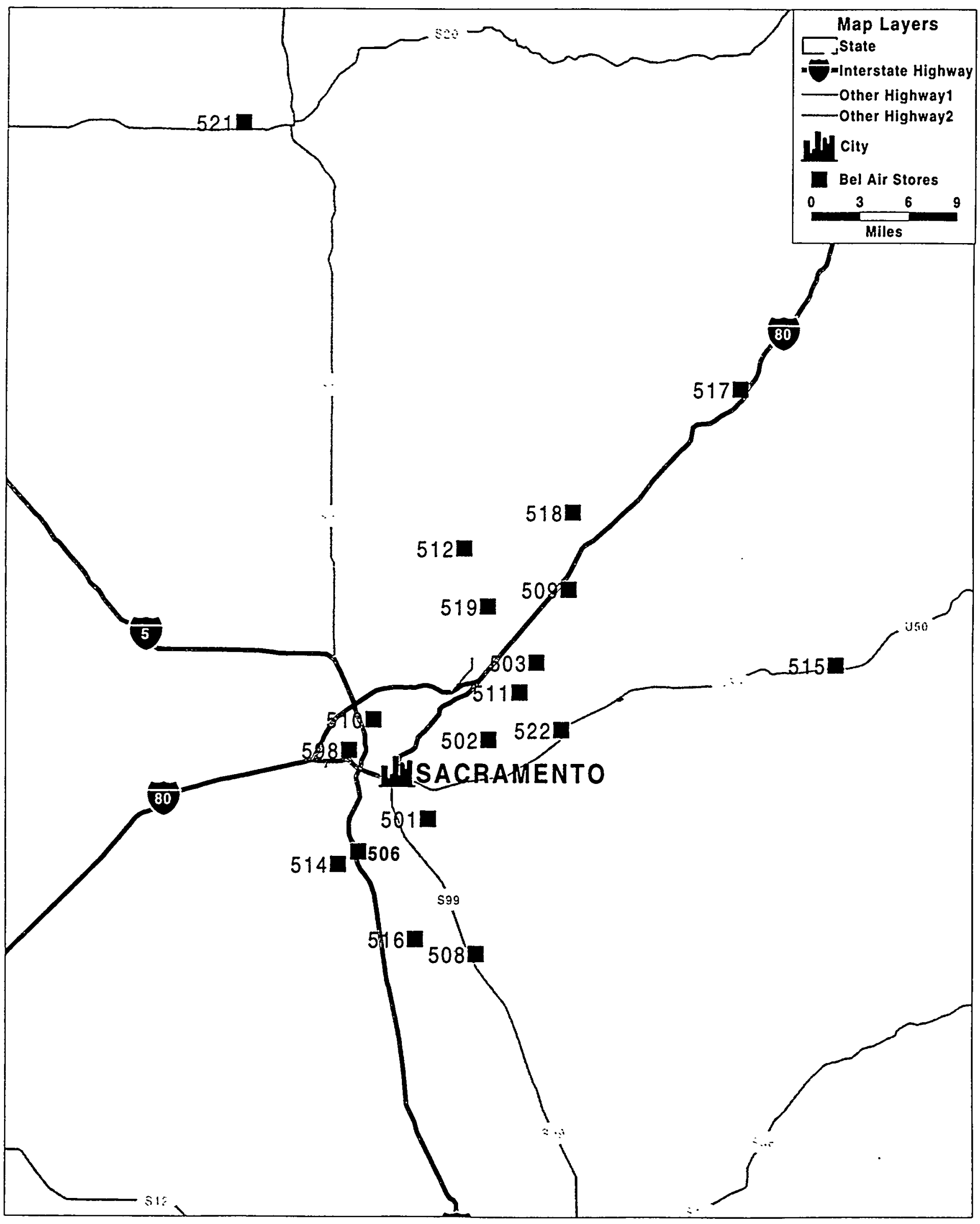




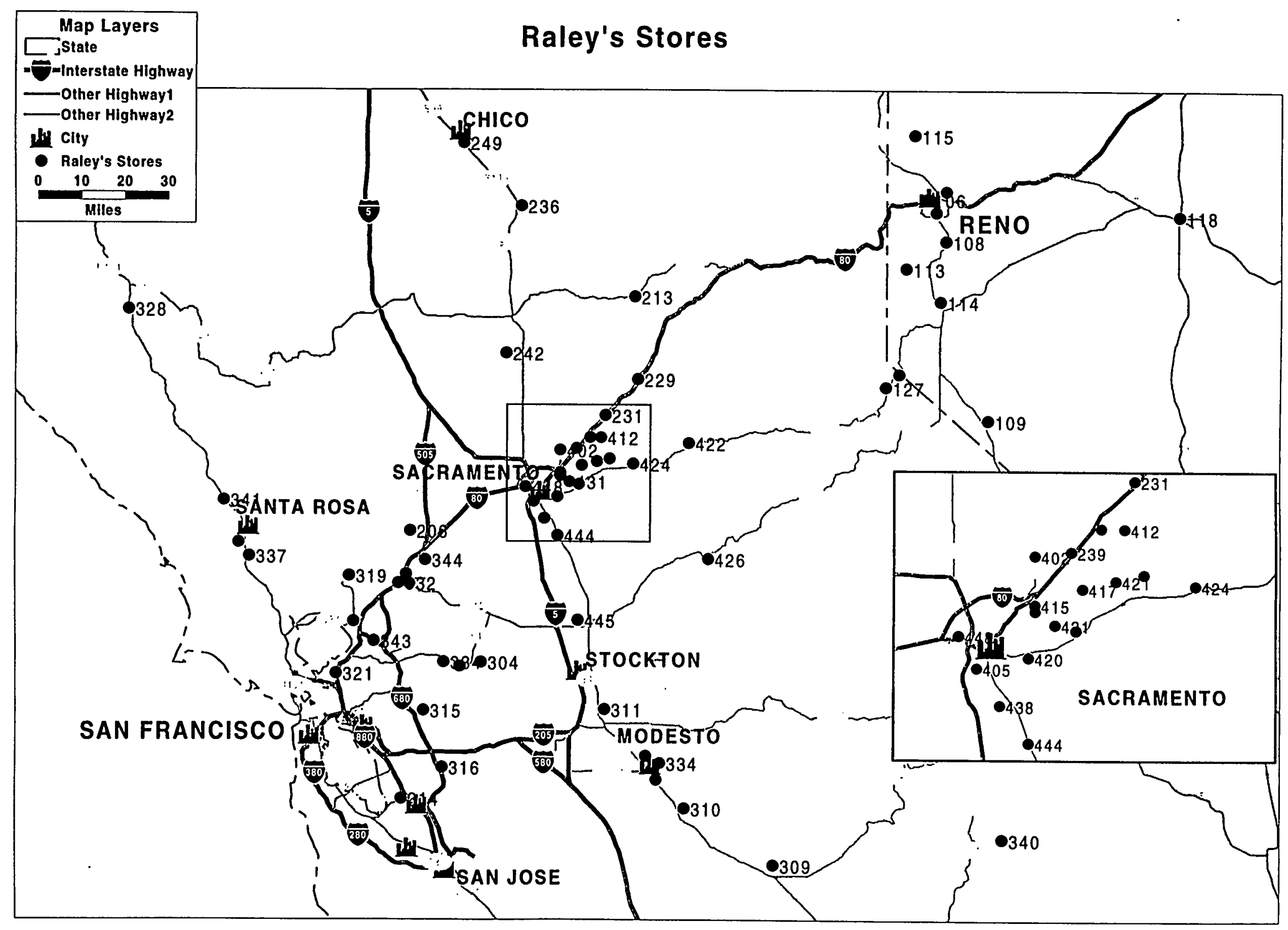

$\because \leftrightarrow m, \ldots$ 


\section{Raley's Support Stores}

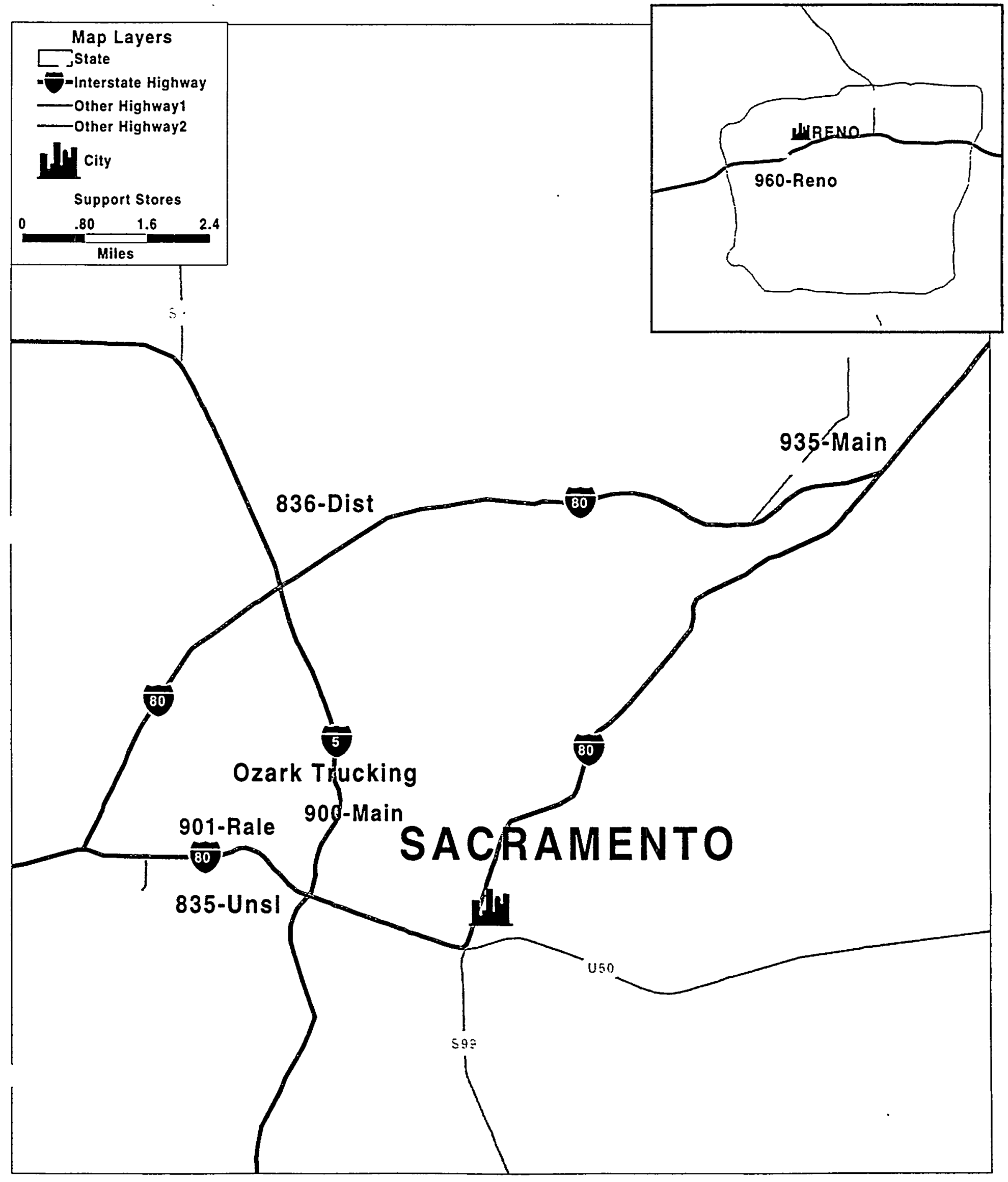




\section{Warehouse Concepts Stores}

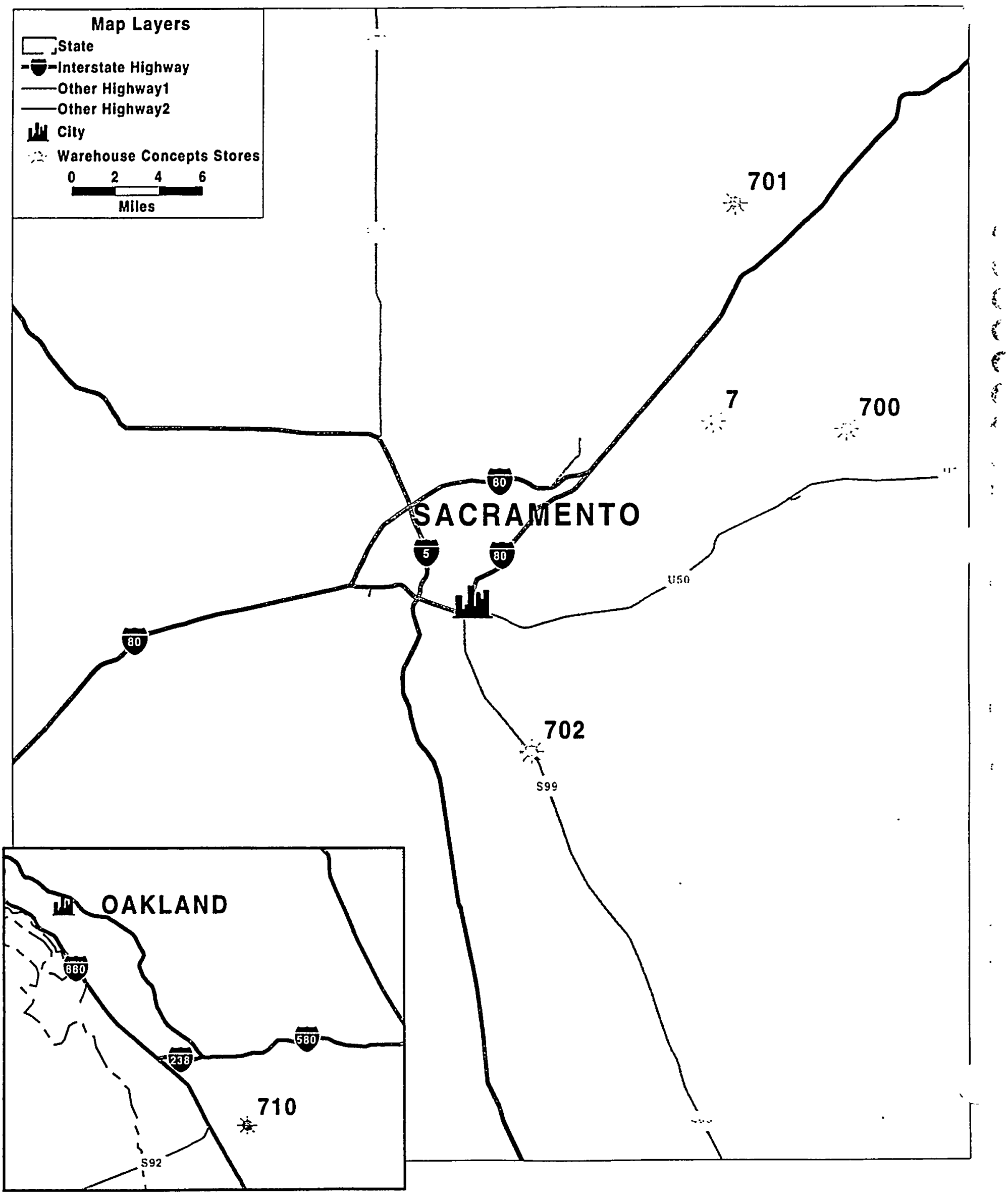




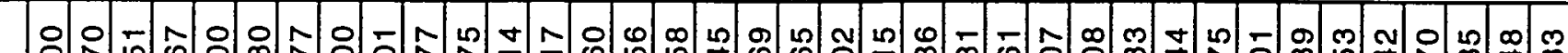

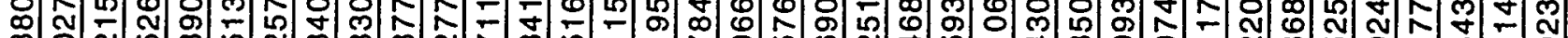

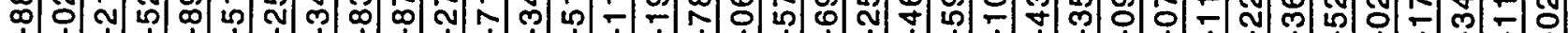

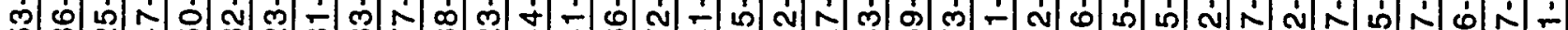
¿ б

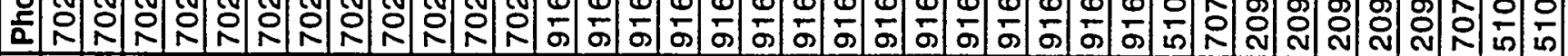

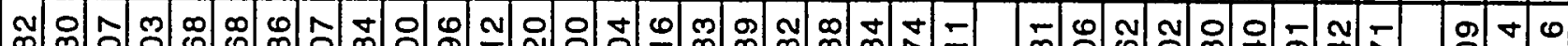
ఐ \% N - $\sim$ 壬앤유

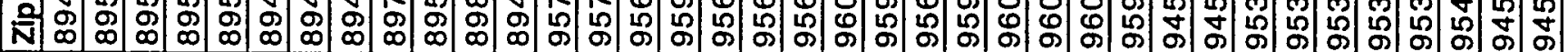
$\underset{\mathbf{D}}{\mathbf{D}}$

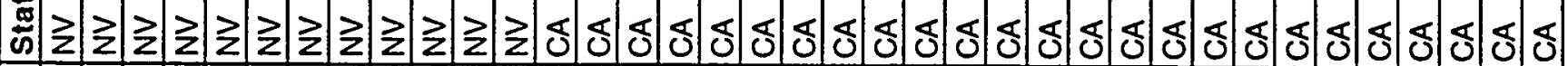

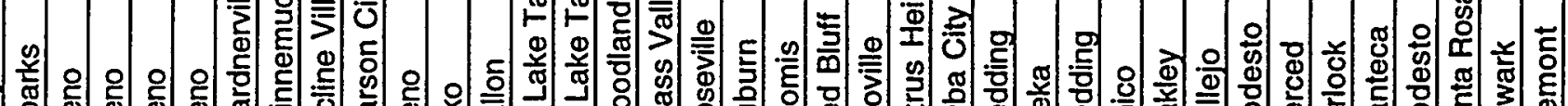
궁

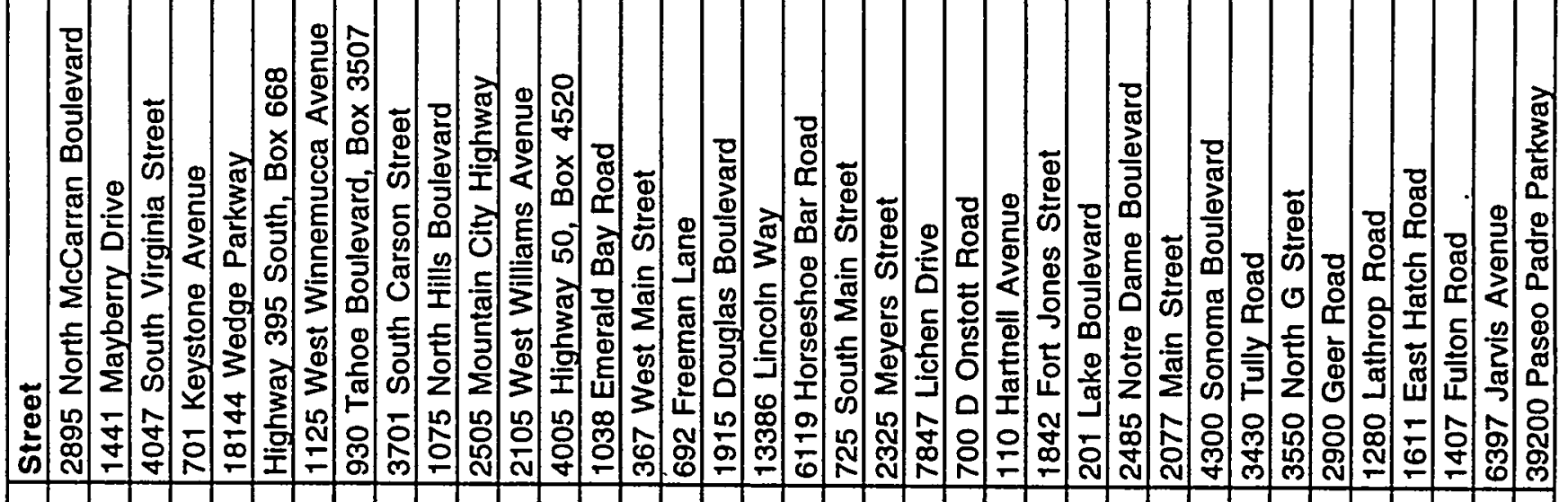

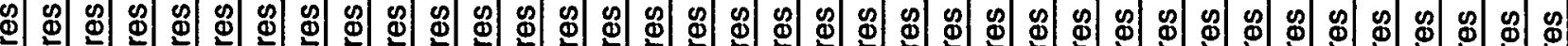

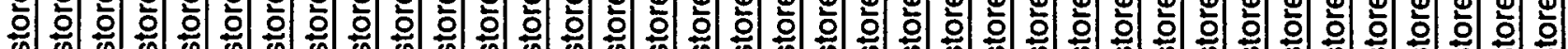

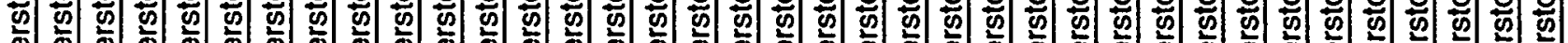

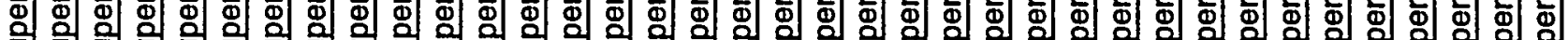

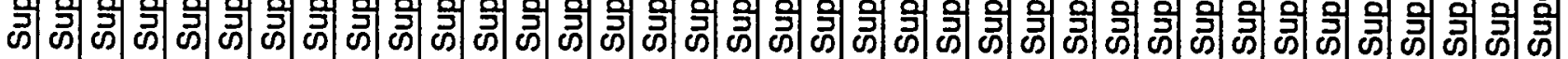

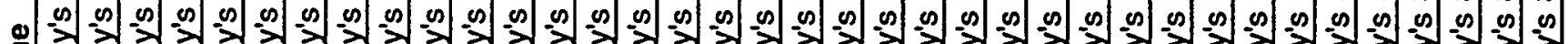

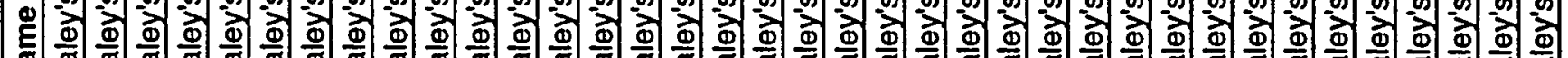

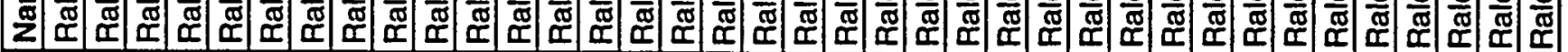

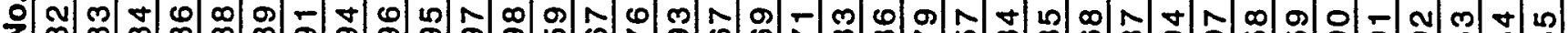

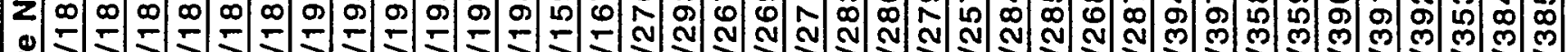

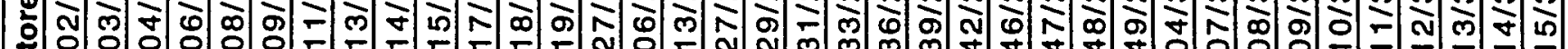

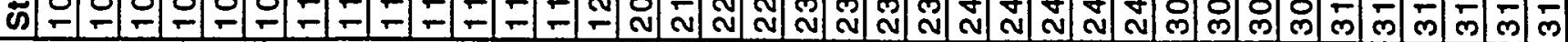




\begin{tabular}{|c|c|c|c|c|c|c|}
\hline Store No. & Name & Street & City & State & Zip & Phone \\
\hline $316 / 386$ & Raley's Superstores & 5420 Sunol Boulevard, Suite 1 & Pleasanton & $\mathrm{CA}$ & $94566-7701$ & $510-846-4471$ \\
\hline $319 / 369$ & Raley's Superstores & 217 Soscol Avenue & Napa & $\mathrm{CA}$ & $94559-4007$ & $707-224-7897$ \\
\hline $321 / 351$ & Raley's Superstores & 3360 San Pablo Dam Road & San Pablo & $\mathrm{CA}$ & $94806-2724$ & $510-222-5935$ \\
\hline $328 / 388$ & Raley's Superstores & 1315 North State Street & Ukiah & CA & $95482-3419$ & $707-468-5178$ \\
\hline $330 / 380$ & Raley's Superstores & 270 Sunset Avenue & Suisun City & $\mathrm{CA}$ & $94585-1766$ & $707-426-1023$ \\
\hline $331 / 371$ & Raley's Superstores & 3330 North Texas Street & Fairfield & $\mathrm{CA}$ & $94533-9715$ & $707-421-9990$ \\
\hline $332 / 372$ & Raley's Superstores & 3001 Travis Boulevard & Fairfield & CA & $94533-3442$ & $707-429-8410$ \\
\hline $333 / 373$ & Raley's Superstores & 3632 Lone Tree Way & Antioch & CA & $94509-6001$ & $510-754-2522$ \\
\hline $334 / 374$ & Raley's Superstores & 2401 East Orangeburg Avenue & Modesto & CA & $95355-3351$ & $209-577-5656$ \\
\hline $335 / 375$ & Raley's Superstores & 1369 Buchanan Road & Pittsburg & $\mathrm{CA}$ & $94565-6406$ & $510-432-1900$ \\
\hline $337 / 377$ & Raley's Superstores & 100 Raley's Town Center & Rohnert Park & $\mathrm{CA}$ & $94928-2440$ & $707-585-3222$ \\
\hline $340 / 350$ & Aaley's Superstores & 40041 Highway 41, Box 367 & Oakhurst & $\mathrm{CA}$ & 93644-0367 & 209-683-8300 \\
\hline $341 / 356$ & Raley's Superstores & 8852 Lakewood Drive & Windsor & $\mathrm{CA}$ & $95492-9595$ & $707-838-6604$ \\
\hline $343 / 381$ & Paley's Superstores & 890 Southampton Road & Benicia & $\mathrm{CA}$ & $94510-1907$ & $707-746-1203$ \\
\hline $344 / 382$ & Raley's Superstores & 3063 Alamo Drive & Vacaville & $\mathrm{CA}$ & $95687-6394$ & $707-446-8707$ \\
\hline $402 / 452$ & Raley's Superstores & 7477 Watt Avenue & No. Highlands & CA & $95660-2606$ & $916-332-2635$ \\
\hline $405 / 453$ & Raley's Superstores & 4850 Freeport Boulevard & Sacramento & $\mathrm{CA}$ & $95822-2401$ & $916-452-6861$ \\
\hline $409 / 489$ & Raley's Superstores & 715 East Bidwell Street & Folsom & CA & $95630-3341$ & $916-983-7267$ \\
\hline $412 / 492$ & Raley's Superstores & 6847 Douglas Boulevard & Roseville & $\mathrm{CA}$ & $95661-8528$ & $916-791-8000$ \\
\hline 415 & Raley's Superstores & 3431 Watt Avenue & Sacramento & $\mathrm{CA}$ & $95821-3612$ & 916-483-0424 \\
\hline $416 / 456$ & Raley's Superstores & 3518 Marconi Avenue & Sacramento & CA & $95821-5302$ & $916-972-7177$ \\
\hline $417 / 454$ & Raley's Superstores & 4830 San Juan Avenue & Fair Oaks & $\mathrm{CA}$ & $95628-4719$ & $916-967-4718$ \\
\hline $418 / 455$ & Raley's Superstores & 10385 Folsom Boulevard & Rancho Cordova & $\mathrm{CA}$ & $95670-3518$ & $916-363-2691$ \\
\hline $420 / 460$ & Raley's Superstores & 8391 Folsom Boulevard & Sacramento & $\mathrm{CA}$ & $95826-3538$ & $916-383-6622$ \\
\hline $421 / 461$ & Raley's Superstores & 8870 Madison Avenue & Fair Oaks & $\mathrm{CA}$ & $95628-3908$ & $916-966-2201$ \\
\hline $422 / 462$ & Raley's Superstores & 160 Placerville Drive & Placerville & $\mathrm{CA}$ & $95667-3919$ & $916-622-6800$ \\
\hline $424 / 464$ & Raley's Superstores & 3935 Park Drive & El Dorado Hills & $\mathrm{CA}$ & $95762-4561$ & $916.933-0518$ \\
\hline $426 / 466$ & Raley's Superstores & 781 South Highway 49 & Jackson & CA & 95642 & $209-223-9650$ \\
\hline $431 / 471$ & Raley's Superstores & 5159 Fair Oaks Boulevard & Carmicheal & $\mathrm{CA}$ & $95608-5750$ & $916-483-9033$ \\
\hline $438 / 478$ & Raley's Superstores & 4551 Mack Road & Sacramento & CA & $95823-4532$ & $916-421-4523$ \\
\hline $444 / 484$ & Raley's Superstores & 9149 East Stockton Boulevard & Elk Grove & $\mathrm{CA}$ & $95624-9504$ & $916-685-1200$ \\
\hline $445 / 483$ & Raley's Superstores & 311 Lower Sacramento Road & Lodi & CA & $95240-2167$ & $209-369-1917$ \\
\hline $448 / 486$ & Raley's Superstores & 1601 West Capitol Avenue & West Sacramento & $\mathrm{CA}$ & $95691-3219$ & $916-372-3000$ \\
\hline 501 & Bel Air Markets & 6231 Fruitridge Road & Sacramento & CA & $95820-5814$ & 916-739-8647 \\
\hline 502 & Bel Air Markets & 4320 Arden Way & Sacramento & $\mathrm{CA}$ & $95864-3103$ & $916-972-0555$ \\
\hline 503 & Bel Air Markets & 5450 Dewey Drive & Fair Oaks & $\mathrm{CA}$ & $95628-3138$ & $916-967-4766$ \\
\hline 506 & Bel Air Markets & 1301 Florin Road & Sacramento & CA & $95831-3618$ & $916-421-6766$ \\
\hline
\end{tabular}


น

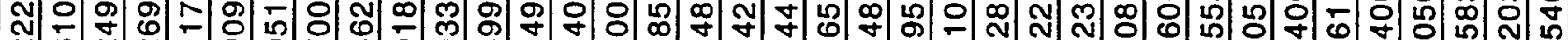
N 20

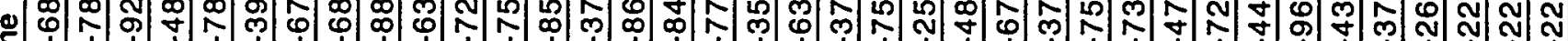
인

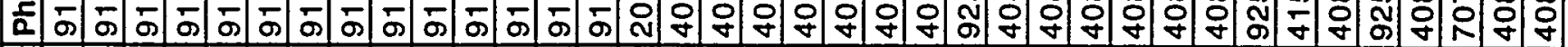

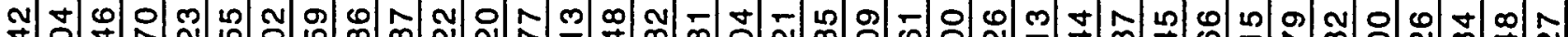

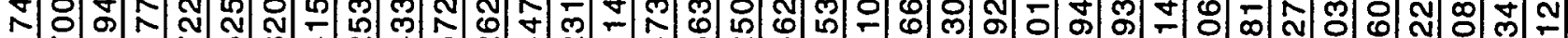

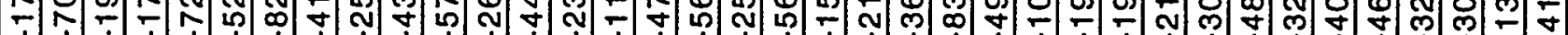

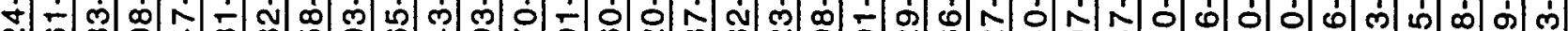
N

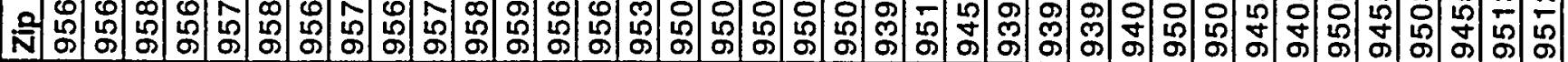
$\stackrel{9}{\mathbf{a}}$

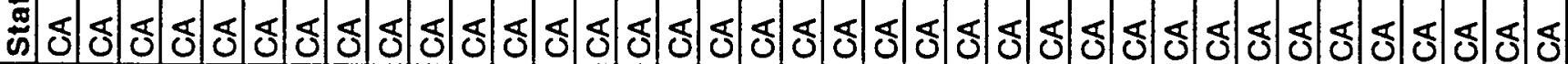

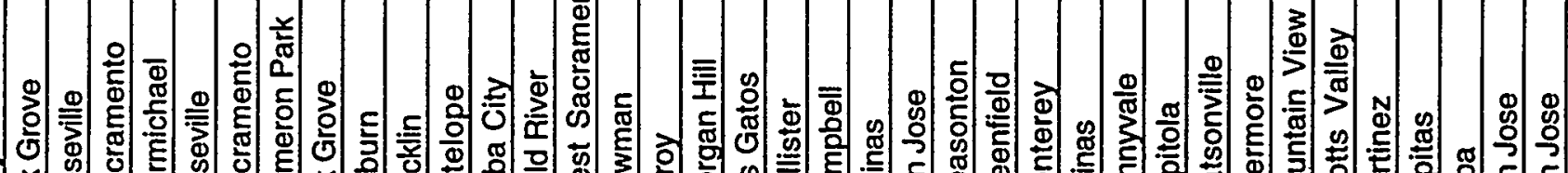

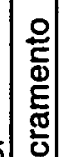

$\stackrel{\circ}{\frac{\mathrm{c}}{\mathrm{a}}}$

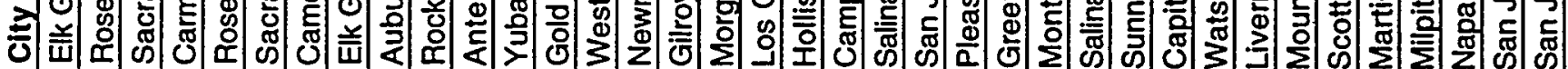

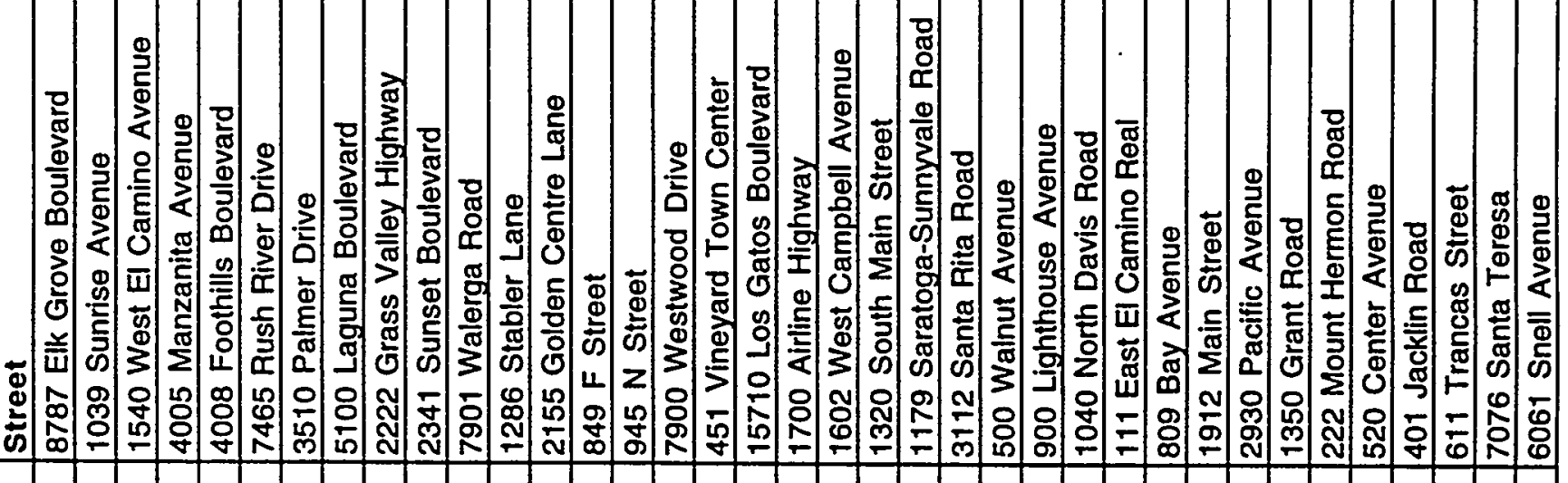

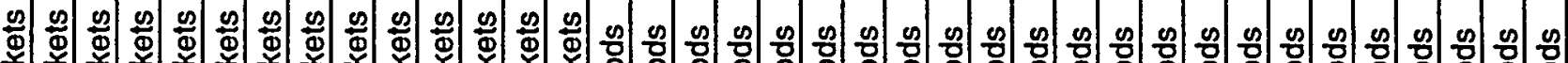

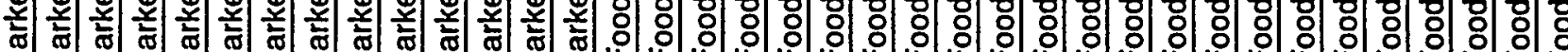

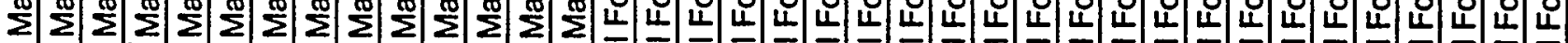

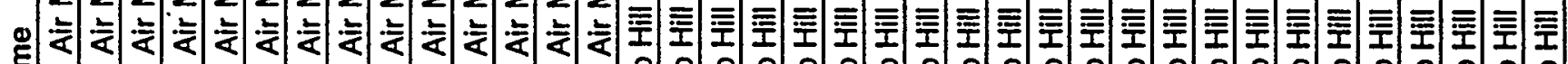

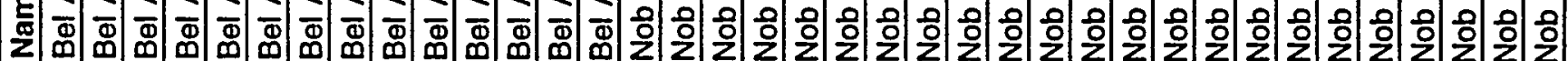

울

里

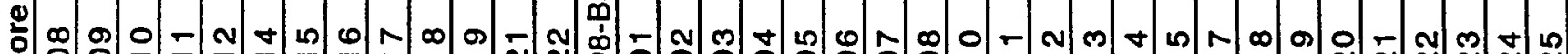

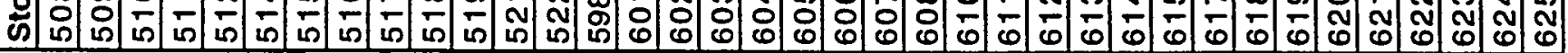




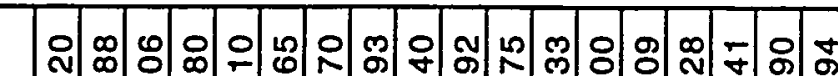

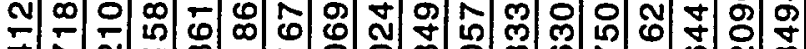
Y ๓ \& N б. ம்

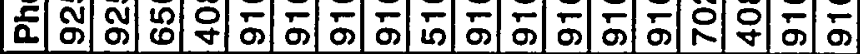
ㅇำ

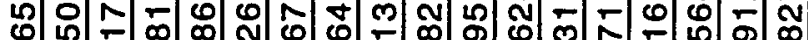
Nิ Nิ

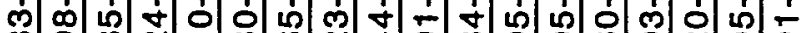
$\infty$ a N $\infty$ N

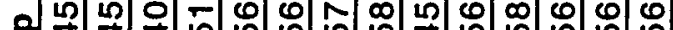

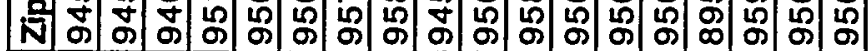
Q

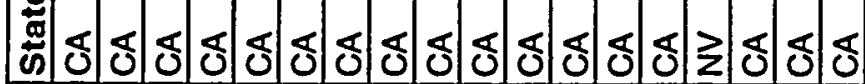

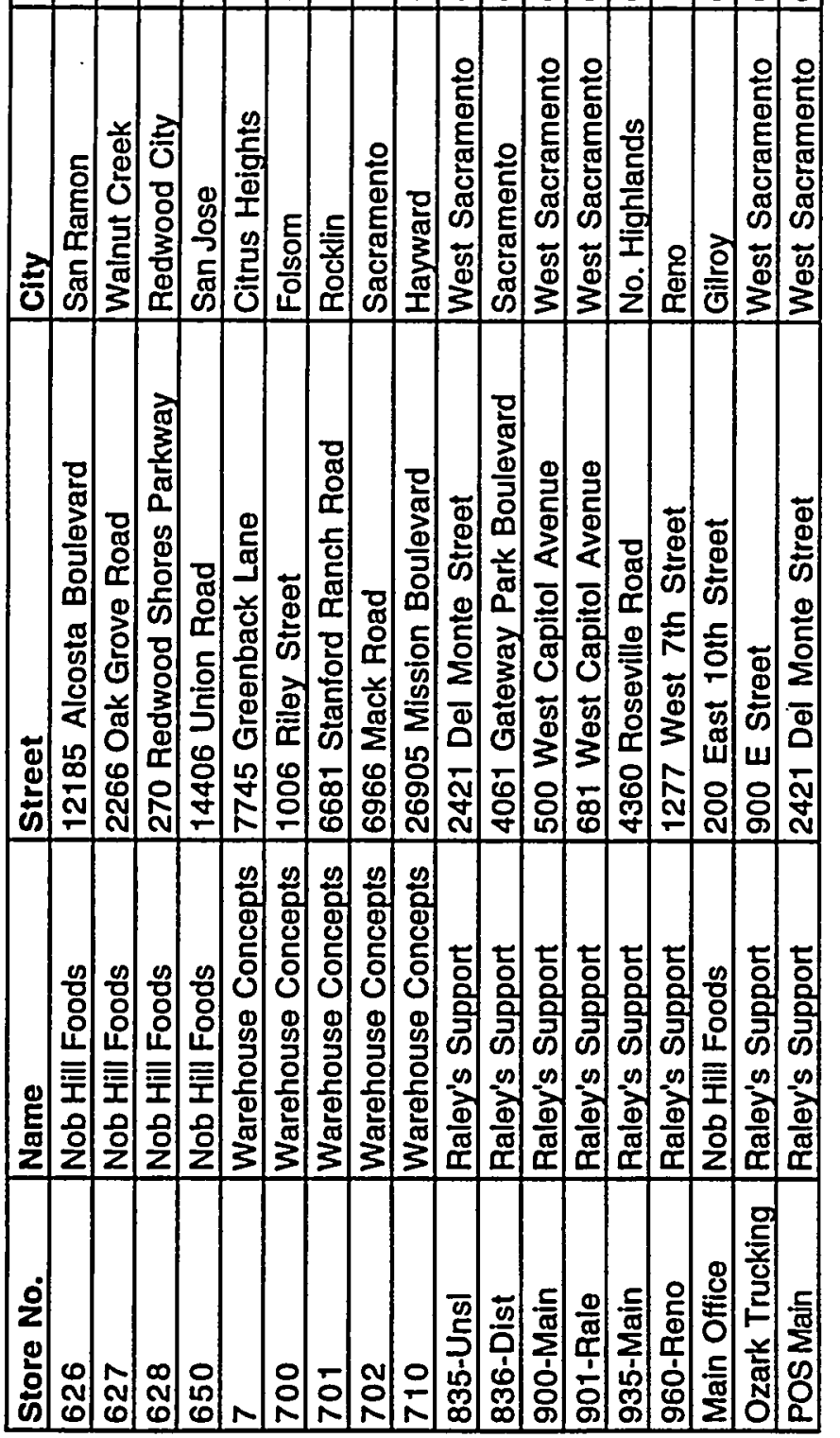


Appendix B

\section{Vehicle Descriptions}


Raley's LNG and Diesel Control Trucks

\begin{tabular}{|l|l|l|l|}
\hline Vehicle ID & \multicolumn{1}{|c|}{ Decal ID } & \multicolumn{1}{|c|}{ VIN } & ESN \\
\hline 1501 & RA001LKC & 1XKDD59X0VR745373 & 34845353 \\
\hline 1502 & RA002LKC & 1XKDD59X2VR745374 & 34847352 \\
\hline 1503 & RA003LKC & 1XKDD59X4VR745375 & 34847353 \\
\hline 1504 & RA004LKC & 1XKDD59X6VR745376 & 34847019 \\
\hline 1505 & RA005LKC & 1XKDD59X8VR745377 & 34849168 \\
\hline 1506 & RA006LKC & 1XKDD59XXVR745378 & 34849166 \\
\hline 1507 & RA007LKC & 1XKDD59X1VR745379 & 34849167 \\
\hline 1508 & RA008LKC & 1XKDD59X8VR745380 & 34849165 \\
\hline 1586 & RA009DKCC & 1XKBD99X9TS690099 & 34776206 \\
\hline 1592 & RA010DKCC & 1XKBD99X2TS712783 & 34786945 \\
\hline 1593 & RA011DKCC & 1XKBD99X4TS712784 & 34787997 \\
\hline
\end{tabular}




\begin{tabular}{|c|c|c|}
\hline Requested Information & Description & Site Data \\
\hline \multicolumn{3}{|c|}{ Vehicle Description } \\
\hline Owner/Operator & Name of organization that owns and/or operates vehicle & Ozark Trucking/Raley's \\
\hline Decal ID & A unique alphanumeric code for a vehicle used in this program & Shown in separate table \\
\hline Vehicle ID Number (VIN) & Vehicle Identification Number & Shown in separate table \\
\hline Fleet Vehicle ID & Vehicle Identification Number used by fleet & $1586,1592,1593$ \\
\hline Vehicle Make & Name of vehicle manufacturer & Kenworth \\
\hline Vehicle Model & Truck model number & T400 \\
\hline Vehicle Year & Year vehicle was manufactured & 1996 \\
\hline Service Date & Date vehicle was put into service by fleet & $10 / 1 / 95$ \\
\hline Start Mileage & Mileage on vehicle at the start of the fleet demonstration & 0 \\
\hline Activity Code & Type of activity vehicle is used for (Code 1 from VMRSH) & 12 (line-haul, refridgerated) \\
\hline Equipment Category Code & Type of optional equipment installed on vehicle & \\
\hline Body Manufacturer Code & Name of body manufacturer & \\
\hline Body Description Code & Type of body attached to cab (Code 48 from VMRSH) & \\
\hline Engine Serial Number & Serial number of the engine & Shown in separate table \\
\hline New Vehicle or Conv. Cost & Cost for each new vehicle or the conversion cost & $\$ 72,000$ \\
\hline \multicolumn{3}{|c|}{ Engine } \\
\hline OEM Retrofit & Is the engine OEM or a retrofit? & OEM \\
\hline Engine Manufacturer Code & Name of engine manufacturer & Cummins Engine $\mathrm{Co}$. \\
\hline Engine Model & Engine model number & M11-330 \\
\hline Engine Configuration Code & Engine Configuration Code (Code 35 from VMRSH) & 12 \\
\hline Engine Cubic Inches & Engine size in cubic inches & 661 \\
\hline
\end{tabular}


Vehicle System Descriptions (continued)

\begin{tabular}{|c|c|c|}
\hline Requested Information & Description & Site Data \\
\hline Engine Year & Year engine was manufactured & 1996 \\
\hline Cycle & Is the engine 2 cycle or 4 cycle? & 4 \\
\hline Compression Ratio & Compression ratio & 16.1:1 \\
\hline Ignition Aid Type & Type of ignition aids used & None \\
\hline Maximum bHp & Rated maximum brake horsepower of engine & 330 \\
\hline $\mathrm{RPM}$ of Max bHp & RPM at rated maximum brake horsepower & 1600 \\
\hline Maximum Torque (ft-lbs) & Rated maximum torque of engine & 1250 \\
\hline RPM of Max Torque & RPM at rated maximum torque & 1200 \\
\hline EPA Engine Family Name & Code assigned to engine by EPA for certification tracking & TCE661EJDASW \\
\hline \multicolumn{3}{|c|}{$\therefore \quad$ and System } \\
\hline Fuel Type Code & What type of fuel is the engine designed for? & Diesel \\
\hline Diesel Additives & Type of additives used in diesel fuel & None \\
\hline Alt Fuel Additives & Type of additives used in alternative fuel & N/A \\
\hline Mechanical or Electric & $\begin{array}{l}\text { For liquid fuel engines, are the injectors mechanically or } \\
\text { electronically controlled }\end{array}$ & Electronic \\
\hline Injector Manufacturer & Name of liquid fuel injector manufacturer & Cummins \\
\hline Injector Model & Liquid fuel injector model number & Celect \\
\hline
\end{tabular}


Vehicle System Descriptions (continued)

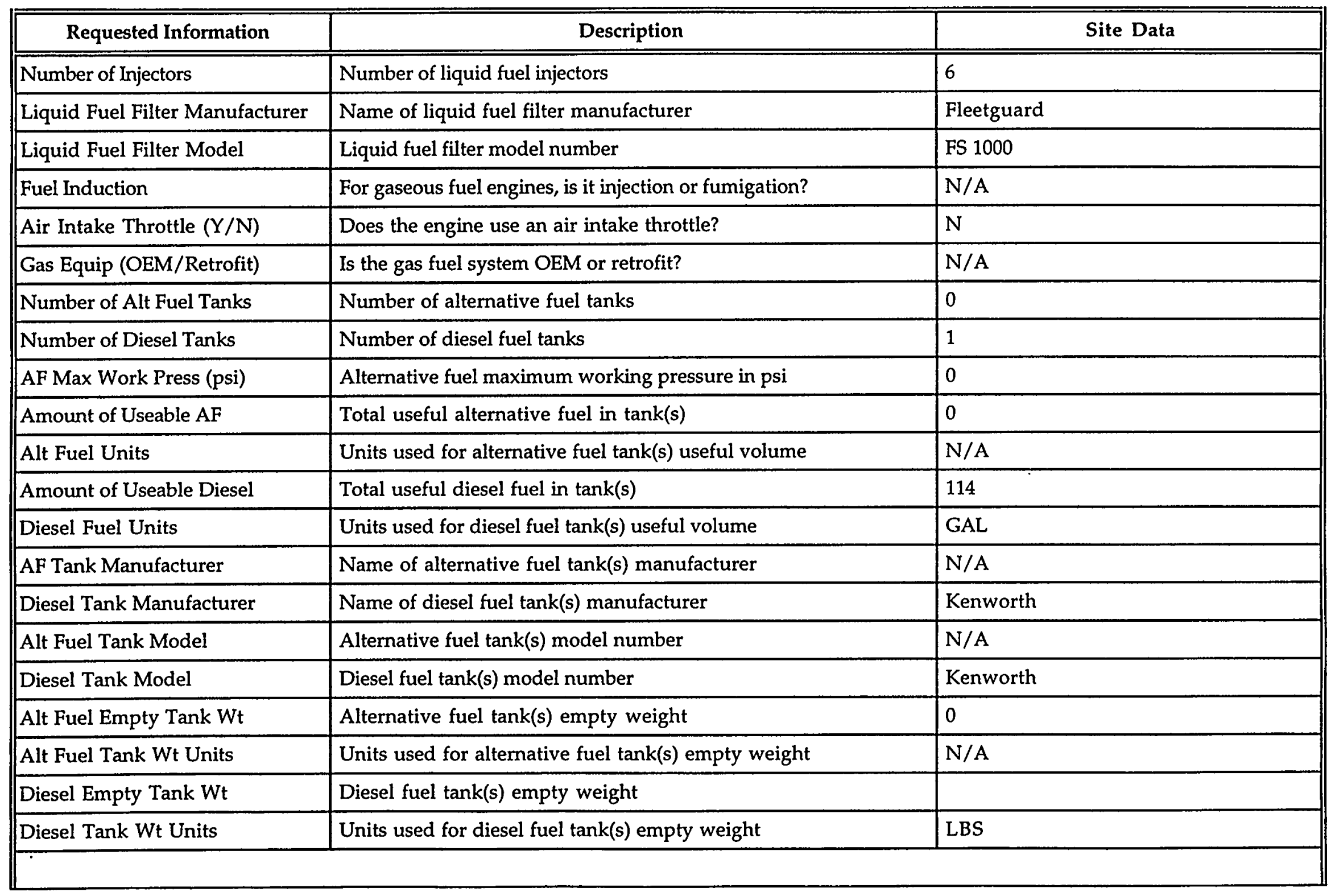


Vehicle System Descriptions (continued)

\begin{tabular}{|c|c|c|}
\hline Requested Information & Description & Site Data \\
\hline \multicolumn{3}{|c|}{ - } \\
\hline Transmission Manufacturer & Name of transmission manufacturer & Fuller \\
\hline Transmission Model Number & Transmission model number & RTL012610B \\
\hline Transmission Year of Mfr & Transmission year of manufacture & 1996 \\
\hline Transmission Type Code & Type of Transmission (Code 7 from VMRSH) & 1 \\
\hline Forward Speeds & Number of forward speeds & 10 \\
\hline Reverse Speeds & Number of reverse speeds & 2 \\
\hline Retarder $(Y / N)$ & Is the truck equipped with a retarder/Jake brake & Y \\
\hline 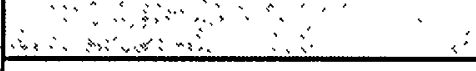 & 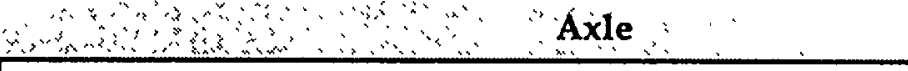 & , \\
\hline Axle Type Code & Type of axle configuration (Code 3 from VMRSH) & $\mathrm{D}$ \\
\hline Axle Front Weight & Axle front weight & 12,000 LBS \\
\hline Front Tire Size & Size of front tires & 295/75R22.5 \\
\hline Rear Tire Size & Size of rear tires & $295 / 75 R 22.5$ \\
\hline Axle Manufacturer Code & Name of drive axle manufacturer (from VMRSH) & Eaton \\
\hline Axle Model & Drive axle model number & DS 402 \\
\hline Rear Axle Config Code & Rear axle configuration (Code 37 from VMRSH) & \\
\hline Rear Axle Setup Code & Setup of rear axle configuration (Code 38 from VMRSH) & 2 \\
\hline Axle Ratio Low & Low axle ratio & 4.33:1 \\
\hline Axle Ratio High & High axle ratio & \\
\hline Total GVW Wt (lb) & Total gross vehicle weight in pounds & 80,000 LBS \\
\hline Total Curb Wt (lb) & Total weight with the truck in curb weight configuration & 14,440 LBS \\
\hline
\end{tabular}


Vehicle System Descriptions (continued)

\begin{tabular}{||l|l|l||}
\hline \multicolumn{1}{|c|}{ Requested Information } & \multicolumn{1}{|c|}{ Description } & \multicolumn{1}{|c|}{ Site Data } \\
\hline \hline Torque Converter Ratio & Torque converter ratio & N/A \\
\hline Wheelbase & Length of wheelbase & N \\
\hline Catalytic Converter & Does the vehicle have a catalytic converter? & \\
\hline Catalytic Converter Mfr & Name of catalytic converter manufacturer & N \\
\hline Catalytic Converter Model & Model number of the catalytic converter & \\
\hline Diesel Particulate Trap & Does the vehicle have a diesel particulate trap? & \\
\hline Trap Manufacturer & Name of the particulate trap manufacturer & \\
\hline Trap Model & Model number of the particulate trap & \\
\hline Trap Regeneration Type & Type of trap regeneration process & \\
\hline Trap Configuration & Particulate trap configuration & \\
\hline Number of Trap Elements & Number of particulate trap elements & \\
\hline Trap System Weight & Weight of the particulate trap system & \\
\hline
\end{tabular}

VMRSH - Vehicle Maintenance Reporting Standards Handbook from the American Trucking Associations and published by Management Systems Council. 
LNG

Vehicle System Descriptions

\begin{tabular}{|c|c|c|}
\hline Requested Information & Description & Site Data \\
\hline \multicolumn{3}{|c|}{ A veshicle Description } \\
\hline Owner/Operator & Name of organization that owns and/or operates vehicle & Ozark Trucking/Raley's \\
\hline Decal ID & A unique alphanumeric code for a vehicle used in this program & Shown in separate table \\
\hline Vehicle ID Number (VIN) & Vehicle Identification Number & Shown in separate table \\
\hline Fleet Vehicle ID & Vehicle Identification Number used by fleet & $1501-1508$ \\
\hline Vehicle Make & Name of vehicle manufacturer & Kenworth \\
\hline Vehicle Model & Truck model number & T800 \\
\hline Vehicle Year & Year vehicle was manufactured & 1997 \\
\hline Service Date & Date vehicle was put into service by fleet & $4 / 9 / 97$ \\
\hline Start Mileage & Mileage on vehicle at the start of the fleet demonstration & 0 \\
\hline Activity Code & Type of activity vehicle is used for (Code 1 from VMRSH) & 12 (line-haul, refridgerated) \\
\hline Equipment Category Code & Type of optional equipment installed on vehicle & \\
\hline Body Manufacturer Code & Name of body manufacturer & \\
\hline Body Description Code & Type of body attached to cab (Code 48 from VMRSH) & \\
\hline Engine Serial Number & Serial number of the engine & Shown in separate table \\
\hline New Vehicle or Conv. Cost & Cost for each new vehicle or the conversion cost & $\$ 107,816$ \\
\hline . & Engine & \\
\hline OEM Retrofit & Is the engine OEM or a retrofit? & OEM \\
\hline Engine Manufacturer Code & Name of engine manufacturer & Cummins Engine Co. \\
\hline Engine Model & Engine model number & L10-300G \\
\hline Engine Configuration Code & Engine Configuration Code (Code 35 from VMRSH) & 12 \\
\hline Engine Cubic Inches & Engine size in cubic inches & 611 \\
\hline
\end{tabular}


Vehicle System Descriptions (continued)

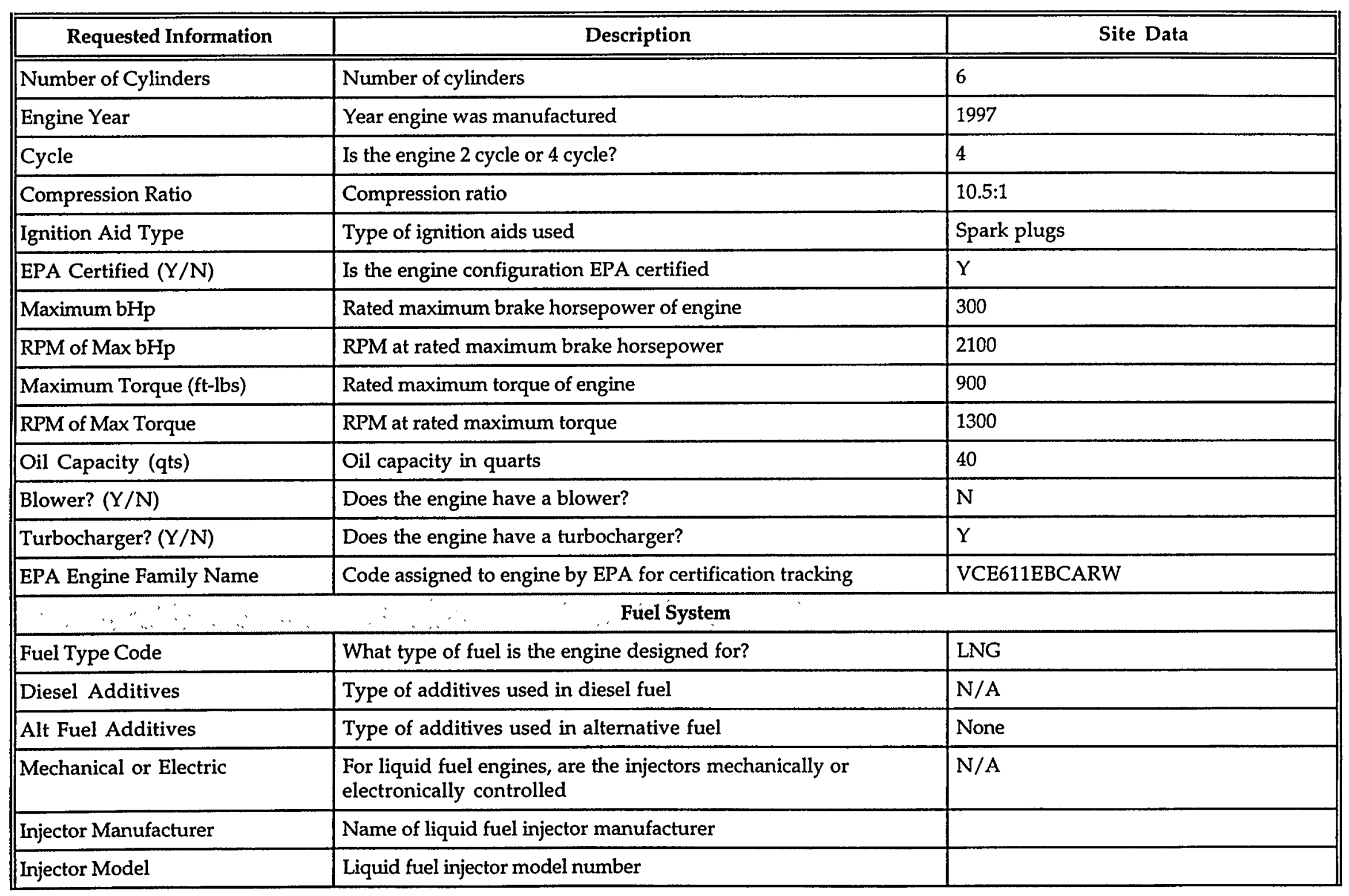


Vehicle System Descriptions (continued)

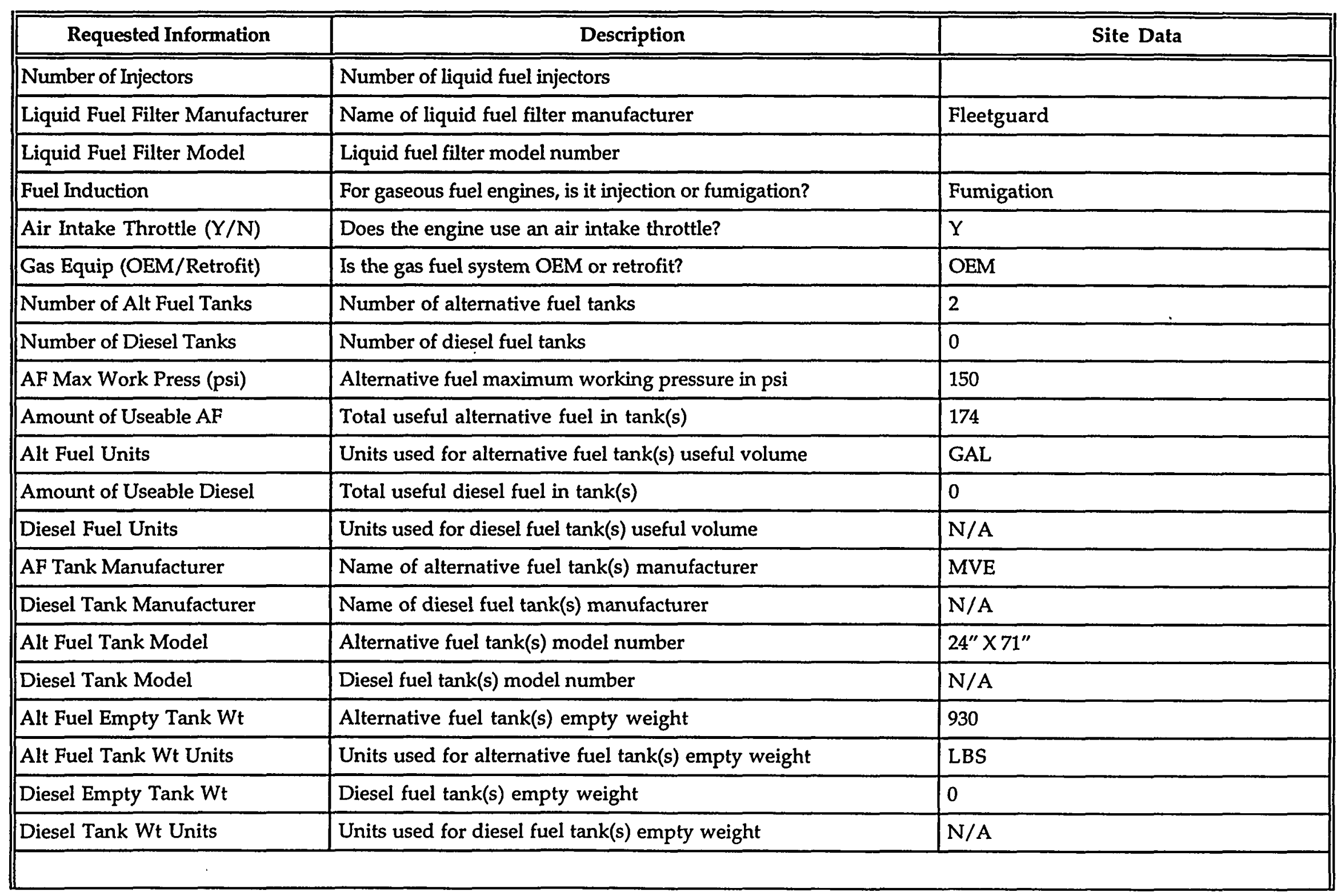


Vehicle System Descriptions (continued)

\begin{tabular}{|c|c|c|}
\hline Requested Information & Description & Site Data \\
\hline Transmission Manufacturer & Name of transmission manufacturer & Fuller \\
\hline Transmission Model Number & Transmission model number & RT11710B \\
\hline Transmission Year of Mfr & Transmission year of manufacture & 1997 \\
\hline Transmission Type Code & Type of Transmission (Code 7 from VMRSH) & 1 \\
\hline Reverse Speeds & Number of reverse speeds & 2 \\
\hline Retarder $(\mathrm{Y} / \mathrm{N})$ & Is the truck equipped with a retarder/Jake brake? & $Y$ \\
\hline$\therefore \cdots \cdots$ & $\because \because \because \because \vdots \vdots$ & \\
\hline Axle Type Code & Type of axle configuration (Code 3 from VMRSH) & D \\
\hline Axle Manufacturer Code & Name of drive axle manufacturer (from VMRSH) & Eaton \\
\hline Axle Model & Drive axle model number & DS 404 \\
\hline Rear Axle Config Code & Rear axle configuration (Code 37 from VMRSH) & \\
\hline Rear Axle Setup Code & Setup of rear axle configuration (Code 38 from VMRSH) & 2 \\
\hline Axle Ratio Low & Low axle ratio & 4.11:1 \\
\hline Axle Ratio High & High axle ratio & \\
\hline Total GVW Wt (lb) & Total gross vehicle weight in pounds & 80,000 LBS \\
\hline Total Curb Wt (lb) & Total weight with the truck in curb weight configuration & 15,620 LBS \\
\hline
\end{tabular}


Vehicle System Descriptions (continued)

\begin{tabular}{||l|l|l||}
\hline \multicolumn{1}{|c|}{ Requested Information } & \multicolumn{1}{|c|}{ Description } & \multicolumn{1}{|c|}{ Site Data } \\
\hline \hline Torque Converter Ratio & Torque converter ratio & N/A \\
\hline Wheelbase & Length of wheelbase & 170 \\
\hline Catalytic Converter & Does the vehicle have a catalytic converter? & N \\
\hline Catalytic Converter Mfr & Name of catalytic converter manufacturer & \\
\hline Catalytic Converter Model & Model number of the catalytic converter & N \\
\hline Diesel Particulate Trap & Does the vehicle have a diesel particulate trap? & \\
\hline Trap Manufacturer & Name of the particulate trap manufacturer & \\
\hline Trap Model & Model number of the particulate trap & \\
\hline Trap Regeneration Type & Type of trap regeneration process & \\
\hline Trap Configuration & Particulate trap configuration & \\
\hline Number of Trap Elements & Number of particulate trap elements & \\
\hline Trap System Weight & Weight of the particulate trap system & \\
\hline
\end{tabular}

VMRSH - Vehicle Maintenance Reporting Standards Handbook from the American Trucking Associations and published by Management Systems Council. 


\section{Appendix C}

\section{Detail Trip Summary, Average Speed Summary, and Average Monthly Mileage Summary}


Vehicle Usage Statistics

$3 / 10 / 99$

\begin{tabular}{|c|c|c|c|c|c|c|c|c|c|c|c|c|c|c|c|}
\hline \multirow[b]{2}{*}{ Vehicle } & \multirow[b]{2}{*}{ Month } & \multicolumn{6}{|l|}{ Total } & \multicolumn{4}{|c|}{ Per Trip } & \multicolumn{4}{|c|}{ Per Day } \\
\hline & & Miles & Hours & Days & Trips & Stores & BkHl & Miles & Hours & Stores & $\mathrm{BkHl}$ & Miles & Hours & Stores & BkHl \\
\hline \multirow[t]{8}{*}{1501} & May & 872.1 & 54.75 & 6 & 15 & 30 & $\overline{0}$ & 58.1 & 3.7 & 2.0 & 0.0 & $\overline{145.4}$ & 9.1 & 5.0 & 0.0 \\
\hline & Jun & 3285.4 & 212.63 & 22 & 59 & 114 & 2 & 55.7 & 3.6 & 1.9 & 0.0 & 149.3 & 9.7 & 5.2 & 0.1 \\
\hline & Jul & 1745.0 & 121.82 & 14 & 33 & 68 & 3 & 52.9 & 3.7 & 2.1 & 0.1 & 124.6 & 8.7 & 4.9 & 0.2 \\
\hline & Aug & 2178.8 & 153.95 & 17 & 42 & 88 & 7 & 51.9 & 3.7 & 2.1 & 0.2 & 128.2 & 9.1 & 5.2 & 0.4 \\
\hline & Sep & 3008.5 & 223.32 & 22 & 64 & 132 & 6 & 47.0 & 3.5 & 2.1 & 0.1 & 136.8 & 10.2 & 6.0 & 0.3 \\
\hline & Oct & 4205.7 & 311.55 & 27 & 86 & 186 & 6 & 48.9 & 3.6 & 2.2 & 0.1 & 155.8 & 11.5 & 6.9 & 0.2 \\
\hline & Nov & 2917.2 & 205.10 & 19 & 58 & 116 & 4 & 50.3 & 3.5 & 2.0 & 0.1 & 153.5 & 10.8 & 6.1 & 0.2 \\
\hline & Dec & 3741.3 & 282.25 & 23 & 78 & 170 & 3 & 48.0 & 3.6 & 2.2 & 0.0 & 162.7 & 12.3 & 7.4 & 0.1 \\
\hline \multirow[t]{13}{*}{1998} & Jan & 1688.0 & 127.85 & 15 & 37 & 77 & 5 & 45.6 & 3.5 & 2.1 & 0.1 & 112.5 & 8.5 & 5.1 & 0.3 \\
\hline & Feb & 2096.3 & 158.62 & 17 & 44 & 96 & 2 & 47.6 & 3.6 & 2.2 & 0.0 & 123.3 & 9.3 & 5.6 & 0.1 \\
\hline & Mar & 3154.9 & 220.95 & 22 & 58 & 133 & 4 & 54.4 & 3.8 & 2.3 & 0.1 & 143.4 & 10.0 & 6.0 & 0.2 \\
\hline & Apr & 2287.2 & 139.88 & 15 & 33 & 76 & 3 & 69.3 & 4.2 & 2.3 & 0.1 & 152.5 & 9.3 & 5.1 & 0.2 \\
\hline & May & 3892.7 & 236.93 & 19 & 57 & 130 & 1 & 68.3 & 4.2 & 2.3 & 0.0 & 204.9 & 12.5 & 6.8 & 0.1 \\
\hline & Jun & 3916.1 & 250.33 & 23 & 64 & 146 & 0 & 61.2 & 3.9 & 2.3 & 0.0 & 170.3 & 10.9 & 6.3 & 0.0 \\
\hline & Jul & 4693.9 & 287.50 & 24 & 69 & 161 & 7 & 68.0 & 4.2 & 2.3 & 0.1 & 195.6 & 12.0 & 6.7 & 0.3 \\
\hline & Aug & 3060.2 & 233.30 & 21 & 64 & 149 & 8 & 47.8 & 3.6 & 2.3 & 0.1 & 145.7 & 11.1 & 7.1 & 0.4 \\
\hline & Sep & 1809.9 & 138.83 & 13 & 39 & 88 & 2 & 46.4 & 3.6 & 2.3 & 0.1 & 139.2 & 10.7 & 6.8 & 0.2 \\
\hline & Oct & 3115.4 & 224.35 & 21 & 60 & 139 & 4 & 51.9 & 3.7 & 2.3 & 0.1 & 148.4 & 10.7 & 6.6 & 0.2 \\
\hline & Nov & 3355.0 & 217.32 & 20 & 55 & 130 & 4 & 61.0 & 4.0 & 2.4 & 0.1 & 167.8 & 10.9 & 6.5 & 0.2 \\
\hline & Dec & 2208.5 & 148.85 & 14 & 37 & 92 & 0 & 59.7 & 4.0 & 2.5 & 0.0 & 157.8 & 10.6 & 6.6 & 0.0 \\
\hline & Total & 57232.1 & 3950.08 & 374 & 1052 & 2321 & 71 & 54.4 & 3.8 & 2.2 & 0.1 & 153.0 & 10.6 & 6.2 & 0.2 \\
\hline \multicolumn{2}{|c|}{ ince $1 / 98$} & 35278.1 & 2384.71 & 224 & 617 & 1417 & 40 & 57.2 & 3.9 & 2.3 & 0.1 & 157.5 & 10.6 & 6.3 & 0.2 \\
\hline \multicolumn{2}{|c|}{ Before 1/98 } & 21954.0 & 1565.37 & 150 & 435 & 904 & 31.0 & 50.5 & 3.6 & 2.1 & 0.1 & 146.4 & 10.4 & 6.0 & 0.2 \\
\hline \multirow[t]{8}{*}{1502} & May & 1257.7 & 82.38 & 6 & 22 & 45 & 1 & 57.2 & 3.7 & 2.0 & 0.0 & 209.6 & 13.7 & 7.5 & 0.2 \\
\hline & Jun & 4172.8 & 265.07 & 22 & 68 & 142 & 9 & 61.4 & 3.9 & 2.1 & 0.1 & 189.7 & 12.0 & 6.5 & 0.4 \\
\hline & Jul & 2945.8 & 199.55 & 20 & 52 & 113 & 6 & 56.7 & 3.8 & 2.2 & 0.1 & 147.3 & 10.0 & 5.7 & 0.3 \\
\hline & Aug & 3747.7 & 255.40 & 22 & 67 & 145 & 10 & 55.9 & 3.8 & 2.2 & 0.1 & 170.4 & 11.6 & 6.6 & 0.5 \\
\hline & Sep & 3580.8 & 260.35 & 24 & 70 & 157 & 13 & 51.2 & 3.7 & 2.2 & 0.2 & 149.2 & 10.8 & 6.5 & 0.5 \\
\hline & Oct & 4551.2 & 294.13 & 26 & 72 & 175 & 15 & 63.2 & 4.1 & 2.4 & 0.2 & 175.0 & 11.3 & 6.7 & 0.6 \\
\hline & Nov & 3056.3 & 197.73 & 19 & 49 & 115 & 13 & 62.4 & 4.0 & 2.3 & 0.3 & 160.9 & 10.4 & 6.1 & 0.7 \\
\hline & Dec & 4134.9 & 260.38 & 21 & 64 & 155 & 10 & 64.6 & 4.1 & 2.4 & 0.2 & 196.9 & 12.4 & 7.4 & 0.5 \\
\hline \multirow[t]{13}{*}{1998} & Jan & 3562.0 & 230.28 & 23 & 62 & 130 & 6 & 57.5 & 3.7 & 2.1 & 0.1 & 154.9 & 10.0 & 5.7 & 0.3 \\
\hline & Feb & 2970.0 & 201.40 & 18 & 52 & 113 & 0 & 57.1 & 3.9 & 2.2 & 0.0 & 165.0 & 11.2 & 6.3 & 0.0 \\
\hline & Mar & 2806.7 & 200.03 & 19 & 54 & 115 & 2 & 52.0 & 3.7 & 2.1 & 0.0 & 147.7 & 10.5 & 6.1 & 0.1 \\
\hline & Apr & 4589.2 & 275.70 & 24 & 66 & 145 & 4 & 69.5 & 4.2 & 2.2 & 0.1 & 191.2 & 11.5 & 6.0 & 0.2 \\
\hline & May & 4244.6 & 244.43 & 19 & 56 & 132 & 3 & 75.8 & 4.4 & 2.4 & 0.1 & 223.4 & 12.9 & 6.9 & 0.2 \\
\hline & Jun & 4499.4 & 248.35 & 23 & 57 & 138 & 6 & 78.9 & 4.4 & 2.4 & 0.1 & 195.6 & 10.8 & 6.0 & 0.3 \\
\hline & Jul & 4483.8 & 269.75 & 23 & 64 & 150 & 12 & 70.1 & 4.2 & 2.3 & 0.2 & 194.9 & 11.7 & 6.5 & 0.5 \\
\hline & Aug & 4334.4 & 231.77 & 19 & 48 & 124 & 9 & 90.3 & 4.8 & 2.6 & 0.2 & 228.1 & 12.2 & 6.5 & 0.5 \\
\hline & Sep & 3957.3 & 226.82 & 20 & 52 & 123 & 5 & 76.1 & 4.4 & 2.4 & 0.1 & 197.9 & 11.3 & 6.2 & 0.3 \\
\hline & Oct & 3638.6 & 224.87 & 21 & 58 & 128 & 8 & 62.7 & 3.9 & 2.2 & 0.1 & 173.3 & 10.7 & 6.1 & 0.4 \\
\hline & Nov & 3366.3 & 205.32 & 18 & 50 & 118 & 7 & 67.3 & 4.1 & 2.4 & 0.1 & 187.0 & 11.4 & 6.6 & 0.4 \\
\hline & Dec & 2760.4 & 182.40 & 17 & 47 & 111 & 5 & 58.7 & 3.9 & 2.4 & 0.1 & 162.4 & 10.7 & 6.5 & 0.3 \\
\hline & |Total & 72659.9 & 4556.11 & 404 & 1130 & 2574 & 144 & 64.3 & 4.0 & 2.3 & 0.1 & 179.9 & 11.3 & 6.4 & 0.4 \\
\hline \multicolumn{2}{|c|}{ Since $1 / 98$} & 45212.7 & 2741.12 & 244 & 666 & 1527 & 67 & 67.9 & 4.1 & 2.3 & 0.1 & 185.3 & 11.2 & 6.3 & 0.3 \\
\hline \multicolumn{2}{|c|}{ Before 1/98 } & 27447.2 & 1814.99 & 160 & 464 & 1047 & 77.0 & 59.2 & 3.9 & 2.3 & 0.2 & 171.5 & 11.3 & 6.5 & 0.5 \\
\hline \multirow[t]{5}{*}{1503} & May & 1219.5 & 70.88 & 6 & 16 & 36 & 2 & 76.2 & 4.4 & 2.3 & 0.1 & 203.3 & 11.8 & 6.0 & 0.3 \\
\hline & Jun & 4313.5 & 266.60 & 21 & 66 & 142 & 11 & 65.4 & 4.0 & 2.2 & 0.2 & 205.4 & 12.7 & 6.8 & 0.5 \\
\hline & Jul & 3882.9 & 244.82 & 20 & 56 & 133 & 6 & 69.3 & 4.4 & 2.4 & 0.1 & 194.1 & 12.2 & 6.7 & 0.3 \\
\hline & Aug & 2503.2 & 149.78 & 17 & 33 & 85 & 5 & 75.9 & 4.5 & 2.6 & 0.2 & 147.2 & 8.8 & 5.0 & 0.3 \\
\hline & Sep & 3634.1 & 240.90 & 21 & 56 & 139 & 6 & 64.9 & 4.3 & 2.5 & 0.1 & 173.1 & 11.5 & 6.6 & 0.3 \\
\hline
\end{tabular}


Vehicle Usage Statistics

$3 / 10 / 99$

\begin{tabular}{|c|c|c|c|c|c|c|c|c|c|c|c|c|c|c|c|}
\hline \multirow[b]{2}{*}{ Vehicle } & \multirow[b]{2}{*}{ Month } & \multicolumn{6}{|l|}{ Total } & \multicolumn{4}{|c|}{ Per Trip } & \multicolumn{4}{|l|}{ Per Day } \\
\hline & & Miles & Hours & Days & Trips & Stores & BkHI & Miles & Hours & Stores & $\mathrm{BkHl}$ & Miles & Hours & Stores & BkHl? \\
\hline \multirow{16}{*}{1998} & Oct & 5775.5 & 370.80 & 27 & 89 & 211 & 9 & 64.9 & 4.2 & 2.4 & 0.1 & 213.9 & 13.7 & 7.8 & 0.3 \\
\hline & Nov & 3976.8 & 255.53 & 23 & 64 & 145 & 4 & 62.1 & 4.0 & 2.3 & 0.1 & 172.9 & 11.1 & 6.3 & 0.2 \\
\hline & Dec & 4311.9 & 263.58 & 21 & 61 & 148 & 6 & 70.7 & 4.3 & 2.4 & 0.1 & 205.3 & 12.6 & 7.0 & 0.3 \\
\hline & Jan & 4586.2 & 290.33 & 23 & 70 & 166 & 5 & 65.5 & 4.1 & 2.4 & 0.1 & 199.4 & 12.6 & 7.2 & 0.2 \\
\hline & Feb & 3275.5 & 205.15 & 17 & 49 & 116 & 2 & 66.8 & 4.2 & 2.4 & 0.0 & 192.7 & 12.1 & 6.8 & 0.1 \\
\hline & Mar & 4619.9 & 293.95 & 23 & 69 & 168 & 3 & 67.0 & 4.3 & 2.4 & 0.0 & 200.9 & 12.8 & 7.3 & 0.1 \\
\hline & Apr & 5256.0 & 335.87 & 24 & 80 & 187 & 3 & 65.7 & 4.2 & 2.3 & 0.0 & 219.0 & 14.0 & 7.8 & 0.1 \\
\hline & May & 4331.6 & 259.08 & 20 & 60 & 142 & 4 & 72.2 & 4.3 & 2.4 & 0.1 & 216.6 & 13.0 & 7.1 & 0.2 \\
\hline & Jun & 5324.4 & 299.10 & 21 & 67 & 154 & 10 & 79.5 & 4.5 & 2.3 & 0.1 & 253.5 & 14.2 & 7.3 & 0.5 \\
\hline & Jul & 4201.6 & 229.80 & 17 & 49 & 123 & 7 & 85.7 & 4.7 & 2.5 & 0.1 & 247.2 & 13.5 & 7.2 & 0.4 \\
\hline & Aug & 4445.9 & 260.02 & 21 & 59 & 141 & 9 & 75.4 & 4.4 & 2.4 & 0.2 & 211.7 & 12.4 & 6.7 & 0.4 \\
\hline & Sep & 2117.9 & 112.70 & 10 & 25 & 56 & 3 & 84.7 & 4.5 & 2.2 & 0.1 & 211.8 & 11.3 & 5.6 & 0.3 \\
\hline & Oct & 2233.5 & 139.92 & 11 & 35 & 77 & 3 & 63.8 & 4.0 & 2.2 & 0.1 & 203.0 & 12.7 & 7.0 & 0.3 \\
\hline & Nov & 488.7 & 31.67 & 5 & 9 & 16 & 1 & 54.3 & 3.5 & 1.8 & 0.1 & 97.7 & 6.3 & 3.2 & 0.2 \\
\hline & Dec & 1571.4 & 90.28 & 10 & 23 & 50 & 1 & 68.3 & 3.9 & 2.2 & 0.0 & 157.1 & 9.0 & 5.0 & 0.1 \\
\hline & Total & 72070.0 & 4410.76 & 358 & 1036 & 2435 & 100 & 69.6 & 4.3 & 2.4 & 0.1 & 201.3 & 12.3 & 6.8 & 0.3 \\
\hline \multirow{2}{*}{\multicolumn{2}{|c|}{$\begin{array}{l}\text { Since 1/98 } \\
\text { Before 1/98 }\end{array}$}} & 42452.6 & 2547.87 & 202 & 595 & 1396 & 51 & 71.3 & 4.3 & 2.3 & 0.1 & 210.2 & 12.6 & 6.9 & 0.3 \\
\hline & & 29617.4 & 1862.89 & 156 & 441 & 1039 & 49.0 & 67.2 & 4.2 & 2.4 & 0.1 & 189.9 & 11.9 & 6.7 & 0.3 \\
\hline \multirow[t]{21}{*}{1504} & May & 1172.1 & 67.58 & 6 & 18 & 32 & 1 & 65.1 & 3.8 & 1.8 & 0.1 & 195.4 & 11.3 & 5.3 & 0.2 \\
\hline & Jun & 5645.2 & 312.78 & 20 & 76 & 156 & 4 & 74.3 & 4.1 & 2.1 & 0.1 & 282.3 & 15.6 & 7.8 & 0.2 \\
\hline & Jul & 4194.8 & 242.95 & 19 & 61 & 122 & 7 & 68.8 & 4.0 & 2.0 & 0.1 & 220.8 & 12.8 & 6.4 & 0.4 \\
\hline & Aug & 3137.3 & 202.80 & 20 & 54 & 110 & 4 & 58.1 & 3.8 & 2.0 & 0.1 & 156.9 & 10.1 & 5.5 & 0.2 \\
\hline & Sep & 4164.8 & 260.83 & 26 & 70 & 138 & 8 & 59.5 & 3.7 & 2.0 & 0.1 & 160.2 & 10.0 & 5.3 & 0.3 \\
\hline & Oct & 3234.2 & 211.22 & 20 & 52 & 122 & 8 & 62.2 & 4.1 & 2.3 & 0.2 & 161.7 & 10.6 & 6.1 & 0.4 \\
\hline & Nov & 3770.0 & 266.60 & 22 & 69 & 156 & 12 & 54.6 & 3.9 & 2.3 & 0.2 & 171.4 & 12.1 & 7.1 & 0.5 \\
\hline & Dec & 4263.6 & 312.18 & 23 & 78 & 189 & 10 & 54.7 & 4.0 & 2.4 & 0.1 & 185.4 & 13.6 & 8.2 & 0.4 \\
\hline & Jan & 3949.6 & 253.57 & 23 & 58 & 148 & 9 & 68.1 & 4.4 & 2.6 & 0.2 & 171.7 & 11.0 & 6.4 & 0.4 \\
\hline & Feb & 3670.2 & 193.92 & 14 & 38 & 104 & 10 & 96.6 & 5.1 & 2.7 & 0.3 & 262.2 & 13.9 & 7.4 & 0.7 \\
\hline & Mar & 6498.9 & 352.00 & 22 & 66 & 185 & 21 & 98.5 & 5.3 & 2.8 & 0.3 & 295.4 & 16.0 & 8.4 & 1.0 \\
\hline & Apr & 6433.3 & 346.02 & 23 & 65 & 181 & 17 & 99.0 & 5.3 & 2.8 & 0.3 & 279.7 & 15.0 & 7.9 & 0.7 \\
\hline & May & 4345.3 & 277.75 & 20 & 65 & 158 & 10 & 66.9 & 4.3 & 2.4 & 0.2 & 217.3 & 13.9 & 7.9 & 0.5 \\
\hline & Jun & 4798.2 & 292.75 & 22 & 67 & 162 & 8 & 71.6 & 4.4 & 2.4 & 0.1 & 218.1 & 13.3 & 7.4 & 0.4 \\
\hline & Jul & 5508.1 & 325.90 & 23 & 73 & 175 & 16 & 75.5 & 4.5 & 2.4 & 0.2 & 239.5 & 14.2 & 7.6 & 0.7 \\
\hline & Aug & 5655.4 & 346.88 & 24 & 77 & 189 & 8 & 73.4 & 4.5 & 2.5 & 0.1 & 235.6 & 14.5 & 7.9 & 0.3 \\
\hline & Sep & 4854.5 & 287.03 & 19 & 64 & 160 & 10 & 75.9 & 4.5 & 2.5 & 0.2 & 255.5 & 15.1 & 8.4 & 0.5 \\
\hline & Oct & 4505.5 & 277.33 & 21 & 70 & 157 & 7 & 64.4 & 4.0 & 2.2 & 0.1 & 214.5 & 13.2 & 7.5 & 0.3 \\
\hline & Nov & 4262.0 & 251.10 & 21 & 57 & 140 & 8 & 74.8 & 4.4 & 2.5 & 0.1 & 203.0 & 12.0 & 6.7 & 0.4 \\
\hline & Dec & 4099.4 & 262.52 & 19 & 60 & 153 & 4 & 68.3 & 4.4 & 2.6 & 0.1 & 215.8 & 13.8 & 8.1 & 0.2 \\
\hline & Total & 88162.4 & 5343.71 & 407 & 1238 & 2937 & 182 & 71.2 & 4.3 & 2.4 & 0.1 & 216.6 & 13.1 & 7.2 & 0.4 \\
\hline \multirow{2}{*}{\multicolumn{2}{|c|}{$\begin{array}{l}\text { Since } 1 / 98 \\
\text { Before } 1 / 98\end{array}$}} & 58580.4 & 3466.77 & 251 & 760 & 1912 & 128 & 77.1 & 4.6 & 2.5 & 0.2 & 233.4 & 13.8 & 7.6 & 0.5 \\
\hline & & 29582.0 & 1876.94 & 156 & 478 & 1025 & 54.0 & 61.9 & 3.9 & 2.1 & 0.1 & 189.6 & 12.0 & 6.6 & 0.3 \\
\hline \multirow[t]{10}{*}{1505} & May & 956.3 & 71.82 & 6 & 19 & 43 & 3 & 50.3 & 3.8 & 2.3 & 0.2 & 159.4 & 12.0 & 7.2 & 0.5 \\
\hline & $\mathrm{Ju}$ & 3534.8 & 257.67 & 20 & 65 & 151 & 5 & 54.4 & 4.0 & 2.3 & 0.1 & 176.7 & 12.9 & 7.6 & 0.3 \\
\hline & Jul & 2647.0 & 194.72 & 17 & 52 & 116 & 4 & 50.9 & 3.7 & 2.2 & 0.1 & 155.7 & 11.5 & 6.8 & 0.2 \\
\hline & Aug & 1860.1 & 175.82 & 17 & 49 & 110 & 10 & 38.0 & 3.6 & 2.2 & 0.2 & 109.4 & 10.3 & 6.5 & 0.6 \\
\hline & Sep & 1745.6 & 155.87 & 19 & 44 & 96 & 9 & 39.7 & 3.5 & 2.2 & 0.2 & 91.9 & 8.2 & 5.1 & 0.5 \\
\hline & Oct & 3553.9 & 271.42 & 24 & 71 & 167 & 13 & 50.1 & 3.8 & 2.4 & 0.2 & 148.1 & 11.3 & 7.0 & 0.5 \\
\hline & Nov & 2909.8 & 217.25 & 20 & 57 & 133 & 8 & 51.0 & 3.8 & 2.3 & 0.1 & 145.5 & 10.9 & 6.7 & 0.4 \\
\hline & Dec & 2275.0 & 180.20 & 20 & 50 & 111 & 5 & 45.5 & 3.6 & 2.2 & 0.1 & 113.8 & 9.0 & 5.6 & 0.3 \\
\hline & Jan & 1945.4 & 139.73 & 14 & 37 & 84 & 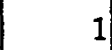 & 52.6 & 3.8 & 2.3 & 0.0 & 139.0 & 10.0 & 6.0 & 0.1 \\
\hline & Feb & 1387.1 & 88.20 & 9 & 21 & 51 & 4 & 66.1 & 4.2 & 2.4 & 0.2 & 154.1 & 9.8 & 5.7 & 0.4 \\
\hline
\end{tabular}


Vehicle Usage Statistics

$3 / 10 / 99$

\begin{tabular}{|c|c|c|c|c|c|c|c|c|c|c|c|c|c|c|c|}
\hline \multirow{2}{*}{ Vehicle } & \multirow[b]{2}{*}{ Month } & \multicolumn{6}{|l|}{ Total } & \multicolumn{4}{|c|}{ Per Trip } & \multicolumn{4}{|c|}{ Per Day } \\
\hline & & Miles & Hours & Days & Trips & Stores & BkHI & Miles & Hours & Stores & $\mathrm{BkHI}$ & Miles & Hours & Stores & BkHl \\
\hline & Mar & 3053.3 & 207.78 & 19 & 48 & 124 & 11 & $\overline{63.6}$ & $\overline{4.3}$ & 2.6 & 0.2 & $\overline{160.7}$ & 10.9 & 6.5 & $\overrightarrow{0.6}$ \\
\hline & Apr & 3775.1 & 53.38 & 21 & 59 & 152 & 10 & 64.0 & 4.3 & 2.6 & 0.2 & 179.8 & 12.1 & 7.2 & 0.5 \\
\hline & May & 3966.8 & 210.10 & 16 & 44 & 106 & 6 & 90.2 & 4.8 & 2.4 & 0.1 & 247.9 & 13.1 & 6.6 & 0.4 \\
\hline & Jun & 4984.6 & 250.53 & 23 & 52 & 118 & 4 & 95.9 & 4.8 & 2.3 & 0.1 & 216.7 & 10.9 & 5.1 & 0.2 \\
\hline & Jul & 5400.0 & 315.02 & 23 & 69 & 158 & 10 & 78.3 & 4.6 & 2.3 & 0.1 & 234.8 & 13.7 & 6.9 & 0.4 \\
\hline & Aug & 4195.6 & 258.62 & 22 & 61 & 135 & 6 & 68.8 & 4.2 & 2.2 & 0.1 & 190.7 & 11.8 & 6.1 & 0.3 \\
\hline & Sep & 3724.2 & 203.70 & 18 & 43 & 101 & 4 & 86.6 & 4.7 & 2.3 & 0.1 & 206.9 & 11.3 & 5.6 & 0.2 \\
\hline & Oct & 3186.4 & 166.95 & 17 & 37 & 82 & 5 & 86.1 & 4.5 & 2.2 & 0.1 & 187.4 & 9.8 & 4.8 & 0.3 \\
\hline & Nov & 3527.2 & 200.77 & 20 & 45 & 107 & 3 & 78.4 & 4.5 & 2.4 & 0.1 & 176.4 & 10.0 & 5.4 & 0.2 \\
\hline & Dec & 2965.5 & 163.90 & 17 & 36 & 85 & 5 & 82.4 & 4.6 & 2.4 & 0.1 & 174.4 & 9.6 & 5.0 & 0.3 \\
\hline & Total & 61593.7 & 3983.45 & 362 & 959 & 2230 & 126 & 64.2 & 4.2 & 2.3 & 0.1 & 170.1 & 11.0 & 6.2 & 0.3 \\
\hline \multicolumn{2}{|c|}{ Since 1/98 } & 42111.2 & 2458.68 & 219 & 552 & 1303 & 69 & 76.3 & 4.5 & 2.4 & 0.1 & 192.3 & 11.2 & 5.9 & 0.3 \\
\hline \multicolumn{2}{|c|}{ Before 1/98 } & 19482.5 & 1524.77 & 143 & 407 & 927 & 57.0 & 47.9 & 3.7 & 2.3 & 0.1 & 136.2 & 10.7 & 6.5 & 0.4 \\
\hline \multirow[t]{8}{*}{1506} & May & 1186.0 & 69.28 & 4 & 15 & 39 & 2 & 79.1 & 4.6 & 2.6 & 0.1 & 296.5 & 17.3 & 9.8 & 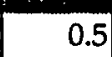 \\
\hline & Jun & 4489.2 & 307.57 & 21 & 75 & 180 & 14 & 59.9 & 4.1 & 2.4 & 0.2 & 213.8 & 14.6 & 8.6 & 0.7 \\
\hline & Jul & 3332.1 & 238.98 & 20 & 61 & 144 & 11 & 54.6 & 3.9 & 2.4 & 0.2 & 166.6 & 11.9 & 7.2 & 0. \\
\hline & Aug & 3957.0 & 286.78 & 24 & 74 & 173 & 15 & 53.5 & 3.9 & 2.3 & 0.2 & 164.9 & 11.9 & 7.2 & 0. \\
\hline & Sep & 3780.5 & 255.92 & 25 & 65 & 154 & 19 & 58.2 & 3.9 & 2.4 & 0.3 & 151.2 & 10.2 & 6.2 & 0. \\
\hline & Oct & 4780.8 & 314.03 & 26 & 81 & 185 & 17 & 59.0 & 3.9 & 2.3 & 0.2 & 183.9 & 12.1 & 7.1 & 0.2 \\
\hline & Nov & 2886.9 & 209.28 & 21 & 57 & 125 & 15 & 50.6 & 3.7 & 2.2 & 0.3 & 137.5 & 10.0 & 6.0 & 0.7 \\
\hline & Dec & 4787.7 & 269.18 & 23 & 60 & 147 & 19 & 79.8 & 4.5 & 2.5 & 0.3 & 208.2 & 11.7 & 6.4 & 0. \\
\hline \multirow[t]{13}{*}{1998} & Jan & 3788.2 & 263.93 & 24 & 63 & 165 & 5 & 60.1 & 4.2 & 2.6 & 0.1 & 157.8 & 11.0 & 6.9 & 0. \\
\hline & Feb & 4269.1 & 262.88 & 21 & 60 & 151 & 5 & 71.2 & 4.4 & 2.5 & 0.1 & 203.3 & 12.5 & 7.2 & 0. \\
\hline & Mar & 4536.2 & 289.13 & 23 & 67 & 172 & 4 & 67.7 & 4.3 & 2.6 & 0.1 & 197.2 & 12.6 & 7.5 & 0. \\
\hline & Apr & 4580.4 & 323.47 & 21 & 74 & 187 & 6 & 61.9 & 4.4 & 2.5 & 0.1 & 218.1 & 15.4 & 8.9 & U. \\
\hline & May & 3271.8 & 230.22 & 19 & 59 & 130 & 4 & 55.5 & 3.9 & 2.2 & 0.1 & 172.2 & 12.1 & 6.8 & 0.2 \\
\hline & Jun & 3363.4 & 227.32 & 20 & 57 & 128 & 4 & 59.0 & 4.0 & 2.2 & 0.1 & 168.2 & 11.4 & 6.4 & $0 .{ }^{2}$ \\
\hline & Jul & 3266.7 & 197.95 & 17 & 47 & 112 & 9 & 69.5 & 4.2 & 2.4 & 0.2 & 192.2 & 11.6 & 6.6 & 0.5 \\
\hline & Aug & 2828.6 & 182.10 & 16 & 44 & 99 & 8 & 64.3 & 4.1 & 2.3 & 0.2 & 176.8 & 11.4 & 6.2 & 0. \\
\hline & Sep & 2348.5 & 176.65 & 17 & 45 & 107 & 5 & 52.2 & 3.9 & 2.4 & 0.1 & 138.1 & 10.4 & 6.3 & 0. \\
\hline & Oct & 3165.2 & 202.45 & 18 & 51 & 113 & 5 & 62.1 & 4.0 & 2.2 & 0.1 & 175.8 & 11.2 & 6.3 & 0.3 \\
\hline & Nov & 3236.9 & 211.00 & 19 & 52 & 118 & 8 & 62.2 & 4.1 & 2.3 & 0.2 & 170.4 & 11.1 & 6.2 & 0. \\
\hline & Dec & 2313.5 & 165.15 & 16 & 42 & 96 & 4 & 55.1 & 3.9 & 2.3 & 0.1 & 144.6 & 10.3 & 6.0 & 0. \\
\hline & Total & 70168.7 & 4683.27 & 395 & 1149 & 2725 & 179 & 61.1 & 4.1 & 2.4 & 0.2 & 177.6 & 11.9 & 6.9 & 0.5 \\
\hline \multicolumn{2}{|c|}{ Since 1/98 } & 40968.5 & 2732.25 & 231 & 661 & 1578 & 67 & 62.0 & 4.1 & 2.4 & 0.1 & 177.4 & 11.8 & 6.8 & 0.3 \\
\hline \multicolumn{2}{|c|}{ Before 1/98 } & 29200.2 & 1951.02 & 164 & 488 & 1147 & 112.0 & 59.8 & 4.0 & 2.4 & 0.2 & 178.1 & 11.9 & 7.0 & 0.7 \\
\hline \multirow[t]{15}{*}{1507} & May & 1902.8 & 85.25 & 6 & 16 & 41 & 4 & 118.9 & 5.3 & 2.6 & 0.3 & 317.1 & 14.2 & 6.8 & 0.7 \\
\hline & Jun & 5307.6 & 273.42 & 19 & 52 & 146 & 14 & 102.1 & 5.3 & 2.8 & 0.3 & 279.3 & 14.4 & 7.7 & 0. \\
\hline & Jul & 4547.3 & 229.47 & 15 & 41 & 118 & 13 & 110.9 & 5.6 & 2.9 & 0.3 & 303.2 & 15.3 & 7.9 & 0. \\
\hline & Aug & 4071.7 & 213.05 & 17 & 39 & 113 & 10 & 104.4 & 5.5 & 2.9 & 0.3 & 239.5 & 12.5 & 6.6 & 0.6 \\
\hline & Sep & 2765.4 & 155.20 & 18 & 32 & 87 & 7 & 86.4 & 4.9 & 2.7 & 0.2 & 153.6 & 8.6 & 4.8 & 0. \\
\hline & Oct & 5720.6 & 297.17 & 26 & 57 & 161 & 21 & 100.4 & 5.2 & 2.8 & 0.4 & 220.0 & 11.4 & 6.2 & 0.8 \\
\hline & Nov & 5069.6 & 255.55 & 23 & 51 & 134 & 11 & 99.4 & 5.0 & 2.6 & 0.2 & 220.4 & 11.1 & 5.8 & 0. \\
\hline & Dec & 6536.7 & 327.55 & 24 & 62 & 174 & 11 & 105.4 & 5.3 & 2.8 & 0.2 & 272.4 & 13.6 & 7.3 & 0.5 \\
\hline & Jan & 4281.8 & 252.15 & 23 & 54 & 151 & 9 & 79.3 & 4.7 & 2.8 & 0.2 & 186.2 & 11.0 & 6.6 & 0.4 \\
\hline & Feb & 3107.2 & 188.37 & 17 & 45 & 106 & 4 & 69.0 & 4.2 & 2.4 & 0.1 & 182.8 & 11.1 & 6.2 & 0.2 \\
\hline & Mar & 3504.7 & 217.37 & 20 & 51 & 129 & 4 & 68.7 & 4.3 & 2.5 & 0.1 & 175.2 & 10.9 & 6.5 & 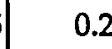 \\
\hline & Apr & 3544.0 & 235.02 & 22 & 60 & 137 & 11 & 59.1 & 3.9 & 2.3 & 0.2 & 161.1 & 10.7 & 6.2 & 1 \\
\hline & May & 3584.1 & 230.90 & 18 & 58 & 123 & 4 & 61.8 & 4.0 & 2.1 & 0.1 & 199.1 & 12.8 & 6.8 & 0.2 \\
\hline & Jun & 5155.2 & 304.28 & 22 & 67 & 168 & 8 & 76.9 & 4.5 & 2.5 & 0.1 & 234.3 & 13.8 & 7.6 & 0.4 \\
\hline & Jul & 3611.5 & 227.43 & 19 & 56 & 124 & 4 & 64.5 & 4.1 & 2.2 & 0.1 & 190.1 & 12.0 & 6.5 & 0.2 \\
\hline
\end{tabular}


\begin{tabular}{|l|l|l|l|l} 
& Potal Der Day
\end{tabular}

\begin{tabular}{|c|c|c|c|c|c|c|c|c|c|c|c|c|c|c|c|}
\hline Vehicle & Month & Miles & Hours & Days & Trips & Stores & BkHI & Miles & Hours & Stores & BkHI & Miles & Hours & Stores & BkHl \\
\hline & Aug & 6019.3 & 331.37 & 22 & 68 & 176 & 11 & 88.5 & 4.9 & 2.6 & 0.2 & 273.6 & 15.1 & 8.0 & 0.5 \\
\hline & Sep & 4513.8 & 267.30 & 17 & 62 & 145 & 4 & 72.8 & 4.3 & 2.3 & 0.1 & 265.5 & 15.7 & 8.5 & 0.2 \\
\hline & Oct & 4126.0 & 241.35 & 20 & 61 & 131 & 4 & 67.6 & 4.0 & 2.1 & 0.1 & 206.3 & 12.1 & 6.6 & 0.2 \\
\hline & Nov & 3151.8 & 202.70 & 19 & 49 & 115 & 9 & 64.3 & 4.1 & 2.3 & 0.2 & 165.9 & 10.7 & 6.1 & 0.5 \\
\hline & Dec & 3340.6 & 205.68 & 18 & 50 & 117 & 6 & 66.8 & 4.1 & 2.3 & 0.1 & 185.6 & 11.4 & 6.5 & 0.3 \\
\hline & Total & 83861.7 & 4740.58 & 385 & 1031 & 2596 & 169 & 81.3 & 4.6 & 2.5 & 0.2 & 217.8 & 12.3 & 6.7 & 0.4 \\
\hline \multirow{2}{*}{\multicolumn{2}{|c|}{$\begin{array}{l}\text { Since } 1 / 98 \\
\text { Before } 1 / 98\end{array}$}} & 47940.0 & 2903.92 & 237 & 681 & 1622 & 78 & 70.4 & 4.3 & 2.4 & 0.1 & 202.3 & 12.3 & 6.8 & 0.3 \\
\hline & & 35921.7 & 1836.66 & 148 & 350 & 974 & 91.0 & 102.6 & 5.2 & 2.8 & 0.3 & 242.7 & 12.4 & 6.6 & 0.6 \\
\hline \multirow[t]{8}{*}{1508} & May & 1484.7 & 86.57 & 6 & 22 & 43 & 1 & 67.5 & 3.9 & 2.0 & 0.0 & 247.5 & 14.4 & 7.2 & 0.2 \\
\hline & Jun & 4377.1 & 258.23 & 19 & 63 & 130 & 13 & 69.5 & 4.1 & 2.1 & 0.2 & 230.4 & 13.6 & 6.8 & 0.7 \\
\hline & Jul & 3939.9 & 255.68 & 20 & 63 & 140 & 13 & 62.5 & 4.1 & 2.2 & 0.2 & 197.0 & 12.8 & 7.0 & 0.7 \\
\hline & Aug & 4004.9 & 249.20 & 22 & 60 & 131 & 17 & 66.7 & 4.2 & 2.2 & 0.3 & 182.0 & 11.3 & 6.0 & 0.8 \\
\hline & Sep & 3580.6 & 251.00 & 23 & 66 & 140 & 8 & 54.3 & 3.8 & 2.1 & 0.1 & 155.7 & 10.9 & 6.1 & 0.3 \\
\hline & Oct & 6197.5 & 339.35 & 25 & 79 & 177 & 9 & 78.4 & 4.3 & 2.2 & 0.1 & 247.9 & 13.6 & 7.1 & 0.4 \\
\hline & Nov & 4586.6 & 275.03 & 23 & 72 & 143 & 6 & 63.7 & 3.8 & 2.0 & 0.1 & 199.4 & 12.0 & 6,2 & 0.3 \\
\hline & Dec & 5790.5 & 306.38 & 23 & 70 & 155 & 5 & 82.7 & 4.4 & 2.2 & 0.1 & 251.8 & 13.3 & 6.7 & 0.2 \\
\hline \multirow[t]{13}{*}{1998} & Jan & 4025.6 & 221.10 & 21 & 52 & 118 & 6 & $\pi 7.4$ & 4.3 & 2.3 & 0.1 & 191.7 & 10.5 & 5.6 & 0.3 \\
\hline & Feb & 3896.5 & 222.92 & 20 & 52 & 123 & 4 & 74.9 & 4.3 & 2.4 & 0.1 & 194.8 & 11.1 & 6.2 & 0.2 \\
\hline & Mar & 4009.4 & 239.20 & 22 & 57 & 129 & 6 & 70.3 & 4.2 & 2.3 & 0.1 & 182.2 & 10.9 & 5.9 & 0.3 \\
\hline & Apr & 2794.4 & 160.63 & 14 & 34 & 83 & 6 & 82.2 & 4.7 & 2.4 & 0.2 & 199.6 & 11.5 & 5.9 & 0.4 \\
\hline & May & 1271.7 & 58.50 & 4 & 11 & 28 & 0 & 115.6 & 5.3 & 2.5 & 0.0 & 317.9 & 14.6 & 7.0 & 0.0 \\
\hline & |Jun & 4315.1 & 236.28 & 19 & 54 & 116 & 8 & 79.9 & 4.4 & 2.1 & 0.1 & 227.1 & 12.4 & 6.1 & 0.4 \\
\hline & Jul & 2904.3 & 160.43 & 16 & 36 & 79 & 5 & 80.7 & 4.5 & 2.2 & 0.1 & 181.5 & 10.0 & 4.9 & 0.3 \\
\hline & Aug & 4440.8 & 240.82 & 21 & 54 & 118 & 6 & 82.2 & 4.5 & 2.2 & 0.1 & 211.5 & 11.5 & 5.6 & 0.3 \\
\hline & Sep & 3177.4 & 161.17 & 15 & 34 & 75 & 7 & 93.5 & 4.7 & 2.2 & 0.2 & 211.8 & 10.7 & 5.0 & 0.5 \\
\hline & Oct & 2860.2 & 162.53 & 15 & 41 & 83 & 1 & 69.8 & 4.0 & 2.0 & 0.0 & 190.7 & 10.8 & 5.5 & 0.1 \\
\hline & Nov & 3365.1 & 190.17 & 18 & 47 & 97 & 1 & 71.6 & 4.0 & 2.1 & 0.0 & 187.0 & 10.6 & 5.4 & 0.1 \\
\hline & Dec & 2829.0 & 158.30 & 16 & 35 & 81 & 4 & 80.8 & 4.5 & 2.3 & 0.1 & 176.8 & 9.9 & 5.1 & 0.3 \\
\hline & Total & 73851.3 & 4233.49 & 362 & 1002 & 2189 & 126 & 73.7 & 4.2 & 2.2 & 0.1 & 204.0 & 11.7 & 6.0 & 0.3 \\
\hline \multicolumn{2}{|c|}{ Since 1/98 } & 39889.5 & 2212.05 & 201 & 507 & 1130 & 54 & 78.7 & 4.4 & 2.2 & 0.1 & 198.5 & 11.0 & 5.6 & 0.3 \\
\hline \multicolumn{2}{|c|}{ Before $1 / 98$} & 33961.8 & 2021.44 & 161 & 495 & 1059 & 72.0 & 68.6 & 4.1 & 2.1 & 0.1 & 210.9 & 12.6 & 6.6 & 0.4 \\
\hline \multirow[t]{20}{*}{ LNG } & lay & 051.2 & 588.51 & 46 & 143 & 309 & 14 & 70.3 & 4.1 & 2.2 & 0.1 & 218.5 & 12.8 & 6.7 & 0.3 \\
\hline & |Ju & 35125.6 & 2153.97 & 164 & 524 & 1161 & 72 & 67.0 & 4.1 & 2.2 & 0.1 & 214.2 & 13.1 & 7.1 & 0.4 \\
\hline & $\mathrm{Ju}$ & 27234.8 & 1727.99 & 145 & 419 & 954 & 63 & 65.0 & 4.1 & 2.3 & 0.2 & 187.8 & 11.9 & 6.6 & 0.4 \\
\hline & Aug & 25460.7 & 1686.78 & 156 & 418 & 955 & 78 & 60.9 & 4.0 & 2.3 & 0.2 & 163.2 & 10.8 & 6.1 & 0.5 \\
\hline & Sep & 26260.3 & 1803.39 & 178 & 467 & 1043 & 76 & 56.2 & 3.9 & 2.2 & 0.2 & 147.5 & 10.1 & 5.9 & 0.4 \\
\hline & Oct & 38019.4 & 2409.67 & 201 & 587 & 1384 & 98 & 64.8 & 4.1 & 2.4 & 0.2 & 189.2 & 12.0 & 6.9 & 0.5 \\
\hline & Nov & 29173.2 & 1882.07 & 170 & 477 & 1067 & 73 & 61.2 & 3.9 & 2.2 & 0.2 & 171.6 & 11.1 & 6.3 & 0.4 \\
\hline & Dec & 35841.6 & 2201.70 & 178 & 523 & 1249 & 69 & 68.5 & 4.2 & 2.4 & 0.1 & 201.4 & 12.4 & 7.0 & 0.4 \\
\hline & $\operatorname{Jan}$ & 27826.8 & 1778.94 & 166 & 433 & 1039 & 46 & 64.3 & 4.1 & 2.4 & 0.1 & 167.6 & 10.7 & 6.3 & 0.3 \\
\hline & Feb & 24671.9 & 1521.46 & 133 & 361 & 860 & 31 & 68.3 & 4.2 & 2.4 & 0.1 & 185.5 & 11.4 & 6.5 & 0.2 \\
\hline & Mar & 32184.0 & 2020.41 & 170 & 470 & 1155 & 55 & 68.5 & 4.3 & 2.5 & 0.1 & 189.3 & 11.9 & 6.8 & 0.3 \\
\hline & Apr & 33259.6 & 2069.97 & 164 & 471 & 1148 & 60 & 70.6 & 4.4 & 2.4 & 0.1 & 202.8 & 12.6 & 7.0 & 0.4 \\
\hline & May & 28908.6 & 1747.91 & 135 & 410 & 949 & 32 & 70.5 & 4.3 & 2.3 & 0.1 & 214.1 & 12.9 & 7.0 & 0.2 \\
\hline & Jun & 36356.4 & 2108.94 & 173 & 485 & 1130 & 48 & 75.0 & 4.3 & 2.3 & 0.1 & 210.2 & 12.2 & 6.5 & 0.3 \\
\hline & Jul & 34069.9 & 2013.78 & 162 & 463 & 1082 & 70 & 73.6 & 4.3 & 2.3 & 0.2 & 210.3 & 12.4 & 6.7 & 0.4 \\
\hline & Aug & 34980.2 & 2084.88 & 166 & 475 & 1131 & 65 & 73.6 & 4.4 & 2.4 & 0.1 & 210.7 & 12.6 & 6.8 & 0.4 \\
\hline & Sep & 26503.5 & 1574.20 & 129 & 364 & 855 & 40 & 72.8 & 4.3 & 2.3 & 0.1 & 205.5 & 12.2 & 6.6 & 0. \\
\hline & Oct & 26830.8 & 1639.75 & 144 & 413 & 910 & 37 & 65.0 & 4.0 & 2.2 & 0.1 & 186.3 & 11.4 & 6.3 & $0.0^{\circ}$ \\
\hline & Nov & 24753.0 & 1510.05 & 140 & 364 & 841 & 41 & 68.0 & 4.1 & 2.3 & 0.1 & 176.8 & 10.8 & 6.0 & 0.3 \\
\hline & Dec & 22088.3 & 1377.08 & 127 & 330 & 785 & 29 & 66.9 & 4.2 & 2.4 & 0.1 & 173.9 & 10.8 & 6.2 & 0.2 \\
\hline
\end{tabular}


Vehicle Usage Statistics

$3 / 10 / 99$

\begin{tabular}{|l|l|l|l|l|l|l|l|l|l|l}
\hline & Total & Per Trip & Pay \\
\hline
\end{tabular}

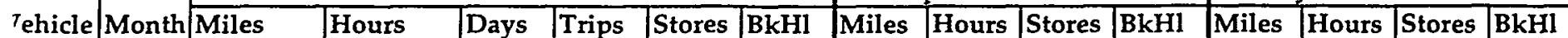

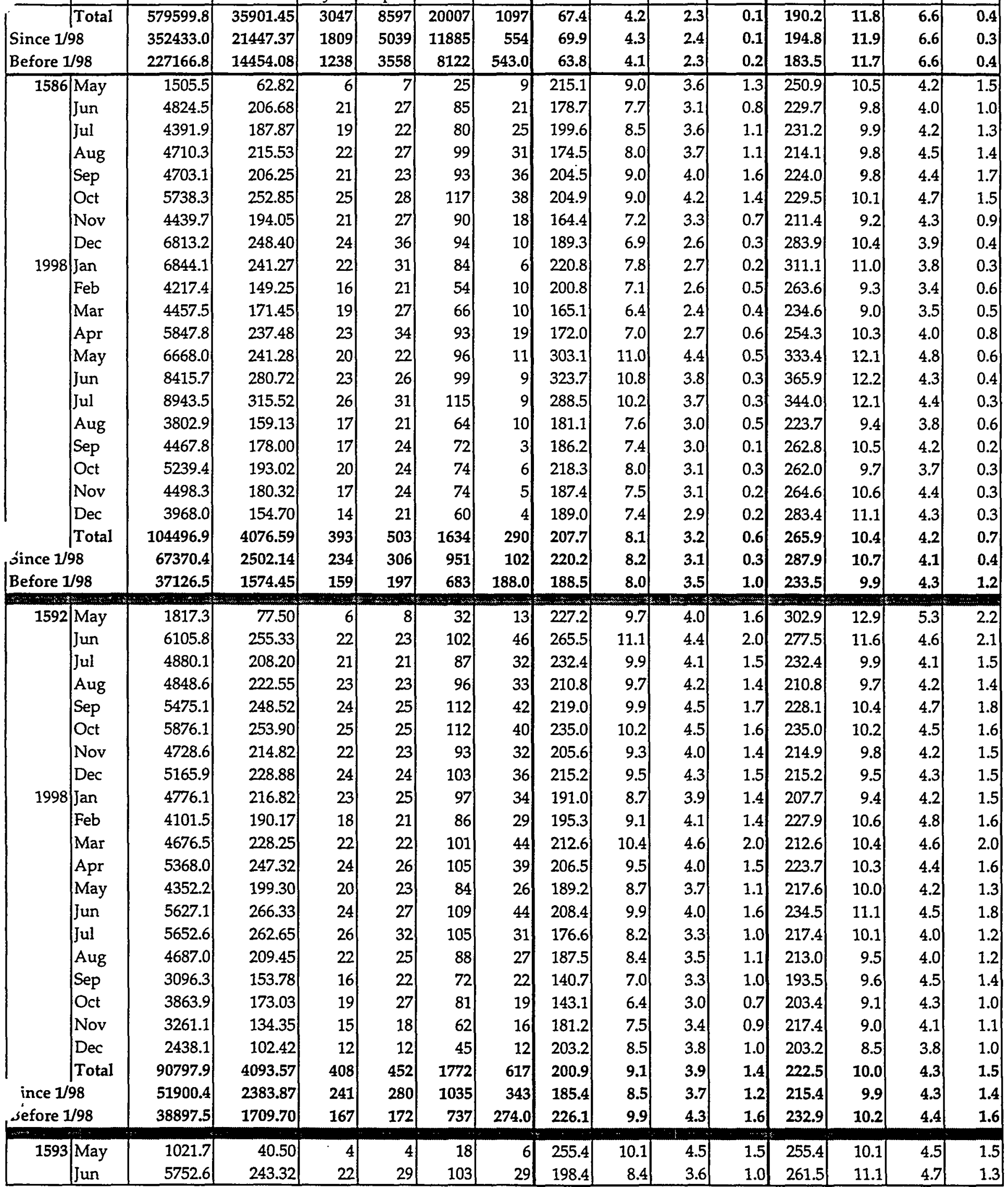


Vehicle Usage Statistics

$3 / 10 / 99$

\begin{tabular}{|c|c|c|c|c|c|c|c|c|c|c|c|c|c|c|c|}
\hline \multirow[b]{2}{*}{ Vehicle } & \multirow[b]{2}{*}{ Month } & \multicolumn{6}{|l|}{ Total } & \multicolumn{4}{|c|}{ Per Trip } & \multicolumn{4}{|l|}{ Per Day } \\
\hline & & Miles & Hours & Days & Trips & Stores & BkHI & Miles & Hours & \begin{tabular}{|l|} 
Stores \\
\end{tabular} & BkHl & Miles & Hours & Stores & $\mathrm{BkHl}$ \\
\hline & Jul & 5146.2 & 216.18 & 19 & 26 & 91 & 25 & 197.9 & 8.3 & 3.5 & 1.0 & 270.9 & 11.4 & 4.8 & $\overrightarrow{1.3}$ \\
\hline & Aug & 5868.9 & 250.13 & 24 & 27 & 109 & 37 & 217.4 & 9.3 & 4.0 & 1.4 & 244.5 & 10.4 & 4.5 & 1.5 \\
\hline & Sep & 5441.4 & 244.08 & 23 & 25 & 116 & 46 & 217.7 & 9.8 & 4.6 & 1.8 & 236.6 & 10.6 & 5.0 & 2.0 \\
\hline & Oct & 5342.3 & 232.78 & 23 & 25 & 105 & 42 & 213.7 & 9.3 & 4.2 & 1.7 & 232.3 & 10.1 & 4.6 & 1.8 \\
\hline & Nov & 5712.2 & 221.58 & 22 & 25 & 90 & 31 & 228.5 & 8.9 & 3.6 & 1.2 & 259.6 & 10.1 & 4.1 & 1.4 \\
\hline & Dec & 5263.8 & 219.67 & 21 & 25 & 99 & 28 & 210.6 & 8.8 & 4.0 & 1.1 & 250.7 & 10.5 & 4.7 & 1.3 \\
\hline 1998 & Jan & 4503.4 & 182.42 & 18 & 18 & 79 & 21 & 250.2 & 10.1 & 4.4 & 1.2 & 250.2 & 10.1 & 4.4 & 1.2 \\
\hline & Feb & 3878.5 & 161.82 & 16 & 16 & 73 & 22 & 242.4 & 10.1 & 4.6 & 1.4 & 242.4 & 10.1 & 4.6 & 1.4 \\
\hline & Mar & 4061.5 & 166.03 & 17 & 19 & 74 & 20 & 213.8 & 8.7 & 3.9 & 1.1 & 238.9 & 9.8 & 4.4 & 1.2 \\
\hline & Apr & 5159.0 & 197.18 & 19 & 23 & 79 & 16 & 224.3 & 8.6 & 3.4 & 0.7 & 271.5 & 10.4 & 4.2 & 0.8 \\
\hline & May & 5367.2 & 214.75 & 20 & 24 & 92 & 16 & 223.6 & 8.9 & 3.8 & 0.7 & 268.4 & 10.7 & 4.6 & 0.8 \\
\hline & Jun & 6638.4 & 263.20 & 24 & 30 & 108 & 17 & 221.3 & 8.8 & 3.6 & 0.6 & 276.6 & 11.0 & 4.5 & 0.7 \\
\hline & Jul & 7847.6 & 307.98 & 26 & 37 & 131 & 20 & 212.1 & 8.3 & 3.5 & 0.5 & 301.8 & 11.8 & 5.0 & 0.8 \\
\hline & Aug & 6385.0 & 256.68 & 24 & 28 & 110 & 20 & 228.0 & 9.2 & 3.9 & 0.7 & 266.0 & 10.7 & 4.6 & 0.8 \\
\hline & Sep & 4365.0 & 173.85 & 17 & 18 & 74 & 16 & 242.5 & 9.7 & 4.1 & 0.9 & 256.8 & 10.2 & 4.4 & 0.9 \\
\hline & Oct & 3787.9 & 148.45 & 14 & 14 & 64 & 18 & 270.6 & 10.6 & 4.6 & 1.3 & 270.6 & 10.6 & 4.6 & 1.3 \\
\hline & Nov & 4400.1 & 172.55 & 17 & 20 & 72 & 13 & 220.0 & 8.6 & 3.6 & 0.7 & 258.8 & 10.2 & 4.2 & 0.8 \\
\hline & Dec & 4134.5 & 163.08 & 16 & 16 & 69 & 17 & 258.4 & 10.2 & 4.3 & 1.1 & 258.4 & 10.2 & 4.3 & 1.1 \\
\hline & Total & 100077.2 & 4076.23 & 386 & 449 & 1756 & 460 & 222.9 & 9.1 & 3.9 & 1.0 & 259.3 & 10.6 & 4.5 & 1.2 \\
\hline Since 1 & & 60528.1 & 2407.99 & 228 & 263 & 1025 & 216 & 230.1 & 9.2 & 3.9 & 0.8 & 265.5 & 10.6 & 4.5 & 0.9 \\
\hline Before 1 & & 39549.1 & 1668.24 & 158 & 186 & 731 & 244.0 & 212.6 & 9.0 & 3.9 & 1.3 & 250.3 & 10.6 & 4.6 & 1.5 \\
\hline Diesel & May & 44.5 & 0.82 & 16 & 19 & 75 & 28 & 228.7 & 9.5 & 3.9 & 1.5 & 271.5 & 11.3 & 4.7 & 1.8 \\
\hline & Jun & 16682.9 & 705.33 & 65 & 79 & 290 & 96 & 211.2 & 8.9 & 3.7 & 1.2 & 256.7 & 10.9 & 4.5 & 1.5 \\
\hline & Jul & 14418.2 & 612.25 & 59 & 69 & 258 & 82 & 209.0 & 8.9 & 3.7 & 1.2 & 244.4 & 10.4 & 4.4 & $1 . \dot{q}$ \\
\hline & Aug & 15427.8 & 688.21 & 69 & 77 & 304 & 101 & 200.4 & 8.9 & 3.9 & 1.3 & 223.6 & 10.0 & 4.4 & 1.5 \\
\hline & Sep & 15619.6 & 698.85 & 68 & 73 & 321 & 124 & 214.0 & 9.6 & 4.4 & 1.7 & 229.7 & 10.3 & 4.7 & 1.8 \\
\hline & Oct & 16956.7 & 739.53 & 73 & 78 & 334 & 120 & 217.4 & 9.5 & 4.3 & 1.5 & 232.3 & 10.1 & 4.6 & 1.6 \\
\hline & Nov & 14880.5 & 630.45 & 65 & 75 & 273 & 81 & 198.4 & 8.4 & 3.6 & 1.1 & 228.9 & 9.7 & 4.2 & 1.2 \\
\hline & Dec & 17242.9 & 696.95 & 69 & 85 & 296 & 74 & 202.9 & 8.2 & 3.5 & 0.9 & 249.9 & 10.1 & 4.3 & 1.1 \\
\hline 1998 & Jan & 3.6 & 51 & 63 & 74 & 260 & 61 & 217.9 & 8.7 & 3.5 & 0.8 & 255.9 & 10.2 & 4.1 & 1.0 \\
\hline & Feb & 7.4 & 24 & 50 & 58 & 213 & 61 & 210.3 & 8.6 & 3.7 & 1.1 & 243.9 & 10.0 & 4.3 & 1.2 \\
\hline & Mar & 5.5 & & 58 & 68 & 241 & 74 & 194.1 & 8.3 & 3.5 & 1.1 & 227.5 & 9.8 & 4.2 & 1.3 \\
\hline & Apr & 374.8 & 681.98 & 66 & 83 & 277 & 74 & 197.3 & 8.2 & 3.3 & 0.9 & 248.1 & 10.3 & 4.2 & 1.1 \\
\hline & May & 16387.4 & 655.33 & 60 & 69 & 272 & 53 & 237.5 & 9.5 & 3.9 & 0.8 & 273.1 & 10.9 & 4.5 & 0.9 \\
\hline & Jun & 20681.2 & 810.25 & 71 & 83 & 316 & 70 & 249.2 & 9.8 & 3.8 & 0.8 & 291.3 & 11.4 & 4.5 & 1.0 \\
\hline & Jul & 22443.7 & 15 & 78 & 100 & 351 & 60 & 224.4 & 8.9 & 3.5 & 0.6 & 287.7 & 11.4 & 4.5 & 0.8 \\
\hline & Aug & 14874.9 & 625.26 & 63 & 74 & 262 & $5 ?$ & 201.0 & 8.4 & 3.5 & 0.8 & 236.1 & 9.9 & 4.2 & 0.9 \\
\hline & Sep & 11929.1 & 505.63 & 50 & 64 & 218 & 41 & 186.4 & 7.9 & 3.4 & 0.6 & 238.6 & 10.1 & 4.4 & 0.8 \\
\hline & Oct & 12891.2 & 514.50 & 53 & 65 & 219 & 43 & 198.3 & 7.9 & 3.4 & 0.7 & 243.2 & 9.7 & 4.1 & 0.8 \\
\hline & Nov & 12159.5 & 487.22 & 49 & 62 & 208 & 34 & 196.1 & 7.9 & 3.4 & 0.5 & 248.2 & 9.9 & 4.2 & 0.7 \\
\hline & Dec & 10540.6 & 420.20 & 42 & 49 & 174 & 33 & 215.1 & 8.6 & 3.6 & 0.7 & 251.0 & 10.0 & 4.1 & 0.8 \\
\hline & Total & 295372.0 & 12246.39 & 1187 & 1404 & 5162 & 1367 & 210.4 & 8.7 & 3.7 & 1.0 & 248.8 & 10.3 & 4.3 & 1.2 \\
\hline . & & 179798.9 & 7294.00 & 703 & 849 & 3011 & 661 & 211.8 & 8.6 & 3.5 & 0.8 & 255.8 & 10.4 & 4.3 & 0.9 \\
\hline Befor & $1 / 98$ & 115573.1 & 4952.39 & 484 & 555 & 2151 & 706.0 & 208.2 & 8.9 & 3.9 & 1.3 & 238.8 & 10.2 & 4.4 & 1.5 \\
\hline
\end{tabular}

Note: Number of stores includes backhauls. 
Route/Duty Cycle

Fleet Comparison

Raley's Diesel Control and LNG Trucks

Period: May, June, July, 1997

\begin{tabular}{|l|c|c|c|c|c|c|c|}
\begin{tabular}{|r|c|c|c|} 
Diesel \\
Control
\end{tabular} & $\begin{array}{c}\text { Total } \\
\text { Mileage }\end{array}$ & $\begin{array}{c}\text { Total } \\
\text { Hours }\end{array}$ & Avg. MPH & $\begin{array}{c}\text { Mileage } \\
\text { per Day }\end{array}$ & $\begin{array}{c}\text { Trips } \\
\text { per Day }\end{array}$ & $\begin{array}{c}\text { Avg No. of } \\
\text { Stores per Trip }\end{array}$ & $\begin{array}{c}\text { Avg No. of } \\
\text { Backhaul Stops }\end{array}$ \\
\hline 1586 & 7679.9 & 171.97 & 45 & 259 & 1.2 & 2.3 & 0.9 \\
1592 & 7825.7 & 167.83 & 47 & 270 & 1.0 & 2.6 & 1.5 \\
1593 & 8250.2 & 186.83 & 44 & 268 & 1.2 & 2.7 & 1.1 \\
\hline
\end{tabular}

\begin{tabular}{|c|c|c|c|c|c|c|c|}
\hline LNG & $\begin{array}{c}\text { Total } \\
\text { Mileage }\end{array}$ & $\begin{array}{c}\text { Total } \\
\text { Hours }\end{array}$ & Avg. MPH & $\begin{array}{c}\text { Mileage } \\
\text { per Day }\end{array}$ & $\begin{array}{c}\text { Trips } \\
\text { per Day }\end{array}$ & $\begin{array}{c}\text { Avg No. of } \\
\text { Stores per Trip }\end{array}$ & $\begin{array}{c}\text { Avg No. of } \\
\text { Backhaul Stops }\end{array}$ \\
\hline 1501 & 5698.7 & 148.76 & 38 & 184 & 2.9 & 2.2 & 0.0 \\
1502 & 5477.2 & 140.82 & 39 & 183 & 3.1 & 2.1 & 0.1 \\
1503 & 6529.4 & 164.92 & 40 & 204 & 2.8 & 2.2 & 0.1 \\
1504 & 7275.7 & 182.17 & 40 & 243 & 3.5 & 2.0 & 0.1 \\
1505 & 4673.4 & 125.70 & 37 & 156 & 2.6 & 2.2 & 0.1 \\
1506 & 6180.0 & 164.22 & 38 & 206 & 3.0 & 2.3 & 0.2 \\
1507 & 5916.0 & 142.90 & 41 & 219 & 2.2 & 2.5 & 0.3 \\
1508 & 5480.7 & 134.92 & 41 & 189 & 3.0 & 2.0 & 0.1 \\
\hline
\end{tabular}


Average Speed

$4 / 20 / 99$

Fleet Comparison

Raley's Diesel Control and LNG Trucks

Period: $11 / 13 / 98$ through $11 / 21 / 98$

\begin{tabular}{|r|r|r|r|r|r|r|r|}
\hline \multicolumn{1}{|l|}{ Truck } & Total Mileage & Drive Time & Idle Time & MPH & Stores & Trips & Stores/Trip \\
\hline 1586 & 1552.0 & 2251 & 133 & 39.1 & 29 & 8 & 3.6 \\
\hline 1592 & 1320.5 & 1848 & 1 & 42.9 & 24 & 8 & 3.0 \\
\hline 1593 & 1416.1 & 2216 & 57 & 37.4 & 19 & 6 & 3.2 \\
\hline Sum/Avg. & 4288.6 & 6315 & 191 & 39.6 & 72 & 22 & 3.3 \\
\hline 1501 & 1116.1 & 1761 & 67 & 36.6 & 45 & 20 & 2.3 \\
\hline 1502 & 839.6 & 1386 & 97 & 34.0 & 33 & 15 & 2.2 \\
\hline 1504 & 1459.1 & 2261 & 17 & 38.4 & 39 & 18 & 2.2 \\
\hline 1505 & 1106.7 & 1798 & 73 & 35.5 & 36 & 15 & 2.4 \\
\hline 1506 & 1012.8 & 1606 & 4 & 37.7 & 30 & 14 & 2.1 \\
\hline 1508 & 1326.0 & 2115 & 8 & 37.5 & 29 & 15 & 1.9 \\
\hline Sum/Avg. & 6860.3 & 10927 & 266 & 36.8 & 212 & 97 & 2.2 \\
\hline
\end{tabular}


Raley's

Monthly Mileage Calculations

\begin{tabular}{|c|c|c|c|c|c|c|c|c|c|c|}
\hline \multirow[b]{2}{*}{ Vehicle } & \multirow[b]{2}{*}{$\begin{array}{l}\text { End } \\
\text { Odometer }\end{array}$} & \multirow[b]{2}{*}{ End Date } & \multicolumn{4}{|c|}{ Before 1/98 } & \multicolumn{4}{|c|}{ Since 1/98 } \\
\hline & & & $\begin{array}{l}\text { Start } \\
\text { Odometer }\end{array}$ & $\begin{array}{l}\text { Start } \\
\text { Date }\end{array}$ & $\begin{array}{l}\text { Total } \\
\text { Mileage }\end{array}$ & $\begin{array}{l}\text { Avg per } \\
\text { Month }\end{array}$ & $\begin{array}{l}\text { Start } \\
\text { Odometer }\end{array}$ & $\begin{array}{l}\text { Start } \\
\text { Date }\end{array}$ & $\begin{array}{l}\text { Total } \\
\text { Mileage }\end{array}$ & $\begin{array}{l}\text { Avg per } \\
\text { Month }\end{array}$ \\
\hline 1586 & 273792 & $12 / 31 / 98$ & 153189 & $6 / 30 / 97$ & 37115 & 6186 & 190304 & $12 / 31 / 97$ & 83488 & 6957 \\
\hline 1592 & 225511 & $12 / 31 / 98$ & 125202 & $6 / 30 / 97$ & 35420 & 5903 & 160622 & $12 / 31 / 97$ & 64889 & 5407 \\
\hline 1593 & 269861 & $12 / 31 / 98$ & 159251 & $6 / 30 / 97$ & 36424 & 6071 & 195675 & $12 / 31 / 97$ & 74186 & 6182 \\
\hline Total & & $12 / 31 / 98$ & & $6 / 30 / 97$ & 108959 & 6053 & & $12 / 31 / 97$ & 222563 & 6182 \\
\hline 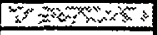 & sond & 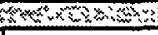 & 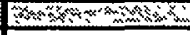 & 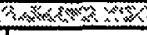 & 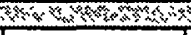 & 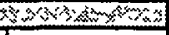 & 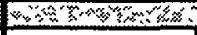 & 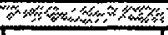 & 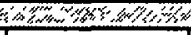 & 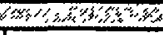 \\
\hline 1501 & 79363 & $12 / 31 / 98$ & 1365 & $4 / 30 / 97$ & 31948 & 3994 & 33313 & $12 / 31 / 97$ & 46050 & 3838 \\
\hline 1502 & 88168 & $12 / 31 / 98$ & 1413 & $4 / 30 / 97$ & 30922 & 3865 & 32335 & $12 / 31 / 97$ & 55833 & 4653 \\
\hline 1503 & 88214 & $12 / 31 / 98$ & 1318 & $4 / 30 / 97$ & 36054 & 4507 & 37372 & $12 / 31 / 97$ & 50842 & 4237 \\
\hline 1504 & 109614 & $12 / 31 / 98$ & 1318 & $4 / 30 / 97$ & 40716 & 5090 & 42034 & $12 / 31 / 97$ & 67580 & 5632 \\
\hline 1505 & 77877 & $12 / 31 / 98$ & 1135 & $4 / 30 / 97$ & 24408 & 3051 & 25543 & $12 / 31 / 97$ & 52334 & 4361 \\
\hline 1506 & 88731 & $12 / 31 / 98$ & 1423 & $4 / 30 / 97$ & 34848 & 4356 & 36271 & $12 / 31 / 97$ & 52460 & 4372 \\
\hline 1507 & 95206 & $12 / 31 / 98$ & 1446 & $4 / 30 / 97$ & 37905 & 4738 & 39351 & $12 / 31 / 97$ & 55855 & 4655 \\
\hline 1508 & 93133 & $12 / 31 / 98$ & 2165 & $4 / 30 / 97$ & 40954 & 5119 & 43119 & $12 / 31 / 97$ & 50014 & 4168 \\
\hline Total & & $12 / 31 / 98$ & & $4 / 30 / 97$ & 277755 & 4340 & & $12 / 31 / 97$ & 430968 & 4489 \\
\hline
\end{tabular}



Appendix D

Summary Statistics 


\begin{tabular}{|c|c|c|c|c|}
\hline Fleet Operations and Economics & PMA 2-3 & PMA 2-3 & PMA 4-7 & PMA 4-7 \\
\hline & \begin{tabular}{|l|} 
Diesel \\
Control \\
\end{tabular} & LNG & $\begin{array}{l}\text { Diesel } \\
\text { Control }\end{array}$ & LNG \\
\hline Number of Vehicles & 3 & 8 & 3 & 8 \\
\hline Period Used for Fuel and Oil Op Anaysis & $6 / 97-12 / 97$ & $4 / 97-12 / 97$ & $1 / 98-12 / 98$ & $1 / 98-12 / 98$ \\
\hline Total Number of Months in Period & 6.5 & 8.5 & 12 & 12 \\
\hline Fuel and Oil Analysis Base Fleet Mileage & 116,536 & 278,536 & 200,961 & 396,118 \\
\hline Period Used for Maintenance Op Analysis & $12 / 95-6 / 96$ & $8 / 97-12 / 97$ & $6 / 96-5 / 97$ & $1 / 98-12 / 98$ \\
\hline Total Number of Months in Period & 6 & 6 & 12 & 12 \\
\hline Maintenance Analysis Base Fleet Mileage & 110,201 & 198,636 & 222,844 & 438,191 \\
\hline Average Monthly Mileage per Vehicle & 6,053 & 4,340 & 6,182 & 4,489 \\
\hline Fleet Fuel Usage in Diesel \#2 Equiv. Gal. & 16,599 & 63,281 & 28,630 & 91,667 \\
\hline 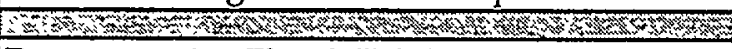 & 25is & क्षा & F & and \\
\hline Representative Fleet MPG (energy equiv) & 7.02 & 4.40 & 7.02 & 4.32 \\
\hline Ratio of MPG (AF/DC) & $28+248$ & 0.63 & 18 & 0.62 \\
\hline 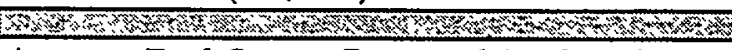 & 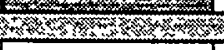 & 3y & & (1) \\
\hline Average Fuel Cost as Reported (with tax) & 1.17 & 0.74 & 1.01 & 0.65 \\
\hline & per Gal D2 & per Gal LNG & per Gal D2 & per Gal LNG \\
\hline Average Fuel Cost per Energy Equivalent & 1.17 & 1.29 & 1.01 & 1.09 \\
\hline Fuel Cost per Mile & 0.167 & 0.293 & 0.144 & 0.252 \\
\hline Q & 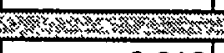 & & sis & 1 \\
\hline Total Scheduled Repair Cost per Mile & 0.018 & 0.028 & 0.021 & 0.038 \\
\hline Total Unscheduled Repair cost per Mile & 0.016 & 0.047 & 0.026 & 0.058 \\
\hline Total Maintenance Cost per Mile & 0.034 & 0.075 & 0.048 & 0.096 \\
\hline 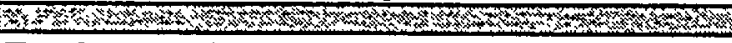 & 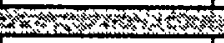 & 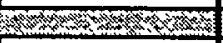 & 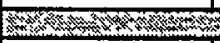 & 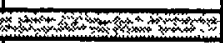 \\
\hline Total Operating Cost per Mile & 0.201 & 0.368 & 0.192 & 0.348 \\
\hline \multicolumn{5}{|l|}{ Maintenance Costs } \\
\hline & $\begin{array}{l}\text { Diesel } \\
\text { Control }\end{array}$ & LNG & \begin{tabular}{|l|} 
Diesel \\
Control
\end{tabular} & LNG \\
\hline Fleet Mileage & 110,201 & 198,636 & 222,844 & 438,191 \\
\hline 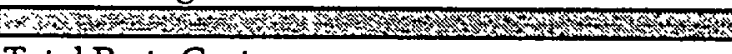 & $\sqrt{86}$ & 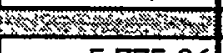 & 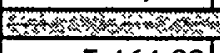 & 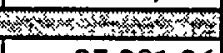 \\
\hline Total Parts Cost & 983.49 & $5,775.86$ & $5,464.09$ & $25,381.36$ \\
\hline Total Labor Hours & 55.5 & 183.2 & 103.5 & 331.7 \\
\hline Average Labor Cost & $2,775.00$ & $9,160.00$ & $5,175.00$ & $16,585.00$ \\
\hline \multicolumn{5}{|l|}{ (@ $\$ 50.00$ per hour) } \\
\hline Total Maintenance Cost & $3,758.49$ & $14,935.86$ & $10,639.09$ & $41,966.36$ \\
\hline Total Maintenance Cost per Truck & $1,252.83$ & $1,866.98$ & $3,546.36$ & $5,245.80$ \\
\hline Total Maintenance Cost per Mile & 0.034 & 0.075 & 0.048 & 0.096 \\
\hline
\end{tabular}


Breakdown of Maintenance Costs by Vehicle System

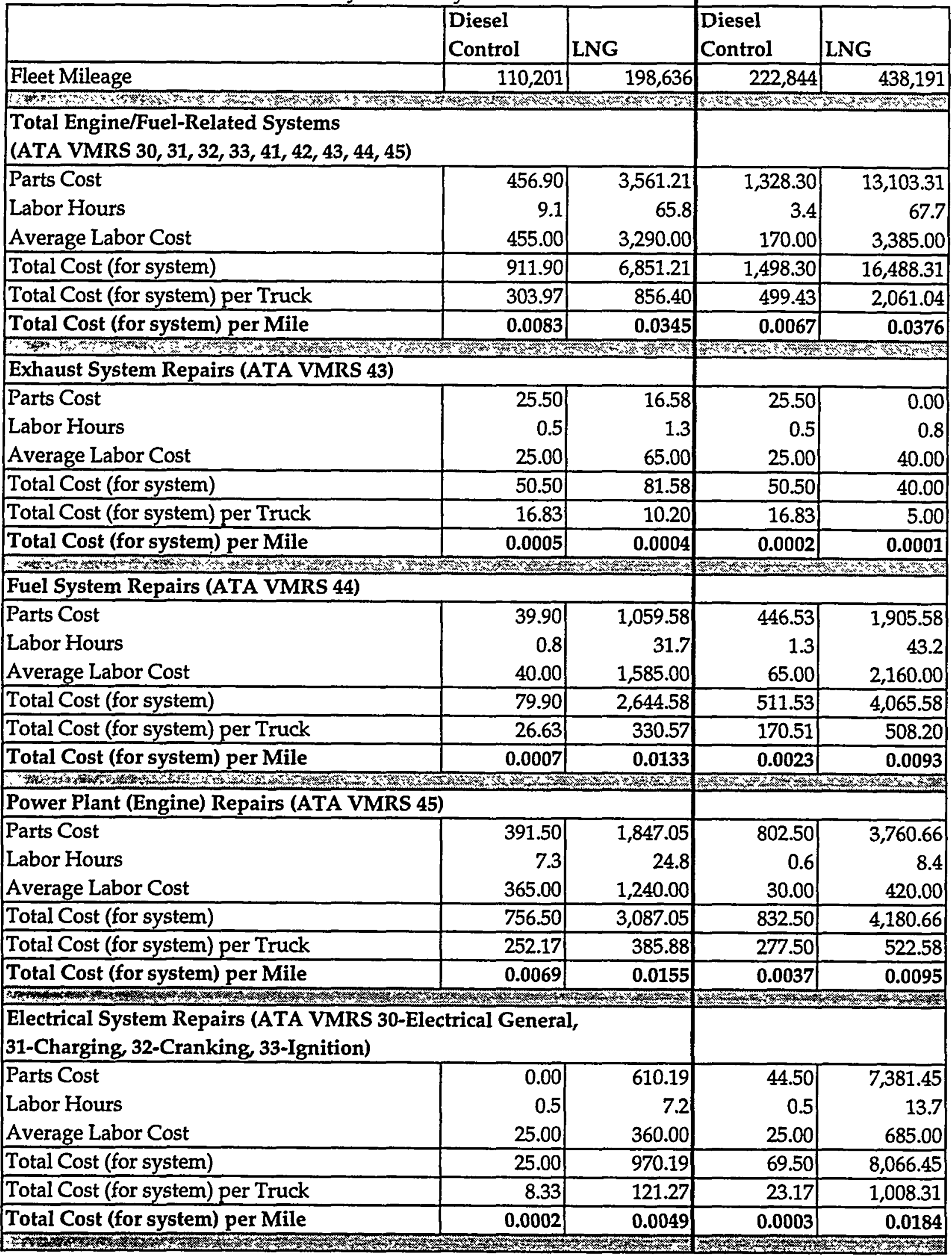


Breakdown of Maintenance Costs by Vehicle System (continued)

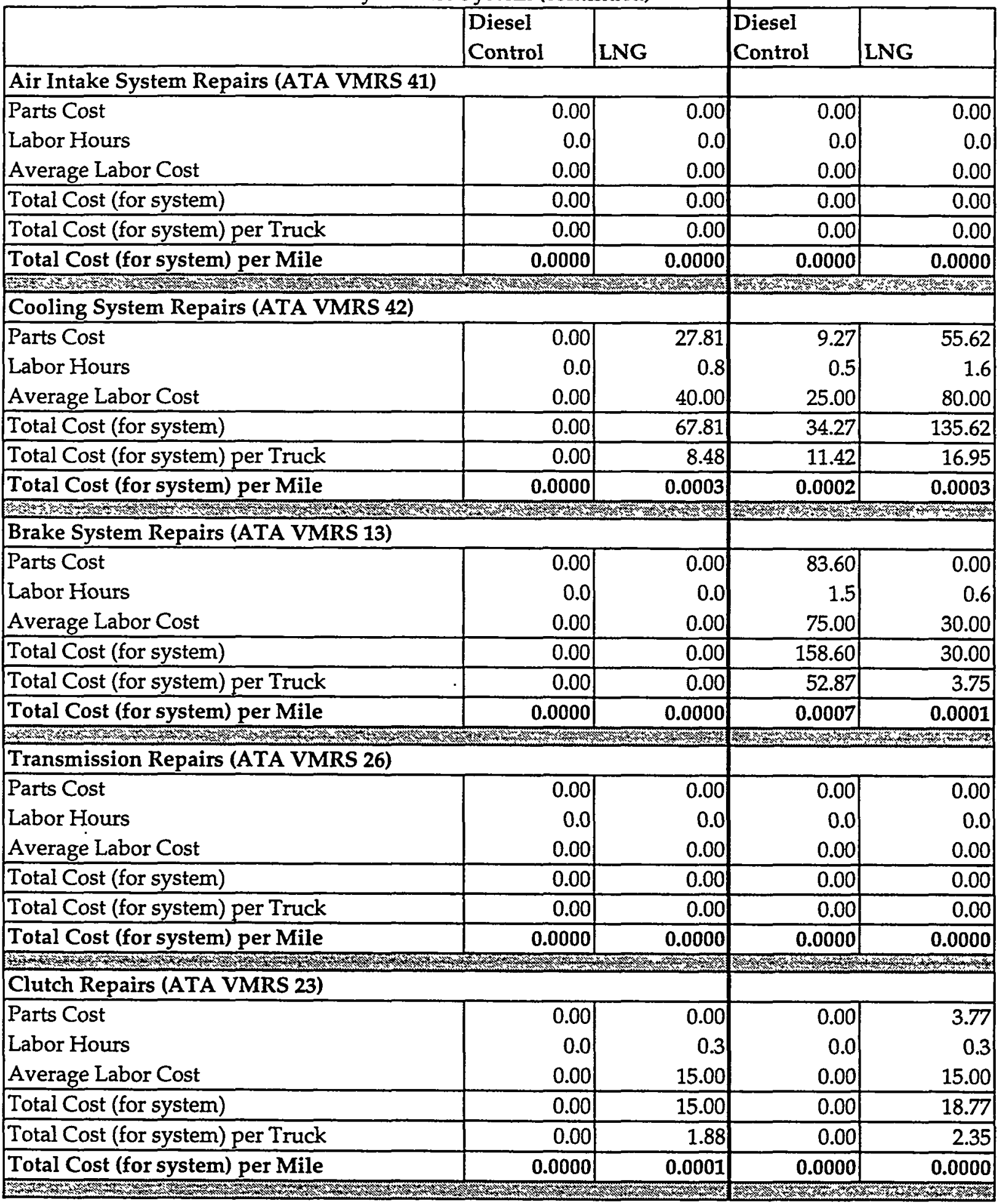


Breakdown of Maintenance Costs by Vehicle System (continued)

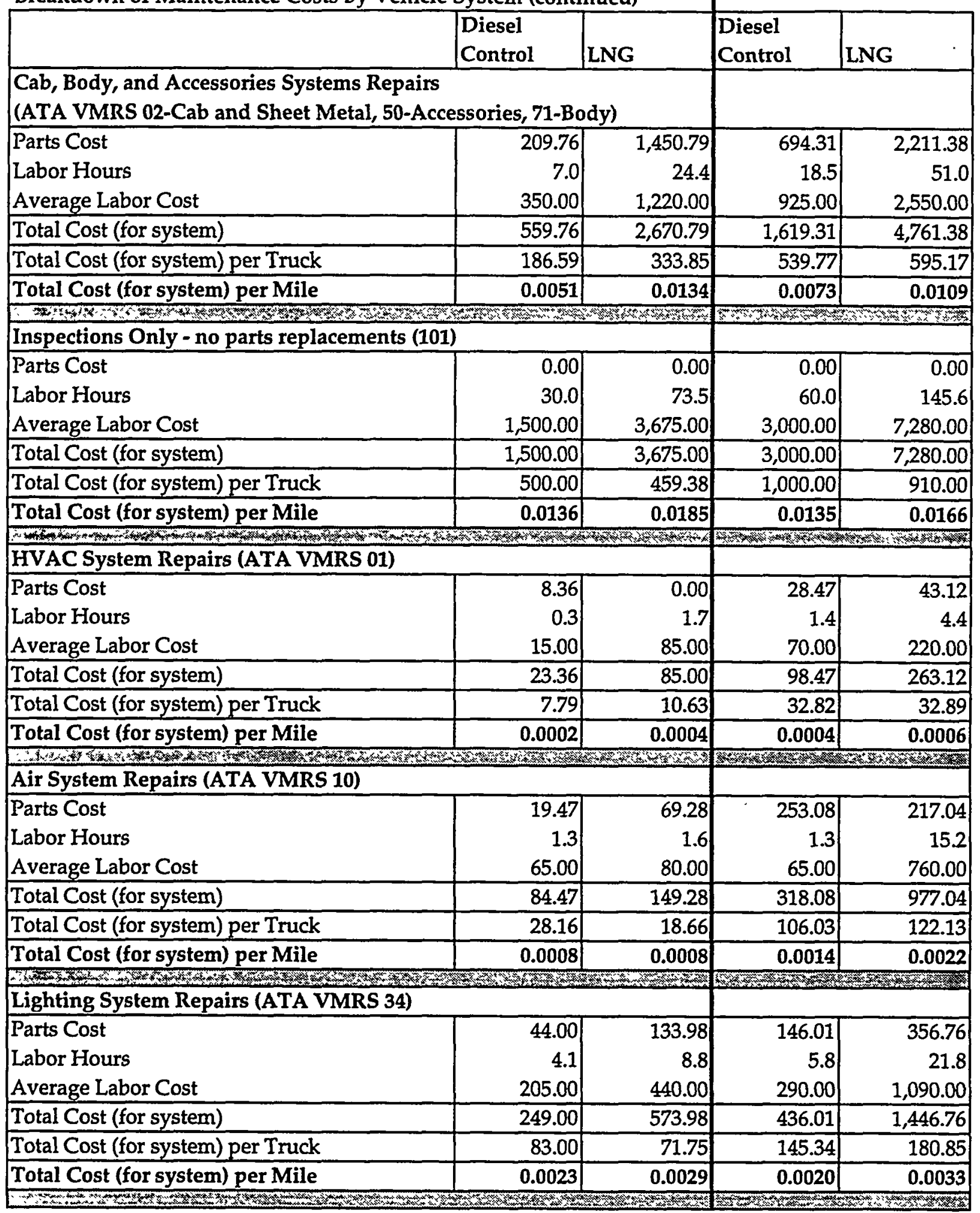


Breakdown of Maintenance Costs by Vehicle System (continued)

\begin{tabular}{|c|c|c|c|c|}
\hline & $\begin{array}{l}\text { Diesel } \\
\text { Control }\end{array}$ & LNG & $\begin{array}{l}\text { Diesel } \\
\text { Control }\end{array}$ & LNG \\
\hline \multicolumn{5}{|c|}{ Frame, Steering, and Suspension Repairs (ATA VMRS 14-Frame, 15-Steering, 16-Suspension) } \\
\hline Parts Cost & 30.00 & 80.00 & 105.32 & 317.34 \\
\hline Labor Hours & 1.3 & 0.0 & 2.3 & 3.7 \\
\hline Average Labor Cost & 65.00 & 0.00 & 115.00 & 185.00 \\
\hline Total Cost (for system) & 95.00 & 80.00 & 220.32 & 502.34 \\
\hline Total Cost (for system) per Truck & 31.67 & 10.00 & 73.44 & 62.79 \\
\hline Total Cost (for system) per Mile & 0.0009 & 0.0004 & 0.0010 & 0.0011 \\
\hline
\end{tabular}

Axle, Wheel, and Drive Shaft Repairs (ATA VMRS 11-Front Axle, 18-Wheels, 22-Rear Axle, 24-Drive Shaft)

Parts Cost

Labor Hours

Average Labor Cost

Total Cost (for system)

Total Cost (for system) per Truck

Total Cost (for system) per Mile

0.00
0.0
0.00

0.60

0

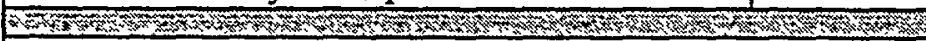

Tire Repairs (ATA VMRS 17)

Parts Cost

Labor Hours

Average Labor Cost

Total Cost (for system)

\begin{tabular}{l}
0.00 \\
\hline
\end{tabular}$\quad 40.60$

5.08

\begin{tabular}{r|r|}
0.00 & 5.08 \\
\hline
\end{tabular}

0.0002

190.00

0.0

20.14

\begin{tabular}{ll}
0.0009 & 0.0000 \\
\hline
\end{tabular}

Total Cost (for system) per Truck

Total Cost (for system) per Mile

\begin{tabular}{|r|r|r|r|}
\hline 215.00 & 480.00 & $2,635.00$ & $9,108.50$ \\
2.4 & 6.3 & 9.3 & 21.4 \\
120.00 & 315.00 & 465.00 & $1,070.00$ \\
\hline 335.00 & 795.00 & $3,100.00$ & $10,178.50$ \\
\hline 111.67 & 99.38 & $1,033.33$ & $1,272.31$ \\
\hline 0.0030 & $\mathbf{0 . 0 0 4 0}$ & $\mathbf{0 . 0 1 3 9}$ & $\mathbf{0 . 0 2 3 2}$ \\
\hline
\end{tabular}

Notes

1. The alternative fuel affected systems were chosen to include only those systems of the vehicles that could be directly impacted by the selection of an alternative fuel.

2. ATA VMRS coding is based on parts that were replaced. If there was no part replaced in a given repair, then the code was chosen by the system being worked on.

3. In general, inspections (with no part replacements) were only included in the overall totals (not by system). 101 was created to track labor costs for PMA inspections.

4. ATA VMRS 02-Cab and Sheet Metal represents seats, doors, etc.; ATA VMRS 50-Accessories represents things like fire extinguishers, test kits, etc.; ATA VMRS 71-Body represent mostly windows and windshields.

5. Average labor cost is assumed to be $\$ 50$ per hour.

6. Warranty costs are not included.

7. Diesel and LNG fuel prices shown include federal and state tax. Diesel price is calculated as a weighted average of fuel used in the analysis period. 


\section{Appendix E}

\section{Monthly Fuel and Engine Oil Consumption by Truck and Fuel Economy Analysis}


Raley's Fueling

Group Total Diesel Control

\begin{tabular}{|c|c|c|c|c|c|c|c|c|c|c|c|c|c|c|c|c|c|c|c|c|c|c|c|}
\hline & & & & & & & & & & & & & & & & & & & & & & \multirow{2}{*}{$\begin{array}{c}\text { Before } 1 / 98 \\
\text { Total }\end{array}$} & er $1 / 98$ \\
\hline & Apr-97 & May-97 & in-97 & Jul-97 & Aug-97 & Sep-97 & Oct-97 & Nov-97 & Dec-97 & \begin{tabular}{|l|l|} 
Jan-98 \\
\end{tabular} & \begin{tabular}{|l|l|} 
Feb-98 \\
\end{tabular} & Mar-98 & Apr-98 & May-98 & Jun-98 & Jul-98 & Aug-98 & Sep-98 & Oct-98 & Nov-98 & Dec-98 & & Total \\
\hline Total Mileage & & & 13,116 & 19,673 & 17,637 & 17,494 & 18,659 & 16,810 & 18,686 & 17,256 & \begin{tabular}{l|l|}
15,451 \\
\end{tabular} & 16,069 & 20,007 & 22,662 & 23,336 & 23,679 & 18,851 & 17,376 & 16,750 & 15,582 & 15,544 & 122,075 & 222,563 \\
\hline uel Mileage Base & & & 13,116 & 18,532 & 17,637 & 14,128 & 18,096 & 16,341 & 18,686 & 15,833 & 3,673 & 13,724 & 19,455 & 20,813 & 19,828 & 21,136 & 17,980 & 16,679 & 14,445 & 14,045 & 13,350 & 116,536 & 200,961 \\
\hline el (D2) & & & 1856.7 & 2622.6 & 2559.6 & 1977.4 & 2570.6 & 2340.2 & 2672.2 & 2376.2 & 1988.3 & 1979.5 & 2723.1 & 2894.2 & 2751.2 & 3015.1 & 2530.0 & 2328.3 & 2042.7 & 2095.3 & 1906.4 & 16599.2 & 28630.3 \\
\hline PG & & & 7.06 & 7.07 & 6.89 & 7.14 & 7.04 & 6.98 & 6.99 & 6.66 & 6.88 & 6.93 & 7.14 & 7.19 & 7.21 & 7.01 & 7.11 & 7.16 & 7.07 & 6.70 & 7.00 & 7.02 & 7.02 \\
\hline Const & & & 0 & 7 & 3 & 0 & 6 & 0 & 0 & 0 & 0 & 이 & 0 & & & 0 & & & & & 0 & & \\
\hline & & & & & & & & & & & & & & & & & & & & & & & \\
\hline Odometer & & & 125,202 & 131,723 & 918 & 6,861 & 12,650 & 2,766 & 8,616 & 14,281 & 19,407 & 24,740 & 30,825 & 36,650 & 43,171 & 49,564 & 55,268 & 59,950 & 65,171 & 69,418 & 73,505 & & \\
\hline lax Odometer & & & 159,251 & 165,776 & 137,065 & 143,101 & 149,573 & 19,131 & 26,304 & 33,195 & 38,750 & 44,022 & 51,206 & 60,634 & 69,660 & 79,325 & 85,437 & 91,968 & 98,728 & 104,347 & 109,792 & & \\
\hline
\end{tabular}

Group Total

LNG Trucks

Before 1/98 Since 1/98

\begin{tabular}{|c|c|c|c|c|c|c|c|c|c|c|c|c|c|c|c|c|c|c|c|c|c|c|c|}
\hline & & Maves & & & & & & & & & & & & & & & 0.9 & $20-98$ & cl-98 & |0y-98| & ec-98: & & \\
\hline otal Mileage & 00 & 38,407 & 37,751 & 32,610 & 267 & 32,173 & 102 & 31,720 & 33,723 & 34,695 & 27,109 & 40,192 & 40,276 & 39,507 & 41,215 & 37,058 & 38,180 & 35,960 & 35,657 & 29,900 & 31,221 & 288,239 & 30,970 \\
\hline 3ar & 486 & ,086 & 36,035 & 254 & 730 & 30,5 &, 150 & 36 & 32,7 & 3,665 & 24, & 7,274 & 36,194 & 5,345 & 8,342 & 31,879 & 37,089 & 34,282 & 31,892 & 25,909 & 1 & 336] & 396,118 \\
\hline & & 49,480 & 47,897 & 42,640 & 40,760 & 40,913 & 50,076 & 41,254 & 44,589 & 45,106 & 3,062 & 49,712 & 48,042 & 46,952 & 50,469 & 43,889 & 53,905 & 47,148 & 39,571 & 666 & 35, & 145| & 528,757 \\
\hline & 72.8 & $14,017.0$ & $13,568.6$ & $12,079.2$ & $11,546.7$ & $11,590.1$ & $14,185.9$ & $11,686.8$ & $12,631.5$ & $12,778.0$ & $9,365.9$ & $14,082.6$ & $\mid 13,609.7$ & $\mid 13,300.8$ & $14,297.1$ & $\mid 12,433.1$ & $15,270.6$ & $13,356.3$ & $12,415.7$ & $10,103.6$ & 12,0 & 8.5 & 4.4 \\
\hline & $2,618.4$ & $8,393.4$ & $8,124,9$ & 233.1 & $6,914.2$ & $6,940.2$ & $8,494.5$ & $6,998.1$ & $7,563.8$ & $7,651.5$ & ;608.3 & $8,432.7$ & $8,149.5$ & $7,964.5$ & $8,561.1$ & $7,445.0$ & $9,144.1$ & $7,997.8$ & $7,434.5$ & 050.0 & 3 & 280.6 & 77.3 \\
\hline $\mathbf{P} \mathbf{P}_{\mathbf{T}}$ & $\mathrm{t}$ & 2.72 & 2. & & 66 & 2. & & & 2 & 2. & & 2.65 & 2.66 & .66 & 2.68 & 2 & 43 & 2.57 & 2. & 2. & & 2.6 & 2.5 \\
\hline $\mathrm{PE}$ & 4.00 & 4.54 & 4.44 & 4.32 & 4.44 & 40 & 4.49| & 4.50 & 4.33 & 4.40 & 4.29 & 4.42 & 4.44 & 4.49 & 4.48 & 4.28 & 06 & 29 & 4.29 & 4.28 & .18 & 4.40 & 4.32 \\
\hline 1 Cons & & & 46 & 37 & 31 & & . & 59 & 40 & 27 & re & 20 & & ent & 31 & & $\sqrt{2}$ & $\sqrt{4}$ & 24 & ${ }^{49}$ & 40 & 73.00 & 41.00 \\
\hline & & & 1.22 & 1.18 & 11 & 91 & & & $\cdots$ & 1.09 & .24 & & & 1.07 & 1.09 & 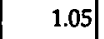 & .07 & 1.06 & 1.03 & 1.05 & .07 & 8.14 & 1.0 \\
\hline & 135 & 555 & 8,026 & ,037 & 839 & 5,019 & 558 & ,192 & $; 543$ & 3,070 & 9,152 & 1775 & 7,811 & 3,173 & 3,898 & 4,073 & 8,723 & 3,837 & 8,401 & 2,633 & 90 & & \\
\hline & & 789 & 2.598 & 17,954 & 3,443 & 8,803 & 3,698 & 38,553 & 43,119 & 8,746 & 3,099 & 59,744 & 6,700 & 1,769 & 7,084 & 82,735 & 8,324 & 4,353 & 9,450 & 04,39 & 09,614 & & \\
\hline
\end{tabular}

1586

Diesel Control

Before 1/98 After 1/98

\begin{tabular}{|c|c|c|c|c|c|c|c|c|c|c|c|c|c|c|c|c|c|c|c|c|c|c|c|}
\hline 1586 & Diesel 0 & ontrol & & & & & & & & & & & & & & & & & & & & Before $1 / 98$ & After Y/98 \\
\hline & Apr-97 & May-97 & Jun-97 & Jul-97 & Aug-97 & Sep-97 & Oet-97 & Nov-97 & Dec-97 & Jan-98 & Feb-98 & Mar-98 & Apr-98 & May-98 & Jun-98 & Jul-98 & Aug-98 & Sep-98 & Oct-98 & Nov-98 & Dec-98 & Total & Total \\
\hline Total Mileage & & & 3,971 & 6,627 & 6,089 & 5,515 & 6,398 & 5,313 & 7,173 & 6,891 & 5,555 & 5,272 & 7,184 & 9,428 & 9,026 & 9,665 & 6,112 & 6,531 & 6,760 & 5,619 & 5,445 & 41,086 & 83,488 \\
\hline Fuel Mileage Base & & & 3,971 & 6,627 & 6,089 & 4,254 & 6,036 & 5,313 & 7,173 & 6,891 & 4,660 & 5,272 & 7,184 & 8,001 & 7,323 & 8,699 & 6,112 & 5,834 & 5,400 & 5,619 & 4,178 & 39,463 & 75,173 \\
\hline Fuel (D2) & & & 563.1 & 924.4 & 859.1] & 599.8 & 839.7 & 761.7 & 1031.8 & 1075.2 & 680.8 & 747.3 & 1001.3 & 1117.3 & 982.1 & 1212.4 & 837.4 & 807.5 & 774.6 & 869.1 & 597.8 & 5579.5 & 10702.7 \\
\hline MPG & & & 7.05 & 7.17 & 7.09 & 7.09 & 7.19 & 6.98 & 6.95 & 6.41 & 6.85 & 7.05 & 7.17 & 7.16 & 7.46 & 7.18 & 7.30 & 7.22 & 6.97 & 6.47 & 6.99 & 7.07 & 7.02 \\
\hline il Consumption & & & & 3.00 & & & 6.00 & & & & & & & & & & & & & & & & \\
\hline 1 Qts per 1000 & & & 153189 & $759816]$ & 1905] & 74201 & 13818 & $19,131\}$ & 263041 & 33195 & 38.750 & 44022 & 55061 & 6034 & $69660 \mathrm{~L}$ & 793351 & 85437 & 01968 & 98778 & 04347 & $10970 ?$ & 26304 & 1097.792 \\
\hline d Odometer & & & 103,109 & 159,010 & 1,900 & 7,4201 & {$[0,010$} & $19, \mid 31\}$ & $20, \times 04_{1}$ & $3,3_{1}, 195$ & 38,750, & & 51,2000 & $80,6344]$ & 69,600 & 79,250 & 85,431 & $91,908]$ & 98,288 & 104,347 ] & 109,294 & 26,304 & $109,1,92$ \\
\hline
\end{tabular}
End Odometer

Diesel Control

\begin{tabular}{|c|c|c|c|c|c|c|c|c|c|c|c|c|c|c|c|c|c|c|c|c|c|c|c|}
\hline 1592 & Diesel C & ontrol & & & & & & & & & & & & & & & & & & & & Before 1/98 & After $1 / 98$ \\
\hline & Apr-97 & May-97 & \begin{tabular}{|l|} 
Jun-97 \\
\end{tabular} & Jul-97 & Aug-97 & Sep-97 & Oct-97| & Nov-97 & Dec-97| & Jan-98 & Feb-98 & \begin{tabular}{|l|} 
Mar-98 \\
\end{tabular} & Apr-98 & \begin{tabular}{|l|} 
May-98 \\
\end{tabular} & \begin{tabular}{|l|} 
Jun-98 \\
\end{tabular} & Jul-98 & Aug-98 & Sep-98 & Oct-98 & \begin{tabular}{|l|} 
Nov-98 \\
\end{tabular} & Dec-98 & Total & Total \\
\hline Total Mileage & & & 4,740 & 6,521 & 5,342 & 6,036 & 6,472 & 5,199 & 5,850 & 5,665 & 5,126 & 5,333 & 6,085 & 5,825 & 6,521 & 6,393 & 5,704 & 4,682 & 5,221 & 4,247 & 4,087 & 40,160 & 64,889 \\
\hline Base & & & 4,740 & 6,521 & 5,342 & 5,302 & 6,271 & 4,730 & 5,850 & 4,709 & 4,243 & 4,530 & 6,085 & 5,403 & 5,318 & 6,393 & 5,704 & 4,682 & 4,560 & 3,304 & 3,623 & 88,756 & 58,554 \\
\hline uel (D2) & & & 674.1 & 900.2 & 771.0 & 721.3 & 878.2 & 661.2 & 812.8 & 663.2 & 606.3 & 643.4 & 867.8 & 750.7 & 761.2 & 924.0 & 825.0 & 687.7 & 657.7 & 462.5 & 534.5 & 5418.8 & 8383.8 \\
\hline & & & 7.03 & 7.24 & 6.93 & 7.35 & 7.14 & 7.15 & 7.20 & 7.10 & 7.00 & 7.04 & 7.01 & 7.20 & 6.99 & 6.92 & 6.91 & 6.81 & 6.93 & 7.14 & 6.78 & 7.15 & 6.98 \\
\hline Oil Consumption & & & & 4.00 & 3.00 & & & & & & & & & & & & & & & & & & \\
\hline Oil Qts per 1000 & & & & & & & & & & & & & & & & & & & & & & & \\
\hline nd Odometer & & & 125,202 & 131,723 & 137,065 & 143,101 & 49,573 & .766 & 8,616 & 14,281 & 19,407 & 24,740 & 30,825 & 36,650 & 43,171 & 9,564 & 35,268 & 59,950 & 65,171 & 69,418 & 73,505 & 8,616 & 73,505 \\
\hline
\end{tabular}




\begin{tabular}{|c|c|c|c|c|c|c|c|c|c|c|c|c|c|c|c|c|c|c|c|c|c|c|c|}
\hline & Apr-97 & May-97 & Jun-97| & Jul-97| & Aug-97 & Sep-97| & Oct-97| & Nov-97| & Dec-97 & Jan-98 & Feb-98 & Mar-98 & Apr-98 & May-98 & Jun-98 & Jul-98 & Aug-98 & Sep-98 & Oct-98 & Nov-98 & Dec-98 & Total & er 1/98 \\
\hline Cotal Mileage & & & 4,405 & 6,525 & 6,206 & 5,943 & 5,789 & 6,298 & 5,663 & 4,700 & 4,770 & 5,464 & 6,738 & 7,409 & 7,789 & 7,621 & 7,035 & 6,163 & 4,769 & 5,716 & 6,012 & 0,829 & 74,18 \\
\hline uel Mileage Base & & & 4,405 & 5,384 & 6,206 & 4,572 & 5,789 & 6,298 & 5,663 & 4,233 & 4,770 & 3,922 & 6,186 & 7,409 & 7,187 & 6,044 & 6,164 & 6,163 & 4,485 & 5,122 & Dיم49 & 8,317 & \\
\hline sel (D2) & & & 619.5 & 798.0 & 929.5 & 656.4 & 852.7 & 917.3 & 827.6 & 637.9 & 701.3 & 588.8 & 854.0 & 1026.2 & 1008.0 & 878.7 & 867.6 & 833.1 & 610.4 & 763.7 & 774.1 & 8001.0 & 54 \\
\hline PG & & & 7.11 & 6.75 & 6.68 & 6.97 & 6.79 & 6.87 & 6.84 & 6.64 & 6.80 & 6.66 & 7.24 & 7.22 & 7.13 & 6.88 & 7.10 & 7.40 & 7.35 & 6.71 & 7.17 & 6.84 & \\
\hline $\begin{array}{l}\text { I Cons } \\
\text { I Qts }\end{array}$ & & & & & & & & & & & & & & & & & & & & & & & \\
\hline Id Odomeler & & & 159,251 & 165,776 & 918 & 6,861 & 12,650 & 18,948 & 24,611 & 29,311 & 34,081 & 39,545 & 46,283 & 53,692 & 61,481 & 69,102 & 76,137 & 82,300 & 37,06 & 2,78 & 8,79 & 4,611 & \\
\hline
\end{tabular}

LNG Truck

\begin{tabular}{|c|c|c|c|c|c|c|c|c|c|c|c|c|c|c|c|c|c|c|c|c|c|c|c|}
\hline 01 & LNG Tru & & & & & & & & & & & & & & & & & & & & & Ber & $\mathbf{S}$ \\
\hline & Apr-97 & May-97 & Jun-97 & Jul-97 & Aug-97 & Sep-97 & Oct-97 & Nov-97 & Dec-97 & Jan-98 & Feb-98 & Mar-98 & Apr-98 & May-98 & Jun-98 & Jul-98 & Aug-98 & Sep-98 & Oct-98 & Nov-98 & Dec-98 & Total & Total \\
\hline otal Mileage & 1,293 & 4,421 & 4,643 & 3,138 & 4,033 & 4,053 & 4,328 & 3,430 & 3,902 & 2,031 & 2,648 & 3,634 & 3,115 & 5,416 & 4,880 & 5,303 & 4,101 & 3,017 & 4,597 & 4,102 & 3,206 & 33,241 & 46,050 \\
\hline el N & 1,293 & 4,421 & 643 & 3,138 & 4,033 & 4,053 & 4,328 & 3,430 & 3,902 & 2,031 & 648 & 3,634 & 2,737 & 4,887 & 4,880 & 4,591 & 3,825 & 3,017 & 4,597 & 3,564 & 3,206 & 33,241 & 33,616 \\
\hline el 0 & 2317.14 & 5834.42 & 6128.94 & 4275.81 & 5065.76 & 5086.03 & 5692.51 & 4245.62 & 5129.62 & 2889.57 & 3350.02 & 4868.30 & 3492.95 & 6294.00 & 6243.91 & 6294.38 & 5740.28 & 4465.12 & 5815.45 & 4798.21 & 3377.27 & 3775.85 & 7629.46 \\
\hline NG & 656.4 & $1,652.8$ & 36.2 & $1,211.3$ & $1,435.1$ & $1,440.8$ & $1,612.6$ & $1,202.7$ & 1, & 6 & .0 & $1,379.1$ & 989.5 & & 68.8 & 3.1 & 626.1 & 264.9 & $1,710.4$ & 9.3 & $1,262.8$ & & $16,694.6$ \\
\hline el (c & 393.1 & 989.7 & 039.7 & 725.3 & 9.3 & 862.8 & 5.6 & 720.2 & 870.1 & 490 & 68.3 & 825.8 & 592.5 & ,067.7 & 059.2 & ,067.7 & 973.7 & 757.4 & ,024.2 & 813.9 & 756.1 & 25.8 & 9,996 \\
\hline PG & 1.97 & 2.67 & 2.67 & 59 & 2.81 & 2.8 & 2.68 & D. & 2. & 2. & & 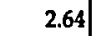 & 2.77 & 2.74 & 2.7 & 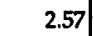 & 2.3 & 2.3 & 2.69 & $2.6 ?$ & 2.5 & 2.6 & 2.61 \\
\hline PEG & 3.29 & 4.47 & 4.47 & 4.33 & 4.69 & 4.70 & 4.48 & 4.76 & 4.48 & 4.14 & 4.66 & 4.40 & 4.62 & 4.58 & 4.61 & 4.30 & 3.93 & 3.98 & 4.49 & 4.38 & 4.24 & 4.4 & 4.3 \\
\hline & & & 4.00 & 8.00 & 4.00 & 2.00 & 12.00 & 8.00 & 8.00 & 0.00 & $4 . C$ & 4.00 & 0 & 10.00 & 12.00 & nl & 12.00 & 0. & $\infty$ & 4.00 & 4.00 & 46.00 & 65.00 \\
\hline & & & 0.86 & 1.54 & 1.35 & 1.13 & 1.49 & 1.61 & 1.67 & & & 1.51 & 39 & & 1.54 & & & 1.00 & | & & & 1.67 & 1.41 \\
\hline id Odometer & 1,365 & 5,786 & 10,429 & 13,567 & 7,600 & 21,653 & ;,981 & 9,411 & 33,313 & 15,344 & 7,992 & 1,626 & 4,741 & 50,157 & 5,037 & 30,340 & 4,441 & 7,458 & 2,055 & 6,157 & 79,363 & 33,313 & 9,36 \\
\hline
\end{tabular}

\begin{tabular}{|c|c|c|c|c|c|c|c|c|c|c|c|c|c|c|c|c|c|c|c|c|c|c|c|}
\hline 32 & LNG Tru & & & & & & & & & & & & & & & & & & & & & Before 1/98 & Since $1 / 98$ \\
\hline & Apr-97 & May-97 & $n-97$ & \begin{tabular}{|l|l|} 
Jul-97 \\
\end{tabular} & ig-97 & ep-97 & ct-97) & Nov-97 & Dec-97 & Jan-98 & Feb-98 & Mat-98 & pr-98 & May-98 & Jun-98 & Jul-98 & ug-98 & Sep-98 & ct-98 & ov-98 & Dec-98 & tal & Total \\
\hline Cotal M & 288 & 443 & 4,580 & 508 & 738 & 3,429 & 4,757 & 2,572 & ,8995 & 4,400 & 3,234 & 3,415 & 5,401 & 6,085 & 4,663 & \begin{tabular}{|l|}
5,003 \\
\end{tabular} & 5,158 & 5,653 & 4,910 & 3,515 & 4,396 & .210 & 5,833 \\
\hline & 1,288 & 4,443 & & & & & 5 & & 395 & & & & & & 3 & & 58 & 92 & 4,435 & & 396 & & 1,552 \\
\hline & 791.81 & 5702.89 & 6078.60 & 4979.38 & 5115.89 & 5036.15 & 6205.35 & 3791.98 & 5478.01 & 6055.07 & 3932.73 & 4809.37 & 6458.01 & 7365.47 & 5921.75 & 4432.07 & 7038.76 & 6413.88 & 5134.26 & 4128.84 & 4580.50 & .06 & 66270.71 \\
\hline & 507.6 & $1,615.5$ & 2.0 & 10.6 & 99.3 & $1,426.7$ & & 1.2 & 8 & & & & & & & .5 & & & & & & & 19,28 \\
\hline wel & 303.9 & 967.4 & 031.1 & 844.7 & 867.8 & 854.3 &, 052.6 & 64 & 929.2 & |027.1 & 667 & 815.8 & 095.5 & 249.4 & 004.5 & 751.8 & 8.0 & 08 & 1.4 & 700.4 & 71.4 & 4.4 & $1,546.5$ \\
\hline APG & 2.54 & 2.75 & 2.66 & 2.4 & & & 2.52 & & 1 & 7 & & & 67 & 2. & $2.78 \mid$ & 2.73 & 2. & & & & .71 & 55 [ & 2.6 \\
\hline APEG & 4.24 & 4.59 & 4.44 & 4.15 & 4.31 & 4.01 & 4.21 & 4.00 & 19 & 4.28 & 4.36 & 4.19 & 45 & 4.66 & 4.64 & 4.56 & 4.32 & 59 & 4.52 & & 4.53 & .25 & 3. \\
\hline & & & 6.00 & 0.00 & 2.00 & 0.00 & 2.00 & 8.00 & 3.00 & 4.00 & 4.00 & 0.00 & 0.00 & 2.00 & 11.00 & 2.00 & $13 . c$ & 6.00 & 6.00 & 0 & 5.00 & 1.00 & .0 \\
\hline & & & 1.31 & 0.74 & 0.68 & 0.5 & 0.50 & 0.80 & & & 0.85 & 1 & 0.68 & 0.63 & 0.78 & 0.75 & & & 0.93 & & 0.95 & .179 & 1.02 \\
\hline nd Od & 1,413 & 5,856 & 10,436 & 3,944 & 682 & ,111 & 5,868 & 28,440 & .335 & 6,735 & 39,969 & 43,384 & 48,785 & 54,870 & 59,533 & 4,536 & 69,694 & 75,347 & 0,257 & 3,772 & 8,168 & 2,335 & 8,16 \\
\hline
\end{tabular}

\begin{tabular}{|c|c|c|c|c|c|c|c|c|c|c|c|c|c|c|c|c|c|c|c|c|c|c|c|}
\hline 03 & NG & & & & & & & & & & & & & & & & & & & & & Before $1 / 98$ & Since 1/98 \\
\hline & Apr-97 & \begin{tabular}{|l|} 
May-977 \\
\end{tabular} & Jun-97 & JuI-97 & Aug-97 & ep-97 & ct-97| & $\begin{array}{l}\text { Nov-97 } \\
\end{array}$ & \begin{tabular}{|l|} 
Dec-97| \\
\end{tabular} & in-98 & Feb-98 & Mar-98 & pr-98 & May-98 & Jun-98 & Jul-98 & Aug-98 & Sep-98 & Oct-98 & Ov-98 & Dec-98 & Total & Total \\
\hline 5 & 228 & 941 & 888 & 936 & 816 & & 6,020 & 4356 & 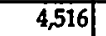 & 5,650 & 3,858 & 5,398 & 6,083 & 5,878 & 6,264 & 4,404 & 4,718 & 2,657 & 3,778 & 320 & 1,834 & & 50,842 \\
\hline & 1,228 & 941[ & 4,521 & 48 & 316 & ,58 & 5,738 & 4,356 & 4,188 & 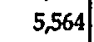 & 8. & 5,398 & 8 & 4,730 & 5,470 & 97 ] & 8 & 57 & 75 & 20 & 34 & & 355 \\
\hline Hel ( & 169.01 & 6547.27 & 5933.27 & 5874.85 & 4245.85 & 5121.01 & 7596.92 & 6112.00 & 5685.77 & 7457.16 & 5468.47 & 7370.92 & 7386.43 & 6756.54 & 7413.35 & 5116.47 & 7441.50 & 3674.37 & 47.24 & 38.19 & 2717.86 & 95 & 88 \\
\hline VC & 614.5 & $1,854.8$ & 680.8 & $1,664.3$ & $1,202.8$ & 450.7 & 152.1 & $1,731.4$ & $1,610.7$ & ,112.5 & $1,549.1$ & $2,088.1$ & $2,092.5$ & 14.0 & $2,100.1$ & 449.4 & 8.1 & $1,040.9$ & 67.6 & 2.5 & 7.4 & & $8,812.2$ \\
\hline uel (D2 EQ) & 367.9 & 10.6 & 06.5 & 6.6 & & 8.7 & 288.7 & 036.8 & 964.5 & 0 & 927.6 & 0.3 & 253.0 & 6.1 & 257.5 & 67.9 & 2.3 & 623.3 & 8.9 & & 501.4 & 60.5] & 264.8 \\
\hline & & 2.66 & & & & & & & & & & & & & & 2.69 & & & & & 2.19 & 57. & 25 \\
\hline & 3 & 4.45 & & & & & & & 4. & & & & & & & 4.49 & & & & & & 0 & 4.20 \\
\hline il & & & 3.00 & 10.00 & 6.00 & 2. & 16.00 & 2.00 & 12.00 & 0 & 5.00 & 4.0 & & 3.00 & 4. & 2.00 & .00 & & & & 0.00 & .00 & 0.00 \\
\hline illo & & & 0.61 & 1.32 & 1.50 & 1.29 & 56 & 1.84 & 1.96 & 1.85 & 2.04 & 1.89 & 1.82 & 1.69 & 1.59 & 1.51 & 1.51 & 1.46 & 1.39 & 1.39 & 1.35 & 1.96 & 0.9 \\
\hline a Odometer & 1,318 & 6,259 & 11,147 & 16,083 & 18,899 & 22,480 & 28,500 & 32,856 & 37,372 & 43,022 & 46,880 & 52,278 & 58,361 & 64,239 & 70,503 & 74,907 & 79,625 & 82,282 & 86,060 & 6,360 & 38,214 & 7,372 & 8,21 \\
\hline
\end{tabular}




\begin{tabular}{|c|c|c|c|c|c|c|c|c|c|c|c|c|c|c|c|c|c|c|c|c|c|c|c|}
\hline & & & & & & & & & & & & & & & & & & & & & & e 1/98 & iee \\
\hline & Apr-97 & May-97 & Jun-97 & Jul-97 & Aug-97 & Sep-97| & Oct-97 & Nov-97 & Dec-97 & Jan-98 & Feb-98 & Mar-98 & Apr-98 & May-98 & Jun-98 & Jul-98 & Aug-98 & Sep-98 & Oct-98) & Nov-98 & Dec-98 & Total & Total \\
\hline Total Mileage & 1,212 & 5,574 & 5,706 & 5,356 & 5,489 & 5,360 & 3,424 & 4,397 & 5,410 & 5,767 & 4,028 & 7,916 & 6,956 & 5,069 & 5,315 & 5,651 & 5,589 & 6,029 & 5,097 & 4,946 & 5,218 & 41,928 & 67,581 \\
\hline el Mileage Base & 1,212 & 5,574 & 5,706 & 5,356 & 5,021 & 4,612 & 3,424 & 3,771 & 5,410 & 5,506 & 3,144 & 7,044 & 6,525 & 4,114 & 5,315 & 4,609 & 5,200 & 6,029 & 4,035 & 4,314 & 4,581 & 40,085 & 60,416 \\
\hline el (LNG, lbs) & 1672.28 & 6864.05 & 7263.21 & 6686.60 & 6137.01 & 5544.54 & 4595.95 & 5131.85 & 7067.79 & 7307.87 & 4347.02 & 8989.26 & 8772.23 & 5344.07 & 6856.31 & 6400.10 & 7410.99 & 8026.15 & 4924.18 & 6058.42 & 5117.29 & 50963.28 & 79553.89 \\
\hline NG & 473.7 & $1,944.5$ & $2,057.6$ & $1,894.2$ & $1,738.5$ & $1,570.7$ & $1,302.0$ & $1,453.8$ & $2,002.2$ & $2,070.2$ & $1,231.5$ & $2,546.5$ & $2,485.1$ & $1,513.9$ & 942.3 & ,813.1 & $2,099.4$ & $2,273.7$ & $1,505.0$ & $1,716.3$ & $1,811.9$ & 37.2 & $23,008.8$ \\
\hline Fuel (D2 EQ) & 283.7 & $1,164.4$ & $1,232.1$ & $1,134.3$ & $1,041.0$ & 940.5 & 779.6 & 870.5 & $1,198.9$ & $1,239.7$ & 737.4 & $1,524.9$ & $1,488.1$ & 906.5 & $1,163.1$ & , 085.7 & $1,257.1$ & $1,361.5$ & 901.2 & $1,027.7$ & $1,085.0$ & 645.0 & $13,777,7$ \\
\hline MPG & 2.56 & 2.87 & 2.77 & 2.83 & 2.89 & 2.94 & 2.63 & 2.59 & 2.70 & 2.66 & 2.55 & 2.77 & 2.63 & 2.72 & 2.74 & 2.54 & 2.48 & 2.65 & 2.68 & 2.51 & 2.53 & 2.78 & 2.63 \\
\hline $\mathbf{G}$ & 4.27 & 4.79 & 4.63 & 4.72 & 4.82 & 4.90 & 4.39 & 4.33 & 4.51 & 4.44 & 4.26 & 4.62 & 4.39 & 4.54 & 4.57 & 4.25 & 4.14 & 4.43 & 4.48 & 4.20 & 4.22 & 4.64 & 4.39 \\
\hline & & & 9.00 & 3.00 & 2.00 & 2.00 & 0.00 & 5.00 & 6.00 & 4.00 & 8.00 & 0.00 & 8.00 & 7.00 & 4.00 & 0.00 & 8.00 & 8.00 & 7.00 & 7.00 & 4.00 & 27.00 & 65.00 \\
\hline & & & 1.58 & 1.08 & 0.85 & 0.73 & 0.63 & 0.71 & 0.77 & 0.76 & 0.87 & 0.74 & 0.79 & 0.83 & 0.83 & 0.76 & 0.81 & 0.85 & 0.88 & 0.90 & 0.90 & 0.77 & 0.96 \\
\hline End Odometer & 1,318 & 6,892 & 12,598 & 17,954 & 23,443 & 28,803 & 32,227 & 36,624 & 42,034 & 47,800 & 51,828 & 59,744 & 66,700 & 71,769 & 77,084 & 82,735 & 88,324 & 94,353 & 99,450 & 104,396 & 109,614 & 42,034 & 109,614 \\
\hline
\end{tabular}

\begin{tabular}{|c|c|c|c|c|c|c|c|c|c|c|c|c|c|c|c|c|c|c|c|c|c|c|c|}
\hline 1505 & LNG Tru & & & & & & & & & & & & & & & & & & & & & Before $1 / 98$ & Since $1 / 98$ \\
\hline & Apr-97 & May-97 & Jun-97 & Jul-97 & Aug-97 & Sep-97 & Oct-97 & Nov-97 & Dec-97 & Jan-98 & Feb-98 & Mar-98 & Apr-98 & May-98 & Jun-98 & Jul-98 & Aug-98 & Sep-98 & Oct-98 & \begin{tabular}{|l|} 
Nov-98 \\
\end{tabular} & Dec-98 & Total & Total \\
\hline Total Mileage & 789 & 3,420 & 3,471 & 3,011 & 1,802 & 2,180 & 4,539 & 3,634 & 2,351 & 2,527 & 1,082 & 4,023 & 4,636 & 5,362 & 5,725 & 5,175 & 4,650 & 5,114 & 4,564 & 4,232 & 5,244 & 25,197 & 52,334 \\
\hline Fuel Mileage Base & 789 & 3,420 & 3,471 & 3,011 & 1,802 & 2,180 & 4,539 & 3,634 & 2,351 & 2,294 & 1,082 & 3,687 & 4,214 & 4,639 & 5,012 & 4,543 & 4,650 & 5,114 & 3,945 & 3,642 & 5,244 & 25,197 & 48,066 \\
\hline Fuel (LNG, lbs) & |1189.74 & 4846.85 & 4897.27 & 4420.74 & 2609.19 & 3337.10 & 6122.30 & 4958.20 & 3487.58 & 2977.79 & 1669.98 & 4970.32 & 5771.07 & 6213.11 & 6488.65 & 6162.92 & 6950.65 & 7127.20 & 4946.03 & 5103.13 & 6579.01 & 35868.97 & 64959.86 \\
\hline LNG std gal & 337.0 & $1,373.0$ & $1,387.3$ & $1,252.3$ & 739.1 & 945.4 & $1,734.4$ & $1,404.6$ & 988.0 & 843.6 & 473.1 & $1,408.0$ & $1,634.9$ & $1,760.1$ & $1,838.1$ & $1,745.9$ & $1,969.0$ & $2,019.0$ & $1,585.1$ & $1,445.6$ & $2,184.5$ & $10,161.2$ & $18,906.9$ \\
\hline Fuel (D2 EQ) & 201.8 & 822.2 & 830.7 & 749.9 & 442.6 & 566.1 & $1,038.5$ & 841.1 & 591.6 & 505.1 & 283.3 & 843.1 & 979.0 & $1,053.9$ & $1,100.7$ & $1,045.4$ & $1,179.1$ & $1,209.0$ & 949.1 & 865.7 & $1,308.1$ & $6,084.5$ & $11,321.5$ \\
\hline MPG & 2.34 & 2.49 & 2.50 & 2.40 & 2.44 & 2.31 & 2.62 & 2.59 & 2.38 & 2.72 & 2.29 & 2.62 & 2.58 & 2.64 & 2.73 & 2.60 & 2.36 & 2.53 & 2.49 & 2.52 & 2.40 & 2.48 & 2.54 \\
\hline MPEG & 3.91 & 4.16 & 4.18 & 4.02 & 4.07 & 3.85 & 4.37 & 4.32 & 3.97 & 4.54 & 3.82 & 4.37 & 4.30 & 4.40 & 4.55 & 4.35 & 3.94 & 4.23 & 4.16 & 4.21 & 4.01 & 4.14 & 4.25 \\
\hline Oil Consumption & & & 4.00 & 4.00 & 4.00 & 0.00 & 0.00 & 7.00 & 7.00 & 0.00 & 4.00 & 4.00 & 0.00 & 5.00 & 8.00 & 0.00 & 4.00 & 4.00 & 0.00 & 8.00 & 9.00 & 26.00 & 46.00 \\
\hline Oil Qts per 1000 & & & 1.15 & 1.23 & 1.45 & 1.15 & 0.80 & 1.02 & 1.24 & 1.11 & 1.22 & 1.19 & 1.02 & 1.01 & 1.06 & 0.95 & 0.94 & 0.93 & 0.86 & 0.93 & 0.98 & 1.24 & 0.88 \\
\hline End Odometer & 1,135 & 4,555 & 8,026 & 11,037 & 12,839 & 15,019 & 19,558 & 23,192 & 25,543 & 28,070 & 29,152 & 33,175 & 37,811 & 43,173 & 48,898 & 54,073 & 58,723 & 63,837 & 68,401 & 72,633 & 77,877 & 25,543 & 77,877 \\
\hline
\end{tabular}
End Odometer

LNG Truck

Before 198 Since $1 / 98$

\begin{tabular}{|c|c|c|c|c|c|c|c|c|c|c|c|c|c|c|c|c|c|c|c|c|c|c|c|}
\hline 506 & LNG Tr & & & & & & & & & & & & & & & & & & & & & Before $1 / 98$ & $\operatorname{Sin}$ \\
\hline & Apr-97 & May-97 & Jun-97 & Jul-97 & Aug-97 & Sep-97 & Oct-97 & Nov-97 & Dec-97 & Jan-98 & Feb-98 & Mar-98 & Apr-98 & May-98 & Jun-98 & Jul-98 & Aug-98 & Sep-98 & Oct-98 & Nov-98 & Dec-98 & Total & Total \\
\hline Total Mileage & 1,316 & 4,612 & 4,986 & 4,705 & 4,189 & 5,106 & 4,682 & 2,968 & 3,600 & 4,545 & 4,351 & 5,640 & 5,520 & 4,625 & 4,188 & 4,247 & 3,891 & 3,350 & 4,144 & 4,240 & 3,719 & 36,164 & 52,460 \\
\hline Fuel Mileage Base & 1,316 & 4,612 & 4,864 & 4,481 & 3,922 & 4,245 & 4,334 & 2,968 & 3,600 & 4,096 & 3,657 & 4,682 & 4,515 & 4,344 & 4,188 & 4,247 & 3,891 & 3,350 & 4,038 & 3,719 & 3,719 & 34,342 & 48,446 \\
\hline uel (LNG, Ibs) & 1944.81 & 6337.43 & 6571.15 & 5988.37 & 5351.12 & 5641.83 & 5548.69 & 4203.62 & 5257.91 & 5638.76 & 5124.23 & 6233.59 & 6217.05 & 6151.74 & 6068.54 & 6039.42 & 5769.93 & 4675.76 & 5027.63 & 5059.24 & 4461.90 & 46844.93 & 66467.79 \\
\hline LNG std gal & 550.9 & $1,795.3$ & $1,861.5$ & $1,696.4$ & $1,515.9$ & $1,598.3$ & $1,571.9$ & $1,190.8$ & $1,489.5$ & $1,597.4$ & $1,451.6$ & $1,765.9$ & $1,761.2$ & $1,742.7$ & $1,719.1$ & $1,710.9$ & $1,634.5$ & $1,324.6$ & $1,558.0$ & $1,433.2$ & $1,452.5$ & 70.5 & $19,151.7$ \\
\hline Fuel (D? & 329.9 & $1,075.0$ & $1,114.7$ & $1,015.8$ & 907.7 & 957.0 & 941.2 & 713.1 & 891.9 & 956.5 & 869.2 & $1,057.4$ & $1,054.6$ & $1,043.5$ & $1,029.4$ & $1,024.5$ & 978.8 & 793.2 & 932.9 & 858.2 & 869.8 & , 946.4 & $11,468.1$ \\
\hline IPG & 2.39 & 2.57 & 2.61 & 2.64 & 2.59 & 2.66 & 76 & 2.49 & 2.42 & 2.56 & 2.52 & 2.65 & 2.56 & 2.49 & 2.44 & 2.48 & 2.38 & 2.53 & 2.59 & 2.59 & 2.56 & 2.59 & 2.53 \\
\hline MPEG & 3.99 & 4.29 & 4.36 & 4.41 & 4.32 & 4.44 & 4.60 & 4.16 & 4.04 & 4.28 & 4.21 & 4.43 & 4.28 & 4.16 & 4.07 & 4.15 & 3.98 & 4.22 & 4.33 & 4.33 & 4.28 & 4.32 & 4.22 \\
\hline & & & 10.00 & 2.00 & 4.00 & 2.00 & 0.00 & 2.00 & 0.00 & 0.00 & 12.00 & 0.00 & 0.00 & 4.00 & 2.00 & 0.00 & 4.00 & 0.00 & 0.00 & 5.00 & 9.00 & 20.00 & 36.00 \\
\hline & & & 2.01 & 1.24 & 1.15 & 0.95 & 0.76 & 0.75 & 0.66 & 0.58 & 0.82 & 0.71 & 0.64 & 0.66 & 0.64 & 0.60 & 0.62 & 0.59 & 0.56 & 0.60 & 0.68 & 0.66 & 0.69 \\
\hline End Odometer & 1,423 & 6,035 & 11,021 & 15,726 & 19,915 & 25,021 & 29,703 & 32,671 & 36,271 & 40,816 & 45,167 & 50,807 & 56,327 & 60,952 & 65,140 & 69,387 & 73,278 & 76,628 & 80,772 & 85,012 & 88,731 & 36,271 & 88,731 \\
\hline
\end{tabular}

\begin{tabular}{|c|c|c|c|c|c|c|c|c|c|c|c|c|c|c|c|c|c|c|c|c|c|c|c|}
\hline 1507 & LNG Tru & & & & & & & & & & & & & & & & & & & & & Before $1 / 98$ & Since $1 / 98$ \\
\hline & Apr-97 & May-97| & Jun-97 & Jul-97 & Aug-97 & Sep-97 & Oct-97) & Nov-97 & Dec-97 & Jan-98 & Feb-98 & Mar-98 & Apr -98 & May-98 & Jun-98 & Jul- 98 & Aug-98 & Sep-98 & Oct-98 & Nov-98 & Dec-98 & Total & Total \\
\hline Total Mileage & 1,342 & 5,372 & 4,816 & 3,641 & 4,570 & 2,975 & 5,538 & 5,508 & 5,485 & 4,147 & 3,555 & 5,053 & 4,777 & 4,791 & 5,511 & 4,378 & 5,029 & 5,583 & 5,265 & 4,098 & 3,668 & 39,247 & 55,855 \\
\hline Fuel Mileage Base & 1,342 & 5,372 & 4,096 & 3,248 & 4,516 & 2,975 & 5,538 & 4,920 & 5,143 & 4,147 & 3,525 & 4,689 & 4,378 & 4,523 & 4,572 & 4,005 & 5,029 & 5,151 & 4,651 & 3,645 & 3,668 & 37,150 & 51,982 \\
\hline Fuel (LNG, Ibs) & \begin{tabular}{|l|} 
\\
$\mid 1717.97$ \\
\end{tabular} & 6640.18 & 5676.49 & 4614.91 & 5963.29 & 4016.31 & 7016.81 & 6312.70 & 6805.77 & 5651.81 & 4647,42 & 6330.28 & 5728.22 & 5887.22 & 6008.60 & 5847.22 & \begin{tabular}{|l|}
7315.10 \\
\end{tabular} & 7323.64 & 5523.92 & 5169.40 & 4121.79 & 48764.43 & 69554.62 \\
\hline LNG std gal & 486.7 & $1,881.1$ & $1,608.1$ & $1,307.3$ & $1,689.3$ & $1,137.8$ & $1,987.8$ & $1,788.3$ & $1,928.0$ & $1,601.1$ & $1,316.5$ & $1,793.3$ & $1,622.7$ & $1,667.8$ & $1,702.2$ & $1,656.4$ & $2,072.3$ & $2,074.7$ & $1,820.5$ & $1,464.4$ & $1,492,2$ & $13,814.3$ & $20,284.0$ \\
\hline Fuel (D2 EQ) & 291.4 & $1,126.4$ & 962.9 & 782.8 & $1,011.6$ & 681.3 & $1,190.3$ & $1,070.8$ & $1,154.5$ & 958.7 & 788.4 & $1,073.8$ & 971.7 & 998.7 & \begin{tabular}{|l|}
$1,019.3$ \\
\end{tabular} & 991.9 & $1,240.9$ & $1,242.3$ & $1,090.1$ & 876.9 & 893.5 & $8,272.0$ & $12,146.1$ \\
\hline MPG & 2.76 & 2.86 & 2.55 & 2.48 & 2.67 & 2.61 & 2.79 & 2.75 & 2.67 & 2.59 & 2.68 & 2.61 & 2.70 & 2.71 & 2.69 & 2.42 & 2.43 & 2.48 & 2.55 & 2.49 & 2.46 & 2.69 & 2.56 \\
\hline MPEG & 4.60 & 4.77 & 4.25 & 4.15 & 4.46 & 4.37 & 4.65 & 4.59 & 4.45 & 4.33 & 4.47 & 4.37 & 4.51 & 4.53 & 4.49 & 4.04 & 4.05 & 4.15 & 4.27 & 4.16 & 4.11 & 4.49 & 4.28 \\
\hline Oil Consumption & & & 10.00 & 4.00 & 4.00 & 0.00 & 4.00 & 4.00 & 4.00 & 6.00 & 11.00 & 4.00 & 0.00 & 6.00 & 2.00 & 4.00 & 4.00 & 7.00 & 4.00 & 8.00 & 2.00 & 30.00 & 58.00 \\
\hline Oll Qts per 1000 & & & 2.08 & 1.66 & 1.38 & 1.12 & 1.02 & 0.96 & 0.92 & 0.98 & 1.17 & 1.13 & 1.02 & 1.04 & 0.98 & 0.97 & 0.96 & 0.98 & 0.97 & 1.02 & 1.00 & 0.92 & 1.04 \\
\hline End Odometer & 1,446 & 6,818 & 11,634 & 15,275 & 19,845 & 22,820 & 28,358 & 33,866 & 39,351 & 43,498 & 47,053 & 52,106 & 56,883 & 61,674 & 67,185 & 71,563 & 76,592 & 82,175 & 87,440 & 91,538 & 95,206 & 39,351 & 95,206 \\
\hline
\end{tabular}




\begin{tabular}{|c|c|c|c|c|c|c|c|c|c|c|c|c|c|c|c|c|c|c|c|c|c|c|c|}
\hline & Apr-97 & \begin{tabular}{|l|}
$2 y-97$ \\
\end{tabular} & In-97 & al-97 & ug-97| & Sep-97 & ct-97| & \begin{tabular}{|l|} 
Nov-97 \\
\end{tabular} & ec-97| & Jen-98 & Feb-98 & Mar-98 & Apr-98 & \begin{tabular}{|c|}
$\mathrm{May}-98$ \\
\end{tabular} & Jun-98 & Jul-98 & Aug-98 & Sep-98 & Oet-98 & \begin{tabular}{|l|} 
Nov-98 \\
\end{tabular} & Dec-98 & Total & \\
\hline tal & 018 & 524 & 661 & 315 & 630 & 489 & 8,814 & 4,855 & 4,566 & 5,627 & 4,353 & 5,113 & 3,788 & 2,281 & 4,669 & 2,897 & 5,044 & 4,557 & 3,302 & 4,447 & \begin{tabular}{l|l|}
3,936 \\
\end{tabular} & 42,972 & \\
\hline el & 2,018 & 303 & 4,155 & 3,963 & 4,882 & 5,489 & 14 & 4,855 & 4,238 & 5,627 & 3,231 & 4,725 & 3,308 & 2,281 & 4,242 & 2,555 & 4,618 & 3,972 & 2,916 & 3,657 & (2) & & \\
\hline tel I & 2633.19 & 6706.88 & 5348.18 & 5798.97 & 6271.69 & 7130.09 & 7297.53 & 6498.46 & 5676.72 & 7128.18 & 4521.66 & 6139.60 & 4216.15 & |2939.60 & 5467.72 & |3596.18 & 6237.93 & 5441.47 & 3952.69 & 4810.14 & 4280.43 & 71 & 5873 \\
\hline$\sqrt{2}$ & 45.9 & $1,900.0$ & 515.1 & 542.8 & 76.7 & 019.9 & & & & & 1, & & $1,194.4$ & 832.7 & & 8.7 & & 441.5 & 1,230 & 2.6 & 7.7 & & \\
\hline el (D2 EQQ) & 7 & (2) & & 3.7 & 9 & 29.5 & 37.9 & $1,102.3$ & 963.0 & $1,209.2$ & 767.0 & .5 & 715.2 & 498.7 & .5 & 610.0 & .058 .2 & 23.0 & 736. & 16.0 & 442.9 & 51.9 & \\
\hline G & & & & & & 2.72 & 2.81 & 64 & 2.64 & 2.79 & 2.52 & 2.72 & 2.77 & 2.74 & 4 & 2.51 & 2.61 & .58 & 2.3 & 68 & 2.5 & 2.69 & \\
\hline of $>>>$ & 4.52 & 4.66 & 4.5 & 4.03 & 4.59 & 4.54 & 4.70 & 4.40 & 4.40 & 4.65 & 4.21 & 4.54 & 4.63 & 4.57 & 4.57 & 4.19 & 4.36 & 4.30 & 3.96 & 4.48 & 4.2 & 4.5 & \\
\hline & & & 0.00 & 6.00 & 5.00 & 0.00 & 10.00 & 13.00 & 8.00 & 6.00 & 16.00 & 4.00 & 4.00 & 0.00 & 8.00 & 4.00 & 0.00 & 7.00 & 0.00 & 8.00 & 7.00 & 42.00 & 1. $13-1$ \\
\hline 100 & & & 0.00 & 0.67 & 0.75 & 0.55 & 0.81 & 1.11 & 1.19 & 1.17 & 1.41 & 1.35 & 1.33 & 1.27 & 1.31 & 1.31 & 1.22 & 1.24 & 1.18 & 1.22 & 1.24 & 1.1 & \\
\hline & & & & & & & & & 119 & 746 & 0999 & 3,212 & 2,000 & $281+>$ & 950 & & 891 & 4480 & & 9,197 & & & \\
\hline
\end{tabular}

End Odometer

LNG Yard Tractor

\begin{tabular}{|c|c|c|c|c|c|c|c|c|c|c|c|c|c|c|c|c|c|c|c|c|c|c|}
\hline Hours & $\frac{2030}{32}$ & Maty $y$ ? & & 然 & Aug $=97$ & & 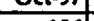 & (x) & No & int $\rightarrow 0$ & 0 & & & & & & Aug 90 & epporo & 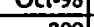 & Nov-98 & vec-98 & Total \\
\hline NG, (bs) & 32 & 305 & 313 & 194] & 166 & 157 & 186 & 156 & 201 & 223 & 162 & 170 & 185 & 223 & 260 & 273 & 299 & 315 & . & $2+0$ & 342 & 4,811 \\
\hline $\begin{array}{l}\text { (G, lbs) } \\
\text { I gal }\end{array}$ & 668.80 & 5234.32 & 6005.96 & 3599.61 & 2856.12 & 2533.57 & 2933.59| & 1996.73 & 2490.72 & 2833.33 & 1518.82 & 2132.49 & 2307.02 & 3226.43 & 3621.96 & 4618.15 & 5490.57 & 5750.94 & 4531.14 & 5154.39 & 571.51] & 6.17 \\
\hline zal & 189.5 & $1,482.8$ & $1,701.4$ & $1,019.7$ & 809.1 & 717.7 & 831.0 & 565.6 & 705.6 & 802.6 & 430.3 & 604.1 & 653.5 & 914.0 & $1,026.1$ & $1,308.3$ & $1,555.4$ & $1,629.2$ & $1,283.6$ & $1,460.2$ & $1,440.9$ & $21,130.6$ \\
\hline Fuel (D & 113.5 & 887.9 & $1,018.8$ & $\mid 0.6$ & 84.5 & 429.8 & 497.6 & 338.7 & 422.5 & 480.6 & 257.6] & 361.7 & 391.3 & 547.3 & 614.4 & 783.4 & 931.4 & 975.5 & 768.6 & 874.4 & 862.8 & $12,653.1$ \\
\hline GPH & 5.92 & 4.86 & 5.43 & 5.27 & 4.87 & 4.57 & 4.48] & 3.62 & 3.52 & 3.59 & 2.66 & 3.55 & 3.53 & 4.10 & 3.95 & 4.79 & 5.20 & 5.17 & 4.16 & 4.29 & 4.2 & 4.39 \\
\hline EGPH & 3.55 & 2.91 & 3.25 & 3.15 & 2.92 & 2.73 & 2.68 & 2.17 & 2.11 & 2.15 & 1.59 & 2.13 & 2.12 & 2.45 & 2.36 & 2.87 & 3.11 & 3.10 & 2.49 & 2.57 & 2.52 & 2.63 \\
\hline End Hours & 74.0 & 379.0 & 692.4 & 886.0 & $1,052.0$ & $1,209.2$ & $1,394.9$ & $1,551.0$ & $1,751.6$ & $1,975.0$ & $2,137.0$ & $2,307.0$ & $2,492.0$ & $2,715.0$ & $2,975.0$ & $3,248.0$ & $3,547.0$ & $3,862.1$ & $4,171.0$ & $4,511.0$ & $4,852.9$ & $4,852.9$ \\
\hline
\end{tabular}

\begin{tabular}{|c|c|c|c|c|c|c|c|c|c|c|c|c|c|c|c|c|c|c|c|c|c|c|}
\hline & Anr-077 & 10 & 0.07 & & & 7 & $=$ & Xis & 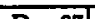 & 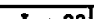 & Th & Mog & 190 & Mong & 7001 & Tog & 19 & 90 & 0 ct.-9a & (1) & (1) & \\
\hline Hours & & & & & & 13 & $\frac{c m}{307}$ & $\frac{1800-91}{344}$ & $\frac{1}{333}$ & 313 & 321 & 392 & 408 & \begin{tabular}{r|} 
May -90 \\
412
\end{tabular} & 376 & 417 & $\frac{8-0}{393}$ & 388 & 407 & $\frac{\mid>-70}{387}$ & 362 & $\frac{\text { Total }}{6.390}$ \\
\hline Fuel (LNG, lbs) & & & & 297428 & 3314,49 & 3639.59 & 3666.29 & 3837.44 & 3363.97 & 3647.47 & 2678.56 & 4455.58 & 4256.00 & 3900.87 & 3876.47 & 4928.84 & 5542.35 & 5000.97 & 4337.31 & 4710.60 & 4525.21 & $\begin{array}{r}6,390 \\
72656.29\end{array}$ \\
\hline LNG std gal & & & & 842.6 & 938.9 & 1031.0 & 1038.6 & $1,087.1$ & 953.0 & $1,033.3$ & 758.8 & $1,262.2$ & $1,205.7$ & $1,105.1$ & $1,098.2$ & $1,396.3$ & $1,570.1$ & $1,416.7$ & $1,228.7$ & $1,334.4$ & 1.376 .3 & $20,676.9$ \\
\hline Fuel (D2 EQ) & & & & 504.5 & 562.2 & 617.4 & 621.9 & 651.0 & 570.6 & 618.7 & 454.4 & 755.8 & 722.0 & 661.7 & 657.6 & 836.1 & 940.2 & 848.3 & 735.7 & 799.1 & 824.1 & $12,381.4$ \\
\hline GPH & & & & 3.58 & 3.25 & 3.38 & 3.39 & 3.16 & 2.86 & 3.30 & 2.36 & 3.22 & 2.96 & 2.68 & 2.92 & 3.35 & 4.00 & 3.65 & 3.02 & 3.45 & 3.80 & 3.24 \\
\hline EGPH & & & & 2.14 & 1.95 & 2.02 & 2.03 & 1.89 & 1.71 & 1.98 & 1.42 & 1.93 & 1.77 & 1.61 & 1.75 & 2.01 & 2.39 & 2.19 & 1.81 & 2.06 & 2.28 & 1.94 \\
\hline End Hours & & & & 239.0 & 528.0 & 833.2 & $1,139.9$ & $1,484.0$ & $1,817.2$ & $2,130.0$ & $2,451.0$ & $2,843.0$ & $3,251.0$ & $3,663.0$ & $4,039.0$ & $4,456.0$ & $4,849.0$ & $5,237.0$ & $5,644.0$ & $6,031.0$ & $6,393.0$ & $6,393.0$ \\
\hline
\end{tabular}

End Hours

GPH - gallons per hour

EGPH - energy equivalent diesel \#2 gallons per hour

MPG - miles per gallon

MPEG - miles per energy equivalent diesel \#2 gallon 

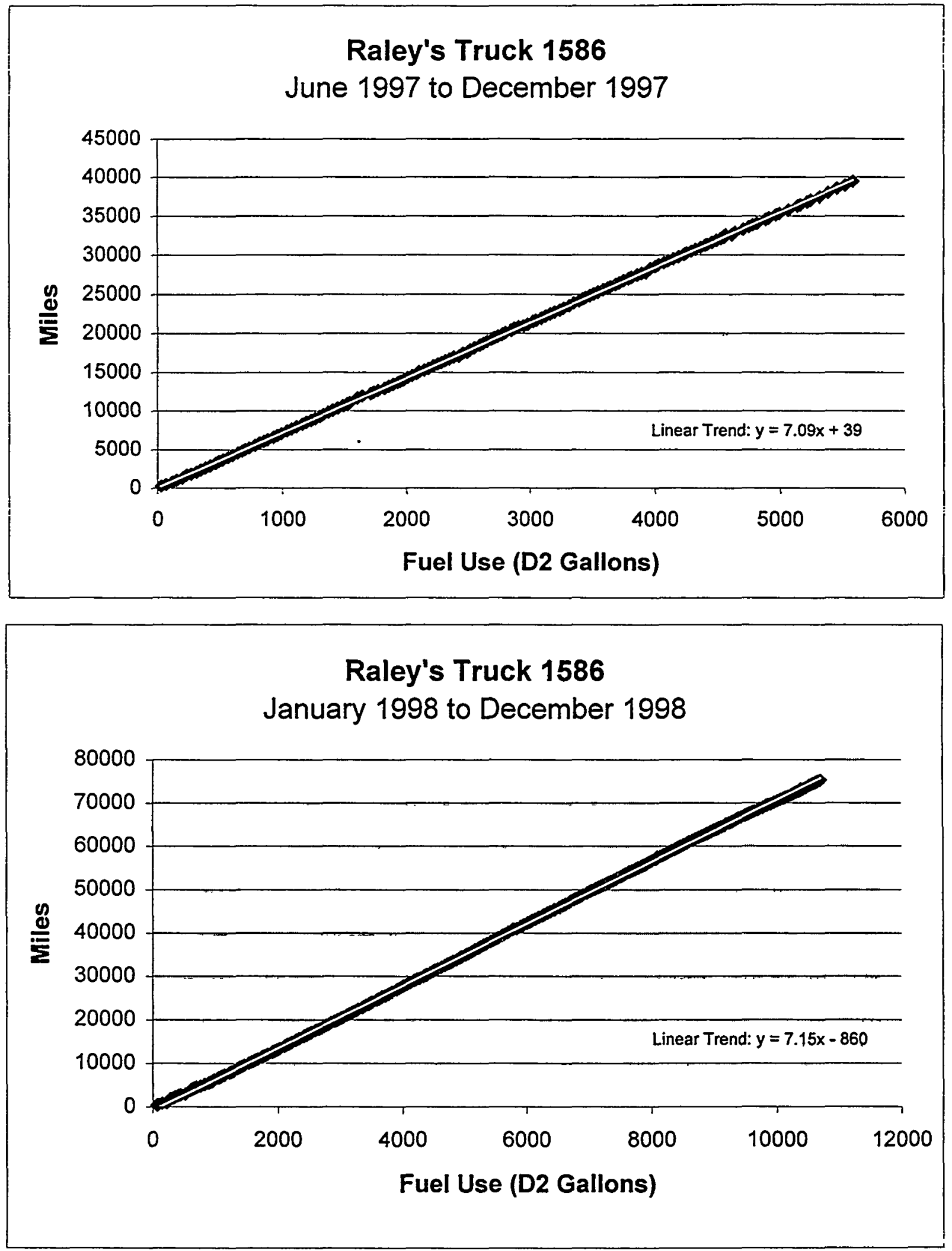

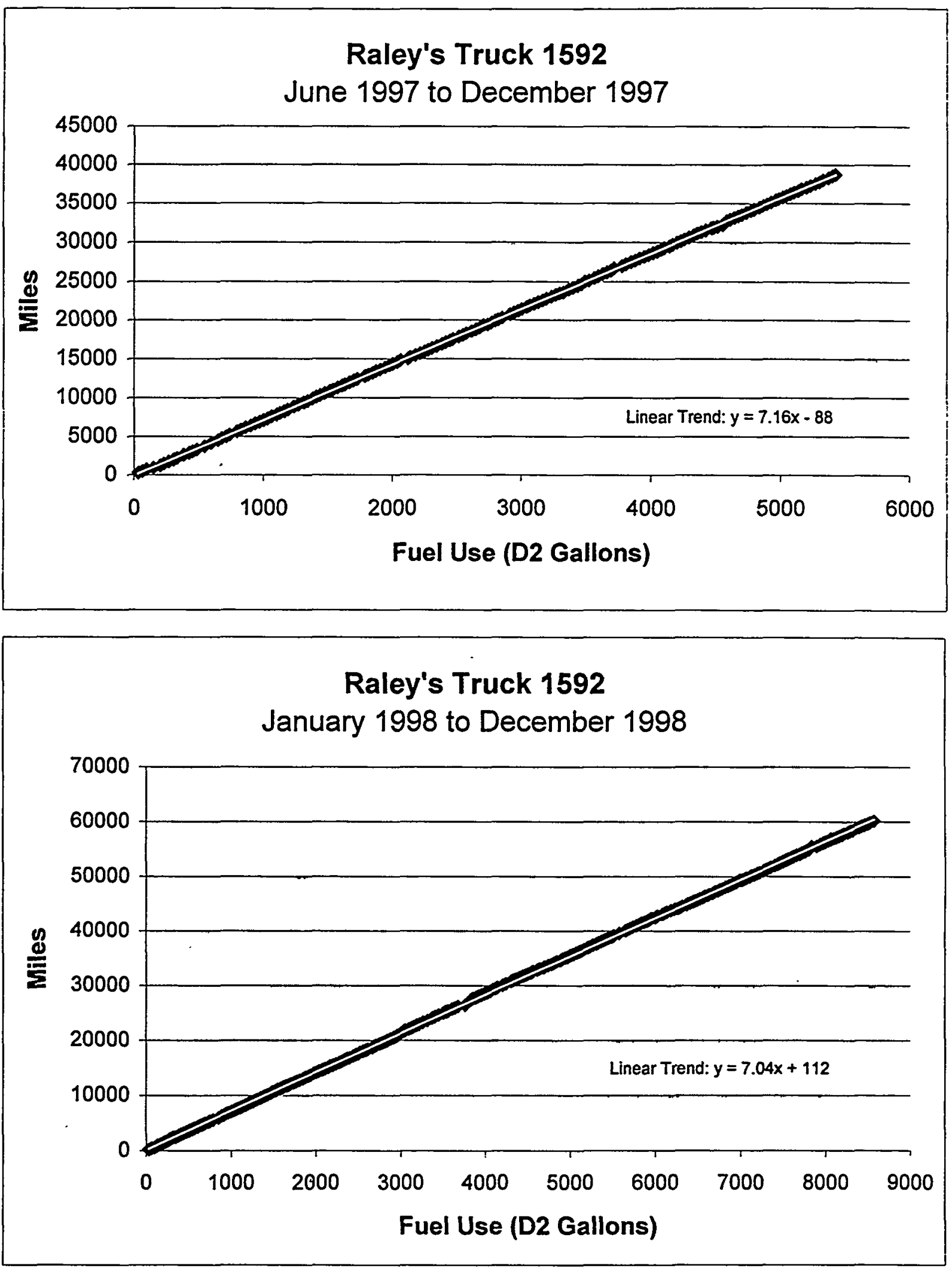

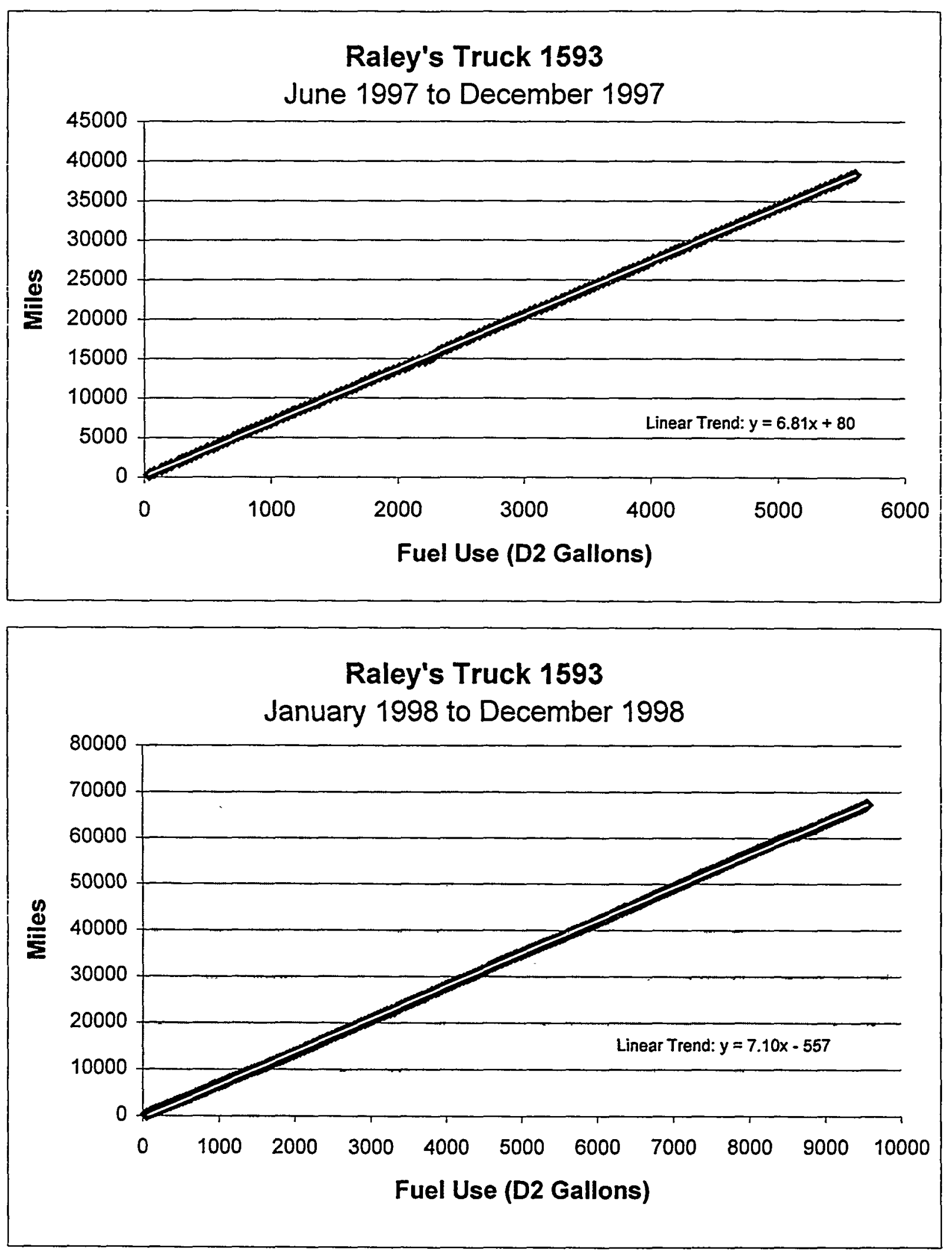

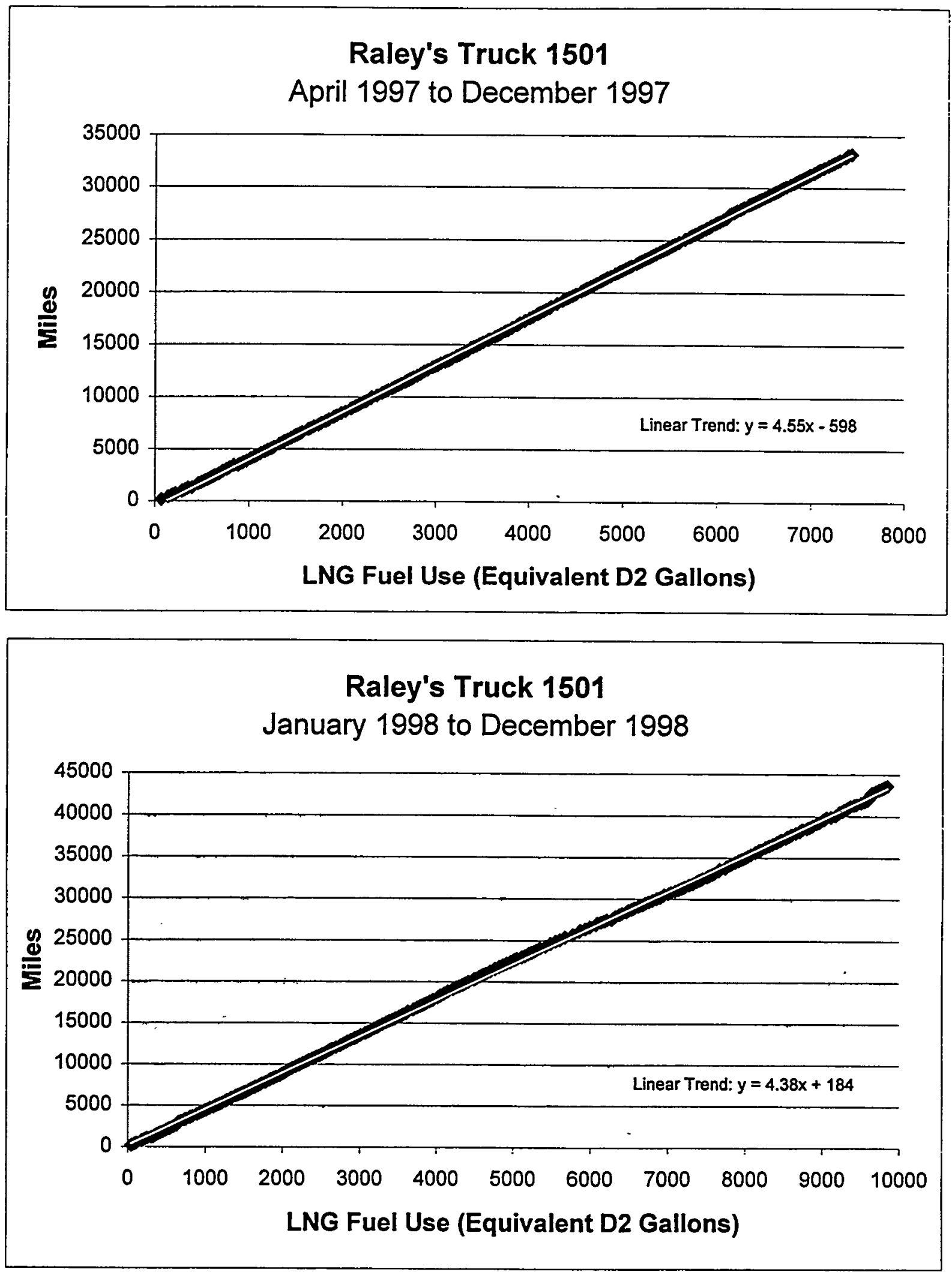

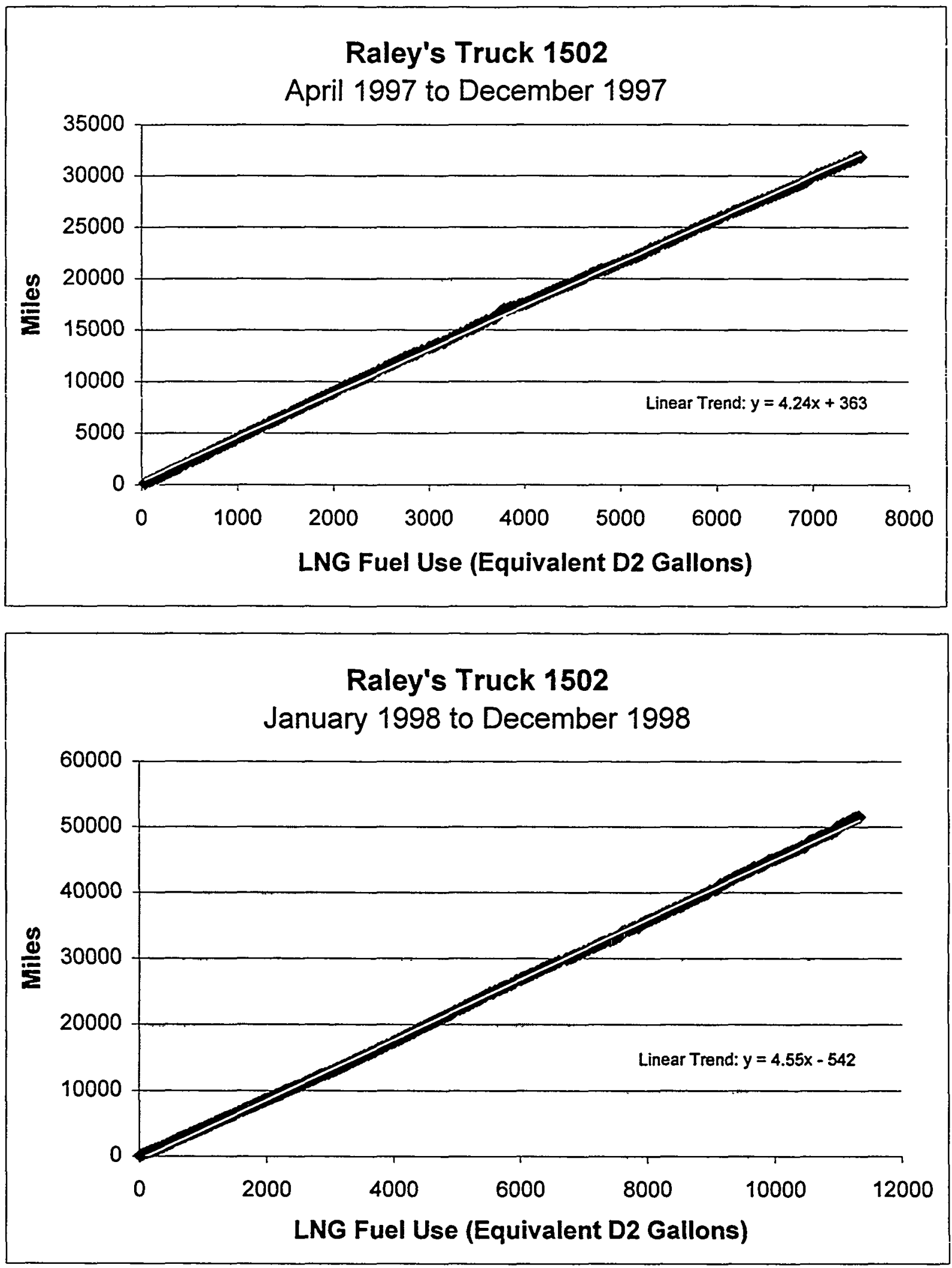

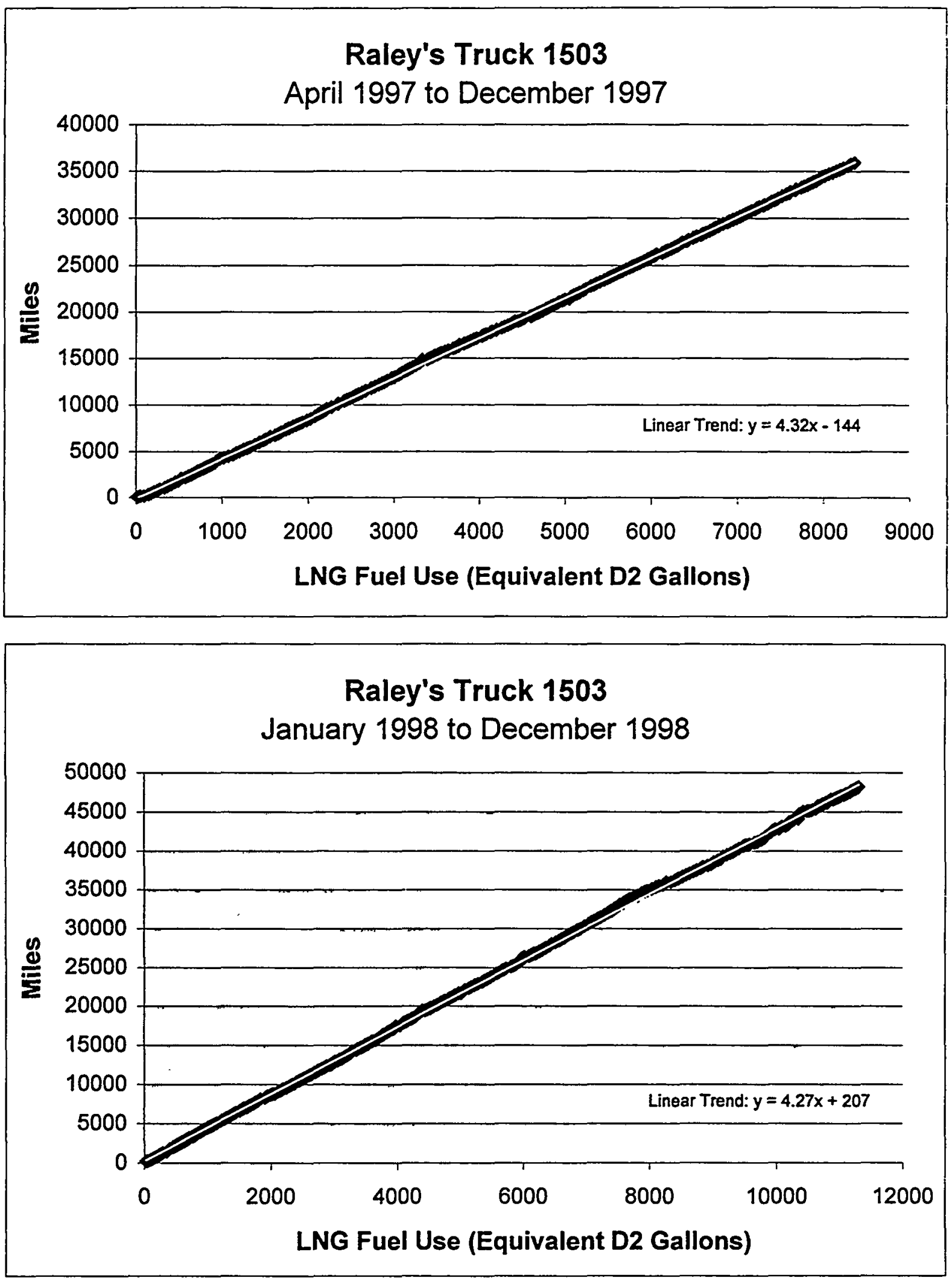

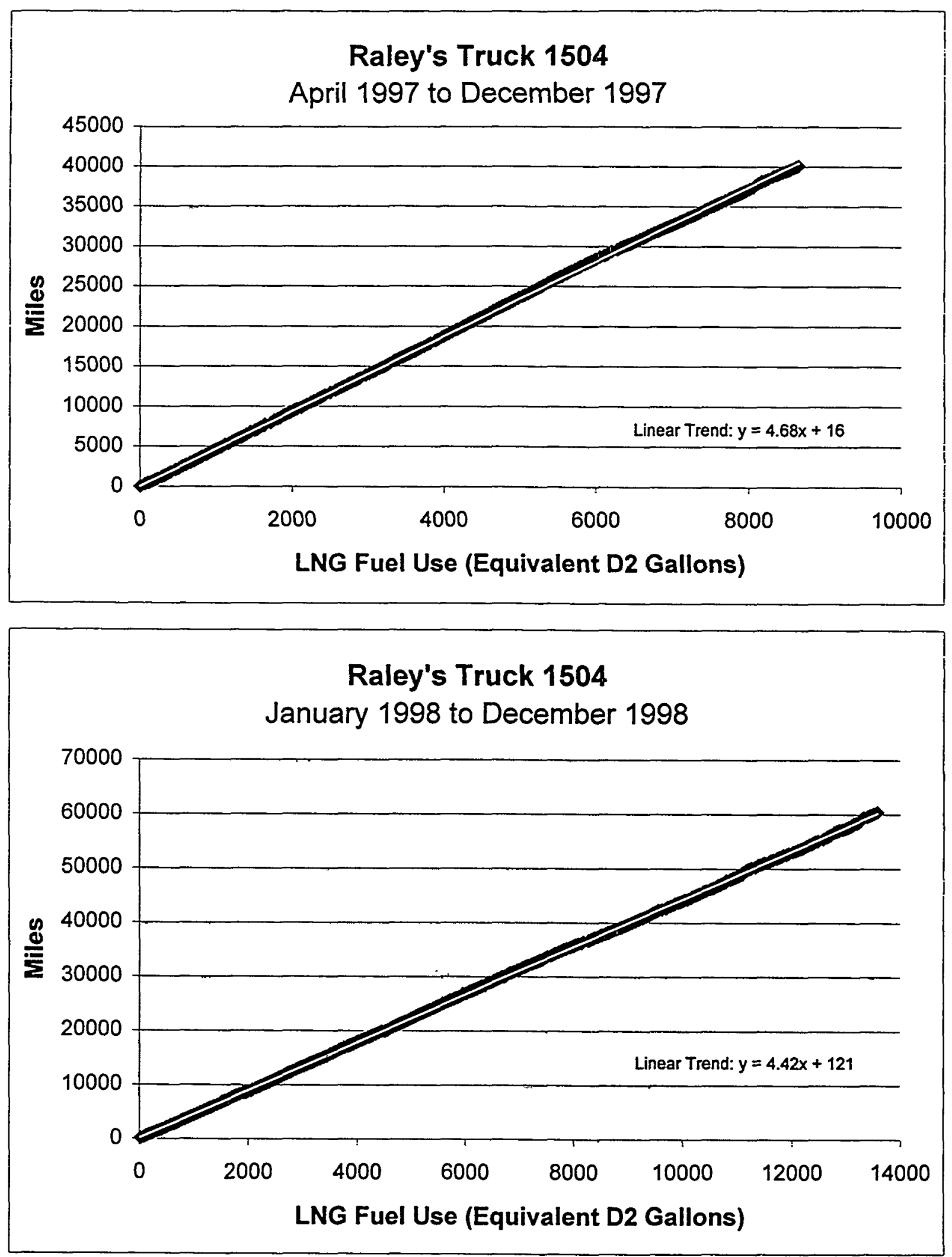

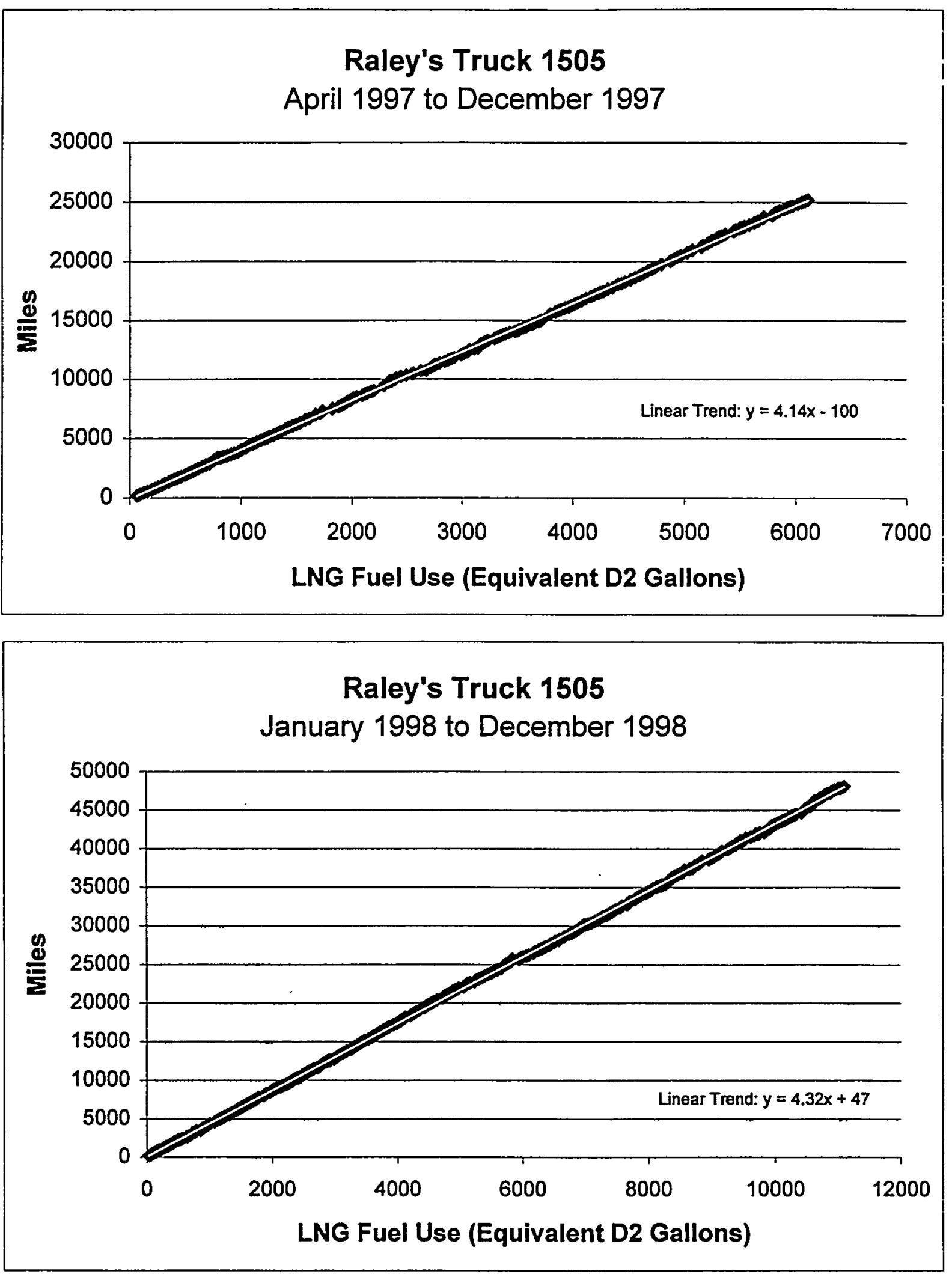

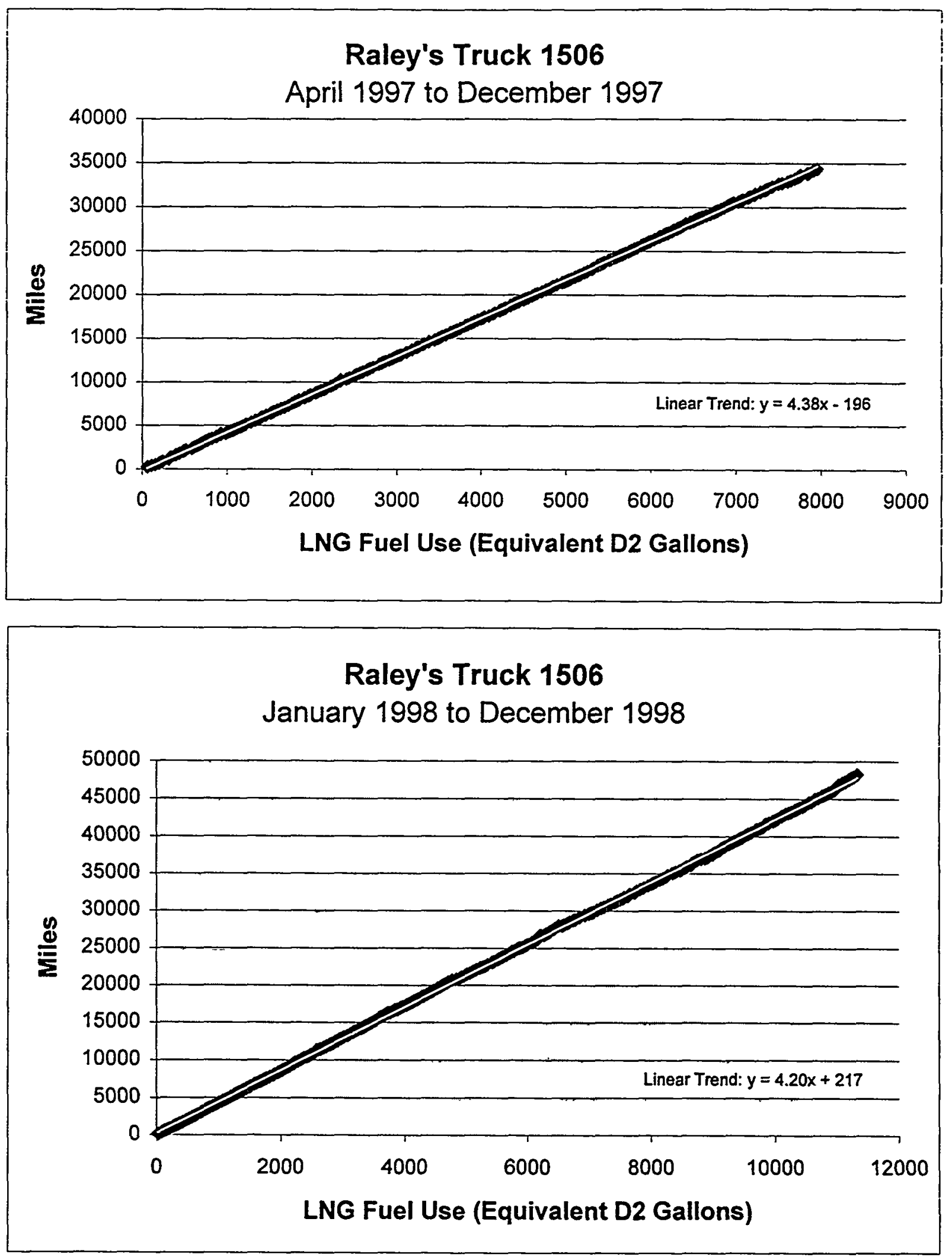

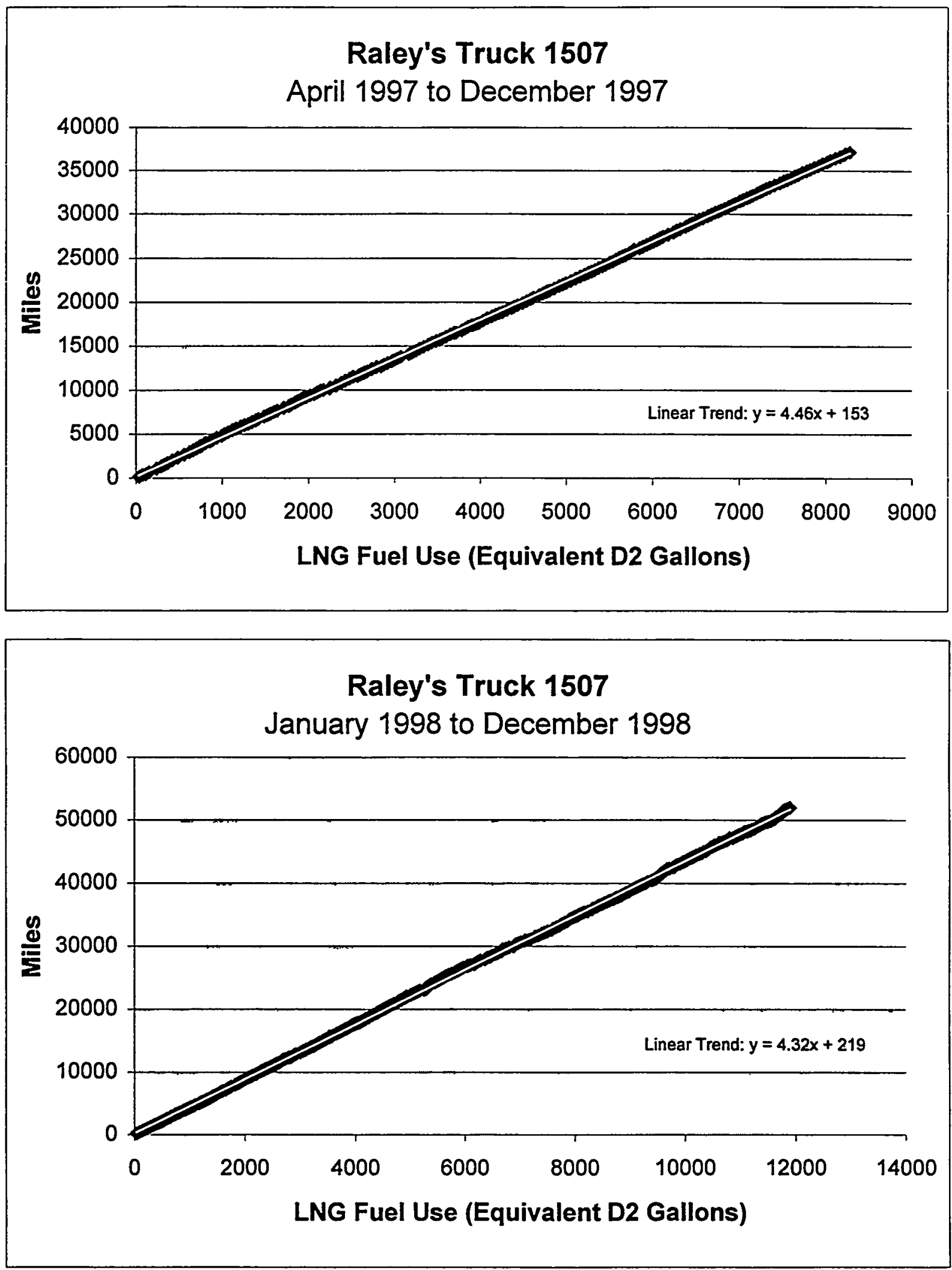

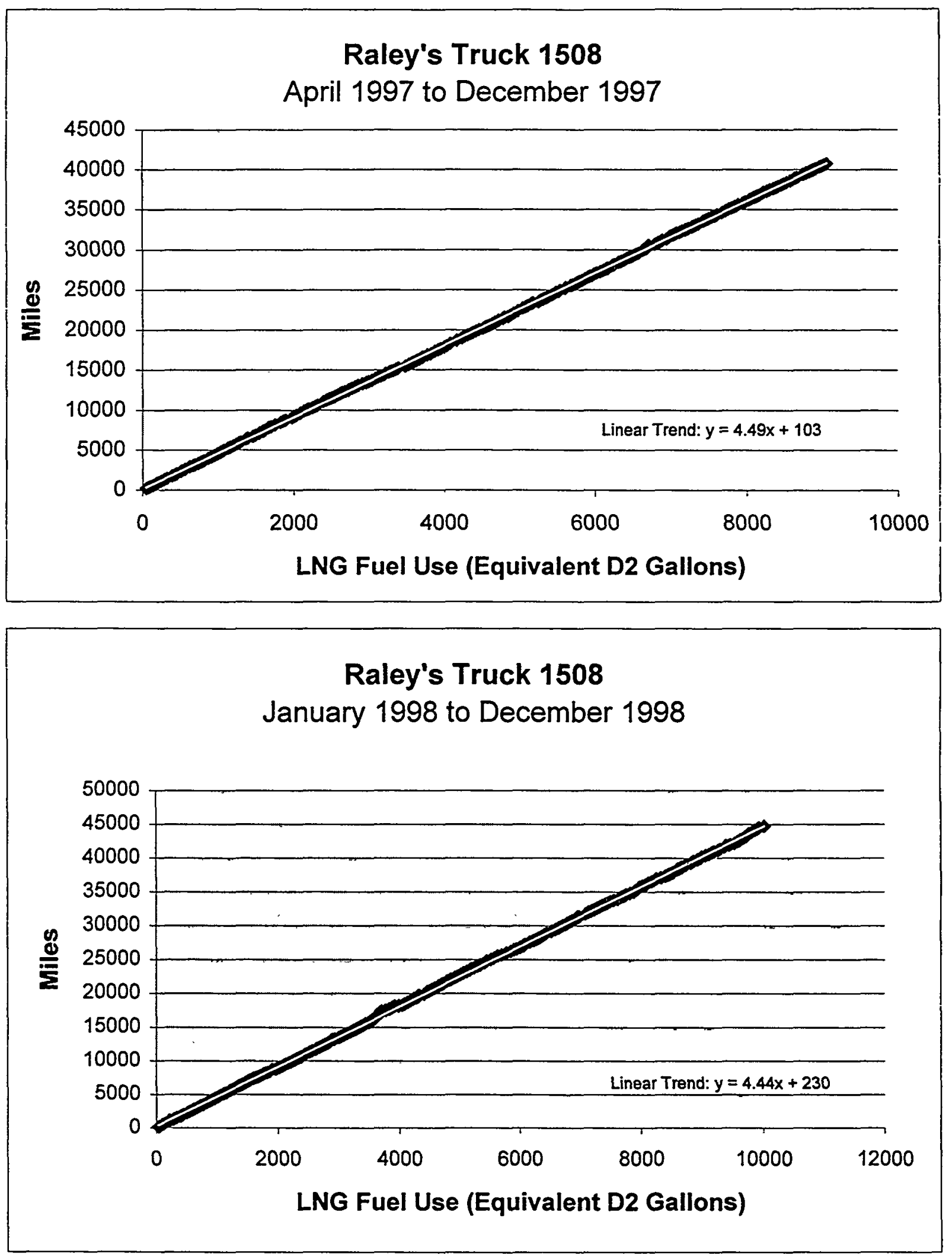


\section{Appendix F}

\section{LNG and Diesel Fuel Costs}


Raley's Fuel Shipments from JBK

\begin{tabular}{|c|c|c|c|c|c|c|}
\hline Date & Amount & Cost/Gal & Fuel Cost & Other Cost & Total Cost & Cost/Gal \\
\hline $4 / 8 / 97$ & 8614 & 0.55 & 4737.70 & 465.00 & 5202.70 & 0.604 \\
\hline $4 / 28 / 97$ & 5440 & 0.55 & 2992.00 & 0.00 & 2992.00 & 0.550 \\
\hline $5 / 8 / 97$ & 5606 & 0.55 & 3083.30 & 0.00 & 3083.30 & 0.550 \\
\hline $5 / 19 / 97$ & 4607 & 0.55 & 2533.85 & 0.00 & 2533.85 & 0.550 \\
\hline $5 / 27 / 97$ & 5080 & 0.55 & 2794.00 & 0.00 & 2794.00 & 0.550 \\
\hline $6 / 5 / 97$ & 5524 & 0.55 & 3038.20 & 0.00 & 3038.20 & 0.550 \\
\hline $6 / 16 / 97$ & 5012 & 0.55 & 2756.60 & 0.00 & 2756.60 & 0.550 \\
\hline $6 / 25 / 97$ & 4985 & 0.55 & 2741.75 & 120.00 & 2861.75 & 0.574 \\
\hline $7 / 6 / 97$ & 5112 & 0.55 & 2811.60 & 0.00 & 2811.60 & 0.550 \\
\hline $7 / 16 / 97$ & 4265 & 0.55 & 2345.75 & 0.00 & 2345.75 & 0.550 \\
\hline $7 / 28 / 97$ & 4518 & 0.55 & 2484.90 & 0.00 & 2484.90 & 0.550 \\
\hline $8 / 8 / 97$ & 5001 & 0.55 & 2750.55 & 0.00 & 2750.55 & 0.550 \\
\hline $8 / 20 / 97$ & 4968 & 0.55 & 2732.40 & 0.00 & 2732.40 & 0.550 \\
\hline $8 / 29 / 97$ & 5035 & 0.55 & 2769.25 & 0.00 & 2769.25 & 0.550 \\
\hline $9 / 5 / 97$ & 3500 & 0.55 & 1925.00 & 0.00 & 1925.00 & 0.550 \\
\hline $9 / 15 / 97$ & 4963 & 0.55 & 2729.65 & 0.00 & 2729.65 & 0.550 \\
\hline $9 / 25 / 97$ & 5195 & 0.55 & 2857.25 & 0.00 & 2857.25 & 0.550 \\
\hline $10 / 4 / 97$ & 4990 & 0.55 & 2744.50 & 0.00 & 2744.50 & 0.550 \\
\hline $10 / 12 / 97$ & 5000 & 0.55 & 2750.00 & 0.00 & 2750.00 & 0.550 \\
\hline $10 / 24 / 97$ & 5479 & 0.55 & 3013.45 & 0.00 & 3013.45 & 0.550 \\
\hline $11 / 3 / 97$ & 5480 & 0.55 & 3014.00 & 0.00 & 3014.00 & 0.550 \\
\hline $11 / 13 / 97$ & 5447 & 0.55 & 2995.85 & 0.00 & 2995.85 & 0.550 \\
\hline $11 / 22 / 97$ & 5502 & 0.55 & 3026.10 & 0.00 & 3026.10 & 0.550 \\
\hline $12 / 4 / 97$ & 4857 & 0.55 & 2671.35 & 0.00 & 2671.35 & 0.550 \\
\hline $12 / 16 / 97$ & 4657 & 0.55 & 2561.35 & 0.00 & 2561.35 & 0.550 \\
\hline $12 / 25 / 97$ & 4735 & 0.55 & 2604.25 & 0.00 & 2604.25 & 0.550 \\
\hline $1 / 5 / 98$ & 5046 & 0.55 & 2775.30 & 0.00 & 2775.30 & 0.550 \\
\hline $1 / 19 / 98$ & 4790 & 0.55 & 2634.50 & 0.00 & 2634.50 & 0.550 \\
\hline $1 / 28 / 98$ & 4954 & 0.55 & 2724.70 & 0.00 & 2724.70 & 0.550 \\
\hline $2 / 8 / 98$ & 4751 & 0.55 & 2613.05 & 0.00 & 2613.05 & 0.550 \\
\hline $2 / 18 / 98$ & 4904 & 0.55 & 2697.20 & 0.00 & 2697.20 & 0.550 \\
\hline $3 / 1 / 98$ & 4643 & 0.55 & 2553.65 & 0.00 & 2553.65 & 0.550 \\
\hline $3 / 8 / 98$ & 4351 & 0.55 & 2393.05 & 0.00 & 2393.05 & 0.550 \\
\hline $3 / 16 / 98$ & 4565 & 0.55 & 2510.75 & 0.00 & 2510.75 & 0.550 \\
\hline $3 / 27 / 98$ & 4612 & 0.55 & 2536.60 & 0.00 & 2536.60 & 0.550 \\
\hline $4 / 5 / 98$ & 4946 & 0.55 & 2720.30 & 0.00 & 2720.30 & 0.550 \\
\hline $4 / 12 / 98$ & 4612 & 0.55 & 2536.60 & 0.00 & 2536.60 & 0.550 \\
\hline $4 / 19 / 98$ & 4340 & 0.575 & 2495.50 & 0.00 & 2495.50 & 0.575 \\
\hline $4 / 29 / 98$ & 4587 & 0.575 & 2637.53 & 0.00 & 2637.53 & 0.575 \\
\hline $5 / 7 / 98$ & 4679 & 0.575 & 2690.43 & 0.00 & 2690.43 & 0.575 \\
\hline $5 / 17 / 98$ & 4729 & 0.575 & 2719.18 & 0.00 & 2719.18 & 0.575 \\
\hline $5 / 26 / 98$ & 4599 & 0.575 & 2644.43 & 0.00 & 2644.43 & 0.575 \\
\hline $6 / 6 / 98$ & 4624 & 0.575 & 2658.80 & 0.00 & 2658.80 & 0.575 \\
\hline $6 / 12 / 98$ & 4451 & 0.575 & 2559.33 & 0.00 & 2559.33 & 0.575 \\
\hline $6 / 28 / 98$ & 4801 & 0.575 & 2760.58 & 0.00 & 2760.58 & 0.575 \\
\hline $7 / 5 / 98$ & 5154 & 0.575 & 2963.55 & 300.00 & 3263.55 & 0.633 \\
\hline $7 / 18 / 98$ & 9961 & 0.475 & 4731.48 & 1180.00 & 5911.48 & 0.593 \\
\hline $7 / 24 / 98$ & 4104 & 0.575 & 2359.80 & 0.00 & 2359.80 & 0.575 \\
\hline $7 / 31 / 98$ & 4871 & 0.575 & 2800.83 & 0.00 & 2800.83 & 0.575 \\
\hline $8 / 8 / 98$ & 4557 & 0.575 & 2620.28 & 0.00 & 2620.28 & 0.575 \\
\hline $8 / 19 / 98$ & 4421 & 0.575 & 2542.08 & 0.00 & 2542.08 & 0.575 \\
\hline $8 / 27 / 98$ & 4762 & 0.575 & 2738.15 & 0.00 & 2738.15 & 0.575 \\
\hline $9 / 12 / 98$ & 5049 & 0.575 & 2903.18 & 0.00 & 2903.18 & 0.575 \\
\hline $10 / 1 / 98$ & 4843 & 0.575 & 2784.73 & 0.00 & 2784.73 & 0.575 \\
\hline $10 / 13 / 98$ & 4407 & 0.575 & 2534.03 & 0.00 & 2534.03 & 0.575 \\
\hline $10 / 20 / 98$ & 3871 & 0.575 & 2225.83 & 0.00 & 2225.83 & 0.575 \\
\hline $10 / 28 / 98$ & 4585 & 0.575 & 2636.38 & 0.00 & 2636.38 & 0.575 \\
\hline $11 / 9 / 98$ & 4479 & 0.575 & 2575.43 & 0.00 & 2575.43 & 0.575 \\
\hline $11 / 17 / 98$ & 4040 & 0.575 & 2323.00 & 0.00 & 2323.00 & 0.575 \\
\hline $11 / 24 / 98$ & 5421 & 0.575 & 3117.08 & 0.00 & 3117.08 & 0.575 \\
\hline $12 / 4 / 98$ & 4846 & 0.575 & 2786.45 & 0.00 & 2786.45 & 0.575 \\
\hline $12 / 10 / 98$ & 9469 & 0.475 & 4997.78 & 0.00 & 4497.78 & 0.475 \\
\hline $12 / 15 / 98$ & 4276 & 0.575 & 2458.70 & 0.00 & 2458.70 & 0.575 \\
\hline $12 / 29 / 98$ & 4412 & 0.575 & 2536.90 & 0.00 & 2536.90 & 0.575 \\
\hline $1 / 11 / 99$ & 6191 & 0.575 & 3559.83 & 0.00 & 3559.83 & 0.575 \\
\hline $1 / 26 / 99$ & 9949 & 0.475 & 4725.78 & 0.00 & 4725.78 & 0.475 \\
\hline Total & 336224 & & 185747.25 & 2065.00 & 187812.25 & 0.559 \\
\hline
\end{tabular}




\begin{tabular}{|c|c|c|c|c|c|c|c|}
\hline Truck & Date & Fuel & Amount & Odometer & Cost & Adj. Cost & Monthly Avg \\
\hline 1592 & $7 / 18 / 97$ & Diesel & 47.62 & 129311 & 52.860 & 49.5266 & \\
\hline 1593 & $7 / 18 / 97$ & Diesel & 40.01 & 162980 & 44.410 & 41.6093 & \\
\hline 1586 & $7 / 19 / 97$ & Diesel & 37.06 & 156852 & 41.140 & 38.5458 & \\
\hline 1593 & $7 / 19 / 97$ & Diesel & 19.00 & 163077 & 21.090 & 19.76 & \\
\hline 1586 & $7 / 21 / 97$ & Diesel & 36.63 & 157133 & 40.660 & 38.0959 & \\
\hline 1592 & $7 / 21 / 97$ & Diesel & 32.59 & 129547 & 36.170 & 33.8887 & \\
\hline 1586 & $7 / 22 / 97$ & Diesel & 48.55 & 157454 & 53.890 & 50.4915 & \\
\hline 1592 & $7 / 22 / 97$ & Diesel & 44.24 & 129827 & 49.110 & 46.0132 & \\
\hline 1593 & $7 / 22 / 97$ & Diesel & 43.00 & 163367 & 47.730 & 44.72 & \\
\hline 1586 & $7 / 23 / 97$ & Diesel & 32.49 & 157704 & 36.060 & 33.7857 & \\
\hline 1592 & $7 / 23 / 97$ & Diesel & 27.81 & 130037 & 30.870 & 28.9233 & \\
\hline 1593 & $7 / 23 / 97$ & Diesel & 41.78 & 163826 & 46.380 & 43.4554 & \\
\hline 1586 & $7 / 24 / 97$ & Diesel & 40.42 & 157967 & 43.650 & 40.8206 & \\
\hline 1592 & $7 / 24 / 97$ & Diesel & 39.98 & 130334 & 43.180 & 40.3814 & \\
\hline 1593 & $7 / 24 / 97$ & Diesel & 36.37 & 164089 & 39.280 & 36.7341 & \\
\hline 1586 & $7 / 25 / 97$ & Diesel & 32.01 & 158195 & 34.570 & 32.3293 & \\
\hline 1592 & $7 / 25 / 97$ & Diesel & 26.71 & 130547 & 28.850 & 26.9803 & \\
\hline 1593 & $7 / 25 / 97$ & Diesel & 38.99 & 164362 & 42.110 & 39.3807 & \\
\hline 1586 & $7 / 25 / 97$ & Diesel & 36.81 & 158479 & 39.750 & 37.1733 & \\
\hline 1586 & $7 / 26 / 97$ & Diesel & 29.12 & 158703 & 31.450 & 29.4116 & \\
\hline 1592 & $7 / 26 / 97$ & Diesel & 30.07 & 130759 & 32.480 & 30.3751 & \\
\hline 1586 & $7 / 28 / 97$ & Diesel & 38.86 & 158996 & 41.970 & 39.2498 & \\
\hline 1592 & $7 / 28 / 97$ & Diesel & 35.40 & 131015 & 38.230 & 35.752 & \\
\hline 1593 & $7 / 28 / 97$ & Diesel & 60.02 & 165044 & 64.820 & 60.6186 & \\
\hline 1586 & $7 / 29 / 97$ & Diesel & 36.56 & 159250 & 42.780 & 40.2208 & \\
\hline 1592 & $7 / 29 / 97$ & Diesel & 36.28 & 131261 & 42.450 & 39.9104 & \\
\hline 1593 & $7 / 29 / 97$ & Diesel & 39.52 & 165325 & 46.240 & 43.4736 & \\
\hline 1586 & $7 / 30 / 97$ & Diesel & 40.65 & 159531 & 47.560 & 44.7145 & \\
\hline 1592 & $7 / 30 / 97$ & Diesel & 28.48 & 131473 & 33.320 & 31.3264 & \\
\hline 1593 & $7 / 30 / 97$ & Diesel & 32.07 & 165404 & 37.520 & 35.2751 & \\
\hline 1586 & $7 / 31 / 97$ & Diesel & 42.26 & 159816 & 49.440 & 46.4818 & \\
\hline 1592 & $7 / 31 / 97$ & Diesel & 35.61 & 131723 & 41.660 & 39.1673 & \\
\hline 1593 & $7 / 31 / 97$ & Diesel & 38.55 & 165776 & 45.100 & 42.4015 & 1.05 \\
\hline 1586 & $8 / 1 / 97$ & Diesel & 36.02 & 160077 & 42.140 & 39.6186 & \\
\hline 1593 & $8 / 1 / 97$ & Diesel & 47.87 & 166108 & 56.010 & 52.6591 & \\
\hline 1592 & $8 / 1 / 97$ & Diesel & 35.61 & 131971 & 41.660 & 39.1673 & \\
\hline 1592 & $8 / 2 / 97$ & Diesel & 20.03 & 132100 & 23.440 & 22.0379 & \\
\hline 1593 & $8 / 2 / 97$ & Diesel & 51.05 & 166412 & 59.730 & 56.1565 & \\
\hline 1586 & $8 / 2 / 97$ & Diesel & 18.66 & 160250 & 21.830 & 20.5238 & \\
\hline 1586 & $8 / 4 / 97$ & Diesel & 63.32 & 160876 & 79.150 & 74.7176 & \\
\hline 1592 & $8 / 4 / 97$ & Diesel & 36.86 & 132393 & 46.080 & 43.4998 & \\
\hline 1593 & $8 / 4 / 97$ & Diesel & 35.80 & 166661 & 44.750 & 42.244 & \\
\hline 1586 & $8 / 5 / 97$ & Diesel & 38.25 & 161146 & 47.810 & 45.1325 & \\
\hline 1592 & $8 / 5 / 97$ & Diesel & 30.39 & 132637 & 37.990 & 35.8627 & \\
\hline 1593 & $8 / 5 / 97$ & Diesel & 41.35 & 166947 & 51.690 & 48.7955 & \\
\hline 1592 & $8 / 6 / 97$ & Diesel & 30.40 & 132875 & 38.000 & 35.872 & \\
\hline 1586 & $8 / 7 / 97$ & Diesel & 68.05 & 161442 & 85.060 & 80.2965 & \\
\hline 1593 & $8 / 7 / 97$ & Diesel & 41.37 & 167220 & 51.710 & 48.8141 & \\
\hline 1592 & $8 / 8 / 97$ & Diesel & 58.79 & 133219 & 74.660 & 70.5447 & \\
\hline 1586 & $8 / 8 / 97$ & Diesel & 22.75 & 161615 & 28.890 & 27.2975 & \\
\hline 1593 & $8 / 8 / 97$ & Diesel & 35.19 & 167430 & 44.690 & 42.2267 & \\
\hline 1586 & $8 / 9 / 97$ & Diesel & 34.68 & 161850 & 44.040 & 41.6124 & \\
\hline 1592 & $8 / 9 / 97$ & Diesel & 25.60 & 133411 & 32.510 & 30.718 & \\
\hline 1593 & $8 / 9 / 97$ & Diesel & 36.95 & 167745 & 46.930 & 44.3435 & \\
\hline
\end{tabular}




\begin{tabular}{|c|c|c|c|c|c|c|c|}
\hline Truck & Date & Fuel & Amount & Odometer & Cost & Adj. Cost & Monthly Avg \\
\hline 1586 & $8 / 11 / 97$ & Diesel & 53.98 & 162132 & 68.550 & 64.7714 & \\
\hline 1592 & $8 / 11 / 97$ & Diesel & 34.00 & 133639 & 43.180 & 40.8 & \\
\hline 1593 & $8 / 11 / 97$ & Diesel & 33.46 & 167993 & 42.490 & 40.1478 & \\
\hline 1586 & $8 / 12 / 97$ & Diesel & 33.10 & 162374 & 42.040 & 39.723 & \\
\hline 1592 & $8 / 12 / 97$ & Diesel & 25.81 & 133854 & 32.780 & 30.9733 & \\
\hline 1593 & $8 / 12 / 97$ & Diesel & 44.58 & 168284 & 56.620 & 53.4994 & \\
\hline 1586 & $8 / 13 / 97$ & Diesel & 36.27 & 162633 & 46.060 & 43.5211 & \\
\hline 1592 & $8 / 13 / 97$ & Diesel & 32.91 & 134068 & 41.800 & 39.4963 & \\
\hline 1593 & $8 / 13 / 97$ & Diesel & 29.84 & 168506 & 37.900 & 35.8112 & \\
\hline 1586 & $8 / 14 / 97$ & Diesel & 36.34 & 162898 & 46.150 & 43.6062 & \\
\hline 1592 & $8 / 14 / 97$ & Diesel & 39.31 & 134350 & 49.920 & 47.1683 & \\
\hline 1593 & $8 / 14 / 97$ & Diesel & 40.06 & 168763 & 50.880 & 48.0758 & \\
\hline 1586 & $8 / 15 / 97$ & Diesel & 24.44 & 163070 & 31.280 & 29.5692 & \\
\hline 1592 & $8 / 15 / 97$ & Diesel & 32.35 & 134612 & 41.410 & 39.1455 & \\
\hline 1593 & $8 / 15 / 97$ & Diesel & 47.62 & 169025 & 60.950 & 57.6166 & \\
\hline 1586 & $8 / 16 / 97$ & Diesel & 26.93 & 163255 & 34.470 & 32.5849 & \\
\hline 1592 & $8 / 16 / 97$ & Diesel & 24.98 & 134815 & 31.970 & 30.2214 & \\
\hline 1593 & $8 / 16 / 97$ & Diesel & 24.00 & 169187 & 30.720 & 29.04 & \\
\hline 1586 & $8 / 18 / 97$ & Diesel & 27.55 & 163473 & 35.260 & 33.3315 & \\
\hline 1592 & $8 / 18 / 97$ & Diesel & 33.97 & 135048 & 43.480 & 41.1021 & \\
\hline 1593 & $8 / 18 / 97$ & Diesel & 37.98 & 169426 & 48.610 & 45.9514 & \\
\hline 1586 & $8 / 19 / 97$ & Diesel & 37.45 & 163750 & 48.310 & 45.6885 & \\
\hline 1592 & $8 / 19 / 97$ & Diesel & 25.83 & 135253 & 33.320 & 31.5119 & \\
\hline 1593 & $8 / 19 / 97$ & Diesel & 46.44 & 169698 & 59.910 & 56.6592 & \\
\hline 1586 & $8 / 20 / 97$ & Diesel & 36.05 & 164000 & 46.600 & 44.0765 & \\
\hline 1592 & $8 / 20 / 97$ & Diesel & 60.19 & 135463 & 77.650 & 73.4367 & \\
\hline 1593 & $8 / 20 / 97$ & Diesel & 45.99 & 169894 & 59.330 & 56.1107 & \\
\hline 1586 & $8 / 21 / 97$ & Diesel & 18.82 & 252 & 24.280 & 22.9626 & \\
\hline 1592 & $8 / 21 / 97$ & Diesel & 15.96 & 135673 & 20.590 & 19.4728 & \\
\hline 1593 & $8 / 21 / 97$ & Diesel & 33.17 & 170148 & 42.790 & 40.4681 & \\
\hline 1586 & $8 / 22 / 97$ & Diesel & 40.60 & 475 & 52.370 & 49.528 & \\
\hline 1593 & $8 / 22 / 97$ & Diesel & 28.41 & 170409 & 36.650 & 34.6613 & \\
\hline 1592 & $8 / 23 / 97$ & Diesel & 38.98 & 135883 & 50.280 & 47.5514 & \\
\hline 1593 & $8 / 23 / 97$ & Diesel & 47.18 & 170667 & 60.860 & 57.5574 & \\
\hline 1586 & $8 / 25 / 97$ & Diesel & 37.24 & 701 & 48.040 & 45.4332 & \\
\hline 1592 & $8 / 25 / 97$ & Diesel & 38.45 & 136134 & 49.600 & 46.9085 & \\
\hline 1593 & $8 / 25 / 97$ & Diesel & 49.88 & 170799 & 64.340 & 60.8484 & \\
\hline 1586 & $8 / 26 / 97$ & Diesel & 37.76 & 996 & 48.710 & 46.0668 & \\
\hline 1593 & $8 / 26 / 97$ & Diesel & 37.99 & 171064 & 49.010 & 46.3507 & \\
\hline 1586 & $8 / 27 / 97$ & Diesel & 36.74 & 1265 & 47.390 & 44.8182 & \\
\hline 1592 & $8 / 27 / 97$ & Diesel & 35.68 & 136364 & 46.030 & 43.5324 & \\
\hline 1593 & $8 / 27 / 97$ & Diesel & 23.99 & 184 & 30.950 & 29.2707 & \\
\hline 1586 & $8 / 28 / 97$ & Diesel & 29.20 & 1454 & 37.380 & 35.336 & \\
\hline 1592 & $8 / 28 / 97$ & Diesel & 27.38 & 136571 & 35.050 & 33.1334 & \\
\hline 1592 & $8 / 29 / 97$ & Diesel & 30.30 & 136790 & 38.780 & 36.659 & \\
\hline 1593 & $8 / 29 / 97$ & Diesel & 40.57 & 730 & 51.930 & 49.0901 & \\
\hline 1586 & $8 / 29 / 97$ & Diesel & 28.89 & 1665 & 36.980 & 34.9577 & \\
\hline 1586 & $8 / 30 / 97$ & Diesel & 35.97 & 1905 & 46.040 & 43.5221 & \\
\hline 1592 & $8 / 30 / 97$ & Diesel & 37.23 & 137065 & 47.650 & 45.0439 & \\
\hline 1593 & $8 / 30 / 97$ & Diesel & 28.75 & 918 & 36.800 & 34.7875 & 1.20 \\
\hline 1586 & $9 / 1 / 97$ & Diesel & 35.46 & 2164 & 45.390 & 42.9078 & \\
\hline 1592 & $9 / 1 / 97$ & Diesel & 38.30 & 137351 & 49.020 & 46.339 & \\
\hline 1593 & $9 / 1 / 97$ & Diesel & 39.83 & 1177 & 50.980 & 48.1919 & \\
\hline 1586 & $9 / 2 / 97$ & Diesel & 41.57 & 2246 & 53.210 & 50.3001 & \\
\hline
\end{tabular}




\begin{tabular}{|c|c|c|c|c|c|c|c|}
\hline Truck & Date & Fuel & Amount & Odometer & Cost & Adj. Cost & Monthly Avg \\
\hline 1592 & $9 / 2 / 97$ & Diesel & 30.01 & 137564 & 38.410 & 36.3093 & \\
\hline 1593 & $9 / 2 / 97$ & Diesel & 30.99 & 1427 & 39.670 & 37.5007 & \\
\hline 1586 & $9 / 3 / 97$ & Diesel & 31.27 & 2684 & 40.030 & 37.8411 & \\
\hline 1592 & $9 / 3 / 97$ & Diesel & 32.00 & 137773 & 40.960 & 38.72 & \\
\hline 1593 & $9 / 3 / 97$ & Diesel & 46.36 & 1750 & 59.340 & 56.0948 & \\
\hline 1586 & $9 / 4 / 97$ & Diesel & 36.02 & 2750 & 46.110 & 43.5886 & \\
\hline 1593 & $9 / 4 / 97$ & Diesel & 31.21 & 2009 & 39.950 & 37.7653 & \\
\hline 1592 & $9 / 4 / 97$ & Diesel & 25.43 & 137973 & 32.550 & 30.7699 & \\
\hline 1592 & $9 / 5 / 97$ & Diesel & 44.88 & 138298 & 57.450 & 54.3084 & \\
\hline 1593 & $9 / 5 / 97$ & Diesel & 49.98 & 2303 & 63.970 & 60.4714 & \\
\hline 1586 & $9 / 5 / 97$ & Diesel & 21.98 & 3091 & 28.130 & 26.5914 & \\
\hline 1592 & $9 / 6 / 97$ & Diesel & 30.01 & 138523 & 38.410 & 36.3093 & \\
\hline 1593 & $9 / 6 / 97$ & Diesel & 21.39 & 2500 & 27.380 & 25.8827 & \\
\hline 1586 & $9 / 8 / 97$ & Diesel & 41.49 & 3450 & 53.110 & 50.2057 & \\
\hline 1593 & $9 / 8 / 97$ & Diesel & 30.94 & 2723 & 39.600 & 37.4342 & \\
\hline 1592 & $9 / 9 / 97$ & Diesel & 46.70 & 139050 & 59.780 & 56.511 & \\
\hline 1593 & $9 / 9 / 97$ & Diesel & 47.31 & 3006 & 60.560 & 57.2483 & \\
\hline 1586 & $9 / 10 / 97$ & Diesel & 32.48 & 3795 & 40.600 & 38.3264 & \\
\hline 1592 & $9 / 10 / 97$ & Diesel & 27.00 & 139277 & 33.750 & 31.86 & \\
\hline 1586 & $9 / 11 / 97$ & Diesel & 36.24 & 4066 & 45.300 & 42.7632 & \\
\hline 1592 & $9 / 11 / 97$ & Diesel & 40.02 & 139545 & 50.030 & 47.2286 & \\
\hline 1593 & $9 / 11 / 97$ & Diesel & 41.04 & 3281 & 51.300 & 48.4272 & \\
\hline 1586 & $9 / 12 / 97$ & Diesel & 43.75 & 4398 & 54.690 & 51.6275 & \\
\hline 1592 & $9 / 12 / 97$ & Diesel & 33.85 & 139774 & 42.310 & 39.9405 & \\
\hline 1593 & $9 / 12 / 97$ & Diesel & 39.26 & 3542 & 49.080 & 46.3318 & \\
\hline 1586 & $9 / 13 / 97$ & Diesel & 29.06 & 4633 & 36.330 & 34.2958 & \\
\hline 1592 & $9 / 13 / 97$ & Diesel & 28.30 & 139994 & 35.400 & 33.419 & \\
\hline 1592 & $9 / 15 / 97$ & Diesel & 26.10 & 140201 & 32.660 & 30.833 & \\
\hline 1593 & $9 / 15 / 97$ & Diesel & 42.09 & 3826 & 52.610 & 49.6637 & \\
\hline 1586 & $9 / 16 / 97$ & Diesel & 30.05 & 4875 & 37.260 & 35.1565 & \\
\hline 1592 & $9 / 16 / 97$ & Diesel & 30.40 & 140550 & 37.700 & 35.572 & \\
\hline 1593 & $9 / 16 / 97$ & Diesel & 31.99 & 4100 & 39.670 & 37.4307 & \\
\hline 1586 & $9 / 17 / 97$ & Diesel & 38.74 & 5432 & 48.040 & 45.3282 & \\
\hline 1592 & $9 / 17 / 97$ & Diesel & 32.08 & 140620 & 39.780 & 37.5344 & \\
\hline 1593 & $9 / 17 / 97$ & Diesel & 37.14 & 4294 & 46.050 & 43.4502 & \\
\hline 1586 & $9 / 18 / 97$ & Diesel & 36.44 & 5700 & 45.190 & 42.6392 & \\
\hline 1592 & $9 / 18 / 97$ & Diesel & 28.63 & 140835 & 35.500 & 33.4959 & \\
\hline 1593 & $9 / 18 / 97$ & Diesel & 32.18 & 4533 & 39.900 & 37.6474 & \\
\hline 1586 & $9 / 19 / 97$ & Diesel & 16.29 & 5846 & 20.200 & 19.0597 & \\
\hline 1592 & $9 / 19 / 97$ & Diesel & 31.15 & 141066 & 38.630 & 36.4495 & \\
\hline 1586 & $9 / 20 / 97$ & Diesel & 8.98 & 5896 & 11.140 & 10.5114 & \\
\hline 1592 & $9 / 20 / 97$ & Diesel & 24.99 & 141279 & 30.990 & 29.2407 & \\
\hline 1593 & $9 / 20 / 97$ & Diesel & 27.27 & 4976 & 33.810 & 31.9011 & \\
\hline 1586 & $9 / 22 / 97$ & Diesel & 23.20 & 6068 & 29.000 & 27.376 & \\
\hline 1592 & $9 / 22 / 97$ & Diesel & 30.42 & 141485 & 38.030 & 35.9006 & \\
\hline 1593 & $9 / 22 / 97$ & Diesel & 28.89 & 5226 & 36.110 & 34.0877 & \\
\hline 1586 & $9 / 23 / 97$ & Diesel & 34.29 & 6295 & 42.860 & 40.4597 & \\
\hline 1593 & $9 / 23 / 97$ & Diesel & 36.29 & 5488 & 45.360 & 42.8197 & \\
\hline 1586 & $9 / 24 / 97$ & Diesel & 27.57 & 6556 & 34.460 & 32.5301 & \\
\hline 1592 & $9 / 24 / 97$ & Diesel & 60.05 & 141931 & 75.060 & 70.8565 & \\
\hline 1593 & $9 / 24 / 97$ & Diesel & 24.00 & 5651 & 30.000 & 28.32 & \\
\hline 1586 & $9 / 25 / 97$ & Diesel & 46.88 & 6847 & 58.130 & 54.8484 & \\
\hline 1592 & $9 / 25 / 97$ & Diesel & 30.37 & 142143 & 37.660 & 35.5341 & \\
\hline 1593 & $9 / 25 / 97$ & Diesel & 37.84 & 5909 & 46.920 & 44.2712 & \\
\hline
\end{tabular}




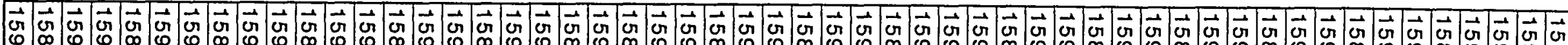

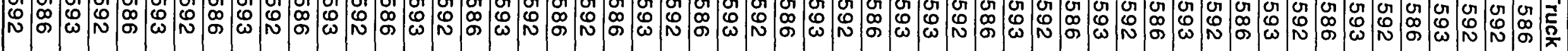

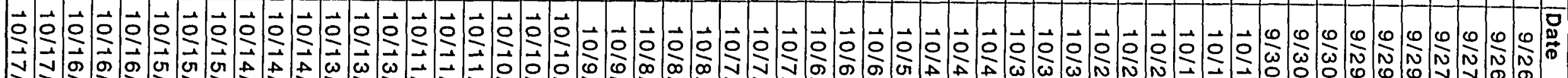

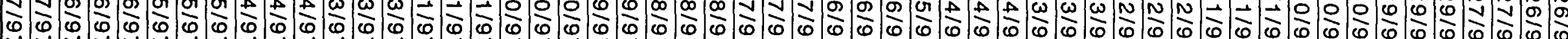

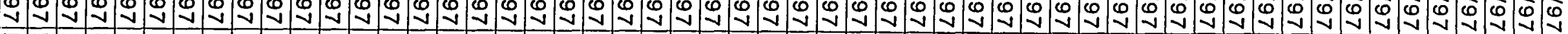

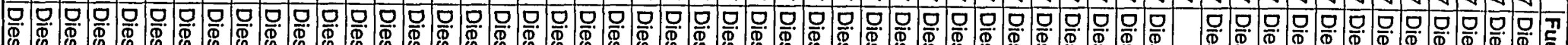

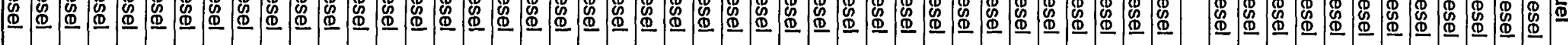

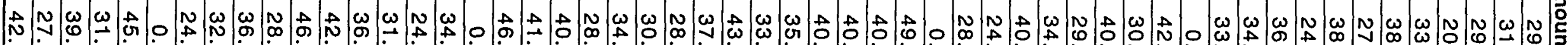

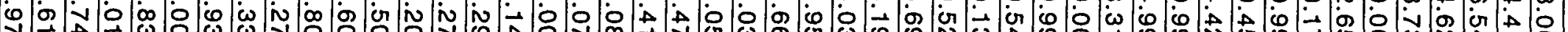

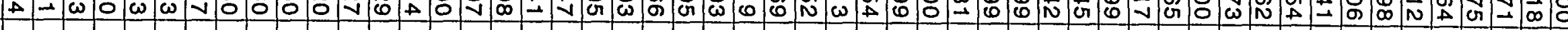

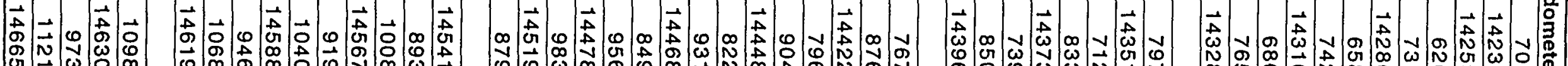

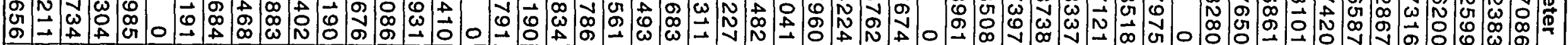

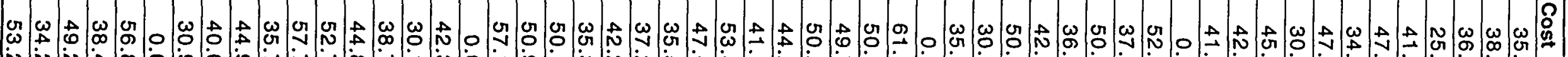

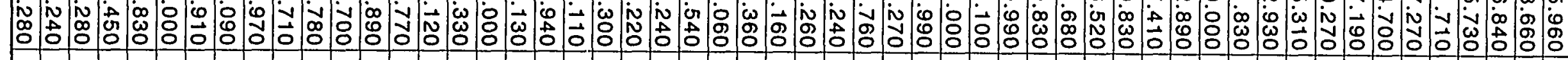

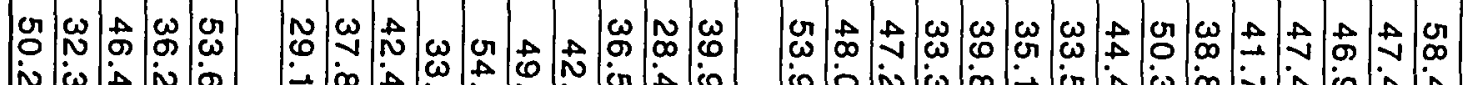

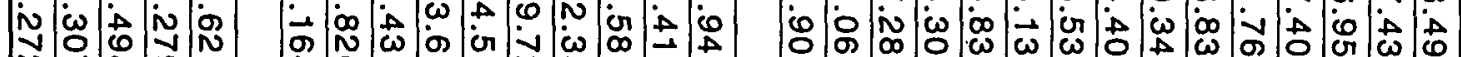

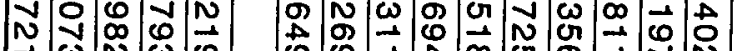

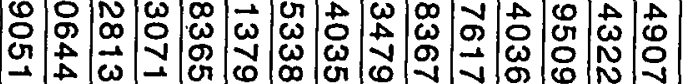

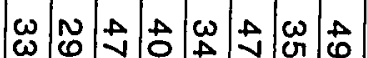

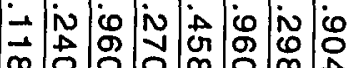

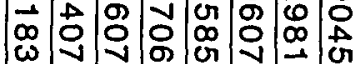

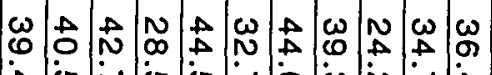

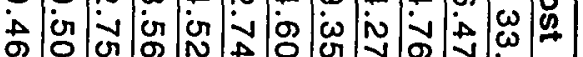

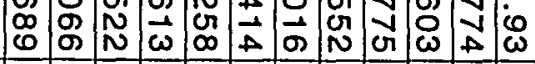




\begin{tabular}{|c|c|c|c|c|c|c|c|}
\hline Truck & Date & Fuel & Amount & Odometer & Cost & Adj. Cost & Monthly Avg \\
\hline 1593 & $10 / 17 / 97$ & Diesel & 39.94 & 10020 & 49.530 & 46.7342 & \\
\hline 1586 & $10 / 18 / 97$ & Diesel & 21.95 & 11378 & 27.220 & 25.6835 & \\
\hline 1592 & $10 / 18 / 97$ & Diesel & 27.99 & 146880 & 34.710 & 32.7507 & \\
\hline 1593 & $10 / 18 / 97$ & Diesel & 35.91 & 10286 & 44.530 & 42.0163 & \\
\hline 1586 & $10 / 20 / 97$ & Diesel & 34.92 & 11630 & 43.300 & 40.8556 & \\
\hline 1592 & $10 / 20 / 97$ & Diesel & 35.99 & 147152 & 44.630 & 42.1107 & \\
\hline 1593 & $10 / 20 / 97$ & Diesel & 34.29 & 10523 & 42.520 & 40.1197 & \\
\hline 1586 & $10 / 21 / 97$ & Diesel & 31.31 & 11872 & 38.820 & 36.6283 & \\
\hline 1592 & $10 / 21 / 97$ & Diesel & 29.31 & 147362 & 36.340 & 34.2883 & \\
\hline 1593 & $10 / 21 / 97$ & Diesel & 35.00 & $10760^{\circ}$ & 43.400 & 40.95 & \\
\hline 1586 & $10 / 22 / 97$ & Diesel & 33.85 & 12100 & 41.970 & 39.6005 & \\
\hline 1592 & $10 / 22 / 97$ & Diesel & 31.49 & 147571 & 39.050 & 36.8457 & \\
\hline 1593 & $10 / 22 / 97$ & Diesel & 0.00 & 0 & 0.000 & & \\
\hline 1586 & $10 / 23 / 97$ & Diesel & 40.99 & 12394 & 51.240 & 48.3707 & \\
\hline 1592 & $10 / 23 / 97$ & Diesel & 24.39 & 147875 & 30.490 & 28.7827 & \\
\hline 1593 & $10 / 23 / 97$ & Diesel & 39.33 & 11034 & 49.160 & 46.4069 & \\
\hline 1586 & $10 / 24 / 97$ & Diesel & 37.18 & 12628 & 46.480 & 43.8774 & \\
\hline 1592 & $10 / 24 / 97$ & Diesel & 63.10 & 148181 & 78.880 & 74.463 & \\
\hline 1593 & $10 / 24 / 97$ & Diesel & 45.47 & 11311 & 56.840 & 53.6571 & \\
\hline 1586 & $10 / 25 / 97$ & Diesel & 22.05 & 12799 & 27.560 & 26.0165 & \\
\hline 1592 & $10 / 25 / 97$ & Diesel & 28.00 & 148413 & 35.000 & 33.04 & \\
\hline 1593 & $10 / 25 / 97$ & Diesel & 33.61 & 11548 & 42.010 & 39.6573 & \\
\hline 1586 & $10 / 27 / 97$ & Diesel & 37.93 & 13052 & 47.410 & 44.7549 & \\
\hline 1592 & $10 / 27 / 97$ & Diesel & 39.02 & 148699 & 48.780 & 46.0486 & \\
\hline 1593 & $10 / 27 / 97$ & Diesel & 36.39 & 11800 & 45.490 & 42.9427 & \\
\hline 1586 & $10 / 28 / 97$ & Diesel & 30.07 & 13250 & 39.090 & 36.9851 & \\
\hline 1592 & $10 / 28 / 97$ & Diesel & 19.16 & 148900 & 24.910 & 23.5688 & \\
\hline 1593 & $10 / 28 / 97$ & Diesel & 34.49 & 12062 & 44.840 & 42.4257 & \\
\hline 1586 & $10 / 29 / 97$ & Diesel & 35.69 & 13555 & 46.400 & 43.9017 & \\
\hline 1592 & $10 / 29 / 97$ & Diesel & 30.53 & 149103 & 39.690 & 37.5529 & \\
\hline 1593 & $10 / 29 / 97$ & Diesel & 22.12 & 12200 & 28.760 & 27.2116 & \\
\hline 1586 & $10 / 30 / 97$ & Diesel & 39.19 & 13818 & 50.950 & 48.2067 & \\
\hline 1592 & $10 / 30 / 97$ & Diesel & 36.18 & 149361 & 47.040 & 44.5074 & \\
\hline 1593 & $10 / 30 / 97$ & Diesel & 31.28 & 12420 & 40.660 & 38.4704 & \\
\hline 1586 & $10 / 31 / 97$ & Diesel & 0.00 & 0 & 0.000 & & \\
\hline 1592 & $10 / 31 / 97$ & Diesel & 36.00 & 149573 & 46.800 & 44.28 & \\
\hline 1593 & $10 / 31 / 97$ & Diesel & 30.49 & 12650 & 39.640 & 37.5057 & 1.179634435 \\
\hline 1586 & $11 / 1 / 97$ & Diesel & 23.01 & 13973 & 29.910 & 28.2993 & \\
\hline 1592 & $11 / 1 / 97$ & Diesel & 33.26 & 149777 & 43.240 & 40.9118 & \\
\hline 1593 & $11 / 1 / 97$ & Diesel & 0.00 & 0 & 0.000 & & \\
\hline 1586 & $11 / 3 / 97$ & Diesel & 45.06 & 14294 & 61.280 & 58.1258 & \\
\hline 1592 & $11 / 3 / 97$ & Diesel & 34.16 & 150027 & 46.460 & 44.0688 & \\
\hline 1593 & $11 / 3 / 97$ & Diesel & 43.80 & 12917 & 59.570 & 56.504 & \\
\hline 1586 & $11 / 4 / 97$ & Diesel & 36.53 & 14583 & 49.680 & 47.1229 & \\
\hline 1593 & $11 / 4 / 97$ & Diesel & 41.36 & 13200 & 56.250 & 53.3548 & \\
\hline 1586 & $11 / 5 / 97$ & Diesel & 36.57 & 14485 & 49.740 & 47.1801 & \\
\hline 1592 & $11 / 5 / 97$ & Diesel & 32.75 & 150496 & 44.540 & 42.2475 & \\
\hline 1593 & $11 / 5 / 97$ & Diesel & 30.00 & 13454 & 40.800 & 38.7 & \\
\hline 1593 & $11 / 5 / 97$ & Diesel & 40.63 & 13757 & 55.260 & 52.4159 & \\
\hline 1586 & $11 / 6 / 97$ & Diesel & 33.65 & 15092 & 45.760 & 43.4045 & \\
\hline 1592 & $11 / 6 / 97$ & Diesel & 30.38 & 150700 & 41.320 & 39.1934 & \\
\hline 1593 & $11 / 6 / 97$ & Diesel & 57.48 & 14097 & 78.170 & 74.1464 & \\
\hline 1586 & $11 / 7 / 97$ & Diesel & 37.97 & 15368 & 51.640 & 48.9821 & \\
\hline 1592 & $11 / 7 / 97$ & Diesel & 28.20 & 150932 & 38.350 & 36.376 & \\
\hline
\end{tabular}




\begin{tabular}{|c|c|c|c|c|c|c|c|}
\hline Truck & Date & Fuel & Amount & Odometer & Cost & Adj. Cost & Monthly Avg \\
\hline 1593 & $11 / 7 / 97$ & Diesel & 45.31 & 14424 & 61.620 & 58.4483 & \\
\hline 1586 & $11 / 8 / 97$ & Diesel & 26.48 & 15620 & 36.010 & 34.1564 & \\
\hline 1592 & $11 / 8 / 97$ & Diesel & 24.97 & 151150 & 33.960 & 32.2121 & \\
\hline 1586 & $11 / 10 / 97$ & Diesel & 43.19 & 15818 & 58.740 & 55.7167 & \\
\hline 1592 & $11 / 10 / 97$ & Diesel & 40.26 & 151370 & 54.750 & 51.9318 & \\
\hline 1593 & $11 / 10 / 97$ & Diesel & 41.53 & 14673 & 56.480 & 53.5729 & \\
\hline 1586 & $11 / 11 / 97$ & Diesel & 32.01 & 16054 & 43.530 & 41.2893 & \\
\hline 1592 & $11 / 11 / 97$ & Diesel & 22.69 & 151578 & 30.860 & 29.2717 & \\
\hline 1593 & $11 / 11 / 97$ & Diesel & 32.95 & 14930 & 44.810 & 42.5035 & \\
\hline 1586 & $11 / 12 / 97$ & Diesel & 42.25 & 16336 & 55.770 & 52.8125 & \\
\hline 1592 & $11 / 12 / 97$ & Diesel & 33.28 & 151800 & 43.930 & 41.6004 & \\
\hline 1593 & $11 / 12 / 97$ & Diesel & 28.08 & 15108 & 37.070 & 35.1044 & \\
\hline 1586 & $11 / 13 / 97$ & Diesel & 42.64 & 16608 & 56.280 & 53.2952 & \\
\hline 1592 & $11 / 13 / 97$ & Diesel & 30.28 & 152006 & 39.970 & 37.8504 & \\
\hline 1593 & $11 / 13 / 97$ & Diesel & 35.79 & 15361 & 47.240 & 44.7347 & \\
\hline 1586 & $11 / 14 / 97$ & Diesel & 0.00 & 0 & 0.000 & & \\
\hline 1592 & $11 / 14 / 97$ & Diesel & 27.98 & 212 & 36.930 & 34.9714 & \\
\hline 1593 & $11 / 14 / 97$ & Diesel & 46.93 & 15642 & 61.950 & 58.6649 & \\
\hline 1586 & $11 / 15 / 97$ & Diesel & 43.04 & 16911 & 56.810 & 53.7972 & \\
\hline 1592 & $11 / 15 / 97$ & Diesel & 35.03 & 437 & 46.240 & 43.7879 & \\
\hline 1593 & $11 / 15 / 97$ & Diesel & 52.00 & 15996 & 68.640 & 65 & \\
\hline 1586 & $11 / 17 / 97$ & Diesel & 19.99 & 17106 & 26.390 & 24.9907 & \\
\hline 1592 & $11 / 17 / 97$ & Diesel & 35.02 & 707 & 46.230 & 43.7786 & \\
\hline 1593 & $11 / 17 / 97$ & Diesel & 36.01 & 16243 & 47.530 & 45.0093 & \\
\hline 1586 & $11 / 18 / 97$ & Diesel & 36.96 & 17338 & 46.200 & 43.6128 & \\
\hline 1592 & $11 / 18 / 97$ & Diesel & 25.54 & 919 & 31.930 & 30.1422 & \\
\hline 1593 & $11 / 18 / 97$ & Diesel & 32.05 & 16525 & 40.060 & 37.8165 & \\
\hline 1586 & $11 / 19 / 97$ & Diesel & 32.63 & 17559 & 40.790 & 38.5059 & \\
\hline 1592 & $11 / 19 / 97$ & Diesel & 34.42 & 1157 & 43.030 & 40.6206 & \\
\hline 1593 & $11 / 19 / 97$ & Diesel & 52.73 & 16889 & 65.910 & 62.2189 & \\
\hline 1586 & $11 / 20 / 97$ & Diesel & 40.81 & 17798 & 51.010 & 48.1533 & \\
\hline 1592 & $11 / 20 / 97$ & Diesel & 33.30 & 1363 & 41.630 & 39.299 & \\
\hline 1593 & $11 / 20 / 97$ & Diesel & 35.64 & 17120 & 44.550 & 42.0552 & \\
\hline 1586 & $11 / 21 / 97$ & Diesel & 37.09 & 18066 & 46.360 & 43.7637 & \\
\hline 1592 & $11 / 21 / 97$ & Diesel & 44.20 & 1644 & 55.230 & 52.136 & \\
\hline 1593 & $11 / 21 / 97$ & Diesel & 42.90 & 17410 & 53.630 & 50.627 & \\
\hline 1586 & $11 / 22 / 97$ & Diesel & 16.99 & 18180 & 21.240 & 20.0507 & \\
\hline 1593 & $11 / 22 / 97$ & Diesel & 39.00 & 17675 & 48.750 & 46.02 & \\
\hline 1592 & $11 / 22 / 97$ & Diesel & 17.97 & 1706 & 22.460 & 21.2021 & \\
\hline 1586 & $11 / 24 / 97$ & Diesel & 37.61 & 18436 & 46.640 & 44.0073 & \\
\hline 1592 & $11 / 24 / 97$ & Diesel & 31.40 & 1850 & 38.940 & 36.742 & \\
\hline 1593 & $11 / 24 / 97$ & Diesel & 31.11 & 17925 & 38.580 & 36.4023 & \\
\hline 1586 & $11 / 25 / 97$ & Diesel & 37.28 & 18703 & 45.480 & 42.8704 & \\
\hline 1593 & $11 / 25 / 97$ & Diesel & 42.78 & 18200 & 52.190 & 49.1954 & \\
\hline 1592 & $11 / 25 / 97$ & Diesel & 0.00 & 0 & 0.000 & & \\
\hline 1592 & $11 / 26 / 97$ & Diesel & 39.43 & 2038 & 47.710 & 44.9499 & \\
\hline 1593 & $11 / 26 / 97$ & Diesel & 35.01 & 18429 & 42.360 & 39.9093 & \\
\hline 1586 & $11 / 26 / 97$ & Diesel & 0.00 & 0 & 0.000 & & \\
\hline 1586 & $11 / 27 / 97$ & Diesel & 0.00 & 0 & 0.000 & & \\
\hline 1592 & $11 / 27 / 97$ & Diesel & 0.00 & 0 & 0.000 & & \\
\hline 1593 & $11 / 27 / 97$ & Diesel & 0.00 & 0 & 0.000 & & \\
\hline 1586 & $11 / 28 / 97$ & Diesel & 34.98 & 18964 & 42.330 & 39.8814 & \\
\hline 1592 & $11 / 28 / 97$ & Diesel & 34.49 & 2549 & 41.730 & 39.3157 & \\
\hline 1593 & $11 / 28 / 97$ & Diesel & 31.99 & 18678 & 38.710 & 36.4707 & \\
\hline
\end{tabular}




\begin{tabular}{|c|c|c|c|c|c|c|c|}
\hline Truck & Date & Fuel & Amount & Odometer & Cost & Adj. Cost & Monthly Avg \\
\hline 1586 & $11 / 29 / 97$ & Diesel & 24.93 & 19131 & 30.170 & 28.4249 & \\
\hline 1592 & $11 / 29 / 97$ & Diesel & 24.98 & 2766 & 30.230 & 28.4814 & \\
\hline 1593 & $11 / 29 / 97$ & Diesel & 42.17 & 18948 & 51.030 & 48.0781 & 1.23 \\
\hline 1586 & $12 / 1 / 97$ & Diesel & 47.53 & 19455 & 57.510 & 54.1829 & \\
\hline 1592 & $12 / 1 / 97$ & Diesel & 29.65 & 2969 & 35.880 & 33.8045 & \\
\hline 1593 & $12 / 1 / 97$ & Diesel & 34.67 & 19206 & 41.950 & 39.5231 & \\
\hline 1586 & $12 / 2 / 97$ & Diesel & 0.00 & 0 & 0.000 & & \\
\hline 1592 & $12 / 2 / 97$ & Diesel & 31.57 & 3175 & 38.200 & 35.9901 & \\
\hline 1593 & $12 / 2 / 97$ & Diesel & 41.17 & 19498 & 49.820 & 46.9381 & \\
\hline 1586 & $12 / 3 / 97$ & Diesel & 0.00 & 0 & 0.000 & & \\
\hline 1586 & $12 / 3 / 97$ & Diesel & 13.54 & 19452 & 15.160 & 15.160 & \\
\hline 1592 & $12 / 3 / 97$ & Diesel & 28.01 & 3386 & 31.370 & 31.370 & \\
\hline 1593 & $12 / 3 / 97$ & Diesel & 0.00 & 0 & 0.000 & 0.000 & \\
\hline 1586 & $12 / 4 / 97$ & Diesel & 29.26 & 19754 & 32.770 & 32.770 & \\
\hline 1592 & $12 / 4 / 97$ & Diesel & 35.91 & 3672 & 40.220 & 40.220 & \\
\hline 1593 & $12 / 4 / 97$ & Diesel & 41.42 & 19763 & 46.390 & 46.390 & \\
\hline 1586 & $12 / 5 / 97$ & Diesel & 65.71 & 20145 & 73.600 & 73.600 & \\
\hline 1592 & $12 / 5 / 97$ & Diesel & 33.52 & 3883 & 37.540 & 37.540 & \\
\hline 1593 & $12 / 5 / 97$ & Diesel & 37.01 & 20013 & 41.450 & 41.450 & \\
\hline 1586 & $12 / 6 / 97$ & Diesel & 46.30 & 20475 & 51.860 & 51.860 & \\
\hline 1592 & $12 / 6 / 97$ & Diesel & 26.01 & 4100 & 29.130 & 29.130 & \\
\hline 1593 & $12 / 6 / 97$ & Diesel & 42.20 & 20323 & 47.260 & 47.260 & \\
\hline 1586 & $12 / 8 / 97$ & Diesel & 29.73 & 20688 & 33.300 & 33.300 & \\
\hline 1592 & $12 / 8 / 97$ & Diesel & 41.09 & 4361 & 46.020 & 46.020 & \\
\hline 1593 & $12 / 8 / 97$ & Diesel & 41.38 & 20577 & 46.350 & 46.350 & \\
\hline 1586 & $12 / 9 / 97$ & Diesel & 30.99 & 20880 & 34.710 & 34.710 & \\
\hline 1592 & $12 / 9 / 97$ & Diesel & 28.69 & 4570 & 32.130 & 32.130 & \\
\hline 1593 & $12 / 9 / 97$ & Diesel & 42.44 & 20837 & 47.530 & 47.530 & \\
\hline 1586 & $12 / 10 / 97$ & Diesel & 72.35 & 21390 & 81.030 & 81.030 & \\
\hline 1592 & $12 / 10 / 97$ & Diesel & 29.35 & 4780 & 32.870 & 32.870 & \\
\hline 1593 & $12 / 10 / 97$ & Diesel & 0.00 & 0 & 0.000 & 0.000 & \\
\hline 1586 & $12 / 11 / 97$ & Diesel & 24.01 & 21579 & 26.890 & 26.890 & \\
\hline 1592 & $12 / 11 / 97$ & Diesel & 32.47 & 5016 & 36.370 & 36.370 & \\
\hline 1593 & $12 / 11 / 97$ & Diesel & 32.38 & 21071 & 36.270 & 36.270 & \\
\hline 1586 & $12 / 12 / 97$ & Diesel & 56.99 & 21967 & 63.830 & 63.830 & \\
\hline 1592 & $12 / 12 / 97$ & Diesel & 33.76 & 5246 & 37.810 & 37.810 & \\
\hline 1593 & $12 / 12 / 97$ & Diesel & 42.96 & 21348 & 48.120 & 48.120 & \\
\hline 1586 & $12 / 13 / 97$ & Diesel & 26.35 & 22150 & 29.510 & 29.510 & \\
\hline 1592 & $12 / 13 / 97$ & Diesel & 26.89 & 5452 & 30.120 & 30.120 & \\
\hline 1593 & $12 / 13 / 97$ & Diesel & 41.35 & 21644 & 46.310 & 46.310 & \\
\hline 1592 & $12 / 15 / 97$ & Diesel & 28.62 & 5652 & 32.050 & 32.050 & \\
\hline 1586 & $12 / 16 / 97$ & Diesel & 71.38 & 22615 & 79.950 & 79.950 & \\
\hline 1592 & $12 / 16 / 97$ & Diesel & 25.90 & 5852 & 29.010 & 29.010 & \\
\hline 1593 & $12 / 16 / 97$ & Diesel & 37.89 & 21895 & 42.440 & 42.440 & \\
\hline 1586 & $12 / 17 / 97$ & Diesel & 73.92 & 23124 & 82.790 & 82.790 & \\
\hline 1592 & $12 / 17 / 97$ & Diesel & 32.00 & 6059 & 35.840 & 35.840 & \\
\hline 1593 & $12 / 17 / 97$ & Diesel & 18.02 & 22024 & 20.180 & 20.180 & \\
\hline 1586 & $12 / 18 / 97$ & Diesel & 23.51 & 23288 & 25.390 & 25.390 & \\
\hline 1592 & $12 / 18 / 97$ & Diesel & 26.29 & 6264 & 28.390 & 28.390 & \\
\hline 1593 & $12 / 18 / 97$ & Diesel & 34.56 & 22256 & 37.320 & 37.320 & \\
\hline 1586 & $12 / 19 / 97$ & Diesel & 55.99 & 23673 & 60.470 & 60.470 & \\
\hline 1592 & $12 / 19 / 97$ & Diese! & 33.03 & 6513 & 35.670 & 35.670 & \\
\hline 1593 & $12 / 19 / 97$ & Diesel & 39.13 & 22533 & 42.260 & 42.260 & \\
\hline 1586 & $12 / 20 / 97$ & Diesel & 27.04 & 23877 & 29.200 & 29.200 & \\
\hline
\end{tabular}




\begin{tabular}{|c|c|c|c|c|c|c|c|}
\hline Truck & Date & Fuel & Amount & Odometer & Cost & Adj. Cost & Monthly Avg \\
\hline 1592 & $12 / 20 / 97$ & Diesel & 25.21 & 6726 & 27.230 & 27.230 & \\
\hline 1593 & $12 / 20 / 97$ & Diesel & 28.81 & 22729 & 31.110 & 31.110 & \\
\hline 1592 & $12 / 22 / 97$ & Diesel & 39.27 & 6955 & 42.410 & 42.410 & \\
\hline 1593 & $12 / 22 / 97$ & Diesel & 41.62 & 22979 & 44.950 & 44.950 & \\
\hline 1586 & $12 / 22 / 97$ & Diesel & 0.00 & 0 & 0.000 & 0.000 & \\
\hline 1586 & $12 / 23 / 97$ & Diesel & 70.38 & 24309 & 76.010 & 76.010 & \\
\hline 1592 & $12 / 23 / 97$ & Diesel & 29.81 & 7163 & 32.190 & 32.190 & \\
\hline 1593 & $12 / 23 / 97$ & Diesel & 42.53 & 23257 & 45.930 & 45.930 & \\
\hline 1586 & $12 / 24 / 97$ & Diesel & 67.16 & 24846 & 73.200 & 73.200 & \\
\hline 1592 & $12 / 24 / 97$ & Diesel & 38.27 & 7438 & 41.710 & 41.710 & \\
\hline 1593 & $12 / 24 / 97$ & Diesel & 24.02 & 23429 & 26.180 & 26.180 & \\
\hline 1592 & $12 / 26 / 97$ & Diesel & 32.73 & 7651 & 35.680 & 35.680 & \\
\hline 1593 & $12 / 26 / 97$ & Diesel & 36.78 & 23685 & 40.090 & 40.090 & \\
\hline 1586 & $12 / 26 / 97$ & Diesel & 0.00 & 0 & 0.000 & 0.000 & \\
\hline 1592 & $12 / 27 / 97$ & Diesel & 25.01 & 7895 & 27.260 & 27.260 & \\
\hline 1593 & $12 / 27 / 97$ & Diesel & 19.25 & 23901 & 20.980 & 20.980 & \\
\hline 1586 & $12 / 27 / 97$ & Diesel & 71.02 & 25400 & 77.410 & 77.410 & \\
\hline 1592 & $12 / 29 / 97$ & Diesel & 43.61 & 8159 & 47.530 & 47.530 & \\
\hline 1593 & $12 / 29 / 97$ & Diesel & 46.00 & 24166 & 50.140 & 50.140 & \\
\hline 1586 & $12 / 29 / 97$ & Diesel & 0.00 & 0 & 0.000 & 0.000 & \\
\hline 1586 & $12 / 30 / 97$ & Diesel & 67.04 & 25796 & 73.070 & 73.070 & \\
\hline 1592 & $12 / 30 / 97$ & Diesel & 31.19 & 8408 & 34.000 & 34.000 & \\
\hline 1593 & $12 / 30 / 97$ & Diesel & 33.98 & 24402 & 37.040 & 37.040 & \\
\hline 1593 & $12 / 30 / 97$ & Diesel & 28.01 & 24611 & 30.530 & 30.530 & \\
\hline 1586 & $12 / 31 / 97$ & Diesel & 61.63 & 26304 & 67.500 & 67.500 & \\
\hline 1592 & $12 / 31 / 97$ & Diesel & 24.94 & 8616 & 27.180 & 27.180 & 1.11 \\
\hline & & Totals & 1371727 & & & 1500801 & \\
\hline & & & & & Average & 1.17 & \\
\hline & & & & & & & \\
\hline \multicolumn{8}{|c|}{ Note: Fuel cost through $12 / 2 / 97$ had $\$ 0.07$ added as a surcharge } \\
\hline \multicolumn{8}{|c|}{ for another fleet buying fuel from Raley's. This surcharge has } \\
\hline been $r$ & moved for $t$ & his $\cos$ & er gallon & & & & \\
\hline
\end{tabular}




\begin{tabular}{|c|c|c|c|c|c|c|}
\hline Truck & Date & Fuel & Amount & Odometer & Cost & Monthly Avg \\
\hline 1586 & $1 / 2 / 98$ & Diesel & 63.06 & 26682.0 & 70.00 & \\
\hline 1592 & $1 / 2 / 98$ & Diesel & 33.01 & 8818.0 & 36.64 & \\
\hline 1593 & $1 / 2 / 98$ & Diesel & 0.00 & 0.0 & 0.00 & \\
\hline 1586 & $1 / 3 / 98$ & Diesel & 20.04 & 26810.0 & 22.24 & \\
\hline 1592 & $1 / 3 / 98$ & Diesel & 0.00 & 0.0 & 0.00 & \\
\hline 1593 & $1 / 3 / 98$ & Diesel & 24.30 & 25078.0 & 26.97 & \\
\hline 1586 & $1 / 5 / 98$ & Diesel & 0.00 & 0.0 & 0.00 & \\
\hline 1592 & $1 / 5 / 98$ & Diesel & 32.30 & 9359.0 & 35.85 & \\
\hline 1593 & $1 / 5 / 98$ & Diesel & 52.27 & 25356.0 & 58.02 & \\
\hline 1586 & $1 / 6 / 98$ & Diesel & 65.00 & 27080.0 & 72.15 & \\
\hline 1592 & $1 / 6 / 98$ & Diesel & 27.91 & 9563.0 & 30.98 & \\
\hline 1593 & $1 / 6 / 98$ & Diesel & 45.16 & 25638.0 & 50.13 & \\
\hline 1586 & $1 / 7 / 98$ & Diesel & 72.22 & 27233.0 & 80.16 & \\
\hline 1592 & $1 / 7 / 98$ & Diesel & 34.83 & 9708.0 & 38.66 & \\
\hline 1586 & $1 / 8 / 98$ & Diesel & 50.83 & 28065.0 & 55.91 & \\
\hline 1592 & $1 / 8 / 98$ & Diesel & 24.56 & 9982.0 & 27.02 & \\
\hline 1593 & $1 / 8 / 98$ & Diesel & 36.29 & 25878.0 & 39.92 & \\
\hline 1.586 & $1 / 9 / 98$ & Diesel & 50.30 & 28366.0 & 55.33 & \\
\hline 1592 & $1 / 9 / 98$ & Diesel & 14.26 & 10073.0 & 15.69 & \\
\hline 1593 & $1 / 9 / 98$ & Diesel & 35.78 & 26145.0 & 39.36 & \\
\hline 1586 & $1 / 10 / 98$ & Diesel & 33.01 & 28598.0 & 36.31 & \\
\hline 1592 & $1 / 10 / 98$ & Diesel & 28.63 & 10283.0 & 31.49 & \\
\hline 1586 & $1 / 12 / 98$ & Diesel & 50.27 & 28881.0 & 55.30 & \\
\hline 1592 & $1 / 12 / 98$ & Diesel & 35.38 & 10554.0 & 38.92 & \\
\hline 1593 & $1 / 12 / 98$ & Diesel & 39.46 & 26400.0 & 43.41 & \\
\hline 1586 & $1 / 13 / 98$ & Diesel & 34.28 & 29123.0 & 37.71 & \\
\hline 1592 & $1 / 13 / 98$ & Diesel & 27.25 & 10763.0 & 29.98 & \\
\hline 1593 & $1 / 13 / 98$ & Diesel & 42.53 & 26681.0 & 46.78 & \\
\hline 1586 & $1 / 14 / 98$ & Diesel & 77.82 & 29297.0 & 85.60 & \\
\hline 1592 & $1 / 14 / 98$ & Diesel & 31.37 & 11013.0 & 34.51 & \\
\hline 1592 & $1 / 15 / 98$ & Diesel & 24.21 & 11272.0 & 26.63 & \\
\hline 1593 & $1 / 15 / 98$ & Diesel & 33.94 & 26952.0 & 37.33 & \\
\hline 1586 & $1 / 16 / 98$ & Diesel & 83.02 & 30211.0 & 89.66 & \\
\hline 1592 & $1 / 16 / 98$ & Diesel & 32.81 & 11505.0 & 35.43 & \\
\hline 1593 & $1 / 16 / 98$ & Diesel & 36.02 & 27189.0 & 38.90 & \\
\hline 1586 & $1 / 17 / 98$ & Diesel & 22.75 & 30376.0 & 24.57 & \\
\hline 1592 & $1 / 17 / 98$ & Diesel & 29.28 & 11595.0 & 31.62 & \\
\hline 1586 & $1 / 19 / 98$ & Diesel & 34.01 & 30597.0 & 36.73 & \\
\hline 1592 & $1 / 19 / 98$ & Diesel & 36.48 & 11854.0 & 39.40 & \\
\hline 1593 & $1 / 19 / 98$ & Diesel & 45.01 & 27436.0 & 48.61 & \\
\hline 1592 & $1 / 20 / 98$ & Diesel & 27.86 & 12062.0 & 30.09 & \\
\hline 1593 & $1 / 20 / 98$ & Diesel & 44.29 & 27779.0 & 47.83 & \\
\hline 1586 & $1 / 20 / 98$ & Diesel & 32.21 & 30805.0 & 34.79 & \\
\hline 1592 & $1 / 21 / 98$ & Diesel & 21.33 & 12267.0 & 23.04 & \\
\hline 1586 & $1 / 21 / 98$ & Diesel & 69.59 & 31100.0 & 75.16 & \\
\hline 1592 & $1 / 22 / 98$ & Diesel & 29.92 & 12494.0 & 32.31 & \\
\hline 1593 & $1 / 22 / 98$ & Diesel & 35.15 & 28011.0 & 37.96 & \\
\hline 1586 & $1 / 22 / 98$ & Diesel & 62.24 & 31500.0 & 67.22 & \\
\hline 1586 & $1 / 23 / 98$ & Diesel & 70.91 & 31825.0 & 76.58 & \\
\hline 1592 & $1 / 23 / 98$ & Diesel & 33.62 & 12755.0 & 36.31 & \\
\hline 1593 & $1 / 23 / 98$ & Diesel & 32.34 & 28257.0 & 34.93 & \\
\hline 1592 & $1 / 24 / 98$ & Diesel & 34.99 & 12961.0 & 37.79 & \\
\hline 1593 & $1 / 24 / 98$ & Diesel & 45.56 & 28510.0 & 49.20 & \\
\hline 1586 & $1 / 26 / 98$ & Diesel & 27.17 & 32034.0 & 28.80 & \\
\hline
\end{tabular}




\begin{tabular}{|c|c|c|c|c|c|c|}
\hline Truck & Date & Fuel & Amount & Odometer & Cost & Monthly Avg \\
\hline 1592 & $1 / 26 / 98$ & Diesel & 31.92 & 13183.0 & 33.84 & \\
\hline 1593 & $1 / 26 / 98$ & Diesel & 40.23 & 28802.0 & 42.64 & \\
\hline 1586 & $1 / 27 / 98$ & Diesel & 25.95 & 32212.0 & 27.51 & \\
\hline 1592 & $1 / 27 / 98$ & Diesel & 29.91 & 13398.0 & 31.70 & \\
\hline 1593 & $1 / 27 / 98$ & Diesel & 41.13 & 29059.0 & 43.60 & \\
\hline 1586 & $1 / 28 / 98$ & Diesel & 31.18 & 32469.0 & 33.05 & \\
\hline 1592 & $1 / 28 / 98$ & Diesel & 20.38 & 13608.0 & 21.60 & \\
\hline 1592 & $1 / 29 / 98$ & Diesel & 36.03 & 13854.0 & 38.19 & \\
\hline 1593 & $1 / 29 / 98$ & Diesel & 32.72 & 29311.0 & 34.68 & \\
\hline 1586 & $1 / 30 / 98$ & Diesel & 68.34 & 32978.0 & 72.44 & \\
\hline 1592 & $1 / 30 / 98$ & Diesel & 27.16 & 14079.0 & 28.79 & \\
\hline 1586 & $1 / 31 / 98$ & Diesel & 30.98 & 33195.0 & 32.84 & \\
\hline 1592 & $1 / 31 / 98$ & Diesel & 31.76 & 14281.0 & 33.67 & 1.09 \\
\hline 1586 & $2 / 2 / 98$ & Diesel & 30.51 & 33407.0 & 32.34 & \\
\hline 1592 & $2 / 2 / 98$ & Diesel & 31.58 & 14507.0 & 33.47 & \\
\hline 1593 & $2 / 2 / 98$ & Diesel & 51.69 & 29594.0 & 54.79 & \\
\hline 1593 & $2 / 2 / 98$ & Diesel & 39.57 & 29846.0 & 41.94 & \\
\hline 1586 & $2 / 3 / 98$ & Diesel & 36.44 & 33629.0 & 38.63 & \\
\hline 1592 & $2 / 3 / 98$ & Diesel & 41.25 & 14753.0 & 43.73 & \\
\hline 1593 & $2 / 3 / 98$ & Diesel & 40.40 & 30116.0 & 42.82 & \\
\hline 1592 & $2 / 4 / 98$ & Diesel & 19.83 & 14890.0 & 21.42 & \\
\hline 1593 & $2 / 5 / 98$ & Diesel & 34.82 & 30349.0 & 37.60 & \\
\hline 1586 & $2 / 5 / 98$ & Diesel & 25.08 & 34049.0 & 27.09 & \\
\hline 1592 & $2 / 5 / 98$ & Diesel & 33.99 & 15128.0 & 36.71 & \\
\hline 1586 & $2 / 6 / 98$ & Diesel & 77.49 & 34591.0 & 81.36 & \\
\hline 1592 & $2 / 6 / 98$ & Diesel & 61.91 & 15506.0 & 65.01 & \\
\hline 1593 & $2 / 6 / 98$ & Diesel & 29.73 & 30495.0 & 32.11 & \\
\hline 1586 & $2 / 7 / 98$ & Diesel & 0.00 & 0.0 & 0.00 & \\
\hline 1586 & $2 / 9 / 98$ & Diesel & 38.82 & 35066.0 & 40.76 & \\
\hline 1592 & $2 / 9 / 98$ & Diesel & 41.26 & 15996.0 & 43.32 & \\
\hline 1586 & $2 / 10 / 98$ & Diesel & 36.19 & 35282.0 & 38.00 & \\
\hline 1592 & $2 / 10 / 98$ & Diesel & 33.99 & 16241.0 & 35.69 & \\
\hline 1593 & $2 / 10 / 98$ & Diesel & 50.97 & 31001.0 & 53.52 & \\
\hline 1586 & $2 / 11 / 98$ & Diesel & 33.34 & 35420.0 & 35.01 & \\
\hline 1592 & $2 / 11 / 98$ & Diesel & 32.71 & 16446.0 & 34.35 & \\
\hline 1592 & $2 / 12 / 98$ & Diesel & 24.81 & 16702.0 & 26.05 & \\
\hline 1593 & $2 / 12 / 98$ & Diesel & 27.37 & 31247.0 & 28.74 & \\
\hline 1586 & $2 / 13 / 98$ & Diesel & 68.20 & 36028.0 & 71.61 & \\
\hline 1592 & $2 / 13 / 98$ & Diesel & 27.82 & 16867.0 & 29.21 & \\
\hline 1593 & $2 / 13 / 98$ & Diesel & 37.63 & 31498.0 & 39.51 & \\
\hline 1592 & $2 / 14 / 98$ & Diesel & 34.10 & 17082.0 & 35.81 & \\
\hline 1586 & $2 / 16 / 98$ & Diesel & 24.98 & 36222.0 & 26.23 & \\
\hline 1592 & $2 / 16 / 98$ & Diesel & 37.00 & 17354.0 & 38.85 & \\
\hline 1593 & $2 / 16 / 98$ & Diesel & 47.99 & 31738.0 & 50.39 & \\
\hline 1586 & $2 / 17 / 98$ & Diesel & 33.46 & 36438.0 & 33.46 & \\
\hline 1592 & $2 / 17 / 98$ & Diesel & 30.95 & 17600.0 & 30.95 & \\
\hline 1593 & $2 / 17 / 98$ & Diesel & 40.51 & 32040.0 & 40.51 & \\
\hline 1586 & $2 / 18 / 98$ & Diesel & 39.87 & 36715.0 & 39.87 & \\
\hline 1592 & $2 / 18 / 98$ & Diesel & 32.13 & 17768.0 & 32.13 & \\
\hline 1593 & $2 / 18 / 98$ & Diesel & 40.18 & 32268.0 & 40.18 & \\
\hline 1592 & $2 / 19 / 98$ & Diesel & 28.04 & 17978.0 & 28.04 & \\
\hline 1593 & $2 / 19 / 98$ & Diesel & 32.79 & 32546.0 & 32.79 & \\
\hline 1586 & $2 / 20 / 98$ & Diesel & 78.04 & 37223.0 & 78.04 & \\
\hline 1592 & $2 / 20 / 98$ & Diesel & 36.84 & 18218.0 & 36.84 & \\
\hline
\end{tabular}




\begin{tabular}{|c|c|c|c|c|c|c|}
\hline Truck & Date & Fuel & Amount & Odometer & Cost & Monthly Avg \\
\hline 1593 & $2 / 20 / 98$ & Diesel & 27.04 & 32778.0 & 27.04 & \\
\hline 1586 & $2 / 21 / 98$ & Diesel & 41.41 & 37506.0 & 41.41 & \\
\hline 1593 & $2 / 21 / 98$ & Diesel & 34.51 & 32982.0 & 34.51 & \\
\hline 1586 & $2 / 23 / 98$ & Diesel & 38.50 & 37729.0 & 38.50 & \\
\hline 1593 & $2 / 23 / 98$ & Diesel & 49.83 & 33275.0 & 49.83 & \\
\hline 1586 & $2 / 24 / 98$ & Diesel & 26.64 & 37910.0 & 26.64 & \\
\hline 1593 & $2 / 24 / 98$ & Diesel & 42.68 & 33559.0 & 42.68 & \\
\hline 1586 & $2 / 25 / 98$ & Diesel & 34.94 & 38135.0 & 34.94 & \\
\hline 1592 & $2 / 25 / 98$ & Diesel & 25.01 & 18611.0 & 25.01 & \\
\hline 1592 & $2 / 26 / 98$ & Diesel & 35.97 & 18968.0 & 35.61 & \\
\hline 1593 & $2 / 26 / 98$ & Diesel & 32.99 & 33799.0 & 32.66 & \\
\hline 1586 & $2 / 27 / 98$ & Diesel & 67.83 & 38643.0 & 67.15 & \\
\hline 1592 & $2 / 27 / 98$ & Diesel & 35.35 & 19189.0 & 35.00 & \\
\hline 1593 & $2 / 27 / 98$ & Diesel & 40.55 & 34081.0 & 40.14 & \\
\hline 1586 & $2 / 28 / 98$ & Diesel & 12.92 & 38750.0 & 12.79 & \\
\hline 1592 & $2 / 28 / 98$ & Diesel & 28.03 & 19407.0 & 27.75 & 1.03 \\
\hline 1593 & $3 / 2 / 98$ & Diesel & 40.65 & 34594.0 & 40.24 & \\
\hline 1592 & $3 / 2 / 98$ & Diesel & 32.00 & 19616.0 & 31.68 & \\
\hline 1586 & $3 / 3 / 98$ & Diesel & 41.16 & 38986.0 & 40.75 & \\
\hline 1593 & $3 / 3 / 98$ & Diesel & 37.86 & 34852.0 & 37.48 & \\
\hline 1586 & $3 / 4 / 98$ & Diesel & 36.27 & 39241.0 & 34.82 & \\
\hline 1593 & $3 / 5 / 98$ & Diesel & 51.57 & 35166.0 & 49.51 & \\
\hline 1586 & $3 / 6 / 98$ & Diesel & 75.17 & 39766.0 & 71.41 & \\
\hline 1592 & $3 / 6 / 98$ & Diesel & 42.01 & 19894.0 & 39.91 & \\
\hline 1593 & $3 / 6 / 98$ & Diesel & 37.40 & 35435.0 & 35.53 & \\
\hline 1586 & $3 / 7 / 98$ & Diesel & 14.55 & 39885.0 & 13.82 & \\
\hline 1592 & $3 / 7 / 98$ & Diesel & 28.01 & 20133.0 & 26.61 & \\
\hline 1586 & $3 / 9 / 98$ & Diesel & 37.14 & 40107.0 & 35.28 & \\
\hline 1592 & $3 / 9 / 98$ & Diesel & 31.29 & 20357.0 & 29.73 & \\
\hline 1593 & $3 / 9 / 98$ & Diesel & 40.41 & 35580.0 & 38.39 & \\
\hline 1586 & $3 / 10 / 98$ & Diesel & 29.19 & 40291.0 & 27.73 & \\
\hline 1592 & $3 / 10 / 98$ & Diesel & 29.32 & 20546.0 & 27.85 & \\
\hline 1586 & $3 / 11 / 98$ & Diesel & 32.75 & 40557.0 & 30.79 & \\
\hline 1592 & $3 / 11 / 98$ & Diesel & 33.98 & 20806.0 & 31.94 & \\
\hline 1592 & $3 / 12 / 98$ & Diesel & 28.52 & 21052.0 & 26.81 & \\
\hline 1593 & $3 / 12 / 98$ & Diesel & 21.88 & 35750.0 & 20.57 & \\
\hline $1586^{\circ}$ & $3 / 13 / 98$ & Diesel & 77.51 & 41099.0 & 72.86 & \\
\hline 1592 & $3 / 13 / 98$ & Diesel & 29.69 & 21265.0 & 27.91 & \\
\hline 1593 & $3 / 13 / 98$ & Diesel & 41.55 & 36046.0 & 39.06 & \\
\hline 1586 & $3 / 14 / 98$ & Diesel & 20.68 & 41268.0 & 19.44 & \\
\hline 1592 & $3 / 14 / 98$ & Diesel & 21.63 & 21467.0 & 20.33 & \\
\hline 1586 & $3 / 16 / 98$ & Diesel & 31.87 & 41489.0 & 29.96 & \\
\hline 1592 & $3 / 16 / 98$ & Diesel & 36.69 & 21673.0 & 34.49 & \\
\hline 1593 & $3 / 16 / 98$ & Diesel & 38.01 & 36711.0 & 35.73 & \\
\hline 1592 & $3 / 17 / 98$ & Diesel & 31.53 & 21901.0 & 29.64 & \\
\hline 1593 & $3 / 17 / 98$ & Diesel & 42.95 & 36994.0 & 40.37 & \\
\hline 1586 & $3 / 17 / 98$ & Diesel & 32.13 & 41605.0 & 30.20 & \\
\hline 1586 & $3 / 18 / 98$ & Diesel & 35.84 & 41971.0 & 33.69 & \\
\hline 1592 & $3 / 18 / 98$ & Diesel & 30.22 & 22111.0 & 28.41 & \\
\hline 1593 & $3 / 18 / 98$ & Diesel & 24.02 & 37195.0 & 22.58 & \\
\hline 1592 & $3 / 19 / 98$ & Diesel & 19.96 & 22350.0 & 19.16 & \\
\hline 1593 & $3 / 19 / 98$ & Diesel & 33.86 & 37478.0 & 32.51 & \\
\hline 1586 & $3 / 20 / 98$ & Diesel & 66.23 & 42481.0 & 63.58 & \\
\hline 1592 & $3 / 20 / 98$ & Diesel & 33.85 & 22532.0 & 32.50 & \\
\hline
\end{tabular}




\begin{tabular}{|c|c|c|c|c|c|c|}
\hline Truck & Date & Fuel & Amount & Odometer & Cost & Monthly Avg \\
\hline 1593 & $3 / 20 / 98$ & Diesel & 42.62 & 37758.0 & 40.92 & \\
\hline 1592 & $3 / 21 / 98$ & Diesel & 27.26 & 22754.0 & 26.17 & \\
\hline 1586 & $3 / 23 / 98$ & Diesel & 50.64 & 42844.0 & 49.63 & \\
\hline 1592 & $3 / 23 / 98$ & Diesel & 38.05 & 23004.0 & 37.29 & \\
\hline 1593 & $3 / 23 / 98$ & Diesel & 43.62 & 38003.0 & 42.75 & \\
\hline 1592 & $3 / 24 / 98$ & Diesel & 28.59 & 23204.0 & 28.02 & \\
\hline 1593 & $3 / 24 / 98$ & Diesel & 42.84 & 38281.0 & 41.98 & \\
\hline 1592 & $3 / 25 / 98$ & Diesel & 31.85 & 23427.0 & 31.85 & \\
\hline \begin{tabular}{|l|}
1586 \\
\end{tabular} & $3 / 27 / 98$ & Diesel & 72.56 & 43367.0 & 79.82 & \\
\hline 1592 & $3 / 27 / 98$ & Diesel & 31.44 & 23991.0 & 34.58 & \\
\hline 1593 & $3 / 27 / 98$ & Diesel & 39.80 & 38531.0 & 43.78 & \\
\hline 1586 & $3 / 28 / 98$ & Diesel & 33.19 & 43589.0 & 36.51 & \\
\hline 1592 & $3 / 28 / 98$ & Diesel & 40.78 & 24256.0 & 44.86 & \\
\hline 1593 & $3 / 28 / 98$ & Diesel & 20.52 & 38895.0 & 22.57 & \\
\hline 1586 & $3 / 30 / 98$ & Diesel & 27.01 & 43801.0 & 29.71 & \\
\hline 1592 & $3 / 30 / 98$ & Diesel & 30.54 & 24502.0 & 33.59 & \\
\hline 1593 & $3 / 30 / 98$ & Diesel & 44.57 & 39225.0 & 49.03 & \\
\hline 1586 & $3 / 31 / 98$ & Diesel & 33.44 & 44022.0 & 36.78 & \\
\hline 1592 & $3 / 31 / 98$ & Diesel & 37.54 & 24740.0 & 41.29 & \\
\hline 1593 & $3 / 31 / 98$ & Diesel & 43.87 & 39545.0 & 48.26 & 0.99 \\
\hline 1586 & $4 / 1 / 98$ & Diesel & 33.92 & 44256.0 & 37.31 & \\
\hline 1592 & $4 / 1 / 98$ & Diese! & 24.16 & 24944.0 & 26.58 & \\
\hline 1592 & $4 / 2 / 98$ & Diesel & 35.12 & 25183.0 & 38.63 & \\
\hline 1593 & $4 / 2 / 98$ & Diesel & 46.38 & 39878.0 & 51.02 & \\
\hline 1586 & $4 / 3 / 98$ & Diesel & 71.42 & 44766.0 & 76.48 & \\
\hline 1592 & $4 / 3 / 98$ & Diesel & 31.85 & 25385.0 & 34.09 & \\
\hline 1593 & $4 / 3 / 98$ & Diesel & 43.08 & 40203.0 & 46.10 & \\
\hline 1592 & $4 / 4 / 98$ & Diesel & 28.81 & 25592.0 & 30.83 & \\
\hline 1586 & $4 / 6 / 98$ & Diesel & 53.83 & 45043.0 & 57.60 & \\
\hline 1592 & $4 / 6 / 98$ & Diesel & 39.68 & 25859.0 & 42.46 & \\
\hline 1593 & $4 / 6 / 98$ & Diesel & 50.24 & 40538.0 & 53.76 & \\
\hline 1586 & $4 / 7 / 98$ & Diesel & 45.88 & 45357.0 & 49.09 & \\
\hline 1592 & $4 / 7 / 98$ & Diesel & 30.59 & 26065.0 & 32.73 & \\
\hline 1593 & $4 / 7 / 98$ & Diesel & 50.91 & 40916.0 & 54.47 & \\
\hline 1592 & $4 / 8 / 98$ & Diesel & 36.82 & 26306.0 & 38.66 & \\
\hline 1593 & $4 / 8 / 98$ & Diesel & 27.08 & 41142.0 & 28.43 & \\
\hline 1586 & $4 / 8 / 98$ & Diesel & 35.10 & 45626.0 & 36.86 & \\
\hline 1586 & $4 / 9 / 98$ & Diesel & 29.87 & 45840.0 & 31.36 & \\
\hline 1592 & $4 / 9 / 98$ & Diesel & 23.68 & 26590.0 & 24.86 & \\
\hline 1593 & $4 / 9 / 98$ & Diesel & 36.51 & 41375.0 & 38.34 & \\
\hline 1586 & $4 / 10 / 98$ & Diesel & 38.73 & 46112.0 & 40.28 & \\
\hline 1592 & $4 / 10 / 98$ & Diesel & 31.68 & 26729.0 & 32.95 & \\
\hline 1593 & $4 / 10 / 98$ & Diesel & 46.76 & 41707.0 & 48.63 & \\
\hline 1586 & $4 / 11 / 98$ & Diesel & 34.80 & 46425.0 & 36.19 & \\
\hline 1586 & $4 / 11 / 98$ & Diesel & 55.79 & 46761.0 & 58.02 & \\
\hline 1592 & $4 / 11 / 98$ & Diesel & 36.35 & 26956.0 & 37.80 & \\
\hline 1593 & $4 / 11 / 98$ & Diesel & 24.36 & 41903.0 & 25.33 & \\
\hline 1586 & $4 / 13 / 98$ & Diesel & 32.70 & 46981.0 & 34.01 & \\
\hline 1592 & $4 / 13 / 98$ & Diesel & 40.77 & 27213.0 & 42.40 & \\
\hline 1593 & $4 / 13 / 98$ & Diesel & 39.03 & 42150.0 & 40.59 & \\
\hline 1586 & $4 / 14 / 98$ & Diesel & 32.50 & 47210.0 & 33.80 & \\
\hline 1592 & $4 / 14 / 98$ & Diesel & 30.28 & 27434.0 & 31.49 & \\
\hline 1593 & $4 / 14 / 98$ & Diesel & 36.99 & 42434.0 & 38.47 & \\
\hline 1586 & $4 / 15 / 98$ & Diesel & 39.77 & 47495.0 & 40.96 & \\
\hline
\end{tabular}




\begin{tabular}{|c|c|c|c|c|c|c|}
\hline Truck & Date & Fuel & Amount & Odometer & Cost & Monthly Avg \\
\hline 1592 & $4 / 15 / 98$ & Diesel & 31.02 & 27688.0 & 31.95 & \\
\hline 1593 & $4 / 15 / 98$ & Diesel & 39.47 & 42709.0 & 40.65 & \\
\hline 1586 & $4 / 16 / 98$ & Diesel & 32.35 & 47706.0 & 33.32 & \\
\hline 1592 & $4 / 16 / 98$ & Diesel & 27.29 & 27925.0 & 28.11 & \\
\hline 1586 & $4 / 17 / 98$ & Diesel & 60.84 & 48218.0 & 63.27 & \\
\hline 1592 & $4 / 17 / 98$ & Diesel & 32.05 & 28057.0 & 33.33 & \\
\hline 1593 & $4 / 17 / 98$ & Diesel & 44.30 & 43038.0 & 46.07 & \\
\hline 1586 & $4 / 18 / 98$ & Diesel & 36.99 & 48497.0 & 38.47 & \\
\hline 1592 & $4 / 18 / 98$ & Diesel & 40.93 & 28372.0 & 42.57 & \\
\hline 1593 & $4 / 18 / 98$ & Diesel & 27.15 & 43300.0 & 28.24 & \\
\hline 1586 & $4 / 20 / 98$ & Diesel & 39.38 & 48770.0 & 40.96 & \\
\hline 1592 & $4 / 20 / 98$ & Diesel & 31.17 & 28600.0 & 32.42 & \\
\hline 1593 & $4 / 20 / 98$ & Diesel & 43.80 & 43539.0 & 45.55 & \\
\hline 1586 & $4 / 21 / 98$ & Diesel & 32.25 & 49001.0 & 33.86 & \\
\hline 1592 & $4 / 21 / 98$ & Diesel & 32.67 & 28806.0 & 34.30 & \\
\hline 1593 & $4 / 21 / 98$ & Diesel & 0.00 & 0.0 & 0.00 & \\
\hline 1586 & $4 / 22 / 98$ & Diesel & 36.36 & 49256.0 & 38.18 & \\
\hline 1592 & $4 / 22 / 98$ & Diesel & 16.31 & 29015.0 & 17.13 & \\
\hline 1593 & $4 / 22 / 98$ & Diesel & 40.78 & 44091.0 & 42.82 & \\
\hline 1586 & $4 / 23 / 98$ & Diesel & 36.07 & 49504.0 & 37.87 & \\
\hline 1592 & $4 / 23 / 98$ & Diesel & 33.98 & 29269.0 & 35.68 & \\
\hline 1593 & $4 / 23 / 98$ & Diesel & 52.07 & 44477.0 & 54.67 & \\
\hline 1586 & $4 / 24 / 98$ & Diesel & 68.18 & 50015.0 & 72.27 & \\
\hline 1592 & $4 / 24 / 98$ & Diesel & 31.86 & 29458.0 & 33.77 & \\
\hline 1593 & $4 / 24 / 98$ & Diesel & 37.16 & 44777.0 & 39.39 & \\
\hline 1586 & $4 / 25 / 98$ & Diesel & 24.30 & 50211.0 & 25.70 & \\
\hline 1592 & $4 / 25 / 98$ & Diesel & 26.37 & 29619.0 & 27.98 & \\
\hline 1592 & $4 / 25 / 98$ & Diesel & 53.25 & 29935.0 & 56.45 & \\
\hline 1593 & $4 / 25 / 98$ & Diesel & 37.72 & 45001.0 & 39.98 & \\
\hline 1586 & $4 / 27 / 98$ & Diesel & 28.69 & 50433.0 & 30.41 & \\
\hline 1592 & $4 / 27 / 98$ & Diesel & 30.12 & 30209.0 & 31.93 & \\
\hline 1593 & $4 / 27 / 98$ & Diesel & 36.81 & 45307.0 & 39.02 & \\
\hline 1586 & $4 / 28 / 98$ & Diesel & 45.08 & 50722.0 & 48.69 & \\
\hline 1592 & $4 / 28 / 98$ & Diesel & 21.35 & 30410.0 & 23.06 & \\
\hline 1593 & $4 / 28 / 98$ & Diesel & 32.22 & 45583.0 & 34.82 & \\
\hline 1586 & $4 / 29 / 98$ & Diesel & 30.07 & 50958.0 & 33.08 & \\
\hline 1592 & $4 / 29 / 98$ & Diesel & 35.85 & 30619.0 & 39.44 & \\
\hline 1593 & $4 / 29 / 98$ & Diesel & 48.13 & 45892.0 & 52.97 & \\
\hline 1586 & $4 / 30 / 98$ & Diesel & 26.44 & 51206.0 & 29.08 & \\
\hline 1592 & $4 / 30 / 98$ & Diesel & 33.80 & 30825.0 & 37.18 & \\
\hline 1593 & $4 / 30 / 98$ & Diesel & 53.84 & 46283.0 & 59.22 & 1.06 \\
\hline 1592 & $5 / 1 / 98$ & Diesel & 31.19 & 31026.0 & 34.31 & \\
\hline 1586 & $5 / 1 / 98$ & Diesel & 81.04 & 51780.0 & 89.14 & \\
\hline 1593 & $5 / 1 / 98$ & Diesel & 41.12 & 46619.0 & 45.23 & \\
\hline 1586 & $5 / 2 / 98$ & Diesel & 34.76 & 51987.0 & 38.24 & \\
\hline 1592 & $5 / 2 / 98$ & Diesel & 31.39 & 31265.0 & 34.53 & \\
\hline 1593 & $5 / 2 / 98$ & Diesel & 47.04 & 46925.0 & 51.74 & \\
\hline 1586 & $5 / 4 / 98$ & Diesel & 48.29 & 52412.0 & 53.12 & \\
\hline 1592 & $5 / 4 / 98$ & Diesel & 33.08 & 31519.0 & 36.39 & \\
\hline 1593 & $5 / 4 / 98$ & Diesel & 48.63 & 47222.0 & 53.49 & \\
\hline 1586 & $5 / 5 / 98$ & Diesel & 57.19 & 52669.0 & 62.91 & \\
\hline 1592 & $5 / 5 / 98$ & Diesel & 37.99 & 31765.0 & 41.79 & \\
\hline 1593 & $5 / 5 / 98$ & Diesel & 45.28 & 47575.0 & 49.81 & \\
\hline 1592 & $5 / 6 / 98$ & Diesel & 23.71 & 31970.0 & 26.08 & \\
\hline
\end{tabular}




\begin{tabular}{|c|c|c|c|c|c|c|}
\hline Truck & Date & Fuel & Amount & Odometer & Cost & Monthly Avg \\
\hline 1593 & $5 / 6 / 98$ & Diesel & 39.63 & 47832.0 & 43.59 & \\
\hline 1586 & $5 / 6 / 98$ & Diesel & 45.19 & 53060.0 & 49.71 & \\
\hline 1586 & $5 / 7 / 98$ & Diesel & 59.08 & 53523.0 & 64.99 & \\
\hline 1593 & $5 / 7 / 98$ & Diesel & 36.33 & 48107.0 & 39.96 & \\
\hline 1592 & $5 / 7 / 98$ & Diesel & 20.89 & 32173.0 & 22.98 & \\
\hline 1592 & $5 / 8 / 98$ & Diesel & 37.14 & 32430.0 & 40.85 & \\
\hline 1593 & $5 / 8 / 98$ & Diesel & 42.67 & 48430.0 & 46.90 & \\
\hline 1593 & $5 / 9 / 98$ & Diesel & 40.03 & 487.38 .0 & 44.03 & \\
\hline 1592 & $5 / 9 / 98$ & Diesel & 0.00 & 0.0 & 0.00 & \\
\hline 1586 & $5 / 10 / 98$ & Diesel & 78.19 & 54090.0 & 86.01 & \\
\hline 1586 & $5 / 11 / 98$ & Diesel & 36.03 & 54395.0 & 39.63 & \\
\hline 1592 & $5 / 11 / 98$ & Diesel & 33.00 & 32852.0 & 36.30 & \\
\hline 1593 & $5 / 11 / 98$ & Diesel & 43.00 & 48980.0 & 47.30 & \\
\hline 1586 & $5 / 12 / 98$ & Diesel & 62.01 & 55035.0 & 65.73 & \\
\hline 1592 & $5 / 12 / 98$ & Diesel & 24.22 & 33082.0 & 25.67 & \\
\hline 1593 & $5 / 12 / 98$ & Diesel & 11.00 & 49065.0 & 11.66 & \\
\hline 1586 & $5 / 13 / 98$ & Diesel & 44.59 & 55392.0 & 46.82 & \\
\hline 1592 & $5 / 13 / 98$ & Diesel & 46.01 & 33323.0 & 48.31 & \\
\hline 1593 & $5 / 13 / 98$ & Diesel & 36.01 & 49357.0 & 37.81 & \\
\hline 1586 & $5 / 14 / 98$ & Diesel & 56.63 & 55867.0 & 59.46 & \\
\hline 1592 & $5 / 14 / 98$ & Diesel & 27.40 & 33531.0 & 28.77 & \\
\hline 1593 & $5 / 14 / 98$ & Diesel & 44.81 & 49551.0 & 47.05 & \\
\hline 1586 & $5 / 15 / 98$ & Diesel & 44.84 & 56155.0 & 45.28 & \\
\hline 1592 & $5 / 15 / 98$ & Diesel & 29.85 & 33761.0 & 30.15 & \\
\hline 1593 & $5 / 15 / 98$ & Diesel & 33.34 & 49808.0 & 33.67 & \\
\hline 1593 & $5 / 15 / 98$ & Diesel & 17.38 & 49748.0 & 18.25 & \\
\hline 1586 & $5 / 16 / 98$ & Diesel & 29.66 & 56380.0 & 29.96 & \\
\hline 1592 & $5 / 16 / 98$ & Diesel & 32.01 & 33983.0 & 32.33 & \\
\hline 1593 & $5 / 16 / 98$ & Diesel & 28.68 & 50252.0 & 28.97 & \\
\hline 1586 & $5 / 18 / 98$ & Diesel & 44.01 & 56652.0 & 44.45 & \\
\hline 1592 & $5 / 18 / 98$ & Diesel & 33.00 & 34202.0 & 33.33 & \\
\hline 1593 & $5 / 18 / 98$ & Diesel & 38.16 & 50492.0 & 38.54 & \\
\hline 1592 & $5 / 19 / 98$ & Diesel & 42.99 & 34532.0 & 43.42 & \\
\hline 1593 & $5 / 19 / 98$ & Diesel & 29.81 & 50717.0 & 30.11 & \\
\hline 1586 & $5 / 19 / 98$ & Diesel & 58.98 & 57100.0 & 59.57 & \\
\hline 1586 & $5 / 20 / 98$ & Diesel & 36.99 & 57388.0 & 36.62 & \\
\hline 1593 & $5 / 20 / 98$ & Diesel & 47.01 & 51010.0 & 46.54 & \\
\hline 1586 & $5 / 21 / 98$ & Diesel & 52.78 & 57635.0 & 51.72 & \\
\hline 1592 & $5 / 21 / 98$ & Diesel & 53.07 & 34881.0 & 52.01 & \\
\hline 1593 & $5 / 21 / 98$ & Diesel & 41.57 & 51295.0 & 40.74 & \\
\hline 1586 & $5 / 22 / 98$ & Diesel & 40.75 & 58117.0 & 39.94 & \\
\hline 1592 & $5 / 22 / 98$ & Diesel & 57.95 & 35300.0 & 56.79 & \\
\hline 1593 & $5 / 22 / 98$ & Diesel & 37.37 & 51582.0 & 36.62 & \\
\hline 1586 & $5 / 23 / 98$ & Diesel & 60.63 & 58570.0 & 59.42 & \\
\hline 1592 & $5 / 23 / 98$ & Diesel & 30.87 & 35568.0 & 30.25 & \\
\hline 1593 & $5 / 23 / 98$ & Diesel & 33.19 & 51853.0 & 32.53 & \\
\hline 1592 & $5 / 25 / 98$ & Diesel & 44.00 & 35851.0 & 43.12 & \\
\hline 1593 & $5 / 25 / 98$ & Diesel & 44.01 & 52151.0 & 43.13 & \\
\hline 1586 & $5 / 25 / 98$ & Diesel & 35.00 & 58836.0 & 34.30 & \\
\hline 1586 & $5 / 26 / 98$ & Diesel & 17.25 & 58911.0 & 16.91 & \\
\hline 1586 & $5 / 26 / 98$ & Diesel & 61.00 & 59421.0 & 59.17 & \\
\hline 1593 & $5 / 26 / 98$ & Diesel & 29.02 & 52326.0 & 28.15 & \\
\hline 1586 & $5 / 27 / 98$ & Diesel & 42.97 & 59769.0 & 41.68 & \\
\hline 1593 & $5 / 27 / 98$ & Diesel & 39.00 & 52612.0 & 37.83 & \\
\hline
\end{tabular}




\begin{tabular}{|c|c|c|c|c|c|c|}
\hline Truck & Date & Fuel & Amount & Odometer & Cost & Monthly Avg \\
\hline 1586 & $5 / 28 / 98$ & Diesel & 27.28 & 59999.0 & 26.46 & \\
\hline 1592 & $5 / 28 / 98$ & Diesel & 58.09 & 36276.0 & 56.35 & \\
\hline 1593 & $5 / 28 / 98$ & Diesel & 33.69 & 52754.0 & 32.68 & \\
\hline 1586 & $5 / 29 / 98$ & Diesel & 45.00 & 60280.0 & 43.65 & \\
\hline 1592 & $5 / 29 / 98$ & Diesel & 28.30 & 36490.0 & 27.45 & \\
\hline 1593 & $5 / 29 / 98$ & Diesel & 43.00 & 53178.0 & 41.71 & \\
\hline 1586 & $5 / 30 / 98$ & Diesel & 55.94 & 60634.0 & 54.26 & \\
\hline 1592 & $5 / 30 / 98$ & Diesel & 11.52 & 36528.0 & 11.17 & \\
\hline 1593 & $5 / 30 / 98$ & Diesel & 55.46 & 53692.0 & 53.80 & \\
\hline 1592 & $5 / 30 / 98$ & Diesel & 15.98 & 36650.0 & 15.50 & 1.03 \\
\hline 1586 & $6 / 1 / 98$ & Diesel & 66.00 & 61170.0 & 64.02 & \\
\hline 1592 & $6 / 1 / 98$ & Diesel & 47.00 & 37002.0 & 45.59 & \\
\hline 1593 & $6 / 1 / 98$ & Diesel & 54.99 & 53969.0 & 53.34 & \\
\hline 1592 & $6 / 2 / 98$ & Diesel & 32.00 & 37207.0 & 30.08 & \\
\hline 1593 & $6 / 2 / 98$ & Diesel & 37.99 & 54245.0 & 35.71 & \\
\hline 1586 & $6 / 3 / 98$ & Diesel & 43.00 & 61484.0 & 40.42 & \\
\hline 1592 & $6 / 3 / 98$ & Diesel & 38.00 & 37466.0 & 35.72 & \\
\hline 1593 & $6 / 3 / 98$ & Diesel & 37.99 & 54512.0 & 35.71 & \\
\hline 1586 & $6 / 5 / 98$ & Diesel & 44.02 & 62298.0 & 41.38 & \\
\hline 1593 & $6 / 5 / 98$ & Diesel & 39.79 & 55073.0 & 37.40 & \\
\hline 1593 & $6 / 5 / 98$ & Diesel & 10.03 & 55114.0 & 9.43 & \\
\hline 1586 & $6 / 6 / 98$ & Diesel & 45.05 & 62545.0 & 42.35 & \\
\hline 1592 & $6 / 6 / 98$ & Diesel & 84.02 & 37708.0 & 78.98 & \\
\hline 1593 & $6 / 6 / 98$ & Diesel & 50.00 & 55469.0 & 47.59 & \\
\hline 1586 & $6 / 8 / 98$ & Diesel & 39.01 & 62812.0 & 37.84 & \\
\hline 1593 & $6 / 8 / 98$ & Diesel & 34.42 & 55739.0 & 33.39 & \\
\hline 1586 & $6 / 9 / 98$ & Diesel & 58.02 & 63254.0 & 56.86 & \\
\hline 1592 & $6 / 9 / 98$ & Diesel & 67.01 & 38669.0 & 65.67 & \\
\hline 1593 & $6 / 9 / 98$ & Diesel & 48.00 & 56048.0 & 47.04 & \\
\hline 1592 & $6 / 10 / 98$ & Diesel & 24.00 & 38879.0 & 23.52 & \\
\hline 1593 & $6 / 10 / 98$ & Diesel & 37.99 & 56332.0 & 37.23 & \\
\hline 1586 & $6 / 11 / 98$ & Diesel & 58.76 & 63715.0 & 57.58 & \\
\hline 1592 & $6 / 11 / 98$ & Diesel & 39.08 & 39116.0 & 38.30 & \\
\hline 1593 & $6 / 11 / 98$ & Diesel & 35.84 & 56633.0 & 35.12 & \\
\hline 1592 & $6 / 12 / 98$ & Diesel & 26.40 & 39325.0 & 26.14 & \\
\hline 1593 & $6 / 12 / 98$ & Diesel & 31.16 & 56909.0 & 30.85 & \\
\hline 1586 & $6 / 13 / 98$ & Diesel & 55.00 & 64604.0 & 54.45 & \\
\hline 1592 & $6 / 13 / 98$ & Diesel & 30.71 & 39519.0 & 30.40 & \\
\hline 1593 & $6 / 13 / 98$ & Diesel & 52.86 & 57178.0 & 52.33 & \\
\hline 1586 & $6 / 15 / 98$ & Diesel & 32.00 & 64852.0 & 31.68 & \\
\hline 1592 & $6 / 15 / 98$ & Diesel & 40.02 & 39787.0 & 39.62 & \\
\hline 1593 & $6 / 15 / 98$ & Diesel & 48.01 & 57487.0 & 47.53 & \\
\hline 1586 & $6 / 16 / 98$ & Diesel & 62.01 & 65291.0 & 61.39 & \\
\hline 1592 & $6 / 16 / 98$ & Diesel & 37.00 & 40019.0 & 36.63 & \\
\hline 1593 & $6 / 16 / 98$ & Diesel & 34.99 & 57700.0 & 34.64 & \\
\hline 1586 & $6 / 17 / 98$ & Diesel & 39.01 & 65596.0 & 39.40 & \\
\hline 1592 & $6 / 17 / 98$ & Diesel & 33.99 & 40257.0 & 34.33 & \\
\hline 1593 & $6 / 17 / 98$ & Diesel & 43.01 & 58008.0 & 43.44 & \\
\hline 1586 & $6 / 18 / 98$ & Diesel & 57.96 & 66046.0 & 58.54 & \\
\hline 1592 & $6 / 18 / 98$ & Diesel & 22.91 & 40479.0 & 23.14 & \\
\hline 1593 & $6 / 18 / 98$ & Diesel & 39.44 & 58547.0 & 39.83 & \\
\hline 1593 & $6 / 18 / 98$ & Diesel & 29.94 & 58218.0 & 30.24 & \\
\hline 1586 & $6 / 19 / 98$ & Diesel & 44.88 & 66389.0 & 45.33 & \\
\hline 1592 & $6 / 19 / 98$ & Diesel & 36.71 & 40699.0 & 37.08 & \\
\hline
\end{tabular}




\begin{tabular}{|c|c|c|c|c|c|c|}
\hline Truck & Date & Fuel & Amount & Odometer & Cost & Monthly Avg \\
\hline 1593 & $6 / 19 / 98$ & Diesel & 48.79 & 58973.0 & 49.28 & \\
\hline 1586 & $6 / 20 / 98$ & Diesel & 56.98 & 66832.0 & 57.55 & \\
\hline 1592 & $6 / 20 / 98$ & Diesel & 21.91 & 40900.0 & 22.13 & \\
\hline 1592 & $6 / 20 / 98$ & Diesel & 60.07 & 41282.0 & 60.67 & \\
\hline 1593 & $6 / 20 / 98$ & Diesel & 44.80 & 58986.0 & 45.25 & \\
\hline 1586 & $6 / 22 / 98$ & Diesel & 34.00 & 67096.0 & 34.34 & \\
\hline 1592 & $6 / 22 / 98$ & Diesel & 39.97 & 41574.0 & 40.37 & \\
\hline 1593 & $6 / 22 / 98$ & Diesel & 46.99 & 59700.0 & 47.46 & \\
\hline 1586 & $6 / 23 / 98$ & Diesel & 56.01 & 67535.0 & 56.01 & \\
\hline 1593 & $6 / 23 / 98$ & Diesel & 43.00 & 60000.0 & 43.00 & \\
\hline 1586 & $6 / 24 / 98$ & Diesel & 48.01 & 67880.0 & 48.01 & \\
\hline 1592 & $6 / 24 / 98$ & Diesel & 61.01 & 42019.0 & 61.01 & \\
\hline 1593 & $6 / 24 / 98$ & Diesel & 38.01 & 60275.0 & 38.01 & \\
\hline 1586 & $6 / 25 / 98$ & Diesel & 60.81 & 68343.0 & 60.81 & \\
\hline 1592 & $6 / 25 / 98$ & Diesel & 28.52 & 42236.0 & 28.52 & \\
\hline 1593 & $6 / 25 / 98$ & Diesel & 37.28 & 60557.0 & 37.28 & \\
\hline 1586 & $6 / 26 / 98$ & Diesel & 45.05 & 68653.0 & 44.60 & \\
\hline 1592 & $6 / 26 / 98$ & Diesel & 34.35 & 42452.0 & 34.01 & \\
\hline 1593 & $6 / 26 / 98$ & Diesel & 40.12 & 60838.0 & 39.72 & \\
\hline 1586 & $6 / 27 / 98$ & Diesel & 45.48 & 68969.0 & 45.03 & \\
\hline 1592 & $6 / 27 / 98$ & Diesel & 39.47 & 42710.0 & 39.08 & \\
\hline 1593 & $6 / 27 / 98$ & Diesel & 25.01 & 61033.0 & 24.76 & \\
\hline 1586 & $6 / 29 / 98$ & Diesel & 36.00 & 69222.0 & 35.64 & \\
\hline 1592 & $6 / 29 / 98$ & Diesel & 36.98 & 42965.0 & 36.61 & \\
\hline 1593 & $6 / 29 / 98$ & Diesel & 44.02 & 61303.0 & 43.58 & \\
\hline 1593 & $6 / 30 / 98$ & Diesel & 23.30 & 61481.0 & 23.07 & \\
\hline 1592 & $6 / 30 / 98$ & Diesel & 31.05 & 43171.0 & 30.74 & \\
\hline 1586 & $6 / 30 / 98$ & Diesel & 54.02 & 69660.0 & 53.48 & 0.99 \\
\hline 1586 & $7 / 1 / 98$ & Diesel & 42.00 & 69974.0 & 41.58 & \\
\hline 1592 & $7 / 1 / 98$ & Diesel & 27.99 & 43377.0 & 27.71 & \\
\hline 1593 & $7 / 1 / 98$ & Diesel & 39.01 & 61759.0 & 38.62 & \\
\hline 1586 & $7 / 2 / 98$ & Diesel & 56.56 & 70417.0 & 55.43 & \\
\hline 1592 & $7 / 2 / 98$ & Diesel & 36.97 & 43635.0 & 36.23 & \\
\hline 1593 & $7 / 2 / 98$ & Diesel & 52.24 & 62106.0 & 51.20 & \\
\hline 1586 & $7 / 3 / 98$ & Diesel & 17.52 & 70636.0 & 17.17 & \\
\hline 1593 & $7 / 3 / 98$ & Diesel & 31.42 & 62382.0 & 30.79 & \\
\hline 1592 & $7 / 3 / 98$ & Diesel & 21.14 & 43846.0 & 20.72 & \\
\hline 1586 & $7 / 4 / 98$ & Diesel & 71.74 & 71404.0 & 70.31 & \\
\hline 1592 & $7 / 4 / 98$ & Diesel & 51.02 & 44103.0 & 50.00 & \\
\hline 1593 & $7 / 4 / 98$ & Diesel & 51.39 & 62600.0 & 50.36 & \\
\hline 1586 & $7 / 6 / 98$ & Diesel & 18.55 & 71250.0 & 18.18 & \\
\hline 1586 & $7 / 6 / 98$ & Diesel & 52.99 & 71576.0 & 51.93 & \\
\hline 1592 & $7 / 6 / 98$ & Diesel & 27.75 & 44342.0 & 27.20 & \\
\hline 1593 & $7 / 6 / 98$ & Diesel & 37.87 & 63275.0 & 37.11 & \\
\hline 1586 & $7 / 7 / 98$ & Diesel & 60.01 & 72025.0 & 58.81 & \\
\hline 1592 & $7 / 7 / 98$ & Diesel & 41.04 & 44521.0 & 40.22 & \\
\hline 1586 & $7 / 8 / 98$ & Diesel & 44.99 & 72346.0 & 43.64 & \\
\hline 1592 & $7 / 8 / 98$ & Diesel & 20.54 & 44722.0 & 19.92 & \\
\hline 1593 & $7 / 8 / 98$ & Diesel & 31.92 & 63543.0 & 30.96 & \\
\hline 1586 & $7 / 9 / 98$ & Diesel & 59.75 & 72811.0 & 57.96 & \\
\hline 1592 & $7 / 9 / 98$ & Diesel & 38.83 & 45170.0 & 37.67 & \\
\hline 1593 & $7 / 9 / 98$ & Diesel & 40.44 & 63825.0 & 39.23 & \\
\hline 1592 & $7 / 9 / 98$ & Diesel & 39.19 & 44946.0 & 38.01 & \\
\hline 1586 & $7 / 10 / 98$ & Diesel & 44.82 & 73120.0 & 43.48 & \\
\hline
\end{tabular}




\begin{tabular}{|c|c|c|c|c|c|c|}
\hline Truck & Date & Fuel & Amount & Odometer & Cost & Monthly Avg \\
\hline 1592 & $7 / 10 / 98$ & Diesel & 29.02 & 45377.0 & 28.15 & \\
\hline 1593 & $7 / 10 / 98$ & Diesel & 41.58 & 64097.0 & 40.33 & \\
\hline 1586 & $7 / 11 / 98$ & Diesel & 36.58 & 73416.0 & 35.48 & \\
\hline 1592 & $7 / 11 / 98$ & Diesel & 32.19 & 45677.0 & 31.22 & \\
\hline 1593 & $7 / 11 / 98$ & Diesel & 19.46 & 64497.0 & 18.88 & \\
\hline 1586 & $7 / 13 / 98$ & Diesel & 48.02 & 73742.0 & 46.58 & \\
\hline 1592 & $7 / 13 / 98$ & Diesel & 41.99 & 45900.0 & 40.73 & \\
\hline 1593 & $7 / 13 / 98$ & Diesel & 36.00 & 64859.0 & 34.92 & \\
\hline 1586 & $7 / 14 / 98$ & Diesel & 59.99 & 74190.0 & 58.19 & \\
\hline 1592 & $7 / 14 / 98$ & Diesel & 24.42 & 46108.0 & 23.69 & \\
\hline 1593 & $7 / 14 / 98$ & Diesel & 12.29 & 64999.0 & 11.92 & \\
\hline 1593 & $7 / 14 / 98$ & Diesel & 39.00 & 65226.0 & 37.83 & \\
\hline 1593 & $7 / 15 / 98$ & Diesel & 36.33 & 65518.0 & 37.42 & \\
\hline 1592 & $7 / 15 / 98$ & Diesel & 33.81 & 46345.0 & 34.82 & \\
\hline 1586 & $7 / 16 / 98$ & Diesel & 57.57 & 74554.0 & 59.87 & \\
\hline 1592 & $7 / 16 / 98$ & Diesel & 30.25 & 46544.0 & 31.46 & \\
\hline 1593 & $7 / 16 / 98$ & Diesel & 44.89 & 65789.0 & 46.69 & \\
\hline 1586 & $7 / 17 / 98$ & Diesel & 48.11 & 75301.0 & 50.03 & \\
\hline 1592 & $7 / 17 / 98$ & Diesel & 31.78 & 46788.0 & 33.05 & \\
\hline 1593 & $7 / 17 / 98$ & Diesel & 27.76 & 66016.0 & 28.87 & \\
\hline 1586 & $7 / 18 / 98$ & Diesel & 34.99 & 75578.0 & 36.39 & \\
\hline 1592 & $7 / 18 / 98$ & Diesel & 33.94 & 47001.0 & 35.30 & \\
\hline 1593 & $7 / 18 / 98$ & Diesel & 40.94 & 66316.0 & 42.58 & \\
\hline 1586 & $7 / 20 / 98$ & Diesel & 45.01 & 75891.0 & 46.81 & \\
\hline 1592 & $7 / 20 / 98$ & Diesel & 38.01 & 47271.0 & 39.53 & \\
\hline 1593 & $7 / 20 / 98$ & Diesel & 37.02 & 66535.0 & 38.50 & \\
\hline 1592 & $7 / 21 / 98$ & Diesel & 30.02 & 47475.0 & 31.22 & \\
\hline 1593 & $7 / 21 / 98$ & Diesel & 42.01 & 66813.0 & 43.69 & \\
\hline 1592 & $7 / 22 / 98$ & Diesel & 24.58 & 47686.0 & 23.60 & \\
\hline 1593 & $7 / 22 / 98$ & Diesel & 41.01 & 67100.0 & 39.37 & \\
\hline 1586 & $7 / 22 / 98$ & Diesel & 47.00 & 76206.0 & 45.12 & \\
\hline 1586 & $7 / 23 / 98$ & Diesel & 67.59 & 76682.0 & 64.89 & \\
\hline 1592 & $7 / 23 / 98$ & Diese! & 29.80 & 47887.0 & 28.61 & \\
\hline 1593 & $7 / 23 / 98$ & Diesel & 40.51 & 67388.0 & 38.89 & \\
\hline 1586 & $7 / 24 / 98$ & Diesel & 77.92 & 77230.0 & 74.80 & \\
\hline 1592 & $7 / 24 / 98$ & Diesel & 40.87 & 48165.0 & 39.24 & \\
\hline 1586 & $7 / 25 / 98$ & Diesel & 54.96 & 77672.0 & 52.76 & \\
\hline 1592 & $7 / 25 / 98$ & Diesel & 27.10 & 48356.0 & 26.02 & \\
\hline 1593 & $7 / 25 / 98$ & Diesel & 50.11 & 67721.0 & 48.11 & \\
\hline 1586 & $7 / 27 / 98$ & Diesel & 40.00 & 77977.0 & 38.40 & \\
\hline 1592 & $7 / 27 / 98$ & Diesel & 39.23 & 48657.0 & 37.66 & \\
\hline 1593 & $7 / 27 / 98$ & Diesel & 38.03 & 67962.0 & 36.51 & \\
\hline 1586 & $7 / 28 / 98$ & Diesel & 61.99 & 78431.0 & 60.13 & \\
\hline 1592 & $7 / 28 / 98$ & Diesel & 38.99 & 48919.0 & 37.82 & \\
\hline 1593 & $7 / 28 / 98$ & Diesel & 39.03 & 68242.0 & 37.86 & \\
\hline 1586 & $7 / 29 / 98$ & Diesel & 31.73 & 78696.0 & 31.10 & \\
\hline 1592 & $7 / 29 / 98$ & Diesel & 27.85 & 49124.0 & 27.01 & \\
\hline 1593 & $7 / 29 / 98$ & Diesel & 39.02 & 68529.0 & 38.24 & \\
\hline 1586 & $7 / 30 / 98$ & Diesel & 51.76 & 79019.0 & 50.72 & \\
\hline 1592 & $7 / 30 / 98$ & Diesel & 32.76 & 49349.0 & 32.10 & \\
\hline 1593 & $7 / 30 / 98$ & Diesel & 40.23 & 68820.0 & 39.43 & \\
\hline 1586 & $7 / 31 / 98$ & Diesel & 45.85 & 79325.0 & 44.93 & \\
\hline 1592 & $7 / 31 / 98$ & Diesel & 32.93 & 49564.0 & 32.27 & \\
\hline 1593 & $7 / 31 / 98$ & Diesel & 34.79 & 69102.0 & 34.09 & 0.98 \\
\hline
\end{tabular}




\begin{tabular}{|c|c|c|c|c|c|c|}
\hline Truck & Date & Fuel & Amount & Odometer & Cost & Monthly Avg \\
\hline 1592 & $8 / 1 / 98$ & Diesel & 23.12 & 49759.8 & 22.66 & \\
\hline 1593 & $8 / 1 / 98$ & Diesel & 37.23 & 69344.4 & 36.49 & \\
\hline 1586 & $8 / 3 / 98$ & Diesel & 25.01 & 79571.0 & 25.01 & \\
\hline 1592 & $8 / 3 / 98$ & Diesel & 28.94 & 49981.0 & 28.94 & \\
\hline 1593 & $8 / 3 / 98$ & Diesel & 44.47 & 69600.0 & 44.47 & \\
\hline 1592 & $8 / 4 / 98$ & Diesel & 35.90 & 50194.0 & 35.90 & \\
\hline 1593 & $8 / 4 / 98$ & |Diesel & 31.60 & 69868.0 & 31.60 & \\
\hline 1586 & $8 / 5 / 98$ & Diesel & 32.81 & 79788.0 & 33.14 & \\
\hline 1592 & $8 / 5 / 98$ & Diesel & 26.90 & 50393.0 & 26.55 & \\
\hline 1593 & $8 / 5 / 98$ & Diesel & 36.40 & 70141.0 & 36.76 & \\
\hline 1586 & $8 / 6 / 98$ & Diesel & 28.70 & 80020.0 & 28.99 & \\
\hline 1593 & $8 / 6 / 98$ & Diesel & 46.35 & 70348.0 & 46.81 & \\
\hline 1592 & $8 / 7 / 98$ & Diesel & 62.26 & 50840.0 & 62.88 & \\
\hline 1593 & $8 / 7 / 98$ & Diesel & 40.99 & 70726.0 & 41.40 & \\
\hline 1586 & $8 / 8 / 98$ & Diesel & 72.04 & 80516.0 & 72.76 & \\
\hline 1593 & $8 / 8 / 98$ & Diesel & 36.47 & 70940.0 & 36.83 & \\
\hline 1586 & $8 / 10 / 98$ & Diesel & 38.22 & 80871.0 & 38.60 & \\
\hline 1592 & $8 / 10 / 98$ & Diesel & 75.00 & 51357.0 & 75.75 & \\
\hline 1593 & $8 / 10 / 98$ & Diesel & 39.43 & 71428.0 & 39.82 & \\
\hline 1586 & $8 / 11 / 98$ & Diesel & 34.98 & 81117.0 & 35.33 & \\
\hline 1592 & $8 / 11 / 98$ & Diesel & 21.91 & 51563.0 & 21.91 & \\
\hline 1593 & $8 / 11 / 98$ & Diesel & 27.00 & 71640.0 & 27.00 & \\
\hline 1592 & $8 / 12 / 98$ & Diesel & 31.53 & 51771.0 & 31.53 & \\
\hline 1593 & $8 / 12 / 98$ & Diesel & 29.98 & 71918.0 & 29.98 & \\
\hline 1586 & $8 / 13 / 98$ & Diesel & 54.47 & 81377.0 & 54.47 & \\
\hline 1592 & $8 / 13 / 98$ & Diesel & 30.39 & 51979.0 & 30.39 & \\
\hline 1593 & $8 / 13 / 98$ & Diesel & 39.33 & 72206.0 & 39.33 & \\
\hline 1586 & $8 / 14 / 98$ & Diesel & 49.08 & 81719.0 & 49.08 & \\
\hline 1592 & $8 / 14 / 98$ & Diesel & 41.76 & 52262.0 & 41.76 & \\
\hline 1593 & $8 / 14 / 98$ & Diesel & 46.91 & 72499.0 & 46.91 & \\
\hline 1593 & $8 / 15 / 98$ & Diesel & 28.59 & 72272.0 & 28.59 & \\
\hline 1586 & $8 / 15 / 98$ & Diesel & 29.03 & 81961.0 & 29.03 & \\
\hline 1592 & $8 / 15 / 98$ & Diesel & 33.07 & 52516.0 & 33.07 & \\
\hline 1586 & $8 / 15 / 98$ & Diesel & 41.01 & 82273.0 & 41.01 & \\
\hline 1586 & $8 / 17 / 98$ & Diesel & 42.98 & 82565.0 & 42.55 & \\
\hline 1592 & $8 / 17 / 98$ & Diesel & 25.58 & 52710.0 & 25.32 & \\
\hline 1593 & $8 / 17 / 98$ & Diesel & 40.32 & 72994.0 & 40.32 & \\
\hline 1586 & $8 / 18 / 98$ & Diesel & 25.65 & 82760.0 & 25.39 & \\
\hline 1592 & $8 / 18 / 98$ & Diesel & 36.14 & 52931.0 & 35.78 & \\
\hline 1593 & $8 / 18 / 98$ & Diesel & 30.97 & 73204.0 & 30.66 & \\
\hline 1586 & $8 / 19 / 98$ & Diesel & 27.23 & 82936.0 & 26.96 & \\
\hline 1592 & $8 / 19 / 98$ & Diesel & 34.20 & 53136.0 & 33.86 & \\
\hline 1593 & $8 / 19 / 98$ & Diesel & 37.39 & 73506.0 & 37.02 & \\
\hline 1586 & $8 / 20 / 98$ & Diesel & 23.22 & 83133.0 & 22.99 & \\
\hline 1592 & $8 / 20 / 98$ & Diesel & 21.83 & 53335.0 & 21.61 & \\
\hline 1593 & $8 / 20 / 98$ & Diesel & 32.02 & 73828.0 & 31.70 & \\
\hline 1586 & $8 / 21 / 98$ & Diesel & 54.03 & 83478.0 & 52.95 & \\
\hline 1592 & $8 / 21 / 98$ & Diesel & 24.60 & 53605.0 & 24.11 & \\
\hline 1593 & $8 / 21 / 98$ & Diesel & 42.88 & 74010.0 & 42.02 & \\
\hline 1586 & $8 / 22 / 98$ & Diesel & 37.49 & 83775.0 & 36.74 & \\
\hline 1592 & $8 / 22 / 98$ & Diesel & 48.24 & 53838.0 & 47.28 & \\
\hline 1593 & $8 / 22 / 98$ & Diesel & 35.01 & 74393.0 & 34.31 & \\
\hline 1586 & $8 / 24 / 98$ & Diesel & 24.35 & 83966.0 & 23.86 & \\
\hline 1592 & $8 / 24 / 98$ & Diesel & 24.41 & 54030.0 & 23.92 & \\
\hline
\end{tabular}




\begin{tabular}{|c|c|c|c|c|c|c|}
\hline Truck & Date & Fuel & Amount & Odometer & Cost & Monthly Avg \\
\hline 1593 & $8 / 24 / 98$ & Diesel & 37.08 & 74874.0 & 36.34 & \\
\hline 1586 & $8 / 25 / 98$ & Diesel & 52.81 & 84330.0 & 51.75 & \\
\hline 1593 & $8 / 25 / 98$ & Diesel & 31.15 & 74881.0 & 30.53 & \\
\hline 1586 & $8 / 26 / 98$ & Diesel & 34.22 & 84563.0 & 33.54 & \\
\hline 1592 & $8 / 26 / 98$ & Diesel & 54.90 & 54374.0 & 53.80 & \\
\hline 1593 & $8 / 26 / 98$ & Diesel & 47.92 & 75178.0 & 46.96 & \\
\hline 1586 & $8 / 27 / 98$ & Diesel & 38.10 & 84883.0 & 37.34 & \\
\hline 1592 & $8 / 27 / 98$ & Diesel & 25.70 & 54579.0 & 25.19 & \\
\hline 1593 & $8 / 27 / 98$ & Diesel & 31.91 & 75458.0 & 31.27 & \\
\hline 1592 & $8 / 28 / 98$ & Diesel & 28.02 & 54797.0 & 27.46 & \\
\hline 1593 & $8 / 28 / 98$ & Diesel & 36.97 & 75747.0 & 36.23 & \\
\hline 1593 & $8 / 28 / 98$ & Diesel & 16.60 & 75878.0 & 16.27 & \\
\hline 1586 & $8 / 29 / 98$ & Diesel & 54.64 & 85354.0 & 53.55 & \\
\hline 1592 & $8 / 29 / 98$ & Diesel & 45.25 & 55083.0 & 44.35 & \\
\hline 1586 & $8 / 31 / 98$ & Diesel & 17.34 & 85937.0 & 16.99 & \\
\hline 1592 & $8 / 31 / 98$ & Diesel & 45.31 & 55268.0 & 44.40 & \\
\hline 1593 & $8 / 31 / 98$ & Diesel & 37.08 & 76137.0 & 36.34 & 0.99 \\
\hline 1592 & $9 / 1 / 98$ & Diesel & 38.39 & 55366.0 & 37.62 & \\
\hline 1593 & $9 / 1 / 98$ & Diesel & 28.25 & 76344.0 & 27.69 & \\
\hline 1592 & $9 / 2 / 98$ & Diesel & 21.86 & 55570.0 & 21.42 & \\
\hline 1593 & $9 / 2 / 98$ & Diesel & 29.68 & 76631.0 & 29.09 & \\
\hline 1586 & $9 / 3 / 98$ & Diesel & 41.15 & 85716.0 & 40.33 & \\
\hline 1592 & $9 / 3 / 98$ & Diesel & 27.05 & 55774.0 & 26.51 & \\
\hline 1593 & $9 / 3 / 98$ & Diesel & 31.61 & 76903.0 & 30.98 & \\
\hline 1592 & $9 / 4 / 98$ & Diesel & 49.35 & 56106.0 & 49.35 & \\
\hline 1593 & $9 / 4 / 98$ & Diesel & 42.83 & 77170.0 & 42.83 & \\
\hline 1586 & $9 / 5 / 98$ & Diesel & 52.01 & 86058.0 & 52.01 & \\
\hline 1586 & $9 / 5 / 98$ & Diesel & 40.74 & 86344.0 & 40.74 & \\
\hline 1592 & $9 / 5 / 98$ & Diesel & 13.53 & 56218.0 & 13.53 & \\
\hline 1593 & $9 / 5 / 98$ & Diesel & 33.53 & 77408.0 & 33.53 & \\
\hline 1586 & $9 / 7 / 98$ & Diesel & 47.53 & 86658.0 & 47.52 & \\
\hline 1592 & $9 / 7 / 98$ & Diesel & 31.46 & 56422.0 & 31.46 & \\
\hline 1593 & $9 / 7 / 98$ & Diesel & 38.88 & 77682.0 & 38.88 & \\
\hline 1586 & $9 / 8 / 98$ & Diesel & 36.09 & 86903.0 & 36.09 & \\
\hline 1586 & $9 / 9 / 98$ & Diesel & 28.48 & 87091.0 & 28.76 & \\
\hline 1593 & $9 / 9 / 98$ & Diesel & 40.99 & 77962.0 & 41.40 & \\
\hline 1586 & $9 / 10 / 98$ & Diesel & 40.70 & 87418.0 & 41.51 & \\
\hline 1592 & $9 / 10 / 98$ & Diesel & 32.60 & 56640.0 & 33.25 & \\
\hline 1593 & $9 / 10 / 98$ & Diesel & 34.30 & 78243.0 & 34.99 & \\
\hline 1592 & $9 / 11 / 98$ & Diese! & 21.92 & 56788.0 & 22.80 & \\
\hline 1593 & $9 / 11 / 98$ & Diesel & 36.27 & 78524.0 & 37.72 & \\
\hline 1593 & $9 / 11 / 98$ & Diesel & 4.99 & 78524.0 & 5.19 & \\
\hline 1586 & $9 / 11 / 98$ & Diesel & 38.61 & 87747.0 & 40.15 & \\
\hline 1586 & $9 / 12 / 98$ & Diesel & 41.85 & 88032.0 & 43.52 & \\
\hline 1592 & $9 / 12 / 98$ & Diesel & 27.49 & 56973.0 & 28.59 & \\
\hline 1593 & $9 / 12 / 98$ & Diesel & 36.87 & 78792.0 & 38.34 & \\
\hline 1586 & $9 / 14 / 98$ & Diesel & 43.81 & 88341.0 & 45.56 & \\
\hline 1592 & $9 / 14 / 98$ & Diesel & 32.68 & 57178.0 & 33.99 & \\
\hline 1593 & $9 / 14 / 98$ & Diesel & 39.27 & 79058.0 & 40.84 & \\
\hline 1592 & $9 / 15 / 98$ & Diesel & 35.95 & 57432.0 & 37.39 & \\
\hline 1586 & $9 / 16 / 98$ & Diesel & 24.23 & 88517.0 & 25.20 & \\
\hline 1592 & $9 / 16 / 98$ & Diesel & 21.52 & 57637.0 & 22.38 & \\
\hline 1593 & $9 / 16 / 98$ & Diesel & 40.22 & 79356.0 & 41.83 & \\
\hline 1586 & $9 / 17 / 98$ & Diesel & 4.56 & 88826.0 & 4.74 & \\
\hline
\end{tabular}




\begin{tabular}{|c|c|c|c|c|c|c|}
\hline Truck & Date & Fuel & Amount & Odometer & Cost & Monthly Avg \\
\hline 1592 & $9 / 17 / 98$ & Diesel & 34.23 & 57845.0 & 35.60 & \\
\hline 1593 & $9 / 17 / 98$ & Diesel & 35.43 & 79647.0 & 36.85 & \\
\hline 1586 & $9 / 17 / 98$ & Diesel & 36.40 & 88826.0 & 37.86 & \\
\hline 1586 & $9 / 18 / 98$ & Diesel & 45.13 & 89157.0 & 46.03 & \\
\hline 1593 & $9 / 18 / 98$ & Diesel & 36.65 & 79968.0 & 37.38 & \\
\hline 1592 & $9 / 19 / 98$ & Diesel & 13.30 & 57958.0 & 13.57 & \\
\hline 1593 & $9 / 19 / 98$ & Diesel & 32.68 & 80133.0 & 33.33 & \\
\hline 1586 & $9 / 21 / 98$ & Diesel & 45.55 & 89854.0 & 46.46 & \\
\hline 1592 & $9 / 21 / 98$ & Diesel & 42.90 & 58214.0 & 43.76 & \\
\hline 1593 & $9 / 21 / 98$ & Diesel & 35.03 & 80391.0 & 35.73 & \\
\hline 1586 & $9 / 22 / 98$ & Diesel & 29.63 & 90061.0 & 30.52 & \\
\hline 1592 & $9 / 22 / 98$ & Diesel & 27.54 & 58491.0 & 28.37 & \\
\hline 1586 & $9 / 23 / 98$ & Diesel & 25.40 & 90238.0 & 26.16 & \\
\hline 1592 & $9 / 23 / 98$ & Diesel & 32.93 & 58627.0 & 33.92 & \\
\hline 1593 & $9 / 23 / 98$ & Diesel & 36.19 & 80668.0 & 37.28 & \\
\hline 1586 & $9 / 24 / 98$ & Diesel & 40.71 & 90563.0 & 41.52 & \\
\hline 1592 & $9 / 24 / 98$ & Diesel & 24.57 & 58834.0 & 25.06 & \\
\hline 1593 & $9 / 24 / 98$ & Diesel & 35.60 & 80942.0 & 36.31 & \\
\hline 1586 & $9 / 25 / 98$ & Diesel & 46.54 & 90912.0 & 47.94 & \\
\hline 1592 & $9 / 25 / 98$ & Diesel & 29.03 & 59029.0 & 29.90 & \\
\hline 1593 & $9 / 25 / 98$ & Diesel & 39.81 & 81238.0 & 41.00 & \\
\hline 1586 & $9 / 26 / 98$ & Diesel & 49.74 & 91227.0 & 51.23 & \\
\hline 1586 & $9 / 26 / 98$ & Diesel & 30.26 & 91479.0 & 31.47 & \\
\hline 1592 & $9 / 26 / 98$ & Diesel & 38.92 & 59319.0 & 40.09 & \\
\hline 1593 & $9 / 26 / 98$ & Diesel & 36.42 & 81509.0 & 37.51 & \\
\hline 1592 & $9 / 28 / 98$ & Diesel & 34.23 & 59531.0 & 35.60 & \\
\hline 1593 & $9 / 28 / 98$ & Diesel & 35.48 & 81765.0 & 36.90 & \\
\hline 1586 & $9 / 29 / 98$ & Diesel & 38.24 & 91756.0 & 39.39 & \\
\hline 1592 & $9 / 29 / 98$ & Diesel & 27.30 & 59739.0 & 28.12 & \\
\hline 1593 & $9 / 29 / 98$ & Diesel & 35.04 & 82207.0 & 36.09 & \\
\hline 1586 & $9 / 30 / 98$ & Diesel & 25.72 & 91968.0 & 26.49 & \\
\hline 1592 & $9 / 30 / 98$ & Diesel & 28.92 & 59950.0 & 29.79 & \\
\hline 1593 & $9 / 30 / 98$ & Diesel & 37.12 & 82300.0 & 38.23 & 1.02 \\
\hline 1592 & $10 / 1 / 98$ & Diesel & 35.68 & 60203.0 & 36.75 & \\
\hline 1593 & $10 / 1 / 98$ & Diesel & 40.19 & 82617.0 & 41.40 & \\
\hline 1586 & $10 / 1 / 98$ & Diesel & 46.95 & 92295.0 & 48.36 & \\
\hline 1586 & $10 / 2 / 98$ & Diesel & 47.06 & 92633.0 & 48.47 & \\
\hline 1593 & $10 / 2 / 98$ & Diesel & 45.40 & 82923.0 & 46.76 & \\
\hline 1592 & $10 / 5 / 98$ & Diesel & 19.17 & 60658.0 & 19.74 & \\
\hline 1593 & $10 / 5 / 98$ & Diesel & 38.78 & 83205.0 & 39.94 & \\
\hline 1592 & $10 / 6 / 98$ & Diesel & 34.59 & 61080.0 & 34.94 & \\
\hline 1586 & $10 / 7 / 98$ & Diesel & 30.17 & 93076.0 & 30.17 & \\
\hline 1592 & $10 / 7 / 98$ & Diesel & 28.38 & 61084.0 & 28.38 & \\
\hline 1592 & $10 / 7 / 98$ & Diesel & 3.93 & 61085.0 & 3.93 & \\
\hline 1593 & $10 / 7 / 98$ & Diesel & 44.01 & 83492.0 & 44.01 & \\
\hline 1586 & $10 / 8 / 98$ & Diesel & 41.53 & 93407.0 & 41.11 & \\
\hline 1592 & $10 / 8 / 98$ & Diesel & 28.91 & 61302.0 & 28.62 & \\
\hline 1593 & $10 / 8 / 98$ & Diesel & 31.69 & 83761.0 & 31.37 & \\
\hline 1586 & $10 / 9 / 98$ & Diesel & 52.00 & 93753.0 & 51.48 & \\
\hline 1592 & $10 / 9 / 98$ & Diesel & 24.16 & 61471.0 & 23.92 & \\
\hline 1593 & $10 / 9 / 98$ & Diesel & 38.53 & 84056.0 & 38.14 & \\
\hline 1586 & $10 / 12 / 98$ & Diesel & 18.64 & 94164.0 & 18.45 & \\
\hline 1592 & $10 / 12 / 98$ & Diesel & 36.78 & 61680.0 & 36.41 & \\
\hline 1592 & $10 / 13 / 98$ & Diesel & 18.32 & 61900.0 & 18.41 & \\
\hline
\end{tabular}




\begin{tabular}{|c|c|c|c|c|c|c|}
\hline Truck & Date & Fuel & Amount & Odometer & Cost & Monthly Avg \\
\hline \begin{tabular}{|l|}
1592 \\
\end{tabular} & $10 / 14 / 98$ & Diesel & 34.24 & 62026.0 & 33.90 & \\
\hline 1593 & $10 / 14 / 98$ & Diesel & 23.84 & 84335.0 & 23.60 & \\
\hline 1586 & $10 / 15 / 98$ & Diesel & 50.47 & 94519.0 & 49.97 & \\
\hline 1592 & $10 / 15 / 98$ & Diesel & 34.96 & 62302.0 & 34.61 & \\
\hline 1593 & $10 / 15 / 98$ & Diesel & 46.98 & 84612.0 & 46.51 & \\
\hline 1593 & $10 / 16 / 98$ & Diesel & 45.11 & 84499.0 & 44.66 & \\
\hline 1592 & $10 / 16 / 98$ & Diesel & 24.41 & 62462.0 & 24.17 & \\
\hline 1586 & $10 / 17 / 98$ & Diesel & 46.93 & 94848.0 & 46.46 & \\
\hline 1586 & $10 / 17 / 98$ & Diesel & 44.28 & 95125.0 & 43.84 & \\
\hline 1592 & $10 / 17 / 98$ & Diesel & 47.22 & 62763.0 & 46.75 & \\
\hline 1592 & $10 / 19 / 98$ & Diesel & 29.98 & 62976.0 & 29.98 & \\
\hline 1593 & $10 / 19 / 98$ & Diesel & 36.82 & 85167.0 & 36.82 & \\
\hline 1586 & $10 / 20 / 98$ & Diesel & 39.13 & 95659.0 & 39.13 & \\
\hline 1592 & $10 / 20 / 98$ & Diesel & 29.66 & 63186.0 & 29.66 & \\
\hline 1586 & $10 / 20 / 98$ & Diesel & 44.81 & 95407.0 & 44.81 & \\
\hline 1586 & $10 / 21 / 98$ & Diesel & 29.73 & 95864.0 & 29.73 & \\
\hline 1592 & $10 / 21 / 98$ & Diesel & 20.46 & 63392.0 & 20.46 & \\
\hline 1593 & $10 / 21 / 98$ & Diesel & 36.73 & 85455.0 & 36.73 & \\
\hline 1586 & $10 / 22 / 98$ & Diesel & 41.47 & 96185.0 & 41.47 & \\
\hline 1592 & $10 / 22 / 98$ & Diesel & 27.92 & 63602.0 & 27.92 & \\
\hline 1593 & $10 / 22 / 98$ & Diesel & 30.17 & 85739.0 & 30.17 & \\
\hline 1586 & $10 / 23 / 98$ & Diesel & 44.56 & 96503.0 & 44.56 & \\
\hline 1592 & $10 / 23 / 98$ & Diesel & 49.58 & 63934.0 & 49.58 & \\
\hline 1593 & $10 / 23 / 98$ & Diesel & 36.47 & 86019.0 & 36.47 & \\
\hline 1586 & $10 / 24 / 98$ & Diesel & 47.95 & 96788.0 & 47.95 & \\
\hline 1592 & $10 / 26 / 98$ & Diesel & 34.31 & 64188.0 & 34.31 & \\
\hline 1586 & $10 / 27 / 98$ & Diesel & 30.36 & 97294.0 & 30.66 & \\
\hline 1592 & $10 / 27 / 98$ & Diesel & 25.23 & 64391.0 & 25.48 & \\
\hline 1593 & $10 / 27 / 98$ & Diesel & 33.96 & 86228.0 & 34.30 & \\
\hline 1586 & $10 / 28 / 98$ & Diesel & 28.72 & 97482.0 & 29.01 & \\
\hline 1592 & $10 / 28 / 98$ & Diesel & 37.00 & 64648.0 & 37.37 & \\
\hline 1593 & $10 / 28 / 98$ & Diesel & 36.28 & 86506.0 & 36.64 & \\
\hline 1592 & $10 / 29 / 98$ & Diesel & 29.48 & 64856.0 & 29.77 & \\
\hline 1593 & $10 / 29 / 98$ & Diesel & 36.55 & 86767.0 & 36.92 & \\
\hline 1586 & $10 / 30 / 98$ & Diesel & 48.18 & 97811.0 & 48.66 & \\
\hline 1586 & $10 / 30 / 98$ & Diesel & 46.63 & 98120.0 & 47.10 & \\
\hline 1592 & $10 / 30 / 98$ & Diesel & 24.05 & 65047.0 & 24.29 & \\
\hline 1593 & $10 / 30 / 98$ & Diesel & 39.10 & 87069.0 & 39.49 & \\
\hline 1586 & $10 / 31 / 98$ & Diesel & 25.22 & 98443.0 & 25.47 & \\
\hline 1586 & $10 / 31 / 98$ & Diesel & 48.95 & 98728.0 & 49.44 & \\
\hline 1592 & $10 / 31 / 98$ & Diesel & 18.94 & 65171.0 & 19.13 & 1.00 \\
\hline 1586 & $11 / 3 / 98$ & Diesel & 44.84 & 98994.0 & 45.29 & \\
\hline 1592 & $11 / 3 / 98$ & Diesel & 26.43 & 65705.0 & 26.69 & \\
\hline 1593 & $11 / 3 / 98$ & Diesel & 44.46 & 87663.0 & 44.90 & \\
\hline 1586 & $11 / 4 / 98$ & Diesel & 24.89 & 99169.0 & 25.14 & \\
\hline 1592 & $11 / 4 / 98$ & Diesel & 27.52 & 65921.0 & 27.80 & \\
\hline 1593 & $11 / 4 / 98$ & Diesel & 38.71 & 87957.0 & 39.10 & \\
\hline 1586 & $11 / 5 / 98$ & Diesel & 39.88 & 99491.0 & 40.28 & \\
\hline 1592 & $11 / 5 / 98$ & Diesel & 25.62 & 66125.0 & 25.88 & \\
\hline 1593 & $11 / 5 / 98$ & Diesel & 34.99 & 88221.0 & 35.34 & \\
\hline 1586 & $11 / 6 / 98$ & Diesel & 39.42 & 99817.0 & 40.21 & \\
\hline 1592 & $11 / 6 / 98$ & Diesel & 32.32 & 66373.0 & 32.97 & \\
\hline 1593 & $11 / 6 / 98$ & Diesel & 32.90 & 88496.0 & 33.56 & \\
\hline 1586 & $11 / 7 / 98$ & Diesel & 52.12 & 100094.0 & 53.16 & \\
\hline
\end{tabular}




\begin{tabular}{|c|c|c|c|c|c|c|}
\hline Truck & Date & Fuel & Amount & Odometer & Cost & Monthiy Avg \\
\hline 1593 & $11 / 7 / 98$ & Diesel & 35.15 & 88669.0 & 35.85 & \\
\hline 1586 & $11 / 9 / 98$ & Diesel & 53.21 & 100383.0 & 54.27 & \\
\hline 1593 & $11 / 9 / 98$ & Diesel & 36.28 & 88942.0 & 37.01 & \\
\hline 1592 & $11 / 9 / 98$ & Diesel & 37.00 & 66566.0 & 37.74 & \\
\hline 1586 & $11 / 10 / 98$ & Diesel & 20.13 & 100495.0 & 20.33 & \\
\hline 1592 & $11 / 10 / 98$ & Diesel & 25.84 & 66773.0 & 26.10 & \\
\hline 1593 & $11 / 10 / 98$ & Diesel & 39.62 & 89234.0 & 40.02 & \\
\hline 1586 & $11 / 11 / 98$ & Diesel & 23.87 & 100671.0 & 24.11 & \\
\hline 1592 & $11 / 11 / 98$ & Diesel & 31.04 & 66976.0 & 31.35 & \\
\hline 1593 & $11 / 11 / 98$ & Diesel & 39.26 & 89512.0 & 39.65 & \\
\hline 1586 & $11 / 12 / 98$ & Diesel & 25.08 & 100859.0 & 25.33 & \\
\hline 1592 & $11 / 12 / 98$ & Diesel & 23.34 & 67179.0 & 23.57 & \\
\hline 1593 & $11 / 12 / 98$ & Diesel & 34.07 & 89797.0 & 34.41 & \\
\hline 1593 & $11 / 13 / 98$ & Diesel & 38.24 & 90072.0 & 38.62 & \\
\hline 1586 & $11 / 13 / 98$ & Diesel & 46.27 & 101179.0 & 46.73 & \\
\hline 1586 & $11 / 14 / 98$ & Diesel & 40.34 & 101458.0 & 40.74 & \\
\hline 1586 & $11 / 16 / 98$ & Diesel & 50.33 & 101747.0 & 49.83 & \\
\hline 1592 & $11 / 16 / 98$ & Diesel & 30.72 & 67391.0 & 30.41 & \\
\hline 1593 & $11 / 16 / 98$ & Diesel & 36.57 & 90358.0 & 36.20 & \\
\hline 1586 & $11 / 17 / 98$ & Diesel & 27.43 & 101984.0 & 26.88 & \\
\hline 1592 & $11 / 17 / 98$ & Diesel & 28.34 & 67958.0 & 27.77 & \\
\hline 1593 & $11 / 17 / 98$ & Diesel & 35.39 & 90609.0 & 34.68 & \\
\hline 1593 & $11 / 18 / 98$ & Diesel & 39.44 & 90883.0 & 38.65 & \\
\hline 1586 & $11 / 19 / 98$ & Diesel & 57.85 & 102220.0 & 56.69 & \\
\hline 1592 & $11 / 19 / 98$ & Diesel & 61.25 & 68008.0 & 60.03 & \\
\hline 1593 & $11 / 19 / 98$ & Diesel & 43.84 & 91156.0 & 42.96 & \\
\hline 1586 & $11 / 20 / 98$ & Diesel & 46.57 & 102688.0 & 44.71 & \\
\hline 1592 & $11 / 20 / 98$ & Diesel & 41.94 & 68336.0 & 40.26 & \\
\hline 1593 & $11 / 20 / 98$ & Diesel & 41.02 & 91434.0 & 39.38 & \\
\hline 1586 & $11 / 21 / 98$ & Diesel & 27.90 & 102900.0 & 26.78 & \\
\hline 1586 & $11 / 23 / 98$ & Diesel & 49.91 & 103071.0 & 47.41 & \\
\hline 1592 & $11 / 23 / 98$ & Diesel & 39.29 & 68745.0 & 37.33 & \\
\hline 1593 & $11 / 23 / 98$ & Diesel & 44.56 & 91600.0 & 42.33 & \\
\hline 1593 & $11 / 23 / 98$ & Diesel & 49.41 & 91800.0 & 46.94 & \\
\hline 1592 & $11 / 24 / 98$ & Diesel & 37.03 & 69000.0 & 35.18 & \\
\hline 1593 & $11 / 24 / 98$ & Diesel & 23.08 & 91951.0 & 21.93 & \\
\hline 1592 & $11 / 25 / 98$ & Diesel & 26.34 & 69209.0 & 25.02 & \\
\hline 1593 & $11 / 25 / 98$ & Diesel & 35.75 & 92274.0 & 33.96 & \\
\hline 1586 & $11 / 26 / 98$ & Diesel & 51.86 & 103434.0 & 49.26 & \\
\hline 1593 & $11 / 27 / 98$ & Diesel & 42.96 & 92508.0 & 40.81 & \\
\hline 1586 & $11 / 28 / 98$ & Diesel & 49.50 & 103770.0 & 47.03 & \\
\hline 1586 & $11 / 28 / 98$ & Diesel & 47.64 & 104049.0 & 45.26 & \\
\hline 1586 & $11 / 30 / 98$ & Diesel & 50.04 & 104347.0 & 47.04 & \\
\hline 1592 & $11 / 30 / 98$ & Diesel & 34.22 & 69418.0 & 32.17 & \\
\hline 1593 & $11 / 30 / 98$ & Diesel & 42.43 & 92785.0 & 39.88 & 0.99 \\
\hline 1593 & $12 / 1 / 98$ & Diesel & 35.25 & 93027.0 & 33.14 & \\
\hline 1592 & $12 / 2 / 98$ & Diesel & 28.10 & 69628.0 & 26.41 & \\
\hline 1593 & $12 / 2 / 98$ & Diesel & 26.95 & 93232.0 & 25.33 & \\
\hline 1586 & $12 / 2 / 98$ & Diesel & 30.26 & 104550.0 & 28.44 & \\
\hline 1586 & $12 / 3 / 98$ & Diesel & 46.82 & 104863.0 & 43.07 & \\
\hline 1592 & $12 / 3 / 98$ & Diesel & 0.10 & 69836.0 & 0.09 & \\
\hline 1592 & $12 / 3 / 98$ & Diesel & 37.11 & 69837.0 & 34.14 & \\
\hline 1593 & $12 / 3 / 98$ & Diesel & 38.04 & 93307.0 & 35.00 & \\
\hline 1586 & $12 / 4 / 98$ & Diesel & 48.50 & 105183.0 & 43.17 & \\
\hline
\end{tabular}




\begin{tabular}{|c|c|c|c|c|c|c|}
\hline Truck & Date & Fuel & Amount & Odometer & Cost & Monthly Avg \\
\hline 1593 & $12 / 4 / 98$ & Diesel & 44.04 & 93795.0 & 39.20 & \\
\hline 1592 & $12 / 7 / 98$ & Diesel & 31.58 & 70045.0 & 28.11 & \\
\hline 1593 & $12 / 7 / 98$ & Diesel & 40.19 & 94080.0 & 35.77 & \\
\hline 1592 & $12 / 8 / 98$ & Diesel & 33.43 & 70255.0 & 29.75 & \\
\hline 1593 & $12 / 8 / 98$ & Diesel & 56.71 & 94456.0 & 50.47 & \\
\hline 1586 & $12 / 9 / 98$ & Diesel & 26.59 & 105568.0 & 23.40 & \\
\hline 1592 & $12 / 9 / 98$ & Diesel & 29.86 & 70462.0 & 26.28 & \\
\hline 1593 & $12 / 9 / 98$ & Diesel & 45.15 & 94790.0 & 39.73 & \\
\hline 1586 & $12 / 10 / 98$ & Diesel & 42.20 & 105879.0 & 37.14 & \\
\hline 1592 & $12 / 10 / 98$ & Diesel & 24.40 & 70703.0 & 21.47 & \\
\hline 1593 & $12 / 10 / 98$ & Diesel & 32.54 & 95070.0 & 28.64 & \\
\hline 1586 & $12 / 11 / 98$ & Diesel & 43.89 & 106199.0 & 38.62 & \\
\hline 1593 & $12 / 11 / 98$ & Diesel & 41.81 & 95383.0 & 36.79 & \\
\hline 1592 & $12 / 14 / 98$ & Diesel & 44.73 & 70938.0 & 39.36 & \\
\hline 1593 & $12 / 14 / 98$ & Diesel & 45.44 & 95641.0 & 39.99 & \\
\hline 1586 & $12 / 15 / 98$ & Diesel & 37.26 & 106407.0 & 32.79 & \\
\hline 1592 & $12 / 15 / 98$ & Diesel & 27.79 & 71132.0 & 24.46 & \\
\hline 1593 & $12 / 15 / 98$ & Diesel & 44.70 & 95958.0 & 39.34 & \\
\hline 1586 & $12 / 16 / 98$ & Diesel & 30.02 & 106603.0 & 26.42 & \\
\hline 1592 & $12 / 16 / 98$ & Diesel & 32.28 & 71353.0 & 28.41 & \\
\hline 1593 & $12 / 16 / 98$ & Diesel & 38.30 & 96232.0 & 33.70 & \\
\hline 1586 & $12 / 17 / 98$ & Diesel & 0.44 & 106917.0 & 0.39 & \\
\hline 1586 & $12 / 17 / 98$ & Diesel & 0.01 & 106917.0 & 0.01 & \\
\hline 1586 & $12 / 17 / 98$ & Diesel & 39.88 & 106917.0 & 35.49 & \\
\hline 1592 & $12 / 17 / 98$ & Diesel & 33.79 & 71597.0 & 30.07 & \\
\hline 1593 & $12 / 17 / 98$ & Diesel & 38.58 & 96551.0 & 34.34 & \\
\hline 1586 & $12 / 18 / 98$ & Diesel & 44.01 & 107237.0 & 39.17 & \\
\hline 1593 & $12 / 18 / 98$ & Diesel & 37.02 & 96816.0 & 32.95 & \\
\hline 1586 & $12 / 20 / 98$ & Diesel & 0.15 & 107551.0 & 0.13 & \\
\hline 1586 & $12 / 21 / 98$ & Diesel & 46.41 & 107988.0 & 41.30 & \\
\hline 1592 & $12 / 21 / 98$ & Diesel & 37.89 & 71853.0 & 33.72 & \\
\hline 1593 & $12 / 21 / 98$ & Diesel & 47.34 & 97130.0 & 42.13 & \\
\hline 1586 & $12 / 22 / 98$ & Diesel & 42.16 & 108289.0 & 37.52 & \\
\hline 1592 & $12 / 22 / 98$ & Diesel & 29.03 & 72059.0 & 25.84 & \\
\hline 1593 & $12 / 22 / 98$ & Diesel & 28.94 & 97354.0 & 25.76 & \\
\hline 1586 & $12 / 23 / 98$ & Diesel & 35.02 & 108492.0 & 31.17 & \\
\hline 1592 & $12 / 23 / 98$ & Diesel & 36.15 & 72273.0 & 32.17 & \\
\hline 1593 & $12 / 23 / 98$ & Diesel & 42.79 & 97636.0 & 38.08 & \\
\hline 1586 & $12 / 28 / 98$ & Diesel & 45.98 & 109060.0 & 41.38 & \\
\hline 1592 & $12 / 28 / 98$ & Diesel & 33.17 & 72737.0 & 29.85 & \\
\hline 1593 & $12 / 28 / 98$ & Diesel & 30.55 & 98099.0 & 27.50 & \\
\hline 1586 & $12 / 29 / 98$ & Diesel & 34.15 & 109295.0 & 30.74 & \\
\hline 1592 & $12 / 29 / 98$ & Diesel & 35.38 & 72945.0 & 31.84 & \\
\hline 1593 & $12 / 29 / 98$ & Diesel & 24.49 & 98219.0 & 22.04 & \\
\hline 1586 & $12 / 30 / 98$ & Diesel & 30.02 & 109511.0 & 27.02 & \\
\hline 1592 & $12 / 30 / 98$ & Diesel & 29.35 & 73150.0 & 26.42 & \\
\hline 1593 & $12 / 30 / 98$ & Diesel & 41.13 & 98565.0 & 37.02 & \\
\hline 1592 & $12 / 31 / 98$ & Diesel & 43.51 & 73505.0 & 39.16 & \\
\hline 1593 & $12 / 31 / 98$ & Diesel & 24.70 & 98797.0 & 22.23 & \\
\hline \multirow[t]{4}{*}{1586} & $12 / 31 / 98$ & Diesel & 46.75 & 109792.0 & 42.08 & 0.89 \\
\hline & & & & & & \\
\hline & & Totals & 30208.60 & & 30419.90 & \\
\hline & & & & Average & 1.01 & \\
\hline
\end{tabular}




\section{Appendix G}

Monthly Maintenance Costs by Truck and Driver Complaint Summary 
Raley's Maintenance Costs by Vehicle System

\begin{tabular}{|c|c|c|c|c|c|c|c|c|c|c|c|c|c|c|c|c|}
\hline VMRS & Description & 1501 & 1502 & 1503 & 1504 & 1505 & 1506 & 1507 & 1508 & Total & Avg/Truck & 1586 & 1592 & 1593 & Total DSL & Avg/Truck \\
\hline 01 & A/C \& Heat & 23.36 & 0.00 & 72.16 & 63.80 & 60.00 & 0.00 & 0.00 & 43.80 & 263.12 & 32.89 & 83.47 & 0.00 & 15.00 & 98.47 & 32.82 \\
\hline $02 / 71$ & Cab \& Sheet Metal/Body & 401.25 & 101.62 & 479.85 & 380.50 & 301.00 & 235.66 & 437.72 & 165.50 & 2503.10 & 312.89 & 335.53 & 227.76 & 492.01 & 1055.30 & 351.77 \\
\hline 10 & Air System & 95.35 & 53.94 & 0.00 & 34.47 & 25.00 & 10.00 & 275.00 & 483.28 & 977.04 & 122.13 & 151.54 & 0.00 & 166.54 & 318.08 & 106.03 \\
\hline 11 & Front Axle & 0.00 & 0.00 & 0.00 & 0.00 & 0.00 & 18.50 & 0.00 & 0.00 & 18.50 & 2.31 & 95.00 & 0.00 & 95.00 & 190.00 & 63.33 \\
\hline 13 & Brakes & 0.00 & 0.00 & 0.00 & 0.00 & 0.00 & 0.00 & 30.00 & 0.00 & 30.00 & 3.75 & 25.00 & 0.00 & 133.60 & 158.60 & 52.87 \\
\hline 14 & Frame & 15.00 & 0.00 & 0.00 & 0.00 & 0.00 & 0.00 & 25.00 & 0.00 & 40.00 & 5.00 & 75.00 & 0.00 & 25.00 & 100.00 & 33.33 \\
\hline 15 & Steering & 0.00 & 50.00 & 0.00 & 0.00 & 0.00 & 0.00 & 0.00 & 0.00 & 50.00 & 6.25 & 0.00 & 0.00 & 0.00 & 0.00 & 0.00 \\
\hline 16 & Suspension & 20.00 & 20.00 & 90.32 & 20.00 & 20.00 & 106.01 & 20.00 & 116.01 & 412.34 & 51.54 & 80.32 & 20.00 & 20.00 & 120.32 & 40.11 \\
\hline 17 & Tires & 1960.00 & 1456.50 & 1350.00 & 360.00 & 1150.00 & 950.00 & 997.00 & 955.00 & 10178.50 & 272.31 & 670.00 & 595.00 & 835.00 & .00 & 3.33 \\
\hline 18 & Wheels & 0.00 & 0.00 & 0.00 & 0.00 & 0.00 & 0.00 & 1.64 & 0.00 & 1.64 & 0.21 & 0.00 & 0.00 & 0.00 & 0.00 & 0.00 \\
\hline 22 & Rear Axle & 0.00 & 0.00 & 0.00 & 0.00 & 0.00 & 0.00 & 0.00 & 0.00 & 0.00 & 0.00 & 0.00 & 0.00 & 0.00 & 0.00 & 0.00 \\
\hline 23 & Clutch & 18.77 & 0.00 & 0.00 & 0.00 & 0.00 & 0.00 & 0.00 & 0.00 & 18.77 & 2.35 & 0.00 & 0.00 & 0.00 & 0.00 & 0.00 \\
\hline 26 & sion & 0.00 & 0.00 & 0.00 & 0.00 & 0.00 & 0.00 & 0.00 & 0.00 & 0.00 & 0.00 & 0.00 & 0.00 & 0.00 & 0.00 & 0.00 \\
\hline 31 & Cha & 0.00 & 0.00 & 0.00 & 0.00 & 0.00 & 259.00 & 0.00 & 0.00 & 59.00 & 32.38 & 0.00 & 0.00 & 0.00 & 0.00 & 0.00 \\
\hline 32 & Crar & 160.90 & 228.85 & 348.35 & 413.85 & 228.85 & 627.85 & 507.70 & 0.00 & 2516.35 & 314.54 & 0.00 & 0.00 & 69.50 & 69.50 & 23.17 \\
\hline 33 & Ign & 327.24 & 163.62 & 327.24 & 327.24 & 1036.44 & 1036.44 & 1036.44 & 1036.44 & 5291.10 & 661.39 & 0.00 & 0.00 & 0.00 & 0.00 & 0.00 \\
\hline 34 & Lig & 198.12 & 199.52 & 69.27 & 189.73 & 224.82 & 72.26 & 363.57 & 129.47 & 1446.76 & 180.85 & 199.97 & 158.96 & 77.08 & 436.01 & 145.34 \\
\hline 42 & $\mathrm{CoO}$ & 0.00 & 65.45 & 6.18 & 9.27 & 21.18 & 0.00 & 27.36 & 6.18 & 135.62 & 6.95 & 0.00 & 34.27 & 0.00 & 34.27 & 11.42 \\
\hline 43 & Exhaust & 0.00 & 0.00 & 0.00 & 0.00 & 0.00 & 0.00 & 0.00 & 40.00 & 40.00 & 5.00 & 0.00 & 50.50 & 0.00 & 50.50 & 16.83 \\
\hline 44 & Fuel & 305.52 & 265.52 & 911.52 & 368.52 & 549.94 & 830.52 & 383.52 & 450.52 & 4065.58 & 508.20 & 26.60 & 26.60 & 458.33 & 511.53 & 170.51 \\
\hline 45 & Engin & 440.20 & 400.20 & 410.20 & 385.20 & 395.20 & 1354.26 & 410.20 & 385.20 & 4180.66 & 522.58 & 281.10 & 269.20 & 282.20 & 832.50 & 277.50 \\
\hline 50 & Accessories & 75.00 & 80.02 & 391.12 & 260.45 & 396.12 & 28.90 & 161.91 & 100.00 & 1493.52 & 186.69 & 68.49 & 160.82 & 334.70 & 564.01 & 188.00 \\
\hline 59 & Fifth Wheel & 90.00 & 50.00 & 592.00 & 0.00 & 32.76 & 0.00 & 0.00 & 0.00 & 764.76 & 95.60 & 0.00 & 0.00 & 0.00 & 0.00 & 0.00 \\
\hline 101 & Insp & 860.00 & 865.00 & 1030.00 & 940.00 & 900.00 & 880.00 & 925.00 & 880.00 & 7280.00 & 0.00 & 1000.00 & 1000.00 & 1000.00 & 3000.00 & 1000.00 \\
\hline & Total & 4990.71 & 4000.24 & 6078.21 & 4753.03 & 5341.31 & 6409.40 & 5602.06 & 4791.40 & 41966.36 & 5245.80 & 3092.02 & 2543.11 & 5003.96 & 10639.09 & 3546.36 \\
\hline
\end{tabular}




\begin{tabular}{|c|c|c|c|c|c|c|c|c|c|c|c|c|c|c|c|c|c|c|c|}
\hline Group & Sep-95 & Oct-95 & Nov-95 & Dec-95 & J2n-96 & Feb-96 & Mar-96 & Apr-96 & May-96 & Jun-96 & Jul-96 & Aug-96 & Sep-96 & Oet-96 & Nov-96 & Dec-96 & |2n-97| & Feb-97) & Mar-97 \\
\hline Mileage & 19,173 & 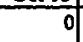 & & 57,065 & 0 & 18,601 & 35,299 & 0 & 20,532 & 34,309 & 19,978 & & 35,963 & 22,014 & 0 & 35,035 & 22,541 & 16,620 & 17,555 \\
\hline $\begin{array}{l}\text { No. of kC } \\
\text { Parts Cost }\end{array}$ & 165.93 & 0.00 & 0.00 & 856.80 & 0.00 & 109.92 & 363.86 & 0.00 & 133.71 & 386.29 & 610.31 & 95.00 & 962.76 & 991.42 & 0.00 & 584.36 & 466.91 & 326.69 & 194.04 \\
\hline Labor Hours & 11.9 & 0.0 & 0.0 & 323 & 0.0 & 10.9 & $\begin{array}{r}15.5 \\
0.37\end{array}$ & 0.0 & $\begin{array}{r}7.7 \\
0.725\end{array}$ & $\begin{array}{r}19.0 \\
0.39\end{array}$ & $\begin{array}{r}10.1 \\
0.056\end{array}$ & 0.0 & $\begin{array}{r}13.6 \\
0.046\end{array}$ & $\begin{array}{r}7.8 \\
0.063\end{array}$ & 0.0 & $\begin{array}{r}14.2 \\
0.037\end{array}$ & $\begin{array}{r}10.6 \\
0.044\end{array}$ & $\begin{array}{r}10.5 \\
0.051\end{array}$ & $\begin{array}{r}7.7 \\
0.033\end{array}$ \\
\hline $\begin{array}{l}\text { Total per Mile } \\
\text { Cum Tof per Mile }\end{array}$ & $\frac{0.040}{0.040}$ & 0.040 & 0.040 & $\frac{0.043}{0.042}$ & 0.042 & 0.035 & $\frac{0.032}{0.039}$ & 0.039 & $\frac{0.0203}{0.037}$ & $\begin{array}{l}0.0037 \\
0.037\end{array}$ & $\frac{0.030}{0.039}$ & 0.039 & 0.040 & 0.042 & 0.042 & 0.042 & 0.042 & 0.042 & 0.041 \\
\hline
\end{tabular}

\begin{tabular}{|c|c|c|c|c|c|c|c|c|c|c|c|c|c|c|c|c|c|c|c|c|c|c|c|c|}
\hline oup & \begin{tabular}{|c|} 
Diesel Co \\
Apr-97
\end{tabular} & May-97 & Jun-97 & Jul-97 & Aug-97 & Sep-97 & Oct.971 & \begin{tabular}{l|l|} 
Nov-97 & \\
\end{tabular} & Dec-97 & Jan-98 & Feb-98 & Mar-98 & Apr-98 & May-98 & Jun-98 & Jut-98 & \begin{tabular}{l|l|} 
Aug-98 \\
\end{tabular} & Sep-98 & Oet-98 & \begin{tabular}{l|l|} 
Nov-98 \\
\end{tabular} & \begin{tabular}{l|l|} 
Dec-98 \\
\end{tabular} & |an-99| & Total & PMA 2-3 \\
\hline Mileage & 17,779 & 17,032 & 18,423 & 20,587 & 19,171 & 18,078 & 17,507 & 17,995 & 15,907 & 17,651 & 16,094 & 19,787 & 16.564 & 17,633 & 21,928 & 24,679 & 41,959 & 0 & 0 & I & 0 & 0 & 693,459 & 110,201 \\
\hline $\begin{array}{l}\text { No. of RC } \\
\text { Parts Cost }\end{array}$ & 885.32 & 180.62 & 1033.85 & 1535.29 & 449.95 & 725.27 & 915.93 & 144.47 & 1286.12 & $\begin{array}{r}360.07 \\
10.1\end{array}$ & 438.40 & $\begin{array}{r}208.96 \\
8.5 \\
\end{array}$ & $\begin{array}{r}475.54 \\
7.2\end{array}$ & $\begin{array}{r}1356.62 \\
9.2\end{array}$ & $\begin{array}{r}827.26 \\
7.0\end{array}$ & $\begin{array}{r}411.76 \\
7.0\end{array}$ & $\begin{array}{r}1012.45 \\
17.6\end{array}$ & $\begin{array}{r}0.00 \\
0.0\end{array}$ & $\begin{array}{r}0.00 \\
0.0\end{array}$ & $\begin{array}{r}0.00 \\
0.0\end{array}$ & $\begin{array}{r}0.00 \\
0.0\end{array}$ & $\begin{array}{r}0.00 \\
0.0\end{array}$ & $\begin{array}{r}18,495.88 \\
322.7\end{array}$ & $\begin{array}{r}983.49 \\
55.5\end{array}$ \\
\hline $\begin{array}{l}\text { Labor Hours } \\
\text { Total per Mille }\end{array}$ & $\begin{array}{r}10.6 \\
0.080\end{array}$ & $\begin{array}{r}8.7 \\
0.036\end{array}$ & $\begin{array}{r}8.0 \\
0.078 \\
\end{array}$ & $\begin{array}{r}8.3 \\
0.095 \\
\end{array}$ & $\begin{array}{r}8.1 \\
0.045 \\
\end{array}$ & $\begin{array}{r}9.8 \\
0.067\end{array}$ & $\begin{array}{r}7.6 \\
0.074\end{array}$ & $\begin{array}{r}6.1 \\
0.025 \\
\end{array}$ & $\begin{array}{r}7.10 \\
0.105 \\
\end{array}$ & $\begin{array}{r}10.49 \\
0.049\end{array}$ & $\begin{array}{r}7.7 \\
0.057 \\
\end{array}$ & $\begin{array}{r}0.032 \\
0.032\end{array}$ & 0.050 & $\begin{array}{l}0.103 \\
\end{array}$ & 0.054 & 0.031 & 0.045 & & & & & & 0.050 & $\begin{array}{r}53.03 \\
0.034 \\
\end{array}$ \\
\hline Cum Tat per Mlle & 0.04 & 0.043 & 0.045 & 0.048 & 0.048 & 0.048 & 0.049 & 0.048 & 0.050 & 0.050 & 0.050 & 0.050 & 0.050 & 0.051 & 0.052 & 0.051 & 0.050 & 0.050 & 0.050 & 0.050 & 0.050 & 0.050 & & \\
\hline
\end{tabular}

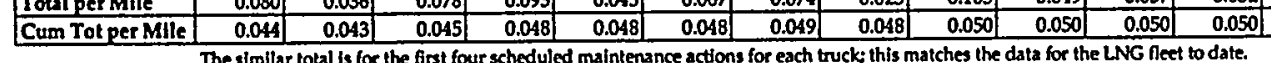

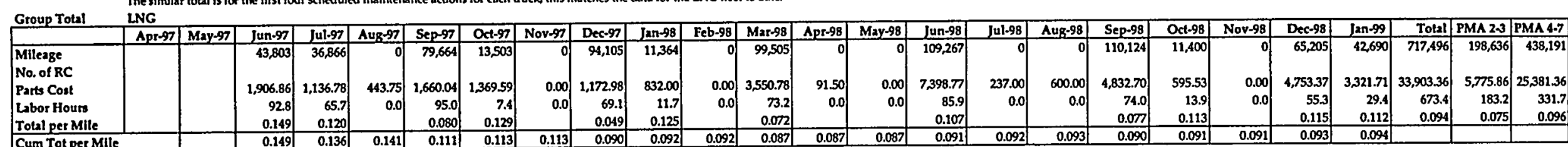

\section{Cum Tot per Mille}

\begin{tabular}{|c|c|c|c|c|c|c|c|c|c|c|c|c|c|c|c|c|c|c|c|}
\hline Ick 1586 & 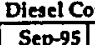 & $\frac{\text { ntrol }}{\text { Oet-95 }}$ & Nov-95 & Dec-95 & Jan-96 & Feb-96 & Mar-96 & Apr.96 & May-96 & Jun-96 & Jul-96 & $\begin{array}{l}\text { Aug-96 } \\
\end{array}$ & Sep-96 & Oct-96 & Nov.96 & Dec-96] & $\mid 2 n-97$ & Feb-97 & Mar-97 \\
\hline $\begin{array}{l}\text { Milexge } \\
\text { No. of RC }\end{array}$ & \begin{tabular}{|l|}
19,173 \\
\end{tabular} & & & 19,787 & & & 19,114 & & & 18,327 & & & 18,947 & & & 18,151 & & & \\
\hline $\begin{array}{l}\text { No. of RC } \\
\text { Parts Cost }\end{array}$ & 165.93 & & & 156.37 & & & 128.12 & & & 166.66 & & & 865.12 & & & 261.20 & & & \\
\hline Labor Hours & 11.9 & & & 12.1 & & & 5.9 & & & 9.7 & & & $\begin{array}{r}7.8 \\
0.6\end{array}$ & & & $\begin{array}{r}6.9 \\
0.033\end{array}$ & & & 0.033 \\
\hline
\end{tabular}

Truck 1586 Diesel Control (continued)

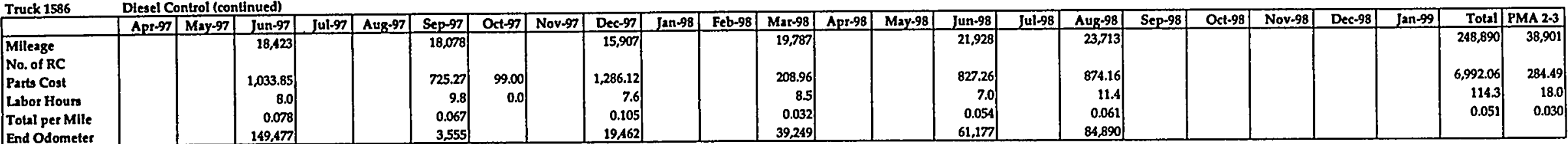

Diesel Control

\begin{tabular}{|c|c|c|c|c|c|c|c|c|c|c|c|c|c|c|c|c|c|c|c|}
\hline uck 1592 & Diesel C & ntrol & & & & & & & & & & & & & & & & & \\
\hline & Sep-95 & Oct-95 & Nov-95 & Dec-95 & Jan-96 & Feb-96 & \begin{tabular}{|l|} 
Mar-96 \\
\end{tabular} & Apr-96 & May-96 & Jun-96 & Jul-96 & Aug -96 & Sep-96 & Qet-96 & Nov-96 & Dec-96 & Jan- 97 & Feb-97 & Mar-9? \\
\hline $\begin{array}{l}\text { Mileage } \\
\text { No. of RC } \\
\text { Parts Cost } \\
\text { Labor Hours } \\
\text { Total per Mile }\end{array}$ & & & & \begin{tabular}{r|}
17,252 \\
\\
539.51 \\
12.3 \\
0.067 \\
17,252 \\
\end{tabular} & & & \begin{tabular}{r|}
16,185 \\
235.74 \\
9.6 \\
0.044 \\
33,437
\end{tabular} & & & \begin{tabular}{r|}
15,982 \\
219.63 \\
9.3 \\
0.043 \\
49,419 \\
\end{tabular} & & & $\begin{array}{r}17,016 \\
97.64 \\
5.8 \\
0.023 \\
66,435\end{array}$ & & & \begin{tabular}{r|}
16,884 \\
323.16 \\
7.3 \\
0.041 \\
83,319
\end{tabular} & & $\begin{array}{r}16,620 \\
326.69 \\
10.5 \\
0.051 \\
99,939\end{array}$ & \\
\hline
\end{tabular}


Through December 1998

\begin{tabular}{|c|c|c|c|c|c|c|c|c|c|c|c|c|c|c|c|c|c|c|c|c|c|c|c|c|}
\hline & Apr-97 & May-97 & Jun-97 & Jul-97 & Aug-97 & Sep-97 & Ock-97| & Nov-97 & Dec-97 & Jan-98 & Feb-98 & Marr-98 & Apr-98 & May-98 & $\begin{array}{ll}\text { Jun-98 } \\
\end{array}$ & Jul-98 & \begin{tabular}{|l|} 
Aug-98 \\
\end{tabular} & Sep-98 & Oct.98 & Nov.98 & Dec-98 & Jan-99 & Totill & 1 PMA ?-3 \\
\hline $\begin{array}{l}\text { Mileage } \\
\text { No. of RC } \\
\text { Parts Cost } \\
\text { Labor Hours } \\
\text { Total per Mile } \\
\text { End Odometer }\end{array}$ & & \begin{tabular}{|r|}
17,032 \\
\\
180.62 \\
8.7 \\
0.036 \\
116,971 \\
\end{tabular} & & & \begin{tabular}{r|}
19,171 \\
449.95 \\
8.1 \\
0.045 \\
136,142 \\
\end{tabular} & & & \begin{tabular}{r|}
17,995 \\
144.47 \\
6.1 \\
0.025 \\
2,131 \\
\end{tabular} & & & $\begin{array}{r}16,094 \\
438.40 \\
9.5 \\
0.057 \\
18,225 \\
\end{array}$ & & & \begin{tabular}{r|}
17.633 \\
$1,356.62$ \\
9.2 \\
0.103 \\
35,858 \\
\end{tabular} & & & $\begin{array}{r}18,246 \\
138.29 \\
6.2 \\
0.025 \\
54,104 \\
\end{array}$ & & & & 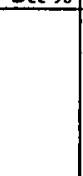 & 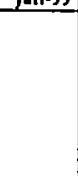 & \begin{tabular}{r|}
206,110 \\
$4,450,72$ \\
102.6 \\
0.046
\end{tabular} & $\begin{array}{r}32,167 \\
\\
455.37 \\
18.9 \\
0.044\end{array}$ \\
\hline
\end{tabular}

Truck 1593 Diesel Control

\begin{tabular}{|c|c|c|c|c|c|c|c|c|c|c|c|c|c|c|c|c|c|c|c|}
\hline & Sep-95 & Oct-95 & Nov-95 & Dec-95 & J2n-96 & Feb-96 & Mar-96 & Apr-96 & \begin{tabular}{|l|} 
May-96 \\
\end{tabular} & Jun-96 & Jul-96 & Aur-96 & Sep-96 & Oet-96 & Nov-96 & Dec.96 & Jan-97 & Feb-97 & Mar-97 \\
\hline $\begin{array}{l}\text { Mileage } \\
\text { No. of RC } \\
\text { Parts Cost } \\
\text { Labor Hours } \\
\text { Total per Mile } \\
\text { End Odometer }\end{array}$ & & & . & \begin{tabular}{r|}
20,026 \\
160.92 \\
7.9 \\
0.028 \\
20,026
\end{tabular} & & \begin{tabular}{r|}
18,601 \\
109.92 \\
10.9 \\
0.035 \\
38,627 \\
\end{tabular} & & & \begin{tabular}{r|}
20,532 \\
\\
133.71 \\
7.7 \\
0.025 \\
59,159
\end{tabular} & & \begin{tabular}{r|}
19,978 \\
\\
610.31 \\
10.1 \\
0.056 \\
79,137
\end{tabular} & $\begin{array}{r}95.00 \\
0.0\end{array}$ & & \begin{tabular}{r|}
22,014 \\
\\
991.42 \\
7.8 \\
0.063 \\
101,151
\end{tabular} & & & \begin{tabular}{r|}
22,541 \\
\\
466.91 \\
10.6 \\
0.044 \\
123.692
\end{tabular} & & \\
\hline
\end{tabular}

Truck 1593 Diesel Control (continued)

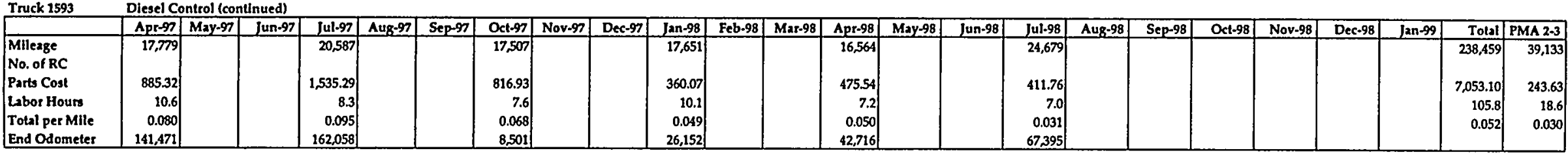

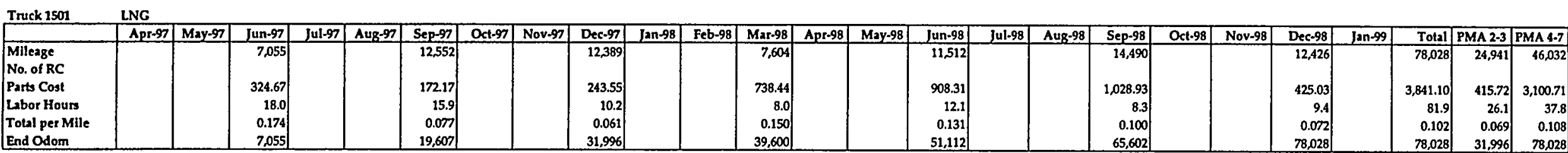

Truck 1502 LNG

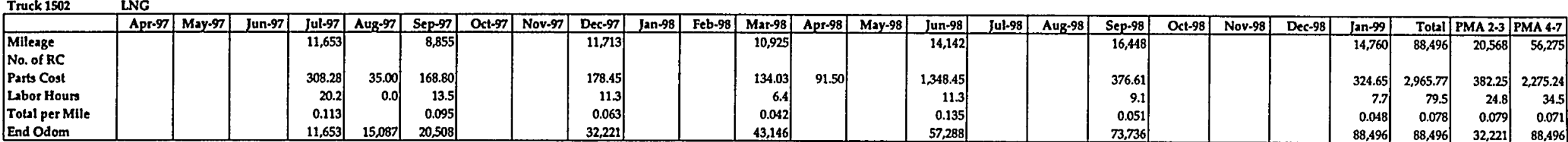

Toll permile

Truck 1503 LNC

\begin{tabular}{|c|c|c|c|c|c|c|c|c|c|c|c|c|c|c|c|c|c|c|c|c|c|c|c|c|c|}
\hline & Apr-97 & May-97 & Jun-97 & Jul-97 & $\begin{array}{l}\text { A } 48-97 \\
\end{array}$ & Sep-97| & Oct-97 & Nov-97 & Dec-97 & Jan-98 & Feb-98 & \begin{tabular}{|l|} 
Mar-98 \\
\end{tabular} & Apr-98 & May-98 & Jun-98 & Jul-98 & Aug.98 & Sep-98 & $\mathrm{OCt}_{\mathrm{Cl}}$ & Nov-98 & Dec-98 & Jan-99 & Total & PMA 2-3 & $\longdiv { \text { PMA 4.7 } }$ \\
\hline \begin{tabular}{|l|} 
Mileage \\
No. of RC
\end{tabular} & & & 7,618 & & & 12,505 & & & 14,415 & & & 14,905 & & & 14,803 & & & \begin{tabular}{|c|}
16,088 \\
\end{tabular} & & & 7,869 & & 88,203 & 26,920 & 53,665 \\
\hline Parts Cost & & & 230.28 & & 408.75 & 205.44 & & & 145.30 & & & 637.92 & & & $1,790.65$ & 180.00 & & 371.07 & & & 698.57 & & $4,667.98$ & 759.49 & 3,678 \\
\hline Labor Hours & & & $\begin{array}{r}19.5 \\
0.158\end{array}$ & & 0.0 & $\begin{array}{r}19.6 \\
\end{array}$ & & & $\begin{array}{r}11.2 \\
0.049\end{array}$ & & & $\begin{array}{r}11.4 \\
0.081\end{array}$ & & & $\begin{array}{r}12.1 \\
0.162\end{array}$ & & & 9.2 & & & 15.3 & & & 30.8 & 48.0 \\
\hline $\begin{array}{l}\text { Tolal per Mitle } \\
\text { End Odom }\end{array}$ & & & 7,618 & & 17,854 & 20,123 & & & $\begin{array}{r}0.049 \\
34,538\end{array}$ & & & $\begin{array}{r}0.001 \\
49,443\end{array}$ & & & $\begin{array}{r}0.104 \\
64,246\end{array}$ & & & $\begin{array}{r}0.032 \\
80,334\end{array}$ & & & $\begin{array}{r}0.186 \\
88,203\end{array}$ & & $\begin{array}{r}0.109 \\
88,203\end{array}$ & $\begin{array}{r}0.085 \\
34,538\end{array}$ & 88,26 \\
\hline
\end{tabular}


Through December 1998

\begin{tabular}{|c|c|c|c|c|c|c|c|c|c|c|c|c|c|c|c|c|c|c|c|c|c|c|c|c|c|}
\hline ruck $150 \mathrm{~A}$ & LNG & & & & & & & & & & & & & & & & & & & & & & & & \\
\hline & $A p R-97$ & \begin{tabular}{|l} 
May-97 \\
\end{tabular} & Jun-97 & Jul-97 & A ukg-97 & Sep-97| & Oet-97| & Nov-97 & Dec-97 & $J \geq n-A B$ & Feb-98 & Mar-98 & Apt-98 & May-98 & Jun-98 & Iul-98 & Aug-98 & Sep-98 & Oet-98 & Nov. 98 & Dec-98 & Jen-99| & Total & PMA 2.3 & PMA $4-7$ \\
\hline $\begin{array}{l}\text { Milieage } \\
\text { No. of RC } \\
\text { Parts Cost } \\
\text { Labor Houn } \\
\text { Total per Mile } \\
\text { End Odor }\end{array}$ & & & & \begin{tabular}{r|}
13,460 \\
200.55 \\
25.1 \\
0.115 \\
13,460
\end{tabular} & & $\begin{array}{r}13,012 \\
18658 \\
8.9 \\
0.019 \\
26,472\end{array}$ & $\begin{array}{r}1,172.58 \\
0.0\end{array}$ & & \begin{tabular}{r|}
14,405 \\
140.71 \\
10.0 \\
0.044 \\
40,877
\end{tabular} & & & $\begin{array}{r}17,261 \\
871.94 \\
11.1 \\
0.083 \\
58,138\end{array}$ & & & $\begin{array}{r}14,125 \\
499.39 \\
8.5 \\
0.065 \\
72,263 \\
\end{array}$ & & & $\begin{array}{r}17,783 \\
898.21 \\
9.0 \\
0.076 \\
90,046 \\
\end{array}$ & & & $\begin{array}{r}16,681 \\
448.49 \\
12.1 \\
0.063 \\
106,730\end{array}$ & & \begin{tabular}{r|}
106,730 \\
$4,508.45$ \\
84.7 \\
0.082 \\
106,730 \\
\end{tabular} & \begin{tabular}{r|}
27.417 \\
$1,499.87$ \\
18.9 \\
0.089 \\
40.877 \\
\end{tabular} & \begin{tabular}{|r}
65.853 \\
$2,718.03$ \\
40.7 \\
0.072 \\
106,730
\end{tabular} \\
\hline
\end{tabular}

Truck 1505 LNG

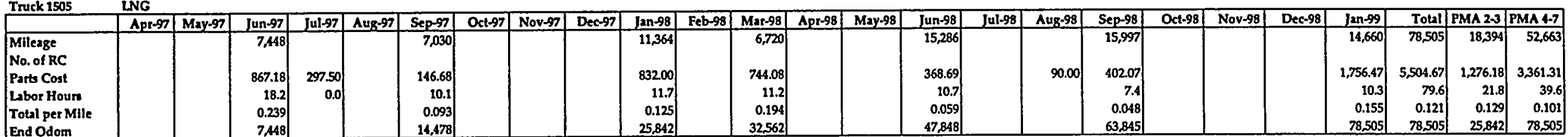

Truck 1506 LNG

\begin{tabular}{|c|c|c|c|c|c|c|c|c|c|c|c|c|c|c|c|c|c|c|c|c|c|c|c|c|c|}
\hline & Apr-97 & May-97 & Jun-97| & Jul-97| & Aug-97 & Sep-97! & Oct.97| & Nov-97 & Dec-97 & Jan-98 & Feb-98 & Mat-98 & Apr-98 & May-98 & \begin{tabular}{|c|} 
Jun-98 \\
\end{tabular} & Jul-98 & Aug-98 & Sep-98 & Oct-98 & Nov-98 & Dec-98 & Jan-99 & Total & PMA 2-3 & \begin{tabular}{|l|} 
PMA 4-7 \\
\end{tabular} \\
\hline $\begin{array}{l}\text { Milleage } \\
\text { No. of RC } \\
\text { Parts Cost } \\
\text { Labor Hours } \\
\text { Total per Milte } \\
\text { End Odom }\end{array}$ & & & & \begin{tabular}{r|}
11,753 \\
240.45 \\
20.4 \\
0.107 \\
11,753
\end{tabular} & & $\begin{array}{r}229.87 \\
0.0\end{array}$ & \begin{tabular}{r|}
13,503 \\
197.01 \\
7.4 \\
0.042 \\
25,256
\end{tabular} & & \begin{tabular}{r|r|}
10,212 & \\
144.38 & \\
10.9 & \\
0.068 & \\
35,468 &
\end{tabular} & & & \begin{tabular}{r|}
14,421 \\
136.58 \\
5.1 \\
0.027 \\
49,889
\end{tabular} & & & \begin{tabular}{r|}
15,115 \\
\\
$1,137.72$ \\
9.7 \\
0.107 \\
65,004
\end{tabular} & & 510.00 & 783.98| & $\begin{array}{r}11,400 \\
595.53 \\
13.9 \\
0.113 \\
76,404 \\
\end{array}$ & & & \begin{tabular}{r|}
13,270 \\
$1,240.59$ \\
11.4 \\
0.136 \\
89,674
\end{tabular} & \begin{tabular}{r|}
89,674 \\
$5,216.11$ \\
78.8 \\
0.102 \\
89,674
\end{tabular} & \begin{tabular}{|r|}
23,715 \\
\\
571.26 \\
18.3 \\
0.063 \\
35,468 \\
\end{tabular} & \begin{tabular}{|r|}
54,206 \\
$4,404.40$ \\
40.1 \\
0.118 \\
89,674 \\
\end{tabular} \\
\hline
\end{tabular}

Truck 1507 LNG

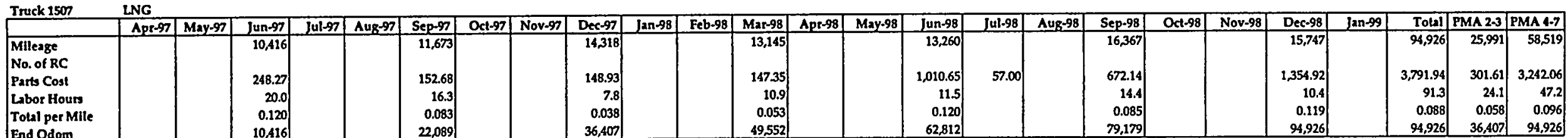

Truck 1508 ING

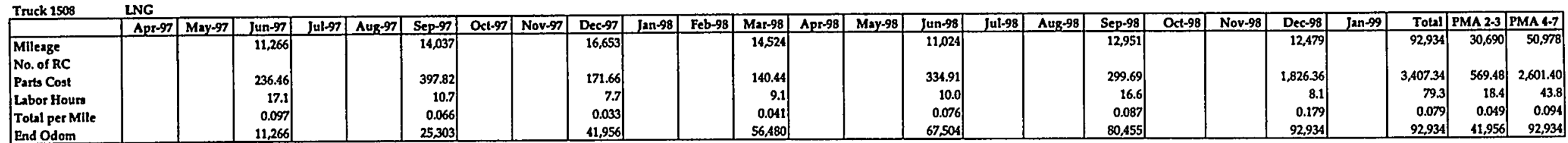

(a) $M$

25,303

$\begin{array}{r}0.041 \\ 56,480 \\ \hline\end{array}$

80,455 
Driver Complaint Analysis

July 1998 Through December 31, 1998

Raley's Diesel Study Fleet

$4 / 22 / 99$

\begin{tabular}{|c|c|c|c|c|c|c|c|c|}
\hline Category & Jul & Aug & Sep & Oct & Nov & Dec & Totals & Percent \\
\hline Body and Damage & 0 & $\overline{0}$ & $\overline{1}$ & $\overline{2}$ & 2 & $\overline{1}$ & $\overline{8}$ & 21 \\
\hline Lights & 2 & 2 & 2 & 2 & 2 & 0 & 10 & 26 \\
\hline Engine - Rough, Power & 0 & 0 & 0 & 0 & c & 0 & 0 & 0 \\
\hline Engine - Runs Better & 0 & 0 & 0 & 0 & c & 0 & 0 & 0 \\
\hline Low Fuel Pressure Light & 0 & 0 & 0 & 0 & 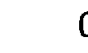 & 0 & 0 & 0 \\
\hline Fuel Leak & 0 & 0 & 0 & 0 & $\mathrm{c}$ & 0 & 0 & 0 \\
\hline Fuel Gauge & 0 & 0 & 0 & 0 & c & 0 & 0 & 0 \\
\hline Air/Suspension & 0 & 0 & 1 & 0 & 1 & 1 & 3 & 8 \\
\hline $\mathrm{AC} /$ Heat & 1 & 0 & 0 & 0 & c & 0 & 1 & 3 \\
\hline CB/Radio/Phone/Antenna & 0 & 2 & 0 & 0 & c & 1 & 3 & 8 \\
\hline Fifth Wheel & 0 & 0 & 0 & c & C & 0 & 0 & 0 \\
\hline Cruise Control & 0 & 0 & 0 & $\mathrm{c}$ & c & 6 & 6 & 16 \\
\hline Wheels & 0 & 0 & 0 & c & c & 0 & 0 & 0 \\
\hline Clutch & 0 & 0 & 0 & c & 1 & 0 & 1 & 3 \\
\hline CADEC & 0 & 0 & 0 & c & c & 0 & 0 & 0 \\
\hline Starter/Alternator/Battery & 0 & 0 & 1 & $c$ & c & 0 & 1 & 3 \\
\hline Tire & 1 & 0 & 0 & 2 & c & 0 & 3 & 8 \\
\hline Other & 0 & 2 & 0 & c & c & 0 & 2 & 5 \\
\hline $\begin{array}{r}\text { Total } \\
\end{array}$ & 4 & 6 & 5 & 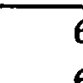 & $\varepsilon$ & 9 & 38 & 100 \\
\hline
\end{tabular}


Driver Complaint Analysis

April, 1997 Through December 31, 1998

Raley's LNG Fleet

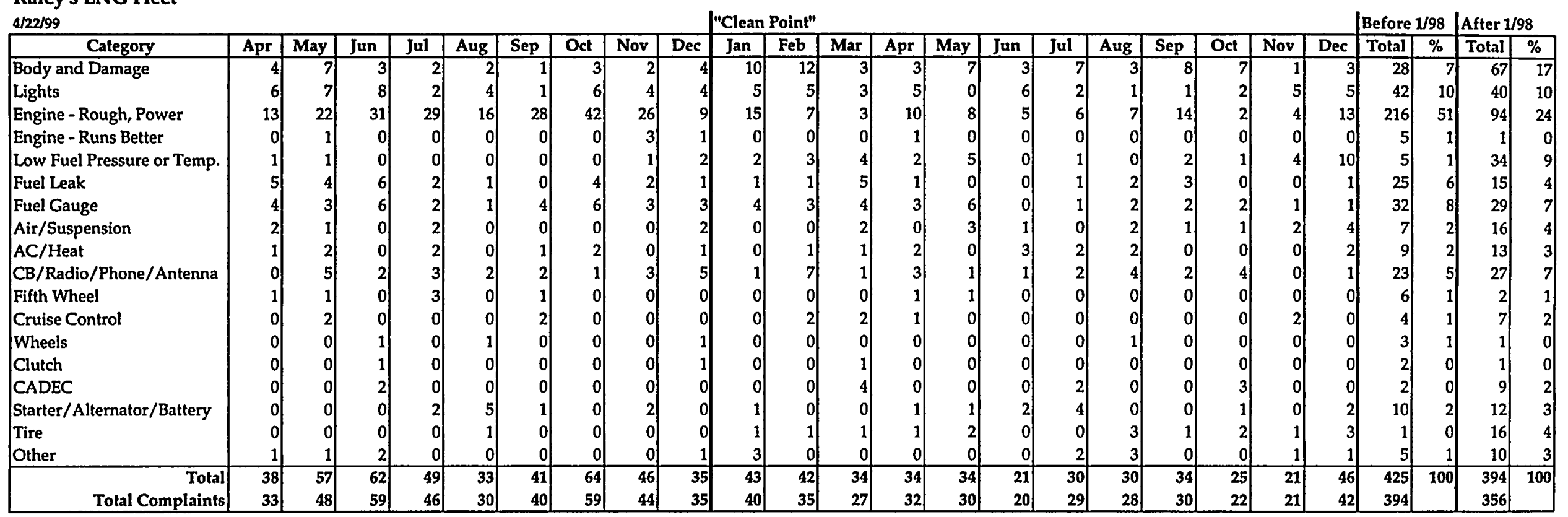




\section{Appendix $\mathrm{H}$}

\section{Emissions Testing Results}




\title{
WEST VIRGINIA UNIVERSITY Transportable Heavy Duty Vehicle Emissions Testing Laboratory
}

\section{Exhaust Emissions Test Results Report of Raley's Distribution Center Tractors}

\author{
Submitted to \\ Raley's Distribution Center
}

Department of Mechanical \& Aerospace Engineering

West Virginia University

Morgantown, WV 26506-6101

Phone: (304) 293-3111

September 30, 1997 


\section{Contents}

1 Executive Summary

2 Test Circumstance and Characteristics

2.1 Test Facility

2.2 Test Vehicles

2.3 Cycle

2.4 Test Procedure

3 Test Results

3.1 Summary

3.2 Short Report

3.3 Emission Comparison

3.4 Comments on Results 


\section{Executive Summary}

In order to collect emissions data from diesel trucks and compare them with their liquefied natural gas (LNG) sister trucks in Sacramento, CA, the West Virginia University (WVU) Transportable Heavy Duty Vehicle Emissions Testing Laboratory conducted a series of emissions tests on four diesel trucks and eight LNG trucks in the summer of 1997, with the cooperation of the Raley's Distribution Center (RDC) in Sacramento, CA. All RDC diesel trucks were powered with Cummins M11-280E engines and LNG trucks were powered with Cummins L10-300G engines. All vehicles were tested in the condition that they were received without any engine adjustments before testing.

Emissions data were acquired using the Five-Mile Route. This cycle was used to test all trucks for emissions comparison with similar trucks. The Five-Mile Route allows free (Maximum) acceleration during each of its five acceleration ramps. Time to complete the route may vary from vehicle type to vehicle type, but the target distance remains at 5 miles. Braking rates are prescribed, not variable. Repeat test-runs were conducted for each cycle with a 20-minute soak between each run.

Test results include both gaseous and particulate matter (PM) emissions as well as fuel economy inferred from emissions measurements. The emissions data, such as carbon monoxide ( $\mathrm{CO}$ ), carbon dioxide $\left(\mathrm{CO}_{2}\right)$, oxides of nitrogen $\left(\mathrm{NO}_{\mathrm{x}}\right)$, and total hydrocarbons $(\mathrm{HC})$, were calculated based on the continuous measurement during a test cycle. Samples of the dilute exhaust were shipped to the Gas Chromatograph laboratory at West Virginia University to obtain the measurement of the non-methane hydrocarbons (NMHC). The PM emissions were measured gravimetrically using a secondary dilution filter $(70 \mathrm{~mm})$ sampling of the primary diluted exhaust. Fuel economy was determined by carbon-balance method based upon the weight of carbon contained in exhaust constituents. Emissions results were reported in grams/mile whereas fuel economy was presented in miles/gallon and BTU/mile. For LNG fueled vehicles, MPG means miles per equivalent gallon diesel, or 137 cubic feet CNG at standard temperature and pressure is equivalent to 1 gallon of \#1 diesel.

Data quality control methods, such as evaluation of continuous data, two-person weighing of particulate filters, and monitoring of individual test results for abnormalities were used throughout the program to assure the reliability of the test emissions data. In each case, variation between repeat runs was noted. 


\section{Test Circumstances and Characteristics}

\subsection{Test Facility}

The WVU Transportable Heavy Duty Vehicle Emissions Testing Laboratory (THDVETL) is a chassis dynamometer testing facility that measures exhaust emissions from heavy-duty vehicles, such as transit buses, refuse trucks, and class 8 trucks and tractors. In the past five years, the two WVU transportable emissions testing laboratories have traveled from coast to coast and conducted over 900 tests on heavy duty vehicles and transit buses for 36 fleet owners in 17 states. Details on prior emissions measurements and the alternative fuels program may be found in the following papers: SAE 961082 , SAE 951016, and SAE 952746.

\subsection{Test Vehicles}

A total of twelve tractors was tested in this study. All diesel vehicles were powered with Cummins M1 1-280E engines, and all LNG vehicles were powered with Cummins L10-300G engines. The diesel vehicles had older vehicle and engine model years and higher mileage accumulation than the LNG vehicles. The diesel vehicles had splitter transmissions while the LNG vehicles had ranger transmissions.

\subsection{Cycle}

All vehicles were tested using the Five-Mile Route. This cycle was a modification of WVU FivePeak truck cycle that was originally described in SAE papers 941946 and 951016 . The Five-Mile Route is similar to the original WVU Five-Peak truck cycle that has five steady speeds, while it differs from Five-Peak cycle when it accelerates to the steady speed using the highest possible acceleration for each peak speed. To make test results comparable to each other, the driving distance for both of these cycles is held at five miles and the driving duration including idle time is held at 900.1 seconds. A sample actual driving speed for LNG tractor 1501 is shown in figure 1.

Figure 1. Sample Actual Driving Speed for Tractor 1501 (LNG Fuel, 5-Mile Route)

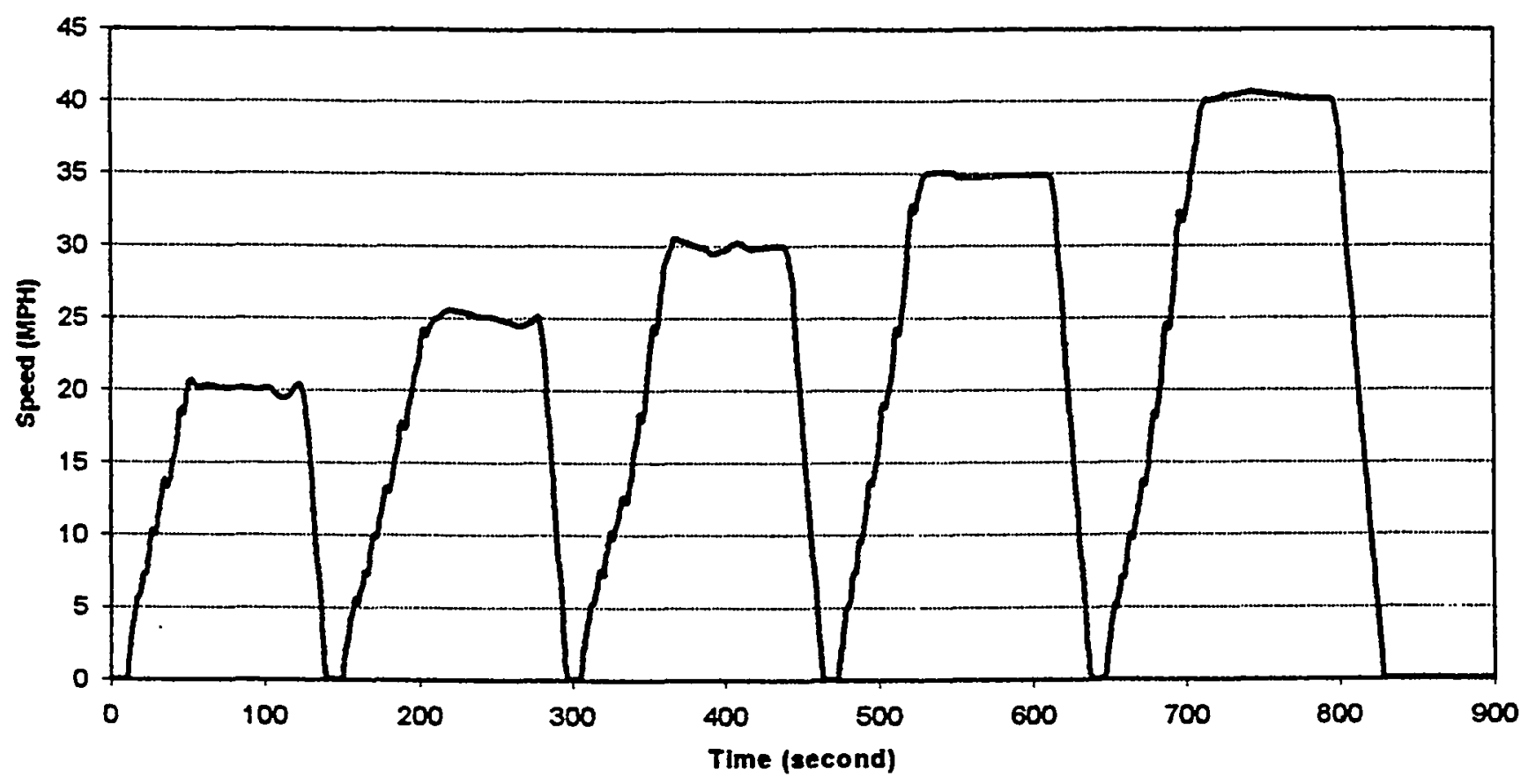




\subsection{Test Procedure}

Test procedure included calibration of equipment, vehicle inspection, actual vehicle testing, data reduction, and data analysis.

In order to make sure that the analyzers and the associated systems were functioning properly and providing measurements accurately, all instruments were calibrated or adjusted daily. Propane injections confirmed reliability of the dilution tunnel system.

Prior to the actual testing, all gearboxes in the power train of the dynamometer were warmed up to minimize loading variability due to the viscosity of the oil. Each test includes several repeat test-runs in order to guarantee that the exhaust emissions measured are a true representation of the test vehicle. The emissions data reported for each vehicle test are the average values of at least three repeat test-runs. All test runs were executed with the vehicle at operating temperature (no cold-start tests were performed).

The following procedures were adopted for the actual vehicle testing:

1) The vehicle was inspected prior to testing;

2) The vehicle was positioned and mounted on the chassis dynamometer

3) The vehicle was driven while on the test bed to warm-up the gearboxes of the power train of the dynamometer until they reached $100^{\circ} \mathrm{F}$, also ensuring that the vehicle's engine is at operational temperature before testing.

4) A practice test cycle was performed to allow the driver to become familiar with vehicle characteristics and to insure proper range settings of analyzers;

5) After completion of the practice run or one test run, the vehicle transmission was set to neutral and the engine was left in this condition for 17 minutes into a 20 minute soak period;

6) At 17 minutes into the soak period, the vehicle was driven though three of the CBD ramps to expel collected constituents in the exhaust system. Twenty seconds after completion of the final practice ramp, data collection was initiated.

7) 5) and 6) were repeated at least two times.

All emissions data, except for PM and non-methane hydrocarbons, were obtained by continuous readings and the results were integrated over the complete test cycle. PM emissions were measured gravimetrically using a secondary dilution filter sampling of the primary diluted exhaust. Non-methane hydrocarbons were measured by a gas chromatograph (GC) laboratory for all the CNG vehicles. GC results were used to determine the methane/non-methane split, which was applied to the HC data measured in the field. Differing responses of the field instrument to methane and propane were taken into account.

During a test, the driver was provided with a visual trace of the scheduled speed versus time on an interface monitor. The speed trace was a representation of the test cycle being used. The driver followed the speed trace closely to minimize the errors introduced by irregular driving.

All of the vehicles were jacked up with hydraulic jacks placed on scales until the rear tires were no longer on the rollers of the test bed. The vehicle was then lowered onto the rollers until the vehicle was centered on the test bed. 


\section{Test Results}

This section includes all the test results and their comparison from four diesel trucks and eight LNG trucks using the Five-Mile Route.

\subsection{Summary}

Test results have been presented in three formats: short report, emissions data in grams/mile, and emissions data summary and comparison based on different fuel.

\subsection{Short Report}

The short report shows the vehicle information, vehicle engine information, emissions data in grams/mile, and fuel economy for each test run, average emissions over all test runs, and brief comments for each test in a compact format on one page. The odometer mileage reading in the short reports is rounded to the nearest 100 miles.

Symbols used in Short Report Emissions data result table:

a A value was measured and identified as an apparent outliar, and therefore is not reported and not used to compute other parameters or the average values.

b The residual hydrocarbon emissions (RHC) is calculated from the difference between the methanol $\left(\mathrm{CH}_{3} \mathrm{OH}\right)$ and the FID-HC concentrations. For $100 \%$ alcohol fuels, the value of RHC is small and due to experimental variations, it may be measured as positive or negative but can best be assumed to be zero.

c A value cannot be calculated because the parameters required for calculation are not available.

d A value of coefficient variance (CV\%) is not meaningful because the average value is too small or not available. A significant coefficient of variance may exist for PM from CNG vehicles, where the PM is at very low levels. Note that CNG PM is more than an order of magnitude less than PM usually measured from diesel vehicles. Similarly some modern diesel vehicles will yield very low hydrocarbon emissions.

All short reports for the Raley's Distribution Center trucks are included in this report.

\subsection{Emissions Comparison}

The emissions for these tests are compared with each other and other sister vehicles using the same fuels. Emissions units are in grams/mile. Data in the final emissions summary show that NOx was lower for the LNG trucks than the diesel trucks. LNG also showed significant advantage in PM reduction, but returned $\mathrm{CO}$ levels higher than those for diesel. Also the energy-based equivalent fuel consumption of the LNG trucks was about $28.7 \%$ lower than the diesel trucks. Although LNG total hydrocarbon emissions were higher than for diesel, only about $5 \%$ of the CNG hydrocarbon emissions were non-methane organic gases.

\subsection{Comments on Results}

The LNG vehicles returned NOx emissions that were six times lower than those from the diesel trucks. Emissions of NOx from one of the LNG vehicles were distinctly higher than from the remainder: $\mathrm{HC}$ for this vehicle was the lowest, and so it is assumed that this vehicle was operating in a less lean fashion than the other vehicles. PM for the LNG vehicles was at one tenth that of the diesel vehicles, and NMHC for the LNG vehicles was at half of the HC level for the diesel vehicles. 
Test Sequence Number: 950

WVU Test Reference Number: WVU-TEDDY2-D2-5MILES

Fleet Owner Full Name

Fleet Address

Fleet Address (City, State, Zip)

Vehicle Type

Vehicle ID Number (VIN)

Vehicle Manufacturer

Vehicle Model Year

Gross Vehicle Weight (GWM) (b.)

Vehicle Total Curb Weight (lb.)

Vehicle Tested Weight (b.)

Odometer Reading (mile)

Transmission Type

Transmission Configuration

Number of Axles

Engine Type

Engine ID Number

Engine Displacement (Liter)

Number of Cylinders

Engine Rated Power (hp)

Primary Fuel

Test Cycle

Test Date

Engineer

Driver
West Virginia University

MAE Dept., P. O. Box 6106

Morgantown, WV 26506

Tractor Truck

1FUPYSYB7FH258124

Freightliner

1985

80000

12000

42000

740100

Manual

9-Speed

3

Caterpillar $3406 B$

7FB30060

14

6

350

D2

5 Mile Route

7/8/97

J. Boyce

L. MCGrath

Emissions Results (g/mile)

Fuel Economy

\begin{tabular}{|c|c|c|c|c|c|c|c|c|}
\hline Run Seq.NO- & $\mathrm{CO}$ & $\mathrm{NO}_{\mathrm{x}}$ & $\mathrm{FIDHC}$ & $\mathrm{PM}$ & $=\mathrm{CO}_{2}$ & mile/gal & BTU/mile- & Miles - \\
\hline $950-3$ & 7.64 & 39.2 & 2.16 & 1.33 & 1849 & 5.45 & 23857 & 5.00 \\
\hline $950-4$ & 8.97 & 39.0 & 1.95 & 1.29 & 1825 & 5.52 & 23571 & 4.95 \\
\hline $950-5$ & 7.84 & 39.4 & 2.52 & 1.44 & 1833 & 5.49 & 23676 & 4.99 \\
\hline $950-6$ & 7.03 & 39.4 & 2.02 & 1.22 & 1824 & 5.52 & 23531 & 5.00 \\
\hline & & & & & & & & \\
\hline 950 Average & 7.87 & 39.2 & 2.16 & 1.32 & 1833 & 5.49 & 23659 & 4.98 \\
\hline Std. Dov. & 0.81 & 0.2 & 0.25 & 0.09 & 11 & 0.03 & 146 & 0.03 \\
\hline CV\% & 10.3 & 0.5 & 11.7 & 7.0 & 0.6 & 0.6 & 0.6 & 0.5 \\
\hline
\end{tabular}

Test Purpose:

Measurement of exhaust constituents from WVU Transportable Heavy Duty Emissions Laboratory-2 control vehicle for comparison of emissions on the same vehicle at different locations. 


\section{Test Sequence Number: 968}

\section{WVU Test Reference Number: RDC-1504-LNG-5MILES}

Fleet Owner Full Name

Fleet Address

Fleet Address (City, State, Zip)

Vehicle Type

Vehicle ID Number (VIN)

Vehicle Manufacturer

Vehicle Model Year

Gross Vehicle Weight (GWW) (bb.)

Vehicle Total Curb Weight (Ib.)

Vehicle Tested Weight (lb.)

Odometer Reading (mile)

Transmission Type

Transmission Configuration

Number of Axdes

Engine Type

Engine ID Number

Engine Displacement (Liter)

Number of Cylinders

Engine Rated Power (hp)

Primary Fuel

Test Cycle

Test Date

Engineer

Driver

\author{
Raley's Distribution Center \\ 4061 Gateway Park Blvd. \\ Sacramento, CA 95834 \\ Tractor Truck \\ 1XKDD59X6VR745376 \\ Kenworth \\ 1997 \\ 48000 \\ 16540 \\ 42000 \\ 17000 \\ Manual \\ 10-Speed \\ 3 \\ Cummins L10-300G \\ 34847352 \\ 10 \\ 6 \\ 300 \\ LNG \\ 5 Mile Route \\ 7/25/97 \\ S. McConnell \\ L. MOGrath
}

Emissions Results ( $\mathrm{g} / \mathrm{mile}$ )

\begin{tabular}{|c|c|c|c|c|c|c|c|c|c|c|}
\hline Run Seg. No. & CO & NO $_{x}$ & FIDHC & CH $_{4}$ & NMHC & PM & $C_{2}$ & mile/gal & BTU/mile & Milles \\
\hline $968-1$ & 7.17 & 13.2 & 15.3 & 13.50 & 0.52 & 0.069 & 1647 & 4.42 & 29072 & 5.00 \\
\hline $968-2$ & 8.42 & 12.9 & 15.5 & $C$ & $C$ & 0.070 & 1631 & 4.46 & 28840 & 5.00 \\
\hline $968-3$ & 8.04 & 11.2 & 15.4 & 13.59 & 0.54 & 0.069 & 1620 & 4.49 & 28644 & 5.01 \\
\hline $968-4$ & 7.36 & 12.4 & 14.5 & 12.69 & 0.62 & 0.053 & 1562 & 4.66 & 27587 & 5.00 \\
\hline $968-5$ & 7.62 & 15.6 & 14.8 & $c$ & $c$ & 0.057 & 1682 & 4.33 & 29653 & 5.01 \\
\hline $968-6$ & 7.67 & 11.6 & 14.2 & 12.41 & 0.59 & 0.053 & 1645 & 4.43 & 28997 & 5.00 \\
\hline & & & & & & & & & & \\
\hline 968 Average & 7.74 & 12.8 & 15.0 & 13.05 & 0.57 & 0.064 & 1631 & 4.46 & 28799 & 5.00 \\
\hline Std. Dev. & 0.46 & 1.5 & 0.5 & 0.59 & 0.04 & 0.007 & 40 & 0.11 & 684 & 0.00 \\
\hline CV\% & 5.9 & 12.1 & 3.6 & 4.5 & 7.5 & 11.3 & 2.4 & 2.4 & 2.4 & 0.1 \\
\hline
\end{tabular}

\section{Test Purpose:}

Collection of emissions data from LNG fueled vehictes to establish a data set for use in comparison with diesel emissions.

\section{Special Procedures:}

Test program was modified to ensure that 5-Mile-Route finished five miles for each test run even though the road speed govemor kept the driver from shifting gears and the driver drove more slowiy than nomal. All tests running 5-Mile-Route in this test site since this one finished five miles for each test run. 
Test Sequence Number: 969

WWU Test Reference Number: RDC-1505-LNG-5MILES

Fleet Owner Full Name

Fleet Address

Fleet Address (City, State, Zip)

Vehicle Type

Vehicle ID Number (VIN)

Vehicle Manufacturer

Vehicle Model Year

Gross Vehicle Weight (GWW) (b.)

Vehicle Total Curb Weight (b.)

Vehicle Tested Weight (lb.)

Odometer Reading (mile)

Transmission Type

Transmission Configuration

Number of Axles

Engine Type

Engine ID Number

Engine Displacement (Liter)

Number of Cylinders

Engine Rated Power (hp)

Primary Fuel

Test Cycle

Test Date

Engineer

Driver
Raley's Distribution Center

4061 Gateway Park Bivd.

Sacramento, CA 95834

Tractor Truck

1XKDD59X8VR745377

Kenworth

1997

48000

15720

42000

10700

Manual

10-Speed

3

Cummins L10-300G

34849168

10

6

300

LNG

5 Mile Route

7/26/97

S. McConnell

L. MCGrath

Emissions Results (g/mile)

Fuel Economy

\begin{tabular}{|c|c|c|c|c|c|c|c|c|c|c|}
\hline Run Seg. No. & $\mathrm{CO}$ & NO $_{x}$ & FIDHC & $\mathrm{CH}_{4}$ & NMHC & PM & $\mathrm{CO}_{2}$ & mile/gal & BTU/mile & Milles \\
\hline $969-1$ & 7.53 & 4.33 & 20.6 & 18.21 & 0.66 & 0.101 & 1706 & 4.24 & 30342 & 5.01 \\
\hline $969-2$ & 7.36 & 4.60 & 19.0 & 16.74 & 0.69 & 0.122 & 1692 & 4.28 & 30026 & 5.02 \\
\hline $969-3$ & 7.22 & 5.13 & 18.7 & 16.50 & 0.65 & 0.096 & 1702 & 4.26 & 30172 & 5.01 \\
\hline $969-4$ & 7.00 & 5.23 & 18.2 & 15.95 & 0.69 & 0.085 & 1716 & 4.23 & 30372 & 5.00 \\
\hline $969-5$ & 7.04 & 5.16 & 17.1 & 14.99 & 0.65 & 0.088 & 1722 & 4.22 & 30435 & 5.01 \\
\hline $969-6$ & 6.77 & 5.57 & 16.7 & 14.69 & 0.64 & 0.077 & 1644 & 4.42 & 29070 & 5.01 \\
\hline & & & & & & & & & & \\
\hline 968 Average & 7.16 & 5.00 & 18.4 & 16.18 & 0.66 & 0.095 & 1697 & 4.27 & 30069 & 5.01 \\
\hline Std. Dov. & 0.27 & 0.45 & 1.4 & 1.28 & 0.02 & 0.016 & 28 & 0.07 & 512 & 0.01 \\
\hline CV\% & 3.8 & 9.0 & 7.7 & 7.9 & 3.2 & 16.5 & 1.7 & 1.7 & 1.7 & 0.1 \\
\hline
\end{tabular}

\section{Tost Purpose:}

Collection of emissions data from LNG fueled vehicles to establish a data set for use in comparison with diesel emissions. 


\section{Test Sequence Number: 970}

WVU Test Reference Number: RDC-1506-LNG-5MILES

Fleet Owner Full Name

Fleet Address

Fleet Address (City, State, Zip)

Vehicie Type

Vehicle ID Number (MN)

Vehicle Manufacturer

Vehicle Model Year

Gross Vehicle Weight (GWM) (Ib.)

Vehicle Total Curb Weight (lb.)

Vehicle Tested Weight (lb.)

Odometer Reading (mile)

Transmission Type

Transmission Configuration

Number of Axles

Engine Type

Engine ID Number

Engine Displacement (Liter)

Number of Cylinders

Engine Rated Power (hp)

Primary Fuel

Test Cycle

Test Date

Engineer

Driver
Raley's Distribution Center

4061 Gateway Park Blvd.

Sacramento, CA 95834

Tractor Truck

1XKDD59XXVR745378

Kenworth

1997

48000

16520

42000

15000

Manual

10-Speed

3

Cummins L10-300G

34848166

10

6

300

LNG

5 Mile Route

7/28/97

S. McConnell

L. MoGrath

Emissions Results (g/mile)

Fuel Economy

\begin{tabular}{|c|c|c|c|c|c|c|c|c|c|c|}
\hline Run Seq. No. & $\mathrm{CO}$ & $\mathrm{NO}_{\mathrm{x}}$ & FIDHC & $\mathrm{CH}$. & NMHC & PM & $\mathrm{CO}_{2}$ & mile/gal & BTU/mile & Miles \\
\hline $970-1$ & 6.93 & 4.45 & 15.4 & 13.53 & 0.56 & 0.048 & 1669 & 4.36 & 29444 & 4.99 \\
\hline $970-2$ & 6.88 & 4.56 & 16.5 & 14.54 & 0.58 & 0.048 & 1654 & 4.40 & 29239 & 5.00 \\
\hline $970-3$ & 6.77 & 4.34 & 15.2 & 13.35 & 0.55 & 0.060 & 1596 & 4.56 & 28188 & 5.00 \\
\hline $970-4$ & 6.70 & 4.66 & 14.8 & 13.00 & 0.57 & 0.052 & 1589 & 4.58 & 28046 & 5.00 \\
\hline & & & & & & & & & & \\
\hline 970 Average & 6.82 & 4.50 & 15.5 & 13.61 & 0.56 & 0.052 & 1627 & 4.48 & 28729 & 5.00 \\
\hline Std. Dev. & 0.11 & 0.14 & 0.7 & 0.66 & 0.01 & 0.006 & 40 & 0.11 & 714 & 0.01 \\
\hline CV\% & 1.6 & 3.1 & 4.7 & 4.9 & 2.0 & 10.9 & 2.5 & 2.5 & 2.5 & 0.2 \\
\hline
\end{tabular}

\section{Test Purpose:}

Collection of emissions data from LNG fueled vehicles to establish a data set for use in comparison with diesel emissions.

\section{Special Procedures:}

$\mathrm{HC}$ probe heater was found failed during the soak period between test \#2 and \#3. It was replaced with a new one and retumed back to nomal functioning. A practice test was run with no data taken to keep the test procedure followed.

\section{Observations:}

Driver found the vehicle was a little slower than other sister LNG tractors while it was smoother to shift gears than all others were. 


\section{Test Sequence Number: 971}

WVU Test Reference Number: RDC-1507-LNG-5MILES

Fleet Owner Full Name

Fleet Address

Fleet Address (City, State, Zip)

Vehicle Type

Vehicle ID Number (VIN)

Vehicle Manufacturer

Vehicle Model Year

Gross Vehicle Weight (GWM) (b.)

Vehicle Total Curb Weight (Ib.)

Vehicle Tested Weight (Ib.)

Odometer Reading (mile)

Transmission Type

Transmission Configuration

Number of Axles

Engine Type

Engine ID Number

Engine Displacement (Liter)

Number of Cylinders

Engine Rated Power (hp)

Primary Fuel

Test Cycle

Test Datè

Engineer

Driver
Raley's Distribution Center

4061 Gateway Park Blvd.

Sacramento, CA 95834

Tractor Truck
1XKDD59X1VR745379
Kenworth
1997
48000
15585
42000
14600
Manual
$10-$ Speed
3
Cummins L10-300G
34848167
10
6
300
LNG
5 Mile Route
$7 / 29 / 97$
S. MCConnell
L. MCGrath

Emissions Results (g/mile)

Fuel Economy

\begin{tabular}{|c|c|c|c|c|c|c|c|c|c|c|}
\hline Run Seg. NO: & $\mathrm{CO}$ & $\mathrm{NO}_{x}$ & FIDHC & $\mathrm{CH}_{4}$ & NMHC & PM & $\mathrm{CO}_{2}$ & mile/gal & BTU/mile & Miles \\
\hline $971-4$ & 6.49 & $\mathrm{a}$ & 18.8 & 16.54 & 0.63 & 0.083 & 1668 & 4.35 & 29569 & 5.01 \\
\hline $971-2$ & 6.59 & 3.66 & 17.9 & 15.80 & 0.59 & 0.069 & 1709 & 4.25 & 30241 & 5.01 \\
\hline $971-3$ & 6.56 & 3.81 & 18.6 & 16.39 & 0.60 & 0.080 & 1758 & 4.13 & 31095 & 5.02 \\
\hline $971-4$ & 6.66 & 3.72 & 18.7 & 16.46 & 0.67 & 0.059 & 1761 & 4.12 & 31163 & 5.00 \\
\hline $971-5$ & 6.57 & 3.09 & 18.0 & 15.81 & 0.63 & 0.064 & 1665 & 4.36 & 29483 & 5.01 \\
\hline $971-6$ & 6.64 & 3.98 & 17.9 & 15.79 & 0.62 & 0.071 & 1639 & 4.42 & 29049 & 5.01 \\
\hline & & & & & & & & & & \\
\hline 971 Average & 6.58 & 3.65 & 18.3 & 16.13 & 0.62 & 0.071 & 1700 & 4.27 & 30100 & 5.01 \\
\hline Std. Dev. & 0.06 & 0.34 & 0.4 & 0.37 & 0.03 & 0.009 & 51 & 0.12 & 884 & 0.00 \\
\hline CV\% & 0.9 & 9.21 & 2.3 & 2.3 & 4.8 & 12.8 & 3.0 & 2.9 & 2.9 & 0.1 \\
\hline
\end{tabular}

\section{Test Purpose:}

Collection of emissions data from LNG fueled vehicles to establish a data set for use in comparison with diesel emissions. 


\section{Test Sequence Number: 972}

\section{WVU Test Reference Number: RDC-1508-LNG-5MILES}

Fleet Owner Full Name

Fleet Address

Fleet Address (City, State, Zip)

Vehicle Type

Vehicle ID Number (VIN)

Vehicle Manufacturer

Vehicle Model Year

Gross Vehicle Weight (GWY) (bb.)

Vehicle Total Curb Weight (Ib.)

Vehicle Tested Weight (lb.)

Odometer Reading (mile)

Transmission Type

Transmission Configuration

Number of Axies

Engine Type

Engine ID Number

Engine Displacement (Liter)

Number of Cylinders

Engine Rated Power (hp)

Primary Fuel

Test Cycle

Test Date

Engineer

Driver

\author{
Raley's Distribution Center \\ 4081 Gateway Park Btvd. \\ Sacramento, CA 95834 \\ Tractor Truck \\ 1XKDD59X8VR745380 \\ Kenworth \\ 1997 \\ 48000 \\ 15880 \\ 42000 \\ 16300 \\ Manual \\ 10-Speed \\ 3 \\ Cummins L10-300G \\ 34849165 \\ 10 \\ 6 \\ 300 \\ LNG \\ 5 Mile Route \\ 7/30/87 \\ J. Kopasko \\ L. MoGrath
}

Emissions Results (g/mile)

Fuel Economy

\begin{tabular}{|c|c|c|c|c|c|c|c|c|c|c|}
\hline Run Seq: No. & $\mathrm{CO}$ & $\mathrm{NO}_{x}$ & FIDHC & $\mathrm{CH}_{4}$ & $\mathrm{NMHC}$ & $\mathrm{PM}$ & $\mathrm{CO}_{2}$ & mile/gal & BTU/mile & Miles \\
\hline $972-1$ & 6.59 & 3.50 & 18.7 & 16.54 & 0.61 & 0.113 & 1740 & 4.17 & 30809 & 5.01 \\
\hline $972-2$ & 6.46 & 3.71 & 19.1 & 16.83 & 0.63 & 0.091 & 1766 & 4.11 & 31267 & 5.02 \\
\hline $972-3$ & 6.68 & 3.95 & 18.5 & 16.29 & 0.64 & 0.083 & 1832 & 3.97 & 32366 & 5.01 \\
\hline $972-4$ & $\mathrm{C}$ & 4.17 & 18.3 & 16.09 & 0.67 & 0.086 & 1788 & 4.07 & 31552 & 5.02 \\
\hline $972-5$ & 6.54 & 3.31 & 18.9 & 16.66 & 0.66 & 0.098 & 1726 & 4.20 & 30580 & 5.01 \\
\hline $972-6$ & 6.75 & 4.11 & 18.3 & 16.09 & 0.67 & 0.081 & 1738 & 4.18 & 30759 & 5.02 \\
\hline & & & & & & & & & & \\
\hline 972 Average & 6.60 & 3.79 & 18.6 & 16.42 & 0.64 & 0.092 & 1765 & 4.12 & 31222 & 5.01 \\
\hline Std. Dev. & 0.11 & 0.34 & 0.3 & 0.31 & 0.03 & 0.012 & 40 & 0.09 & 660 & 0.01 \\
\hline CV\% & 1.7 & 9.1 & 1.7 & 1.9 & 3.9 & 13.0 & 2.2 & 2.1 & 2.1 & 0.1 \\
\hline
\end{tabular}

\section{Test Pumpose:}

Collection of emissions data from LNG fueled vehicles to establish a data set for use in comparison with diesel emissions. 


\section{Test Sequence Number: 973}

WVU Test Reference Number: RDC-1501-LNG-5MILES-R

Fleet Owner Full Name

Fleet Address

Fleet Address (City, State, Zip)

Vehicle Type

Vehicle ID Number (VIN)

Vehicle. Manufacturer

Vehicle Model Year

Gross Vehicle Weight (GWM) (bb.)

Vehicle Total Curb Weight (Ib.)

Vehicle Tested Weight (lb.)

Odometer Reading (mile)

Transmission Type

Transmission Configuration

Number of Axles

Engine Type

Engine ID Number

Engine Displacement (Liter)

Number of Cylinders

Engine Rated Power (hp)

Primary Fuel

Test Cycle

Test Date

\section{Engineer}

Driver
Raley's Distribution Center

4061 Gateway Park Blvd.

Sacramento, CA 95834

Tractor Truck

1XKDD59XOVR745373

Kenworth

1997

48000

16520

42000

13600

Manual

10-Speed

3

Cummins L10-300G

34845353

10

6

300

LNG

5 Mille Route

7/31/97

J. Kopasko

L. MoGrath

Emissions Results (g/mile)

\begin{tabular}{|c|c|c|c|c|c|c|c|c|c|c|}
\hline Run Seq. No. & $\mathrm{CO}$ & $\mathrm{NO}_{x}$ & FIDHC & CH4.. & NMHC & PM & $\mathrm{CO}_{2}$ & mile/gal & BTU/mile & Miles \\
\hline $973-1$ & 7.82 & $\mathrm{a}$ & 22.9 & 20.27 & 0.68 & 0.069 & 1722 & 4.18 & 30728 & 5.02 \\
\hline $973-2$ & 7.83 & 2.67 & 19.7 & 17.34 & 0.64 & 0.059 & 1707 & 4.24 & 30322 & 5.00 \\
\hline $973-3$ & 7.53 & 2.80 & 20.1 & 17.71 & 0.64 & 0.058 & 1719 & 4.29 & 30528 & 5.01 \\
\hline $973-4$ & 7.41 & 3.39 & 20.2 & 17.83 & 0.62 & 0.062 & 1661 & 4.35 & 29539 & 5.01 \\
\hline $973-5$ & 7.43 & 3.12 & 19.1 & 16.82 & 0.64 & 0.075 & 1659 & 4.36 & 29453 & 5.01 \\
\hline $973-6$ & $\mathrm{C}$ & 3.34 & 21.5 & 18.97 & 0.66 & 0.062 & 1656 & 4.35 & 29573 & 5.02 \\
\hline & & & & & & & & & & \\
\hline 973 Average & 7.00 & 3.06 & 20.5 & 18.19 & 0.65 & 0.064 & 1687 & 4.28 & 30024 & 5.01 \\
\hline Std. Dev. & 0.21 & 0.32 & 1.4 & 1.25 & 0.02 & 0.006 & 32 & 0.08 & 566 & 0.01 \\
\hline CV\% & 2.7 & 10.4 & 6.8 & 6.9 & 3.2 & 10.1 & 1.9 & 1.9 & 1.9 & 0.1 \\
\hline
\end{tabular}

\section{Test Purpose:}

Collection of emissions data from LNG fueled vehicles to establish a data set for use in comparison with diesel emissions.

\section{Observations:}

Driver found the vehicle was less powerful than other sister LNG tractors and surged during the test. 
Test Sequence Number: 974

WVU Test Reference Number: RDC-1502-LNG-5MILES-R

$\begin{array}{ll}\text { Fleet Owner Full Name } & \text { Raley's Distribution Center } \\ \text { Fleet Address } & 4061 \text { Gateway Park Blvd. } \\ \text { Fleet Address (City, State, Zip) } & \text { Sacramento, CA 95834 } \\ & \\ \text { Vehicle Type } & \text { Tractor Truck } \\ \text { Vehicle ID Number (VIN) } & \text { 1XKDD59X2VR745374 } \\ \text { Vehicle Manufacturer } & \text { Kenworth } \\ \text { Vehicle Model Year } & 1997 \\ \text { Gross Vehicle Weight (GWW (lb.) } & 48000 \\ \text { Vehicle Total Curb Weight (lb.) } & 16380 \\ \text { Vehicle Tested Weight (lb.) } & 42000 \\ \text { Odometer Reading (mile) } & 13900 \\ \text { Transmission Type } & \text { Manual } \\ \text { Transmission Configuration } & 10-5 p e e d \\ \text { Number of Axles } & 3 \\ & \\ \text { Engine Type } & \text { Cummins L10-300G } \\ \text { Engine ID Number } & 34847019 \\ \text { Engine Displacement (Liter) } & 10 \\ \text { Number of Cylinders } & 6 \\ \text { Engine Rated Power (hp) } & 300 \\ & \\ \text { Primary Fuel } & \text { LNG } \\ \text { Test Cycle } & 5 \text { Mile Route } \\ \text { Test Date } & 8 / 1 / 97 \\ \text { Engineer } & \\ \text { Driver } & \text { J. Kopasko } \\ \text { L. McGrath }\end{array}$

Emissions Results (g/mile)

\begin{tabular}{|c|c|c|c|c|c|c|c|c|c|c|}
\hline Run Seq. No. & $\mathrm{CO}:$ & NO & FIDHC & $\mathrm{CH}_{x}$ & NMHC & PM & $\mathrm{CO}_{2}$ & mile/gal & BTU/mile & Miles \\
\hline $974-1$ & 6.70 & 2.93 & 17.4 & 15.37 & 0.56 & 0.042 & 1601 & 4.53 & 28376 & 5.00 \\
\hline $974-2$ & 6.57 & 2.89 & 16.0 & 14.13 & 0.51 & 0.039 & 1614 & 4.50 & 28525 & 5.00 \\
\hline $974-3$ & 6.75 & 3.01 & 16.2 & 14.32 & 0.51 & 0.042 & 1559 & 4.66 & 27600 & 5.01 \\
\hline $974-4$ & 6.52 & 2.68 & 16.5 & 14.59 & 0.50 & 0.039 & 1577 & 4.60 & 27912 & 5.02 \\
\hline & & & & & & & & & & \\
\hline 974 Average & 6.64 & 2.88 & 16.5 & 14.60 & 0.52 & 0.041 & 1588 & 4.57 & 28103 & 5.01 \\
\hline Std. Dev. & 0.19 & 0.14 & 0.6 & 0.54 & 0.03 & 0.002 & 25 & 0.07 & 425 & 0.01 \\
\hline CV\% & 1.6 & 5.0 & 3.8 & 3.7 & 5.3 & 4.3 & 1.5 & 1.5 & 1.5 & 0.2 \\
\hline
\end{tabular}

\section{Test Purpose:}

Collection of emissions data from LNG fueled vehicles to establish a data set for use in comparison with diesel emissions.

\section{Special Procedures:}

New spark plugs were installed before the test. 


\section{Test Sequence Number: 975}

\section{WVU Test Reference Number: RDC-1503-LNG-5MILES-R}

Fleet Owner Full Name

Fleet Address

Fleet Address (Clty, State, Zip)

Vehicle Type

Vehicle ID Number (MN)

Vehicle Manufacturer

Vehicle Model Year

Gross Vehicle Weight (GWY) (bb.)

Vehicle Total Curb Weight (lb.)

Vehicle Tested Weight (lb.)

Odometer Reading (mile)

Transmission Type

Transmission Configuration

Number of Axles

Engine Type

Engine ID Number

Engine Displacement (Liter)

Number of Cylinders

Engine Rated Power (hp)

Primary Fuel

Test Cycle

Test Daté

Engineer

Drver
Raley's Distribution Center

4081 Gateway Park Blvd.

Sacramento, CA 95834

Tractor Truck

1XKDD59X4VR745375

Kenworth

1997

48000

16560

42000

16300

Manual

10-Speed

3

Cummins L10-300G

34847353

10

6 .

300

LNG

5 Mile Route

8/2/87

J. Kopasko

L. MoGrath

Emissions Results (g/mile)

Fuel Economy

\begin{tabular}{|c|c|c|c|c|c|c|c|c|c|c|}
\hline Run:Seq. NO: & $\mathrm{CO}$ & $\mathrm{NO}_{\mathrm{x}}$ & $\mathrm{FIDHC}$ & $\mathrm{CH}_{4}$ & $\mathrm{NMHC}$ & $\mathrm{PM}$ & $\mathrm{CO}_{2}$ & mile/gal & BTU/mile & Mlles \\
\hline $975-1$ & 7.03 & 4.21 & 17.6 & 15.49 & 0.61 & 0.064 & 1563 & 4.63 & 27734 & 5.01 \\
\hline $975-2$ & 8.10 & 4.96 & 18.1 & 16.04 & 0.55 & 0.052 & 1497 & 4.82 & 26660 & 5.02 \\
\hline $975-3$ & 6.60 & 5.33 & 16.0 & 14.04 & 0.57 & 0.055 & 1543 & 4.70 & 27314 & 5.01 \\
\hline $975-4$ & 8.61 & 5.64 & 17.2 & 15.18 & 0.58 & 0.060 & 1558 & 4.64 & 27684 & 5.00 \\
\hline $975-5$ & 8.45 & 6.67 & 17.0 & 15.03 & 0.54 & 0.054 & 1598 & 4.53 & 28349 & 5.02 \\
\hline $975-6$ & 8.16 & 6.62 & 17.4 & 15.31 & 0.59 & 0.055 & 1623 & 4.46 & 28791 & 5.00 \\
\hline & & & & & & & & & & \\
\hline 975 Average & 7.83 & 5.57 & 17.2 & 15.18 & 0.57 & 0.056 & 1564 & 4.63 & 27755 & 5.01 \\
\hline Std. Dev. & 0.82 & 0.96 & 0.7 & 0.66 & 0.03 & 0.004 & 44 & 0.13 & 752 & 0.01 \\
\hline CV\% & 10.4 & 17.2 & 4.2 & 4.4 & 4.7 & 7.6 & 2.8 & 2.7 & 2.7 & 0.2 \\
\hline
\end{tabular}

Test Purpose:

Collection of emissions data from LNG fueled vehicles to establish a data set for use in comparison with diesel emissions.

\section{Special Procedures:}

Vehicle tank delivery pressure was low previously. Delivery pressure was supposed to be 100 psi, but was only 70 psi.

Technician raised tank pressure to 110psi to ensure proper delivered pressure.

\section{Observations:}

Vehicle surged at the beginning of ramps causing $\mathrm{CO}$ emissions to be high. 
Test Sequence Number: 976

WVU Test Reference Number: RDC-1586-D2-5MILES-R2

Fleet Owner Full Name

Fleet Address

Fleet Address (City, State, Zip)

Vehicle Type

Vehicle ID Number (VIN)

Vehicie Manufacturer

Vehicle Model Year

Gross Vehicle Weight (GWM) (lb.)

Vehicle Total Curb Weight (lb.)

Vehicle Tested Weight (lb.)

Odometer Reading (mile)

Transmission Type

Transmission Configuration

Number of Axles

Engine Type

Engine ID Number

Engine Displacement (Liter)

Number of Cylinders

Engine Rated Power (hp)

Primary Fuel

Test Cycle

Test Date

Engineer

Driver
Raley's Distribution Center

4061 Gateway Park BNd.

Sacramento, CA 95834

Tractor Truck

1XKBD99X9TS690099

Kenworth

1995

50000

14440

42000

162700

Manual

10-Speed

3

Cummins M11-280E

34776206

10.8

6

280

D2

5 Mile Route

8/4/97

J. Kopasko

L. MoGrath

Emissions Results (g/mile)

\begin{tabular}{|c|c|c|c|c|c|c|c|c|}
\hline Run Seq. No & $\mathrm{CO}$ & $\mathrm{NO}_{x}$ & $\mathrm{FIDHC}$ & $\mathrm{PM}$ & $\mathrm{CO}_{2}$ & mile/gal & BTU/mile & Miles \\
\hline $976-2$ & 2.06 & 27.4 & 1.58 & 1.34 & 1527 & 6.63 & 19614 & 5.02 \\
\hline $976-3$ & 1.98 & 28.2 & 1.57 & 1.33 & 1513 & 6.68 & 19426 & 5.01 \\
\hline $976-4$ & 2.03 & 29.9 & 1.55 & 1.35 & 1560 & 6.49 & 20033 & 5.00 \\
\hline $976-5$ & 2.03 & 28.4 & 1.55 & 1.33 & 1592 & 6.36 & 20446 & 5.01 \\
\hline & & & & & & & & \\
\hline 976 Average & 2.02 & 28.5 & 1.56 & 1.34 & 1548 & 6.54 & 19880 & 5.01 \\
\hline Std. Dev. & 0.03 & 1.0 & 0.02 & 0.01 & 36 & 0.15 & 455 & 0.01 \\
\hline CV\% & 1.7 & 3.7 & 1.0 & 0.8 & 2.3 & 2.3 & 2.3 & 0.2 \\
\hline
\end{tabular}

\section{Test Purpose:}

Collection of emissions data from diesel fueled vehicles to establish a data set for use in comparison with LNG emissions. 


\section{Test Sequence Number: 977}

\section{WVU Test Reference Number: RDC-1593-D2-5MILES-R}

Fleet Owner Full Name

Fleet Address

Fleet Address (City, State, Zip)

Vehicle Type

Vehicle ID Number (VIN)

Vehicte Manufacturer

Vehicle Model Year

Gross Vehicle Weight (GWW) (b.)

Vehicle Total Curb Weight (lb.)

Vehicle Tested Weight (ib.)

Odometer Reading (mile)

Transmission Type

Transmission Configuration

Number of Axles

Engine Type

Engine ID Number

Engine Displacement (Liter)

Number of Cylinders

Engine Rated Power (hp)

Primary Fuel

Test Cycle

Test Date

Engineer

Driver

\author{
Raley's Distribution Center \\ 4061 Gateway Park BNd. \\ Sacramento, CA 95834 \\ Tractor Truck \\ 1XKBD99X4TS712784 \\ Kenworth \\ 1995 \\ 50000 \\ 14960 \\ 42000 \\ 168400 \\ Manual \\ 10-Speed \\ 3 \\ Cummins M11-280E \\ 34787997 \\ 10.8 \\ 6 \\ 280 \\ D2 \\ 5 Mile Route \\ 8/6/97 \\ J. Kopasko \\ L. McGrath
}

Emissions Results (g/mile)

\begin{tabular}{|c|c|c|c|c|c|c|c|c|}
\hline Run-Seq. No. & CO & NO $_{x}$ & FIDHC & PM & CO $_{2}$ & mile/gal & BTU/mile & Miles \\
\hline $977-1$ & 1.70 & 32.4 & 1.00 & 0.35 & 1608 & 6.30 & 20620 & 5.00 \\
\hline $977-2$ & 1.69 & 33.1 & 1.03 & 0.34 & 1636 & 6.20 & 20972 & 5.00 \\
\hline $977-3$ & 1.60 & 31.2 & 1.08 & 0.52 & 1633 & 6.21 & 20933 & 5.00 \\
\hline $977-4$ & 1.71 & 30.8 & 1.07 & 0.49 & 1619 & 6.26 & 20763 & 5.04 \\
\hline & & & & & & & & \\
\hline 977 Average & 1.67 & 31.8 & 1.04 & 0.42 & 1624 & 6.24 & 20822 & 5.00 \\
\hline Std. Dev. & 0.05 & 1.1 & 0.04 & 0.09 & 13 & 0.05 & 162 & 0.01 \\
\hline CV\% & 3.0 & 3.4 & 3.4 & 22.4 & 0.8 & 0.8 & 0.8 & 0.1 \\
\hline
\end{tabular}

Test Purpose:

Collection of emissions data from diesel fueled vehicles to establish a data set for use in comparison with LNG emissions.

\section{Special Procedures:}

Bees and nest were found in background bag suction tube. They were removed by tuming the switch on and off, and filter was replaced. 
Test Sequence Number: 978

WVU Test Reference Number: RDC-1592-D2-5MILES-R

Fleet Owner Full Name

Fleet Address

Fleet Address (City, State, Zip)

Vehicle Type

Vehicle ID Number (NIN)

Vehicle Manufacturer

Vehicle Model Year

Gross Vehicle Weight (GWM) (bb.)

Vehicle Total Curb Weight (lb.)

Vehicle Tested Weight (Ib.)

Odometer Reading (mile)

Transmission Type

Transmission Configuration

Number of Axdes

Engine Type

Engine ID Number

Engine Displacement (Liter)

Number of Cylinders

Engine Rated Power (hp)

Primary Fuel

Test Cycle

Test Date

Engineer

Driver
Raley's Distribution Center

4061 Gateway Park Bwd.

Sacramento, CA 95834

Tractor Truck

1XKBD99X2TS712783

Kenworth

1995

50000

14960

42000

132700

Manual

10-Speed

3

Cummins M11-280E

34786945

10.8

6

280

D2

5 Mile Route

$8 / 7 / 97$

J. Kopasko

L. McGrath

Emissions Results (g/mile)

\begin{tabular}{|c|c|c|c|c|c|c|c|c|}
\hline Run Seg. No. & $\mathrm{CO}$ & $\mathrm{NO}_{\mathrm{x}}$ & FIDHC & $\mathrm{PM}^{-}$ & CO & - mile/gal & BTU/mile & Miles \\
\hline $978-1$ & 1.91 & 33.6 & 1.26 & 0.76 & 1736 & 5.84 & 22267 & 5.01 \\
\hline $978-2$ & 2.01 & 34.3 & 1.27 & 0.73 & 1653 & 6.13 & 21213 & 5.01 \\
\hline $978-3$ & 1.97 & 35.9 & $\mathrm{C}$ & 0.76 & 1667 & 6.08 & 21386 & 5.02 \\
\hline $978-4$ & 1.94 & 36.9 & 1.32 & 0.75 & 1695 & 5.98 & 21744 & 5.00 \\
\hline & & & & & & & & \\
\hline 978 Average & 1.96 & 35.2 & 1.28 & 0.75 & 1688 & 6.01 & 21652 & 5.01 \\
\hline Std. Dev. & 0.04 & 1.5 & 0.03 & 0.01 & 36 & 0.13 & 465 & 0.01 \\
\hline CV\% & 2.2 & 4.2 & 2.1 & 1.5 & 2.2 & 2.1 & 2.1 & 0.2 \\
\hline
\end{tabular}

\section{Test Purpose:}

Collection of emissions data from diesel fueled vehicles to establish a data set for use in comparison with LNG emissions. 


\section{Test Sequence Number: 979}

WVU Test Reference Number: RDC-1579-D2-5MILES-R

Fleet Owner Full Name

Fleet Address

Fleet Address (City, State, Zip)

Vehicle Type

Vehicle ID Number (MIN)

Vehicle Manufacturer

Vehicle Model Year

Gross Vehicle Weight (GWM) (lb.)

Vehicle Total Curb Weight (lb.)

Vehicle Tested Weight (lb.)

Odometer Reading (mile)

Transmission Type

Transmission Configuration

Number of Axles

Engine Type

Engine ID Number

Engine Displacement (Liter)

Number of Cylinders

Engine Rated Power (hp)

Primary Fuel

Test Cycle

Test Date

Engineer

Driver
Raley's Distribution Center

4061 Gateway Park BNd.

Sacramento, CA 95834

Tractor Truck

1XKBD99X0SS643610

Kenworth

1994

50000

15100

42000

237800

Manual

10-Speed

3

Cummins M11-280E

34735917

10.8

6

280

D2

5 Mile Route

8/8/97

J. Kopasko

L. McGrath

Emissions Results (g/mile)

\begin{tabular}{|c|c|c|c|c|c|c|c|c|}
\hline Run Seq. No: & CO & NO & FIDHC & PM & :CO & mile/gal & BTU/mile & Milles \\
\hline $979-1$ & 2.09 & 34.0 & 1.27 & 0.41 & 1719 & 5.89 & 22058 & 5.00 \\
\hline $979-2$ & 2.12 & 33.7 & 1.22 & 0.41 & 1766 & 5.74 & 22650 & 5.00 \\
\hline $979-3$ & 2.11 & 32.6 & 1.23 & 0.39 & 1720 & 5.89 & 22065 & 5.01 \\
\hline $979-4$ & 2.20 & 33.8 & 1.36 & 0.40 & 1748 & 5.80 & 22432 & 4.99 \\
\hline & & & & & & & & \\
\hline 979 Average & 2.13 & 33.5 & 1.27 & 0.40 & 1738 & 5.83 & 22301 & 5.00 \\
\hline Std. Dev & 0.05 & 0.6 & 0.00 & 0.01 & 23 & 0.08 & 291 & 0.01 \\
\hline CV\% & 2.3 & 1.9 & 4.9 & 2.3 & 1.3 & 1.3 & 1.3 & 0.2 \\
\hline
\end{tabular}

\section{Test Purpose:}

Collection of emissions data from diesel fueled vehicles to establish a data set for use in comparison with LNG emissions. 


\section{Emissions Data Summary}

\begin{tabular}{|c|c|c|c|c|c|c|c|c|c|}
\hline Test ID & WVU Ref Num & Run ID & $\mathrm{CO}$ & NOX & HC & PM & $\mathrm{CO2}$ & CH4 & NMHC \\
\hline \multirow{9}{*}{971} & \multirow{6}{*}{ RDC-1507-LNG } & 1 & $\overline{6} .49$ & & 18.77 & 0.083 & $1 \overline{6 \overline{6} 8}$ & $16.5 \overline{4}$ & 0.63 \\
\hline & & 2 & 6.59 & 3.66 & 17.91 & 0.069 & 1709 & 15.80 & 0.59 \\
\hline & & 3 & 6.56 & 3.81 & 18.56 & 0.080 & 1758 & $16.39^{\circ}$ & 0.60 \\
\hline & & 4 & 6.66 & 3.72 & 18.72 & $0.059^{\circ}$ & $17 \ddot{61}$ & 16.46 & 0.67 \\
\hline & & 5 & 6.57 & 3.09 & 17.97 & 0.064 & 1665 & 15.81 & 0.63 \\
\hline & & 6 & 6.64 & 3.98 & 17.93 & 0.071 & 1639 & 15.78 & 0.62 \\
\hline & \multirow{3}{*}{\multicolumn{2}{|c|}{$\begin{array}{r}\text { Average: } \\
\text { Standard Deviation: } \\
\text { Coefficient of Variance: }\end{array}$}} & 6.58 & 3.65 & 18.31 & 0.071 & 1700 & 16.13 & 0.62 \\
\hline & & & 0.06 & 0.34 & 0.41 & 0.009 & 51 & 0.37 & 0.03 \\
\hline & & & $0.9 \%$ & $9.2 \%$ & $2.3 \%$ & $12.8 \%$ & $3.0 \%$ & $2.3 \%$ & $4.8 \%$ \\
\hline \multirow{10}{*}{972} & \multirow{6}{*}{ RDC-1508-LNG } & 1 & 6.59 & 3.50 & 18.73 & 0.113 & 1740 & 16.54 & 0.61 \\
\hline & & 2 & 6.46 & 3.79 & 19.07 & 0.091 & 1766 & 16.83 & 0.63 \\
\hline & & 3 & 6.68 & 3.95 & 18.60 & 0.083 & 1832 & $16.29^{\circ}$ & 0.64 \\
\hline & & 4 & & 4.17 & $18 . \overline{31}$ & 0.086 & 1788 & 16.09 & 0.67 \\
\hline & & 5 & 6.54 & 3.31 & 18.93 & 0.098 & 1726 & 16.66 & 0.68 \\
\hline & & 6 & 6.75 & 4.11 & 18.32 & 0.081 & 1738 & 16.09 & 0.67 \\
\hline & \multirow{3}{*}{\multicolumn{2}{|c|}{$\begin{array}{r}\text { Average: } \\
\text { Standard Deviation: } \\
\text { Coefficient of Variance: }\end{array}$}} & 6.60 & 3.79 & 18.64 & 0.092 & 1765 & 16.42 & 0.64 \\
\hline & & & 0.11 & 0.34 & 0.32 & 0.012 & 40 & 0.31 & 0.03 \\
\hline & & & $1.7 \%$ & $9.1 \%$ & $1.7 \%$ & $13.0 \%$ & $2.2 \%$ & $1.9 \%$ & $3.9 \%$ \\
\hline & \multirow{4}{*}{ RDC-1579-D2 } & 2 & 2.06 & 27.40 & 1.58 & 1.343 & 1527 & & \\
\hline \multirow[t]{7}{*}{976} & & 3 & 1.98 & 28.16 & 1.57 & 1.332 & 1513 & & \\
\hline & & 4 & 2.03 & 29.90 & 1.55 & 1.353 & 1560 & & \\
\hline & & 5 & 2.03 & 28.38 & 1.55 & 1.330 & $15 \overline{92}$ & & \\
\hline & \multirow{3}{*}{\multicolumn{2}{|c|}{$\begin{array}{r}\text { Average: } \\
\text { Standard Deviation: } \\
\text { Coefficient of Variance: }\end{array}$}} & 2.02 & 28.46 & 1.56 & 1.339 & 1548 & & \\
\hline & & & 0.03 & 1.05 & 0.02 & 0.011 & 36 & & \\
\hline & & & $1.7 \%$ & $3.7 \%$ & $1.0 \%$ & $0.8 \%$ & $2.3 \%$ & & \\
\hline & \multirow{4}{*}{ RDC-1586-D2 } & 1 & 1.70 & 32.38 & 1.00 & 0.348 & 1608 & & \\
\hline \multirow{7}{*}{977} & & 2 & 1.69 & 33.09 & 1.03 & 0.337 & 1636 & & \\
\hline & & 3 & 1.60 & 31.15 & 1.08 & 0.521 & 1633 & & \\
\hline & & 4 & 1.71 & 30.76 & 1.07 & 0.490 & 1619 & & \\
\hline & \multirow{3}{*}{\multicolumn{2}{|c|}{$\begin{array}{r}\text { Average: } \\
\text { Standard Deviation: } \\
\text { Coefficlent of Variance: } \\
\end{array}$}} & 1.67 & 31.85 & 1.04 & 0.424 & 1624 & & \\
\hline & & & 0.05 & 1.08 & 0.04 & 0.095 & 13 & & \\
\hline & & & $3.0 \%$ & $3.4 \%$ & $3.4 \%$ & $22.4 \%$ & $0.8 \%$ & & \\
\hline & \multirow{4}{*}{ RDC-1592-D2 } & 1 & 1.91 & 33.64 & 1.26 & 0.756 & 1736 & & \\
\hline \multirow[t]{7}{*}{978} & & 2 & 2.01 & 34.32 & 1.27 & 0.732 & 1653 & & \\
\hline & & 3 & 1.97 & 35.86 & & 0.757 & 1667 & & \\
\hline & & 4 & 1.94 & 36.89 & 1.32 & 0.747 & 1695 & & \\
\hline & \multirow{3}{*}{\multicolumn{2}{|c|}{$\begin{array}{r}\text { Average: } \\
\text { Standard Deviation: } \\
\text { Coefficient of Variance: }\end{array}$}} & 1.96 & 35.18 & 1.28 & 0.748 & 1688 & & \\
\hline & & & 0.04 & 1.47 & 0.03 & 0.012 & 36 & & \\
\hline & & & $2.2 \%$ & $4.2 \%$ & $2.1 \%$ & $1.5 \%$ & $2.2 \%$ & & \\
\hline & \multirow{4}{*}{ RDC-1593-D2 } & 1 & 2.09 & 33.97 & 1.27 & 0.409 & 1719 & & \\
\hline \multirow[t]{6}{*}{979} & & 2 & 2.12 & 33.74 & 1.22 & 0.413 & 1766 & & \\
\hline & & 3 & 2.11 & 32.60 & $1.23^{\circ}$ & 0.394 & 1720 & & \\
\hline & & 4 & $220^{\circ}$ & 33.82 & 1.36 & 0.396 & 1748 & & \\
\hline & \multirow{3}{*}{\multicolumn{2}{|c|}{$\begin{array}{r}\text { Average: } \\
\text { Standard Deviation: } \\
\text { Coefficlent of Variance: }\end{array}$}} & 2.13 & 33.53 & 1.27 & 0.403 & $1738^{\circ}$ & & \\
\hline & & & 0.05 & 0.63 & 0.06 & 0.009 & 23 & & \\
\hline & & & $2.3 \%$ & $1.9 \%$ & $4.9 \%$ & $2.3 \%$ & $1.3 \%$ & & \\
\hline
\end{tabular}

- Emissions Unit: grams/mile 


\section{Emissions Data Summary}

\begin{tabular}{|c|c|c|c|c|c|c|c|c|c|}
\hline Test ID & WvU Ref Num & Run ID & $\mathrm{CO}$ & NOX & HC & $\overline{P M}$ & $\mathrm{CO} 2$ & CH4 & NMHC \\
\hline \multirow{6}{*}{973} & \multirow{5}{*}{ RDC-1501-LNG } & 1 & 7.82 & & 22.89 & 0.069 & 1722 & 20.27 & 0.68 \\
\hline & & 2 & $7.83^{-}$ & 2.67 & 19.65 & 0.059 & 1707 & 17.34 & 0.64 \\
\hline & & 3 & 7.53 & 2.80 & 20.06 & 0.058 & 1719 & 17.71 & 0.64 \\
\hline & & 4 & $\overline{7.41}$ & 3.39 & 20.16 & 0.062 & 1661 & 17.83 & 0.62 \\
\hline & & 5 & 7.43 & 3.12 & 19.08 & 0.075 & $1659^{\circ}$ & 16.82 & 0.64 \\
\hline & \multirow{2}{*}{$\cdots$} & 6 & & 3.34 & 21.45 & 0.082 & $1656^{\circ}$ & 18.97 & $0.66^{\circ}$ \\
\hline ......... & & Average: & 7.60 & 3.08 & 20.55 & 0.064 & 1687 & 18.16 & 0.65 \\
\hline \multirow[t]{2}{*}{$\vdots$} & \multirow{2}{*}{\multicolumn{2}{|c|}{$\begin{array}{r}\text { Standard Deviation: } \\
\text { Coefficlent of Variance: }\end{array}$}} & 0.21 & 0.32 & 1.39 & 0.008 & 32 & 1.25 & 0.02 \\
\hline & & & $2.7 \%$ & $10.4 \%$ & $6.8 \%$ & $10.1 \%$ & $1.8 \%$ & $6.8 \%$ & $3.2 \%$ \\
\hline \multirow{4}{*}{974} & \multirow{4}{*}{ RDC-1502-LNG } & 1 & 6.70 & 2.93 & 17.40 & 0.042 & 1601 & 15.37 & 0.56 \\
\hline & & 2 & 6.57 & 2.89 & 15.99 & 0.039 & 1614 & 14.13 & 0.51 \\
\hline & & 3 & 6.75 & 3.01 & 16.20 & 0.042 & 1559 & 14.32 & 0.51 \\
\hline & & 4 & 6.52 & 2.68 & 16.49 & 0.039 & 1577 & 14.59 & $0.50^{\circ}$ \\
\hline & \multirow{3}{*}{\multicolumn{2}{|c|}{$\begin{array}{r}\text { Average: } \\
\text { Standard Deviation: } \\
\text { Coefficient of Variance: }\end{array}$}} & 6.64 & 2.88 & 16.52 & $0.041^{1}$ & 1588 & 14.60 & 0.62 \\
\hline & & & 0.11 & 0.14 & 0.62 & 0.002 & 25 & 0.54 & 0.03 \\
\hline & & & $1.6 \%$ & $5.0 \%$ & $3.8 \%$ & $4.3 \%$ & $1.5 \%$ & $3.7 \%$ & $5.3 \%$ \\
\hline \multirow{9}{*}{975} & \multirow{6}{*}{ RDC-1503-LNG } & 1 & 7.03 & 4.21 & 17.60 & 0.064 & 1563 & 15.49 & 0.61 \\
\hline & & 2 & 8.10 & 4.96 & 18.12 & 0.052 & 1497 & 16.04 & 0.55 \\
\hline & & 3 & 6.60 & 5.33 & 15.96 & 0.055 & 1543 & 14.04 & 0.57 \\
\hline & & 4 & 8.61 & 5.64 & 17.22 & 0.060 & 1558 & 15.18 & 0.58 \\
\hline & & 5 & 8.45 & 8.67 & 17.01 & 0.054 & 1598 & 15.02 & 0.54 \\
\hline & & 6 & 8.16 & 6.62 & 17.38 & 0.055 & 1623 & 15.31 & 0.59 \\
\hline & \multirow{3}{*}{\multicolumn{2}{|c|}{$\begin{array}{r}\text { Average: } \\
\text { Standard Deviation: } \\
\text { Coefficient of Variance: }\end{array}$}} & 7.83 & 5.57 & 17.22 & 0.056 & 1564 & 15.18 & 0.57 \\
\hline & & & 0.82 & 0.86 & 0.72 & 0.004 & 44 & 0.66 & 0.03 \\
\hline & & & $10.4 \%$ & $17.2 \%$ & $4.2 \%$ & $7.6 \%$ & $2.8 \%$ & $4.4 \%$ & $4.7 \%$ \\
\hline \multirow{9}{*}{968} & \multirow{6}{*}{ RDC-1504-LNG } & 1 & 7.17 & 13.21 & 15.32 & 0.069 & 1647 & 13.49 & 0.52 \\
\hline & & 2 & 8.42 & 12.86 & 15.55 & 0.070 & 1631 & \\
\hline & & 3 & 8.04 & 11.20 & 15.45 & 0.069 & 1620 & 13.59 & 0.54 \\
\hline & & 4 & 7.36 & 1241 & 14.54 & 0.053 & 1562 & 12.68 & 0.62 \\
\hline & & 5 & 7.62 & 16.57 & $14.79^{-}$ & 0.057 & 1682 & & \\
\hline & & 6 & 7.67 & $11.63^{\circ}$ & $14.20^{\circ}$ & 0.053 & 1645 & $1 \overline{2} .41$ & 0.59 \\
\hline & & Average: & 7.71 & 12.81 & 14.97 & 0.064 & 1631 & 13.05 & 0.57 \\
\hline & Standard & Deviation: & 0.46 & 1.55 & 0.55 & 0.007 & 40 & 0.59 & 0.04 \\
\hline & Coefficient o & Variance: & $5.9 \%$ & $12.1 \%$ & $3.6 \%$ & $11.3 \%$ & $2.4 \%$ & $4.5 \%$ & $7.5 \%$ \\
\hline \multirow{9}{*}{969} & \multirow{6}{*}{ RDC-1505-LNG } & 1 & 7.53 & 4.33 & 20.62 & 0.101 & 1706 & 18.21 & 0.66 \\
\hline & & 2 & $7 . \overline{36}$ & 4.60 & 19.04 & 0.122 & 1692 & 16.74 & 0.69 \\
\hline & & 3 & 7.22 & 5.13 & 18.75 & 0.096 & 1702 & 16.50 & 0.65 \\
\hline & & 4 & 7.00 & 5.23 & 18.18 & 0.085 & 1716 & 15.95 & 0.69 \\
\hline & & 5 & 7.04 & 5.16 & 17.09 & 0.088 & 1722 & 14.99 & 0.65 \\
\hline & & 6 & 6.77 & 5.57 & 16.75 & 0.077 & 1644 & 14.69 & 0.64 \\
\hline & & Average: & 7.16 & 5.00 & 18.41 & 0.095 & 1697 & 18.18 & 0.66 \\
\hline & Standard & Deviatlon: & 0.27 & 0.45 & 1.41 & 0.016 & 28 & 1.28 & 0.02 \\
\hline & Coefficient 0 & Variance: & $3.8 \%$ & $9.0 \%$ & $7.7 \%$ & $16.5 \%$ & $1.7 \%$ & $7.9 \%$ & $3.2 \%$ \\
\hline \multirow{4}{*}{970} & & 1 & 6.93 & 4.45 & 15.39 & 0.048 & 1669 & 13.53 & 0.56 \\
\hline & & 2 & 6.88 & 4.56 & 16.52 & 0.048 & 1654 & 14.54 & 0.58 \\
\hline & 1506-LNG & 3 & 6.77 & 4.34 & $15.20^{\circ}$ & $0.060^{\circ}$ & 1596 & 13.35 & 0.55 \\
\hline & & 4 & $6.70^{\circ}$ & 4.66 & 14.83 & 0.052 & 1589 & 13.00 & 0.57 \\
\hline & & Average: & 6.82 & 4.50 & 15.48 & 0.052 & 1627 & 13.60 & 0.56 \\
\hline & Stan & Deviation: & 0.11 & 0.14 & 0.73 & 0.006 & 40 & 0.68 & 0.01 \\
\hline & Coetriclent & Variance: & $1.6 \%$ & $3.1 \%$ & $4.7 \%$ & $10.8 \%$ & $2.5 \%$ & $4.9 \%$ & $2.0 \%$ \\
\hline
\end{tabular}


Emission Summary for LNG Powered Tractors in Sacramento, CA

\begin{tabular}{|c|c|c|c|c|c|c|c|c|c|c|c|c|c|}
\hline Test ID & $\therefore$ WuU Ref Num & Cycle & Test Date & $\therefore$ Fuel & $\mathrm{CO}$ & $\mathrm{NO}_{x}$ & $\mathrm{HC}$ & $P M$ & $\mathrm{CO}_{2}$ & MPG & BTU/mile & $\mathrm{CH}_{4}$ & NMHC \\
\hline 973 & RDC-1501-LNG-5MILES-R & 5 Mile Route & $7 / 31 / 97$ & LNG & 7.60 & 3.06 & 20.55 & 0.06 & 1687 & 4.28 & 30024 & 18.16 & 0.65 \\
\hline 974 & ROC-1502-LNG-5MILES-R & 5 Mile Route & $8 / 1 / 97$ & LNG & 6.64 & 2.88 & 16.52 & 0.04 & 1588 & 4.57 & 28103 & 14.60 & 0.52 \\
\hline 975 & RDC-1503-LNG-5MILES-R & 5 Mile Route & $8 / 2 / 97$ & LNG & 7.83 & 5.57 & 17.22 & 0.06 & 1564 & 4.63 & 27755 & $15 . \overline{18}$ & 0.57 \\
\hline 968 & RDC-1504-LNG-5MILES & 5 Mille Route & $7 / 25 / 97$ & LNG & 7.71 & 12.81 & 14.97 & 0.06 & 1631 & 4.46 & 28799 & 13.05 & 0.57 \\
\hline 969 & RDC-1505-LNG-5MILES & 5 Mile Route & $7 / 26 / 97$ & LNG & 7.16 & 5.00 & 18.41 & 0.09 & 1697 & 4.27 & 30069 & 16.18 & 0.66 \\
\hline 970 & RDC-1506-LNG-5MilLES & 5 Mile Route & $7 / 28 / 97$ & LNG & 6.82 & 4.50 & 15.48 & 0.05 & 1627 & 4.48 & 28729 & 13.60 & 0.56 \\
\hline 971 & RDC-1507-LNG-5MILES & 5 Mile Route & 7/29/97 & LNG & 6.58 & 3.65 & 18.31 & 0.07 & 1700 & 4.27 & 30100 & 16.13 & 0.62 \\
\hline 972 & RDC-1508-LNG-5MILES & 5 Mile Route & $7 / 30 / 97$ & LNG & 6.60 & 3.79 & 18.64 & 0.09 & 1765 & 4.12 & $\overline{31222}$ & 16.42 & 0.64 \\
\hline & & & & Average $\rightarrow$ & 7.12 & 5.16 & 17.51 & 0.07 & 1657 & 4.39 & 29350 & 15.41 & 0.60 \\
\hline
\end{tabular}

Emission Summary for diesel Powered Tractors in Sacramento, CA

\begin{tabular}{|c|c|c|c|c|c|c|c|c|c|c|c|}
\hline Test í. & WMU Ref Num & Cycle & Test Date & Fuel & $\mathrm{co}$ & $\mathrm{NO}_{\mathrm{x}}$ & $\mathrm{HC}$ & $P M$ & $\mathrm{CO}_{2}$ & MPG & BTU/mile \\
\hline 979 & RDC-1579-D2-5MILES-R & 5 Mile Route & $8 / 8 / 97$ & $\overline{D 2}$ & 2.13 & 33.53 & 1.27 & 0.40 & 1738 & 5.83 & 22301 \\
\hline 976 & RDC-1586-D2-5MILES-R2 & 5 Mile Route & $8 / 4 / 97$ & D2 & 2.02 & 28.46 & 1.56 & 1.34 & 1548 & 6.54 & $19 \overline{880}$ \\
\hline 978 & RDC-1592-D2-5MILES-R & 5 Mile Route & $8 / 7197$ & D2 & 1.96 & 35.18 & 1.28 & 0.75 & 1688 & 6.01 & 21652 \\
\hline 977 & RDC-1593-D2-5MILES-R & 5 Mile Route & $8 / 6 / 97$ & D2 & 1.67 & 31.85 & 1.04 & 0.42 & 1624 & 6.24 & 20822 \\
\hline & & & & Averages $>$ & 1.85 & 32.25 & 1.29 & 0.73 & 1648 & 6.16 & 21164 \\
\hline
\end{tabular}




\title{
WEST VIRGINIA UNIVERSITY Transportable Heavy Duty Vehicle Emissions Testing Laboratory
}

\author{
Exhaust Emissions Test Results Report of
}

Raley's Distribution Center (RDC) Tractors

\section{$\underline{\text { Submitted to }}$}

National Renewable Energy Laboratory (NREL) Raley's Distribution Center (RDC)

Department of Mechanical \& Aerospace Engineering

West Virginia University

Morgantown, WV 26506-6101

Phone: (304) 293-3111 


\section{Contents}

\section{Introduction}

2 Test Circumstance and Characteristics

2.1 Transportable Laboratory

2.2 Test Vehicles

2.3 Test Procedure

3 Test Results

3.1 Emissions Summary and Comparison

3.2 Emissions Comparison (Table 1)

3.3 Short Report 


\section{Introduction}

West Virginia University (WVU) Transportable Heavy Duty Emissions Testing Laboratory, through the funding from the Department of Energy, gathers emissions data from transit fleets operating on alternative and conventional fuels. In cooperation with United States Department of Energy, Office of Transportation Technologies (OTT), The University has designed, constructed and now operates two Transportable Heavy Duty Vehicle Emissions Testing Laboratories which travel to transit agency and trucking facilities across North America to measure vehicle emissions on sites. These emissions data are placed in a database maintained by the National Renewable Energy Laboratories' (NREL) transit bus and heavy-duty truck evaluation programs. Detailed information pertaining to the Transportable Laboratory, NREL database and OTT can be found at through the Internet at the following web sites:

NREL database: http://www.afdc.nrel.gov/web view/emishdv.html (Heavy-duty truck program) http://www.afdc.nrel.gov/web view/emisbus.html (Transit bus program)

WVU Transportable Laboratory: $\quad$ http://www.cemr.wvu.edw/ wwwatf/TransportableLaboratory.html OTT: $\quad$ http://www.ott.doe.gov/

\section{Test Conditions and Procedures}

\subsection{Transportable Laboratory}

The Transportable Laboratories were constructed to gather emissions data from in-use heavyduty vehicles. The laboratories are fully self-contained units that can be transported to and set up at or near transit agency maintenance facilities. Emissions tests are performed on location and vehicles are returned to the transit agency, usually within one day. Several technical papers (SAE 961082, SAE 951016, and SAE 952746) have been presented the design of the two laboratories and on emissions data from both conventional and alternatively fueled vehicles.

The laboratory facility arrives on the test site pulled on two trailers, one being a box trailer containing equipment for emissions measurement, data acquisition and control, and the other, a flat bed semi-trailer carrying the power absorber unit. The flat bed is lowered to the ground to provide a chassis dynamometer platform. 
The vehicle to be tested is mounted onto the chassis dynamometer platform with the rear drive wheels positioned on free-rolling rollers. The outer wheels of the dual wheel set on each side of the vehicle are connected to the drive shafts of the dynamometer units located on each side of the vehicle. Each dynamometer unit consists of speed-increasing gearboxes with a power absorber and a flywheel set. In the case of the tandem axle tractors discussed in this report, the power divider was locked and power was taken from the forward rear axle. The flywheel sets consist of a series of selectable discs to allow simulation of vehicle inertia. During the test cycle, torque cells and speed transducers in the power absorber drive train measure the actual vehicle load (torque) and speed. The trucks tested in this program were exercised through the Five-Mile Route. This cycle was a modification of WVU Five-Peak truck cycle that was originally described in SAE papers 941946 and 951016 . The Five-Mile Route is similar to the original WVU Five-Peak truck cycle that has five steady speeds, while it differs from Five-Peak cycle when it accelerates to the steady speed using the highest possible acceleration for each peak speed. To make test results comparable to each other, the driving distance for both of these cycles is held at five miles and the driving duration including idle time is held at 900.1 seconds. A sample actual driving speed for LNG tractor 1501 is shown in figure 1.

The full exhaust from the tail pipe of the test vehicle is ducted to a $45 \mathrm{~cm}$ diameter dilution tunnel on top of the emissions trailer. The exhaust is mixed with dilution air and the flow is controlled using a blower with critical flow venturi. Heated sampling probes send diluted exhaust to a number of different gas analysis instruments, via heated lines. Levels of carbon dioxide $\left(\mathrm{CO}_{2}\right)$, carbon monoxide $(\mathrm{CO})$, oxides of nitrogen $\left(\mathrm{NO}_{\mathrm{x}}\right)$ and hydrocarbons $(\mathrm{HC})$ are measured continuously. A gravimetric measurement of particulate matter $(\mathrm{PM})$ is obtained using $70 \mathrm{~mm}$ filters, weighed after conditioning for temperature and humidity in an environmental chamber.

In this study, bags of the natural gas fueled vehicles diluted exhaust were shipped to West Virginia University for analysis using a Varian 3600 gas chromatograph. The column (J\&W GSAlumna) used for measurement had an inner diameter of $0.53 \mathrm{~mm}$ and a length of 50 meters. The volume injected was $2 \mathrm{ml}$ and the oven temperature was for $40^{\circ} \mathrm{C}$ held for 2.5 minutes, then ramped to $200^{\circ} \mathrm{C}$ at $20^{\circ} \mathrm{C}$ per minute then held for 15 minutes. A flame ionization detector (FID) was used and the temperature set at $250^{\circ} \mathrm{C}$. Methane and propane standards were used to measure the response factors. Methane response was used to quantify methane and propane response was used to quantify all other non-methane hydrocarbons. These data are used to distinguish methane from non-methane hydrocarbons in the exhaust and ultimately yield information on the atmospheric reactivity of the exhaust hydrocarbons. 


\subsection{Test Vehicles}

\section{LNG Fueled Test Vehicles:}

A total of eight tractor trucks (1997 Kenworth) were tested in Sacramento, CA, each with the Cummins 6 cylinder 10-liter engine. The engines (CPL 2110) were spark ignited, turbo-charged (air to air), with a compression ratio of 16.1:1.

\section{Diesel Fueled Test Vehicles:}

A total of three tractor trucks (1995 Kenworth) were tested in Sacramento, CA, each with the Cummins M-11E+ diesel engine (CPL 2140) derived from the L-10 block, with displacement increased to 10.8 liters. The diesel vehicles had older vehicle and engine model years and higher mileage accumulation than the LNG vehicles. The diesel vehicles had splitter transmissions while the LNG vehicles had ranger transmissions.

\subsection{Test Procedure}

Test procedure included calibration of equipment, vehicle inspection, actual vehicle testing, data reduction, and data analysis.

In order to make sure that the analyzers and the associated systems were functioning properly and providing measurements accurately, all instruments were calibrated or adjusted daily. Propane injections confirmed reliability of the dilution tunnel system.

Prior to the actual testing, all gearboxes in the power train of the dynamometer were warmed up to minimize loading variability due to the viscosity of the oil. Each test includes several repeat test-runs in order to guarantee that the exhaust emissions measured are a true representation of the test vehicle.

The emissions data reported for each vehicle test are the average values of at least three repeat test-runs. All test runs were executed with the vehicle at operating temperature (no cold-start tests were performed).

The following procedures were adopted for the actual vehicle testing:

1) The vehicle was inspected prior to testing; 
2) The vehicle was positioned and mounted on the chassis dynamometer

3) The vehicle was driven while on the test bed to warm-up the gearboxes of the power train of the dynamometer until they reached $100^{\circ} \mathrm{F}$, also ensuring that the vehicle's engine is at operational temperature before testing.

4) A practice test cycle was performed to allow the driver to become familiar with vehicle characteristics and to insure proper range settings of analyzers;

5) After completion of the practice run or one test run, the vehicle transmission was set to neutral and the engine was left in this condition for 17 minutes into a 20 minute soak period;

6) At 17 minutes into the soak period, the vehicle was driven though three of the CBD ramps to expel collected constituents in the exhaust system. Twenty seconds after completion of the final practice ramp, data collection was initiated.

7) 5) and 6) were repeated at least two times.

All emissions data, except for PM and non-methane hydrocarbons, were obtained by continuous readings and the results were integrated over the complete test cycle. PM emissions were measured gravimetrically using a secondary dilution filter sampling of the primary diluted exhaust. Non-methane hydrocarbons were measured by a gas chromatograph laboratory for all the CNG vehicles and LNG vehicles.

During a test, the driver was provided with a visual trace of the scheduled test route on an interface monitor. The speed trace was a representation of the test cycle being used. The driver followed the speed trace closely to minimize the errors introduced by irregular driving.

All of the vehicles were jacked up with hydraulic jacks placed on scales until the rear tires were no longer on the rollers of the test bed. The vehicle was then lowered onto the rollers until the vehicle was centered on the test bed.

\subsection{Emissions Summary and Comparison}

This section discusses the contrast between eight tractor trucks with new Cummins L-10 natural gas engines and three M-11 diesel control tractor trucks in Sacramento, CA. The LNG vehicles had an average mileage of 43,000 miles, while the diesel control vehicles' average was 196,000 miles. Table 1 presents the data acquired. PM from the LNG trucks was low, and it was about the one-third of the 
diesel PM level. The level of CO for the LNG trucks was higher than that for the diesel trucks, with the average about 3.5 times of the average for the diesel trucks.

The average diesel truck $\mathrm{NO}_{\mathrm{x}}$ emissions were $19.6 \mathrm{~g} / \mathrm{mile}$. Modern electronic diesel engine control strategy provides for advanced injection timing under warm up conditions, and the advanced timing causes higher $\mathrm{NO}_{\mathrm{x}}$. The average $\mathrm{NO}_{\mathrm{x}}$ emissions for the eight $\mathrm{LNG}$ trucks was $5.25 \mathrm{~g} / \mathrm{mile}$, well below the value for the diesel trucks. These NOx levels are so low as to be noteworthy in the database; they were also low in the previous year's test.

Non-methane hydrocarbon emissions represented $3.1 \%$ of the total hydrocarbon mass emitted for the LNG trucks. Average NMHC, at $0.62 \mathrm{~g} /$ mile, was lower than the diesel truck $\mathrm{HC}$ emissions, at 1.70 g/mile.

Energy-equivalent fuel consumption of the CNG trucks was $36.5 \%$ poorer than for the diesel trucks this year. It is also noticed that the fuel consumption of the diesel trucks stayed the same as tested in the previous year, while for the LNG trucks, it was 10\% more this year. This could be due to the weather, since tests were done in summer last year when ambient temperature was higher than this year. Trucks 1503, 1507 and 1508 have fuel economy close to that of last year.

A tune up service over a LNG truck 1501 dropped the emissions dramatically. This truck was - tested with the vehicle in original condition as received from the fleet owner in test sequence number 1047. It was re-tested in test sequence number 1058 after one of the spark plugs was found to be in poor condition and replaced with a new one. $\mathrm{CO}, \mathrm{HC}$ and $\mathrm{PM}$ emissions decreased after this tune up service.

In summary, the LNG powered trucks showed their ability to return low $\mathrm{NO}_{\mathrm{x}}$ and $\mathrm{PM}$ emissions in comparison to diesel counterparts.

\subsection{Short Report}

The short report shows the vehicle information, vehicle engine information, emissions data in grams/mile, and fuel economy for each test run, average emissions over all test runs, and brief comments for each test in a compact format on one page. The odometer mileage reading or hub mileage reading in the short reports is rounded to the nearest 100 miles.

Symbols used in Short Report Emissions data result table: 
a A value was measured and identified as an apparent outliar, and therefore is not reported and not used to compute other parameters or the average values.

b The residual hydrocarbon emissions (RHC) is calculated from the difference between the methanol $\left(\mathrm{CH}_{3} \mathrm{OH}\right)$ and the FID-HC concentrations. For $100 \%$ alcohol fuels, the value of RHC is small and due to experimental variations, it may be measured as positive or negative but can best be assumed to be zero.

c A value cannot be calculated because the parameters required for calculation are not available.

d A value of coefficient variance (CV\%) is not meaningful because the average value is too small or not available. A significant coefficient of variance may exist for PM from CNG vehicles, where the PM is at very low levels. Note that CNG PM is more than an order of magnitude less than PM usually measured from diesel vehicles. Similarly some modern diesel vehicles will yield very low hydrocarbon emissions.

Component codes used in the short report data table:

CO: $\quad$ Carbon monoxide in grams/mile

$\mathrm{CO}_{2}$ : $\quad$ Carbon dioxide in grams/mile

$\mathrm{NO}_{\mathbf{x}}$ : $\quad$ Oxides of nitrogen in grams/mile

FIDHC: Total hydrocarbon measured by HFID in grams/mile. For CNG and LNG vehicle test, unburned methane is included and no HFID response factor was corrected.

PM: $\quad$ Particulate matter in grams/mile

$\mathrm{CH}_{4}$ : Unburned methane emissions in grams/mile

NMHC: Non-methane hydrocarbon in grams/mile

mile/gal: Calculated fuel economy in mile/gallon. For NG fueled vehicles, MPG means miles per equivalent gallon diesel. In this table, 137 cubic feet $\mathrm{CNG}$ at standard temperature and pressure (STP) is equivalent to 1 gallon of \#1 diesel.

BTU/mile: Calculated fuel energy used by the vehicle, in BTU/mile.

Miles: Total actual driving distance for a test run

All short reports for RDC trucks are included in this report. 
Test Sequence Number: 1046

WVU Test Reference Number: WVU-TEDDY2-D2-5MILES

Fleet Owner Full Name

Fleet Address

Fleet Address (City, State, Zip)

Vehicle Type

Vehicle ID Number (VIN)

Vehicle Manufacturer

Vehicle Model Year

Gross Vehicle Weight (GWW) (lb.)

Vehicle Total Curb Weight (lb.)

Vehicle Tested Weight (lb.)

Odometer Reading (mile)

Transmission Type

Transmission Configuration

Number of Axles

Engine Type
Engine ID Number
Engine Displacement (Liter)
Number of Cylinders
Engine Rated Power (hp)

Primary Fuel

Test Cycle

Test Date

Engineer

Driver
West Virginia University

MAE Dept., P. O. Box 6106

Morgantown, WW 26506

Tractor Truck

1FUPYSYB7FH258124

Freightliner

1985

80000

12000

42000

748400

Manual

9-Speed

3

Caterpillar 3406B

7FB30060

14

6

350

D2

5 Mile Route

2/18/98

W. Xie

B. Davis

Emissions Results (g/mile)

Fuel Economy

\begin{tabular}{|c|c|c|c|c|c|c|c|c|}
\hline Rún SẼ Nố & 60 & $\mathrm{NO}_{x \alpha}$ & \&FIDHC & 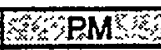 & $\mathrm{CO}$ & Cunitélgäl's & BTU/mile & Walles \\
\hline $1046-3$ & 15.4 & 30.1 & 3.53 & 3.17 & 2166 & 4.62 & 28128 & 5.00 \\
\hline $1046-4$ & 17.1 & 29.3 & 5.04 & 3.98 & 2118 & 4.71 & 27605 & 5.00 \\
\hline $1046-5$ & 13.8 & 30.1 & 2.68 & 2.34 & 2120 & 4.73 & 27470 & 5.01 \\
\hline $1046-6$ & 16.2 & 29.9 & 4.71 & 3.64 & 2133 & 4.68 & 27767 & 5.00 \\
\hline 1046 Average & 15.6 & 29.8 & 3.99 & 3.28 & 2134 & 4.69 & 27742 & 5.00 \\
\hline Std. Dev. & 1.4 & 0.4 & 1.09 & 0.71 & 22 & 0.05 & 284 & 0.00 \\
\hline CV\% & 9.1 & 1.3 & 27.3 & 21.6 & 1.0 & 1.0 & 1.0 & 0.0 \\
\hline
\end{tabular}

Test Purpose:

Measurement of exhaust constituents from WVU Transportable Heavy Duty Emissions Laboratory-2 control vehicle for comparison of emissions on the same vehicle at different locations.

\section{Special Procedures:}

The vehicle was serviced 3000 miles prior to this test.

\section{Observations:}

$\overline{\mathrm{HC}}$ and $\mathrm{PM}$ emissions were very high compared to emissions measured previously from this vehicle at other test sites. 
Test Sequence Number: 1047

WVU Test Reference Number: RDC-1501-LNG-5MILES

Fleet Owner Full Name

Fleet Address

Fleet Address (City, State, Zip)

Vehicle Type

Vehicle ID Number (VIN)

Vehicle Manufacturer

Vehicle Model Year

Gross Vehicle Weight (GWW) (lb.)

Vehicle Total Curb Weight (lb.)

Vehicle Tested Weight (lb.)

Odometer Reading (mile)

Transmission Type

Transmission Configuration

Number of Axles

Engine Type

Engine ID Number

Engine Displacement (Liter)

Number of Cylinders

Engine Rated Power (hp)

Primary Fuel

Test Cycle

Test Date

Engineer

Driver
Raley's Distribution Center

4061 Gateway Park Blvd.

Sacramento, CA 95834

Tractor Truck

1XKDD59XOVR745373

Kenworth

1997

80000

16520

42000

37200

Manual

10-Speed

3

Cummins GL-10-300E

34845353

10

6

300

LNG

5 Mile Route

$2 / 19 / 98$

W. Xie

B. Davis
Emissions Results (g/mile)

Fuel Economy

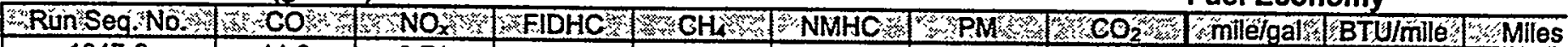

\begin{tabular}{|c|c|c|c|c|c|c|c|c|c|c|c}
\hline $1047-2$ & 11.0 & 3.71 & 83.5 & 75.7 & 0.70 & 0.13 & 1916 & 3.58 & 35850 & 5.01 \\
\hline $1047-3$ & 10.9 & 3.68 & 92.3 & 83.8 & 0.66 & 0.11 & 1970 & 3.48 & 36925 & 5.02 \\
\hline $1047-4$ & 9.8 & 3.83 & 76.0 & 69.0 & 0.64 & 0.09 & 1925 & 3.60 & 35730 & 5.00 \\
\hline $1047-5$ & 14.7 & 5.66 & 167.4 & 152.5 & 0.76 & 0.12 & 2022 & 3.25 & 39492 & 5.01 \\
\hline $1047-6$ & 13.9 & 3.59 & 129.5 & 117.8 & 0.79 & 0.17 & 1995 & 3.35 & 38355 & 5.00 \\
\hline & & & & & & & & & & \\
\hline 1047 Average & 12.0 & 4.09 & 109.8 & 99.7 & 0.71 & 0.13 & 1966 & 3.45 & 37270 & 5.01 \\
\hline Std. Dev. & 2.1 & 0.88 & 38.2 & 35.0 & 0.07 & 0.03 & 45 & 0.15 & 1629 & 0.01 \\
\hline CV\% & 17.5 & 21.5 & 34.8 & 35.0 & 9.3 & 22.9 & 2.3 & 4.3 & 4.4 & 0.1 \\
\hline
\end{tabular}

\section{Test Purpose:}

Collection of emissions data from LNG fueled vehicles for use in comparison with similar, diesel powered vehicles.

\section{Special Procedures:}

Dilute bag was replaced with a new one the day before the test. It rained heavily during the test. This vehicle was re-tested in test number 1058. See comments in test sequence number 1058.

\section{Observations:}

Driver complained not being able to hold the speed during the test. Low fuel pressure light was on during the 5 th test, even though the fuel tank was above half. $\mathrm{HC}$ emissions were very high and out of the analyzer range, $\mathrm{HC}$ dilute bag results were reported. 
Test Sequence Number: 1048

WVU Test Reference Number: RDC-1502-LNG-5MILES

Fleet Owner Full Name

Fleet Address

Fleet Address (City, State, Zip)

Vehicle Type

Vehicle ID Number (VIN)

Vehicle Manufacturer

Vehicle Model Year

Gross Vehicle Weight (GWW) (lb.)

Vehicle Total Curb Weight (lb.)

Vehicle Tested Weight (lb.)

Odometer Reading (mile)

Transmission Type

Transmission Configuration

Number of Axles

Engine Type

Engine ID Number

Engine Displacement (Liter)

Number of Cylinders

Engine Rated Power (hp)

Primary Fuel

Test Cycle

Test Date

Engineer

Driver
Raley's Distribution Center

4061 Gateway Park Blvd.

Sacramento, CA 95834

Tractor Truck

1XKDD59X2VR745374

Kenworth

1997

80000

16380

42000

38600

Manual

10-Speed

3

Cummins GL-10-300E

34847019

10

6

300

LNG

5 Mile Route

2/20/98

W. Xie

B. Davis

Emissions Results (g/mile)

Fuel Economy

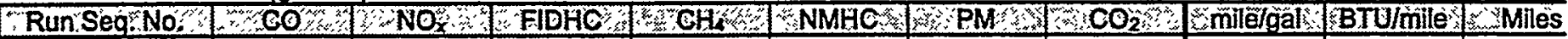

\begin{tabular}{|c|c|c|c|c|c|c|c|c|c|c}
\hline $1048-2$ & 8.98 & 4.11 & 26.6 & 23.8 & 0.60 & 0.046 & 1793 & 4.00 & 32142 & 5.02 \\
\hline $1048-4$ & 8.72 & 4.56 & 28.6 & 25.6 & 0.61 & 0.047 & 1832 & 3.91 & 32891 & 5.01 \\
\hline $1048-5$ & 8.32 & 4.41 & 25.3 & 22.6 & 0.62 & 0.038 & 1827 & 3.94 & 32641 & 5.00 \\
\hline $1048-6$ & 7.72 & 5.49 & 21.3 & 18.8 & 0.71 & 0.031 & 1813 & 3.99 & 32201 & 5.00 \\
\hline & & & & & & & & & & \\
\hline 1048 Average & 8.44 & 4.65 & 25.5 & 22.7 & 0.63 & 0.041 & 1816 & 3.96 & 32469 & 5.01 \\
\hline Std. Dev. & 0.55 & 0.60 & 3.1 & 2.9 & 0.05 & 0.008 & 17 & 0.04 & 359 & 0.01 \\
\hline CV\% & 6.5 & 12.8 & 12.1 & 12.6 & 8.3 & 18.8 & 1.0 & 1.1 & 1.1 & 0.2 \\
\hline
\end{tabular}

\section{Test Purpose:}

Collection of emissions data from LNG fueled vehicles for use in comparison with similar, diesel powered vehicles.

\section{Speclal Procedures:}

Background bag was replaced with a new one the day before the test.

\section{Observations:}

Driver complained that the truck was sluggish and under-powered. 
Test Sequence Number: 1051

WVU Test Reference Number: RDC-1504-LNG-5MILES-R

Fleet Owner Full Name

Fleet Address

Fleet Address (City, State, Zip)

Vehicle Type

Vehicle ID Number (VIN)

Vehicle Manufacturer

Vehicle Model Year

Gross Vehicle Weight (GWW) (lb.)

Vehicle Total Curb Weight (lb.)

Vehicle Tested Weight (lb.)

Odometer Reading (mile)

Transmission Type

Transmission Configuration

Number of Axles

Engine Type

Engine ID Number

Engine Displacement (Liter)

Number of Cylinders

Engine Rated Power (hp)

Primary Fuel

Test Cycle

Test Date

Engineer

Driver

\author{
Raley's Distribution Center \\ 4061 Gateway Park Blvd. \\ Sacramento, CA 95834 \\ Tractor Truck \\ 1XKDD59X6VR745376 \\ Kenworth \\ 1997 \\ 80000 \\ 16540 \\ 42000 \\ 51400 \\ Manual \\ 10-Speed \\ 3 \\ Cummins GL-10-300E \\ 34847352 \\ 10 \\ 6 \\ 300 \\ LNG \\ 5 Mile Route \\ 2/23/98 \\ W. Xie \\ B. Davis
}

Emissions Results (g/mile)

Fuel Economy

\begin{tabular}{|c|c|c|c|c|c|c|c|c|c|c|}
\hline RunSeg NoY & 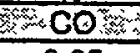 & KunO & FIDHC & FHa & X\&NMHC & SPM & $\mathrm{S}_{2} \mathrm{CO}_{3} \mathrm{~B}$ & mile/gal & BTU U/mile & Miles \\
\hline $1051-2$ & 8.05 & 4.67 & 22.1 & 19.5 & 0.72 & 0.049 & 1968 & 3.69 & 34848 & 5.00 \\
\hline $1051-3$ & 7.83 & 6.46 & 22.8 & 20.1 & 0.74 & 0.062 & 1908 & 3.80 & 33850 & 5.00 \\
\hline $1051-5$ & 9.45 & 6.95 & 50.5 & 45.4 & 0.85 & 0.061 & 1859 & 3.81 & 33769 & 4.98 \\
\hline $1051-6$ & 10.19 & 5.57 & 59.9 & 53.9 & 0.91 & 0.082 & 1879 & 3.73 & 34427 & 5.01 \\
\hline $1051-7$ & 8.98 & 5.59 & 44.7 & 40.1 & 0.80 & 0.062 & 1862 & 3.82 & 33679 & 5.01 \\
\hline & & & & & & & & & & \\
\hline 1051 Average & 8.90 & 5.85 & 40.0 & 35.8 & 0.80 & 0.063 & 1895 & 3.77 & 34115 & 5.00 \\
\hline Std. Dev. & 0.98 & 0.88 & 16.9 & 15.4 & 0.08 & 0.012 & 45 & 0.06 & 504 & 0.01 \\
\hline CV\% & 11.0 & 15.1 & 42.3 & 43.1 & 9.7 & 18.7 & 2.4 & 1.5 & 1.5 & 0.2 \\
\hline
\end{tabular}

\section{Test Purpose:}

Collection of emissions data from LNG fueled vehicles for use in comparison with similar, diesel powered vehicles.

\section{Special Procedures:}

Cummins representative plugged his device to log data during the test simultaneously. It was rainy during test. The vehicle was re-tested in test number 1056 under sunny weather to see the effect of rainy weather on emissions.

\section{Observations:}

Driver complained about the sluggishness of the vehicle. HC was out of analyzer range at 40MPH ramp for test run number 5,6 and $7, \mathrm{HC}$ dilute bag results were reported. 
Test Sequence Number: 1052

\section{WVU Test Reference Number: RDC-1503-LNG-5MILES}

Fleet Owner Full Name

Fleet Address

Fleet Address (City, State, Zip)

Vehicle Type

Vehicle ID Number (VIN)

Vehicle Manufacturer

Vehicle Model Year

Gross Vehicle Weight (GWW) (lb.)

Vehicle Total Curb Weight (lb.)

Vehicle Tested Weight (lb.)

Odometer Reading (mile)

Transmission Type

Transmission Configuration

Number of Axles

Engine Type

Engine ID Number

Engine Displacement (Liter)

Number of Cylinders

Engine Rated Power (hp)

Primary Fuel

Test Cycle

Test Date

Engineer

Driver
Raley's Distribution Center

4061 Gateway Park Blvd.

Sacramento, CA 95834

Tractor Truck

1XKDD59X4VR745375

Kenworth

1997

80000

16560

42000

46000

Manual

10-Speed

3

Cummins GL-10-300E

34847353

10

6

300

LNG

5 Mile Route

$2 / 24 / 98$

W. Xie

B. Davis

Emissions Results (g/mile)

Fuel Economy

\begin{tabular}{|c|c|c|c|c|c|c|c|c|c|c|}
\hline Run Sog No & $\cos$ & WNOKZ & FIDHC & $2 \mathrm{CH}_{4}$ & NAHC & 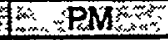 & $\mathrm{COO}_{2}$ & milêlgal & ETU/mile & Miles \\
\hline $1052-3$ & 7.80 & 7.04 & 15.7 & 13.7 & 0.68 & 0.048 & 1670 & 4.36 & 29495 & 5.00 \\
\hline $1052-5$ & 7.99 & 7.85 & 15.5 & c & c & 0.043 & 1644 & 4.42 & 29047 & 5.01 \\
\hline $1052-6$ & 8.17 & 8.62 & 16.1 & 14.2 & 0.55 & 0.046 & 1592 & 4.56 & 28198 & 5.00 \\
\hline $1052-7$ & 7.82 & 8.21 & 17.3 & 15.2 & 0.56 & 0.052 & 1626 & 4.46 & 28823 & 5.01 \\
\hline & & & & & & 0007 & 1023 & 145 & 28901 & \\
\hline 1052 Average & 7.95 & 7.93 & 16.1 & 14.4 & 0.60 & 0.041 & 7635 & $\frac{4.45}{0.09}$ & & 5.00 \\
\hline Std. Dev. & 0.17 & 0.67 & 0.8 & 0.8 & 0.07 & 0.004 & 33 & 0.08 & 540 & 0.01 \\
\hline CV\% & 2.2 & 8.5 & 4.9 & 5.5 & 12.0 & 7.9 & 2.0 & 1.9 & 1.9 & 0.1 \\
\hline
\end{tabular}

Test Purpose:

Collection of emissions data from LNG fueled vehicles for use in comparison with similar, diesel powered vehicles.

\section{Observations:}

Fuel tanking was leaking the day before the test when it was put on the test bed because it was too full. The leaking stopped before the test. 
Test Sequence Number: 1053

WVU Test Reference Number: RDC-1507-LNG-5MILES

Fleet Owner Full Name

Fleet Address

Fleet Address (City, State, Zip)

Vehicle Type

Vehicle ID Number (VIN)

Vehicle Manufacturer

Vehicle Model Year

Gross Vehicle Weight (GWW) (Ib.)

Vehicle Total Curb Weight (lb.)

Vehicle Tested Weight (lb.)

Odometer Reading (mile)

Transmission Type

Transmission Configuration

Number of Axles

Raley's Distribution Center

4061 Gateway Park Blvd.

Sacramento, CA 95834

Tractor Truck

1XKDD59X1VR745379

Kenworth

1997

80000

15585

42000

46000

Manual

10-Speed

3

Engine Type

Engine ID Number

Engine Displacement (Liter)

Number of Cylinders

Engine Rated Power (hp)

Cummins GL-10-300E

34849167

10

6

300

Primary Fuel

LNG

Test Cycle

5 Mile Route

Test Date

2/24/98

Engineer

W. Xie

Driver

B. Davis

Emissions Results (g/mile)

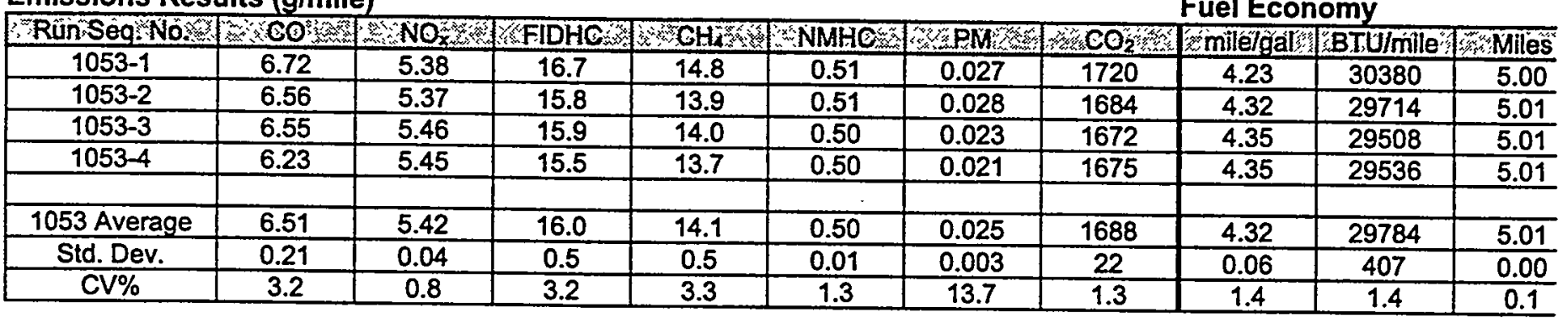

\section{Test Purpose:}

Collection of emissions data from LNG fueled vehicles for use in comparison with similar, diesel powered vehicles.

\section{Observations:}

The Lebow on the right side of the test bed (side 2), which had a short circuit inside and lost tracking since test number 1050 , started functioning during the third run of this test. It was re-calibrated before the fourth run. 
Test Sequence Number: 1054

WVU Test Reference Number: RDC-1506-LNG-5MILES

Fleet Owner Full Name

Fleet Address

Fleet Address (City, State, Zip)

Vehicle Type

Vehicle ID Number (VIN)

Vehicle Manufacturer

Vehicle Model Year

Gross Vehicle Weight (GWW) (Ib.)

Vehicle Total Curb Weight (Ib.)

Vehicle Tested Weight (lb.)

Odometer Reading (mile)

Transmission Type

Transmission Configuration

Number of Axles

Engine Type

Engine ID Number

Engine Displacement (Liter)

Number of Cylinders

Engine Rated Power (hp)

Primary Fuel

Test Cycle

Test Date

Engineer

Driver
Raley's Distribution Center

4061 Gateway Park Blvd.

Sacramento, CA 95834

Tractor Truck

1XKDD59XXVR745378

Kenworth

1997

80000

16520

42000

44700

Manual

10-Speed

3

Cummins GL-10-300E

34849166

10

6

300

LNG

5 Mile Route

2/25/98

W. Xie

B. Davis

Emissions Results (g/mile)

Fuel Economy

\begin{tabular}{|c|c|c|c|c|c|c|c|c|c|c|}
\hline Run Seg. No & $\mathrm{CO}$ & $\mathrm{NO}_{x}^{x}$ & FIDHC & CHars? & NMHCK & SPMES: & $\mathrm{CO}_{2}$ & miletgal & BTU/mile & Milès \\
\hline $1054-1$ & 7.19 & 5.03 & 17.9 & 15.8 & 0.54 & 0.035 & 1818 & 4.00 & 32110 & 5.00 \\
\hline $1054-2$ & 7.55 & 4.22 & 19.9 & 17.7 & 0.55 & 0.036 & 1882 & 3.86 & 33320 & 5.00 \\
\hline$\overline{1054-4}$ & 6.90 & 4.08 & 16.9 & 15.0 & 0.52 & 0.037 & 1843 & 3.95 & 32494 & $\overline{5.01}$ \\
\hline $1054-5$ & 6.59 & 4.90 & 16.0 & 14.1 & 0.54 & 0.042 & 1820 & 4.01 & 32048 & 5.00 \\
\hline $1054-6$ & 6.50 & 3.88 & 17.0 & 15.1 & 0.49 & 0.027 & 1843 & 3.96 & 32487 & 5.00 \\
\hline & & & & & & & & & & \\
\hline 1054 Average & 6.95 & 4.42 & 17.5 & 15.5 & 0.53 & 0.035 & 1841 & 3.96 & 32492 & 5.00 \\
\hline Std. Dev. & 0.43 & 0.51 & 1.5 & 1.4 & 0.02 & 0.005 & 26 & 0.06 & 507 & 0.00 \\
\hline CV\% & 6.2 & 11.6 & 8.5 & 8.7 & 4.0 & 15.4 & $\overline{1.4}$ & 1.5 & 1.6 & 0.1 \\
\hline
\end{tabular}

\section{Test Purpose:}

Collection of emissions data from LNG fueled vehicles for use in comparison with similar, diesel powered vehicles. 
Test Sequence Number: 1055

WVU Test Reference Number: RDC-1508-LNG-5MILES

Fleet Owner Full Name

Fleet Address

Fleet Address (City, State, Zip)

Vehicle Type

Vehicle ID Number (VIN)

Vehicle Manufacturer

Vehicle Model Year

Gross Vehicle Weight (GVW) (lb.)

Vehicle Total Curb Weight (Ib.)

Vehicle Tested Weight (lb.)

Odometer Reading (mile)

Transmission Type

Transmission Configuration

Number of Axles

Raley's Distribution Center

4061 Gateway Park Blvd.

Sacramento, CA 95834

Tractor Truck

1XKDD59X8VR745380

Kenworth

1997

80000

15680

42000

52700

Manual

10-Speed

3

Engine Type

Engine ID Number

Engine Displacement (Liter)

Cummins GL-10-300E

34849165

Number of Cylinders

10

Engine Rated Power (hp)

6

300

Primary Fuel

Test Cycle

LNG

Test Date

5 Mile Route

$2 / 25 / 98$

Engineer

Driver

W. Xie

B. Davis

Emissions Results (g/mile)

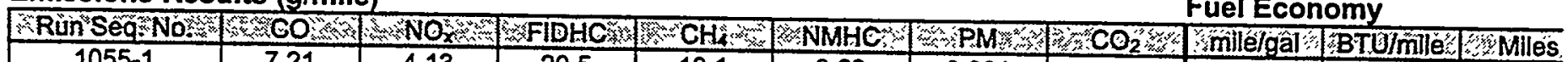

\begin{tabular}{|c|c|c|c|c|c|c|c|c|c|c|}
\hline $1055-1$ & 7.21 & 4.13 & 20.5 & 18.1 & 0.69 & 0.061 & 1793 & 4.04 & 31805 & 5.00 \\
\hline $1055-2$ & 7.20 & 3.32 & 20.7 & 18.3 & 0.69 & 0.058 & 1773 & 4.08 & 31475 & 5.01 \\
\hline $1055-3$ & 7.02 & 3.85 & 19.8 & 17.4 & 0.69 & 0.050 & 1768 & 4.10 & 31347 & 5.00 \\
\hline $1055-4$ & 6.97 & 4.54 & 18.5 & 16.2 & 0.70 & 0.059 & 1730 & 4.19 & 30642 & 5.01 \\
\hline $1055-5$ & 6.52 & 2.64 & 18.2 & 16.1 & 0.57 & 0.058 & 1764 & 4.12 & 31193 & 5.01 \\
\hline $1055-6$ & 6.48 & 2.90 & 19.2 & 16.9 & 0.66 & 0.056 & 1756 & 4.13 & 31097 & 5.00 \\
\hline & & & & & & & & & & \\
\hline 1055 Average & 6.90 & 3.56 & 19.5 & 17.2 & 0.67 & 0.057 & 1764 & 4.11 & 31260 & 5.00 \\
\hline Std. Dev. & 0.32 & 0.74 & 1.0 & 0.9 & 0.05 & 0.004 & 20 & 0.05 & 391 & 0.01 \\
\hline CV\% & 4.7 & 20.7 & 5.4 & 5.4 & 7.4 & 6.7 & 1.2 & 1.3 & 1.2 & 0.1 \\
\hline
\end{tabular}

Test Purpose: Collection of emissions data from LNG fueled vehicles for use in comparison with similar, diesel powered
vehicles.

\section{Observations:}

NOx emissions were very low compared to other similar LNG tractors, while the variation from test run to test run was high. 
Test Sequence Number: 1056

WVU Test Reference Number: RDC-1504-LNG-5MILES-R2

Fleet Owner Full Name

Fleet Address

Fleet Address (City, State, Zip)

Vehicle Type

Vehicle ID Number (VIN)

Vehicle Manufacturer

Vehicle Model Year

Gross Vehicle Weight (GWW) (lb.)

Vehicle Total Curb Weight (lb.)

Vehicle Tested Weight (lb.)

Odometer Reading (mile)

Transmission Type

Transmission Configuration

Number of Axles

Engine Type

Engine ID Number

Engine Displacement (Liter)

Number of Cylinders

Engine Rated Power (hp)

Primary Fuel

Test Cycle

Test Date

Engineer

Driver
Raley's Distribution Center

4061 Gateway Park Blvd.

Sacramento, CA 95834

Tractor Truck

1XKDD59X6VR745376

Kenworth

1997

80000

16540

42000

51600

Manual

10-Speed

3

Cummins GL-10-300E

34847352

10

6

300

LNG

5 Mile Route

$2 / 26 / 98$

W. Xie

B. Davis

Emissions Results (g/mile)

Fuel Economy

\begin{tabular}{|c|c|c|c|c|c|c|c|c|c|c|}
\hline Run SegNo & 500 & 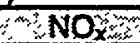 & FFIDHC & $\sqrt{\mathrm{CH}_{4}}$ & NMHE & किM & $\mathrm{San}$ & imilelgẩi & BTu/mile & ssomiless \\
\hline $1056-1$ & 8.45 & 4.27 & 50.1 & 45.1 & 0.79 & 0.055 & 1865 & 3.80 & 33786 & 4.99 \\
\hline $1056-2$ & 8.75 & 4.15 & 54.9 & 49.4 & 0.84 & 0.053 & 1801 & 3.91 & 32894 & 5.01 \\
\hline $1056-3$ & 8.39 & 5.24 & 33.4 & 29.9 & 0.72 & 0.051 & 1811 & 3.94 & 32581 & 5.00 \\
\hline $1056-4$ & 7.55 & 4.22 & 20.7 & 18.3 & 0.63 & 0.039 & 1811 & 4.00 & 32099 & 5.00 \\
\hline $1056-5$ & 8.19 & 5.06 & 37.2 & 33.3 & 0.74 & 0.048 & 1828 & 3.90 & 32946 & 5.00 \\
\hline 1056 Average & 897 & 459 & 303 & 550 & 071 & 0040 & 1902 & 009 & 32091 & 50 \\
\hline Std. Dev. & 0.45 & $\frac{7.05}{0.52}$ & $\frac{05.0}{13.6}$ & 124 & $\frac{0.14}{0.08}$ & $\frac{0.007}{0.007}$ & $\frac{1020}{25}$ & $\frac{0.91}{0.07}$ & $\frac{32001}{617}$ & $\frac{5.00}{0.01}$ \\
\hline CV\% & 5.4 & 11.3 & 34.7 & 35.2 & 10.3 & 13.3 & 1.4 & 1.9 & 1.9 & 0.1 \\
\hline
\end{tabular}

Test Purpose:

Re-test vehicle 1504 under sunny weather to determine the effect of rainy weather on emissions.

\section{Special Procedures:}

Cummins representative informed us that no tune up or any service would be done on this vehicle. So this vehicle was not re-tested during this trip any more.

\section{Observations:}

Driver complained that the vehicle was rough at 40MPH. All CO, CO2, NOx and PM emissions dropped comparing to those in test 1051 which was tested in rain, while $\mathrm{HC}$ was still out of analyzer range during $40 \mathrm{MPH}$ ramp. 
Test Sequence Number: 1057

WVU Test Reference Number: RDC-1505-LNG-5MILES

Fleet Owner Full Name

Fleet Address

Fleet Address (City, State, Zip)

Vehicle Type

Vehicle ID Number (VIN)

Vehicle Manufacturer

Vehicle Model Year

Gross Vehicle Weight (GWW) (lb.)

Vehicle Total Curb Weight (lb.)

Vehicle Tested Weight (lb.)

Odometer Reading (mile)

Transmission Type

Transmission Configuration

Number of Axles

\section{Engine Type}

Engine ID Number

Engine Displacement (Liter)

Number of Cylinders

Engine Rated Power (hp)

Primary Fuel

Test Cycle

Test Date

Engineer

Driver
Raley's Distribution Center

4061 Gateway Park Blvd.

Sacramento, CA 95834

Tractor Truck

1XKDD59X8VR745377

Kenworth

1997

80000

15720

42000

28800

Manual

10-Speed

3

Cummins GL-10-300E

34849168

10

6

300

LNG

5 Mile Route

$2 / 27 / 98$

W. Xie

B. Davis

Emissions Results (g/mile)

Fuel Economy

Run'Seg.No ;

\begin{tabular}{|c|c|c|c|c|c|c|c|c|c|c}
\hline $1057-1$ & 9.35 & 6.67 & 17.7 & 15.5 & 0.67 & 0.041 & 2036 & 3.58 & 35893 & 5.01 \\
\hline $1057-2$ & 10.12 & 7.77 & 18.0 & 15.8 & 0.68 & 0.037 & 2011 & 3.62 & 35507 & 5.00 \\
\hline $1057-3$ & 8.94 & 7.44 & 18.5 & 16.3 & 0.62 & 0.033 & 1959 & 3.71 & 34596 & 5.01 \\
\hline $1057-4$ & 8.70 & 6.55 & 17.4 & 15.2 & 0.68 & 0.036 & 2000 & 3.65 & 35245 & 5.00 \\
\hline $1057-5$ & 8.17 & 7.00 & 15.9 & $\mathrm{C}$ & $\mathrm{c}$ & 0.033 & 1929 & 3.79 & 33951 & 5.01 \\
\hline $1057-6$ & 8.42 & 7.06 & 17.2 & 15.1 & 0.67 & 0.039 & 1953 & 3.73 & 34419 & 5.01 \\
\hline & & & & & & & & & & \\
\hline 1057 Average & 8.95 & 7.08 & 17.5 & 15.6 & 0.67 & 0.037 & 1981 & 3.68 & 34935 & 5.01 \\
\hline Std. Dev. & 0.70 & 0.46 & 0.9 & 0.5 & 0.03 & 0.003 & 41 & 0.08 & 734 & 0.01 \\
\hline CV\% & 7.9 & 6.5 & 5.1 & 3.1 & 4.0 & 8.9 & 2.1 & 2.1 & 2.1 & 0.1 \\
\hline
\end{tabular}

\section{Test Purpose:}

Collection of emissions data from LNG fueled vehicles for use in comparison with similar, diesel powered vehicles.

\section{Special Procedures:}

Cummins representative informed us four days before the test that there was a regulator problem for the vehicle, which could not convert LNG to gas properly. This problem was fixed before the test. 
Test Sequence Number: 1058

WVU Test Reference Number: RDC-1501-LNG-5MILES-R

Fleet Owner Fuil Name

Fleet Address

Fleet Address (City, State, Zip)

Vehicle Type

Vehicle ID Number (VIN)

Vehicle Manufacturer

Vehicle Model Year

Gross Vehicle Weight (GWW) (lb.)

Vehicle Total Curb Weight (lb.)

Vehicle Tested Weight (Ib.)

Odometer Reading (mile)

Transmission Type

Transmission Configuration

Number of Axles

Engine Type

Engine ID Number

Engine Displacement (Liter)

Number of Cylinders

Engine Rated Power (hp)

Primary Fuel

Test Cycle

Test Date

Engineer

Driver
Raley's Distribution Center

4061 Gateway Park Blvd.

Sacramento, CA 95834

Tractor Truck

1XKDD59XOVR745373

Kenworth

1997

80000

16520

42000

38000

Manual

10-Speed

3

Cummins GL-10-300E

34845353

10

6

300

LNG

5 Mile Route

2/28/98

W. Xie

B. Davis

Emissions Results (g/mile)

Fuel Economy

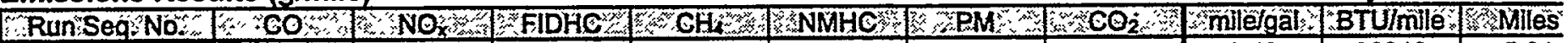

\begin{tabular}{|c|c|c|c|c|c|c|c|c|c|c}
\hline $1058-1$ & 9.74 & 4.30 & 19.7 & 17.4 & 0.63 & 0.033 & 2085 & 3.49 & 36840 & 5.01 \\
\hline $1058-2$ & 8.47 & 3.98 & 19.1 & 16.9 & 0.65 & 0.031 & 2036 & 3.58 & 35933 & 5.01 \\
\hline $1058-4$ & 10.31 & 4.81 & 20.4 & 18.0 & 0.66 & 0.034 & 1985 & 3.65 & 35164 & 5.01 \\
\hline $1058-5$ & 9.46 & 4.77 & 19.7 & 17.3 & 0.66 & 0.034 & 1962 & 3.70 & 34723 & 5.01 \\
\hline $1058-6$ & 9.11 & 3.94 & 19.2 & 16.9 & 0.69 & 0.034 & 2025 & 3.59 & 35767 & 5.01 \\
\hline & & & & & & & & & & \\
\hline 1058 Average & 9.42 & 4.36 & 19.6 & 17.3 & 0.66 & 0.033 & 2019 & 3.60 & 35686 & 5.01 \\
\hline Std. Dev. & 0.69 & 0.42 & 0.5 & 0.5 & 0.02 & 0.001 & 48 & 0.08 & 806 & 0.00 \\
\hline CV\% & 7.3 & 9.6 & 2.5 & 2.7 & 3.1 & 4.0 & 2.4 & 2.2 & 2.3 & 0.1 \\
\hline
\end{tabular}

\section{Test Purpose:}

Collection of emissions data from LNG fueled vehicle after it has been serviced to determine the effect, if any, on emissions.

\section{Special Procedures:}

One of the spark plugs was found bad and replaced after the previous test (see test number 1047). Vehicle then ran normally.

\section{Observations:}

$\mathrm{HC}$ and $\mathrm{PM}$ emissions dropped dramatically, while $\mathrm{CO}$ dropped slightly and $\mathrm{CO} 2$ stayed about the same comparing to previous test (see test number 1047). 
Test Sequence Number: 1059

WVU Test Reference Number: RDC-1586-D2-5MILES

Fleet Owner Full Name

Fieet Address

Fleet Address (City, State, Zip)

Vehicle Type

Vehicle ID Number (VIN)

Vehicle Manufacturer

Vehicle Model Year

Gross Vehicle Weight (GVW) (Ib.)

Vehicle Total Curb Weight (lb.)

Vehicle Tested Weight (lb.)

Odometer Reading (mile)

Transmission Type

Transmission Configuration

Number of Axles

Engine Type

Engine ID Number

Engine Displacement (Liter)

Number of Cylinders

Engine Rated Power (hp)

Primary Fuel

Test Cycle

Test Date

Engineer

Driver
Raley's Distribution Center 4061 Gateway Park Blvd.

Sacramento, CA 95834

Tractor Truck

1XKBD99X9TS690099

Kenworth

1995

80000

14440

42000

205700

Manual

10-Speed

3

Cummins M11-330E

34776206

10.8

6

330

D2

5 Mile Route

$3 / 2 / 98$

W. Xie

B. Davis

Emissions Results (g/mile)

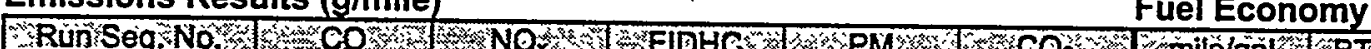

\begin{tabular}{|c|c|c|c|c|c|c|c|c|}
\hline numiseg.in & $C_{0}$ & $\mathrm{NO}_{\mathrm{x}}$ & 1 & 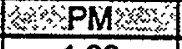 & $\mathrm{CO}_{2}$ & somilèlgàl & a Bufmile & Miles \\
\hline 1059-2 & 2.27 & 21.1 & 1.97 & 1.92 & 1689 & 5.99 & 21701 & 5.00 \\
\hline $1059-3$ & 2.22 & 23.2 & 1.89 & 1.69 & 1646 & 6.15 & 21154 & 5.00 \\
\hline $1059-4$ & 2.50 & 20.5 & 1.94 & 1.72 & 1631 & 6.20 & 20961 & 5.01 \\
\hline 1059-5 & 2.27 & 17.8 & 1.93 & 1.62 & 1643 & 6.16 & 21116 & 5.01 \\
\hline $1059-6$ & 2.32 & 21.4 & 2.03 & 1.90 & 1663 & 6.08 & 21376 & 5.01 \\
\hline & & & & & & & & \\
\hline 1059 Average & 2.32 & 20.8 & 1.95 & 1.77 & 1654 & 6.12 & 21262 & 5.01 \\
\hline Std. Dev. & 0.11 & 1.9 & 0.05 & 0.13 & 22 & 0.08 & 287 & 0.01 \\
\hline CV\% & 4.7 & 9.4 & 2.8 & 7.3 & 1.4 & 1.3 & 1.3 & 0.1 \\
\hline
\end{tabular}

\section{Test Purpose:}

Collection of emissions data from diesel fueled vehicles for use in comparison with similar, LNG powered vehicles. 
Test Sequence Number: 1060

WVU Test Reference Number: RDC-1592-D2-5MILES

Fleet Owner Full Name

Fleet Address

Fleet Address (City, State, Zip)

Vehicle Type

Vehicle ID Number (VIN)

Vehicle Manufacturer

Vehicle Model Year

Gross Vehicle Weight (GWW) (lb.)

Vehicle Total Curb Weight (lb.)

Vehicle Tested Weight (lb.)

Odometer Reading (mile)

Transmission Type

Transmission Configuration

Number of Axles

Engine Type

Engine ID Number

Engine Displacement (Liter)

Number of Cylinders

Engine Rated Power (hp)

Primary Fuel

Test Cycle

Test Date

Engineer

Driver
Raley's Distribution Center

4061 Gateway Park Blvd.

Sacramento, CA 95834

Tractor Truck

1XKBD99X2TS712783

Kenworth

1995

80000

14960

42000

174000

Manual

10-Speed

3

Cummins M11-330E

34786945

10.8

6

330

D2

5 Mile Route

$3 / 3 / 98$

W. Xie

B. Davis

Emissions Results (g/mile)

Fuel Economy

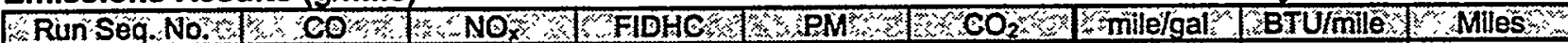

\begin{tabular}{|c|c|c|c|c|c|c|c|c|}
\hline $1060-1$ & 2.11 & 23.7 & 1.64 & 1.19 & 1684 & 6.01 & 21620 & 4.99 \\
\hline $1060-2$ & 2.21 & 22.1 & 1.63 & 1.17 & 1619 & 6.25 & 20792 & 5.00 \\
\hline $1060-3$ & 2.07 & 18.8 & 1.66 & 1.16 & 1608 & 6.29 & 20654 & 5.00 \\
\hline $1060-4$ & 2.05 & 21.3 & 1.67 & 1.16 & 1624 & 6.23 & 20852 & 5.00 \\
\hline $1060-5$ & 2.35 & 18.4 & 1.63 & 1.16 & 1610 & 6.29 & 20677 & 5.00 \\
\hline & & & & & & & & \\
\hline 1060 Average & 2.16 & 20.9 & 1.65 & 1.17 & 1629 & 6.22 & 20919 & 5.00 \\
\hline Std. Dev. & 0.12 & 2.3 & 0.02 & 0.01 & 31 & 0.12 & 400 & 0.00 \\
\hline CV\% & 5.8 & 10.8 & 1.1 & 1.0 & 1.9 & 1.9 & 1.9 & 0.1 \\
\hline
\end{tabular}

\section{Test Purpose:}

Collection of emissions data from diesel fueled vehicles for use in comparison with similar, LNG powered vehicles. 
Test Sequence Number: 1061

WVU Test Reference Number: RDC-1593-D2-5MILES

Fleet Owner Full Name

Fleet Address

Fleet Address (City, State, Zip)

Vehicle Type

Vehicle ID Number (VIN)

Vehicle Manufacturer

Vehicle Model Year

Gross Vehicle Weight (GWW) (Ib.)

Vehicle Total Curb Weight (lb.)

Vehicle Tested Weight (lb.)

Odometer Reading (mile)

Transmission Type

Transmission Configuration

Number of Axles

Engine Type

Engine ID Number

Engine Displacement (Liter)

Number of Cylinders

Engine Rated Power (hp)

Primary Fuel

Test Cycle

Test Date

Engineer

Driver
Raley's Distribution Center

4061 Gateway Park Blvd.

Sacramento, CA 95834

Tractor Truck

1XKBD99X4TS712784

Kenworth

1995

80000

14960

42000

209200

Manual

10-Speed

3

Cummins M11-330E

34787997

10.8

6

330

D2

5 Mile Route

$3 / 4 / 98$

W. Xie

B. Davis

Emissions Results (g/mile)

Fuel Economy

Fun Sëq No.

\begin{tabular}{|c|c|c|c|c|c|c|c|c|}
\hline $1061-3$ & 2.15 & 18.6 & 1.46 & 0.74 & 1668 & 6.07 & 21412 & 5.02 \\
\hline $1061-4$ & 2.55 & 15.7 & 1.51 & 0.72 & 1702 & 5.95 & 21850 & 5.00 \\
\hline $1061-5$ & 2.14 & 18.7 & 1.48 & 0.70 & 1725 & 5.87 & 22138 & 5.01 \\
\hline $1061-6$ & 2.24 & 16.3 & 1.55 & 0.70 & 1704 & 5.94 & 21883 & 5.01 \\
\hline $1061-7$ & 2.41 & 16.4 & 1.50 & 0.73 & 1659 & 6.10 & 21305 & 5.01 \\
\hline & & & & & & & & \\
\hline 1061 Average & 2.30 & 17.1 & 1.50 & 0.72 & 1692 & 5.99 & 21718 & 5.01 \\
\hline Std. Dev. & 0.18 & 1.4 & 0.03 & 0.02 & 27 & 0.10 & 348 & 0.00 \\
\hline CV\% & 7.7 & 8.2 & 2.2 & 2.6 & 1.6 & 1.6 & 1.6 & 0.1 \\
\hline
\end{tabular}

\section{Test Purpose:}

Collection of emissions data from diesel fueled vehicles for use in comparison with similar, LNG powered vehicles. 
Table 1: Comparison of Emissions from Sacramento LNG and Diesel Fueled Tractors

Emission Summary for LNG Powered Tractors in Sacramento, CA

\begin{tabular}{|c|c|c|c|c|c|c|c|c|c|c|c|c|c|}
\hline STESTIDE & 样 & Oucte Is & Trost & aMile & Was & tat & 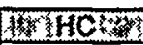 & 7 & WC & 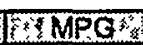 & 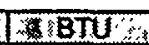 & CH4 & DNMHO \\
\hline 1058 & RDC-1501-LNG-5MILES-R & 5 Mile Route & $2 / 28 / 98$ & 38000 & 9.42 & 4.36 & 19.63 & 0.033 & 2019 & 3.60 & 35686 & 17.31 & 0.66 \\
\hline 1048 & RDC-1502-LNG-5MILES & 5 Mile Route & $2 / 20 / 98$ & 38600 & 8.44 & 4.65 & 25.49 & 0.041 & 1816 & 3.96 & 32469 & 22.69 & 0.63 \\
\hline 1052 & RDC-1503-LNG-5MLES & 5 Mile Route & $2 / 24 / 98$ & 46000 & 7.95 & 7.93 & 16.14 & 0.047 & 1633 & 4.45 & 28891 & 14.36 & 0.60 \\
\hline 1056 & RDC-1504-LNG-5MILES-R2 & 5 Mile Roule & $2 / 26 / 98$ & 51600 & 8.27 & 4.59 & 39.27 & 0.049 & 1823 & 3.91 & 32861 & 35.20 & 0.74 \\
\hline 1057 & RDC-1505-LNG-5MILES & R Route & $2 / 27 / 98$ & 28800 & 8.95 & 7.08 & 17.47 & 0.037 & 1981 & 3.68 & 34935 & 15.60 & 0.67 \\
\hline 1054 & RDC-1506-LNG-5MILES & 5 Mile Route & $2 / 25 / 98$ & 44700 & 6.95 & 4.42 & 17.54 & 0.035 & 1841 & 3.96 & 32492 & 15.53 & 0.53 \\
\hline 1053 & RDC-1507-LNG-5MILES & 5 Mile Route & $2 / 24 / 98$ & 46000 & 6.51 & 5.42 & 15.97 & 0.025 & 1688 & 4.32 & 29784 & 14.12 & 0.50 \\
\hline 1055 & RDC-1508-LNG-5MILES & 5 Mile Route & $2 / 25 / 98$ & 52700 & 6.90 & 3.56 & 19.48 & 0.057 & 1764 & 4.11 & 31260 & 17.16 & 0.67 \\
\hline & & & Average: & 43300 & 7.92 & 5.25 & 21.37 & 0.040 & 1821 & 4.00 & 32297 & 19.00 & 0.62 \\
\hline
\end{tabular}

Emission Summary for Diesel Powered Tractors in Sacramento, CA

\begin{tabular}{|c|c|c|c|c|c|c|c|c|c|c|c|}
\hline Mes & $\sqrt{32}$ & SECiciolo & 30 & 瀷 & 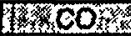 & W' & 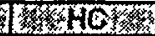 & W & 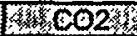 & MPQ & 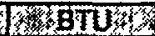 \\
\hline 1059 & RDC-1586-D2-5MILES & 5 Mile Route & $3 / 2 / 98$ & 205700 & 2.32 & 20.79 & 1.95 & 1.77 & 1654 & 6.12 & 21262 \\
\hline 1060 & RDC-1592-D2-5MILES & 5 Mile Route & $3 / 3 / 98$ & 174000 & 2.16 & 20.85 & 1.65 & 1.17 & 1629 & 6.22 & 20919 \\
\hline 1061 & RDC-1593-D2-5MILES & 5 Mile Route & $3 / 4 / 98$ & 209200 & 2.30 & 17.14 & 1.50 & 0.72 & 1692 & 5.99 & 21718 \\
\hline & & & Average: & 196300 & 2.26 & 19.59 & 1.70 & 1.22 & 1658 & 6.11 & 21299 \\
\hline
\end{tabular}

